\title{
First Responders Guide to Computer Forensics: Advanced Topics
}

\author{
Richard Nolan \\ Marie Baker \\ Jake Branson \\ Josh Hammerstein \\ Kris Rush \\ Cal Waits \\ Elizabeth Schweinsberg
}

September 2005

HANDBOOK

CMU/SEI-2005-HB-003 



\title{
First Responders Guide to Computer Forensics: Advanced Topics
}

CMU/SEI-2005-HB-003

\author{
Richard Nolan \\ Marie Baker \\ Jake Branson \\ Josh Hammerstein \\ Kris Rush \\ Cal Waits \\ Elizabeth Schweinsberg
}

September 2005

CERT Training and Education 
This report was prepared for the

SEI Administrative Agent

$\mathrm{ESC} / \mathrm{XPK}$

5 Eglin Street

Hanscom AFB, MA 01731-2100

The ideas and findings in this report should not be construed as an official DoD position. It is published in the interest of scientific and technical information exchange.

FOR THE COMMANDER

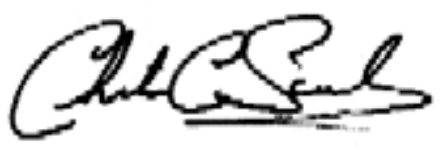

Christos Scondras

Chief of Programs, XPK

This work is sponsored by the SEI FFRDC primary sponsor and the Department of Homeland Security. The Software Engineering Institute is a federally funded research and development center sponsored by the U.S. Department of Defense.

Copyright 2005 Carnegie Mellon University.

\section{NO WARRANTY}

THIS CARNEGIE MELLON UNIVERSITY AND SOFTWARE ENGINEERING INSTITUTE MATERIAL IS FURNISHED ON AN "AS-IS" BASIS. CARNEGIE MELLON UNIVERSITY MAKES NO WARRANTIES OF ANY KIND, EITHER EXPRESSED OR IMPLIED, AS TO ANY MATTER INCLUDING, BUT NOT LIMITED TO, WARRANTY OF FITNESS FOR PURPOSE OR MERCHANTABILITY, EXCLUSIVITY, OR RESULTS OBTAINED FROM USE OF THE MATERIAL. CARNEGIE MELLON UNIVERSITY DOES NOT MAKE ANY WARRANTY OF ANY KIND WITH RESPECT TO FREEDOM FROM PATENT, TRADEMARK, OR COPYRIGHT INFRINGEMENT.

Use of any trademarks in this report is not intended in any way to infringe on the rights of the trademark holder.

Internal use. Permission to reproduce this document and to prepare derivative works from this document for internal use is granted, provided the copyright and "No Warranty" statements are included with all reproductions and derivative works.

External use. Requests for permission to reproduce this document or prepare derivative works of this document for external and commercial use should be addressed to the SEI Licensing Agent.

This work was created in the performance of Federal Government Contract Number FA8721-05-C-0003 with Carnegie Mellon University for the operation of the Software Engineering Institute, a federally funded research and development center. The Government of the United States has a royalty-free government-purpose license to use, duplicate, or disclose the work, in whole or in part and in any manner, and to have or permit others to do so, for government purposes pursuant to the copyright license under the clause at 252.227-7013.

For information about purchasing paper copies of SEI reports, please visit the publications portion of our Web site (http://www.sei.cmu.edu/publications/pubweb.html). 


\section{Table of Contents}

Executive Summary ................................................................................. xi

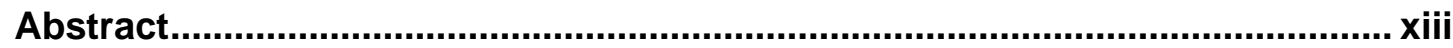

1 Module 1: Log File Analysis................................................................. 1

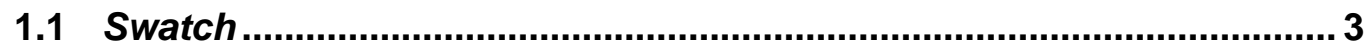

1.1.1 Swatch Log Monitor.............................................................. 4

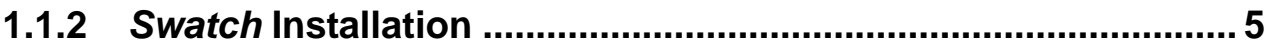

1.1.2.1 Installing Perl Modules................................................ 5

1.1.2.2 Installing Swatch ............................................... 6

1.1.3 Swatch Configuration .............................................................. 8

1.1.3.1 The Configuration File Location .................................. 9

1.1.3.2 Adding Rules to the Configuration File ........................ 9

1.1 .4 Swatch Execution ................................................................ 15

1.1 .5 Summary............................................................................ 17

1.2 Microsoft Log Parser 2.2 ................................................................... 18

1.2.1 Microsoft Log Parser Features ............................................. 20

1.2.2 Log Parser Installation ........................................................... 21

1.2.3 Log Parser Input and Output .............................................. 22

1.2.3.1 Input Formats........................................................... 22

1.2.3.2 Output Formats ............................................... 24

1.2.4 Log Parser Queries............................................................... 26

1.2.4.1 Query Examples ..................................................... 27

1.2.5 Log Parser COM Objects......................................................... 29

1.2.5.1 Creating Custom Input Formats................................... 29

1.2.5.2 Using the Log Parser COM API ................................ 30

1.2.6 Log Parser Execution ......................................................... 31

1.2 .7 Summary........................................................................... 33

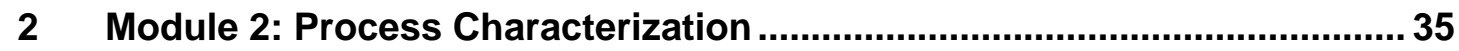

2.1 Understanding a Running Process ................................................. 36

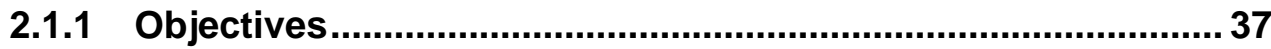

2.1.2 Programs, Processes, and Threads.................................... 38

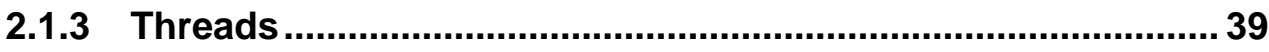

2.1.3.1 Displaying Threads for a Running Process.................. 39 
2.1.3.2 Sysinternals Process Explorer ................................ 40

2.1.4 Process Tree Structure ........................................................ 43

2.1.4.1 pstree (Linux)................................................... 44

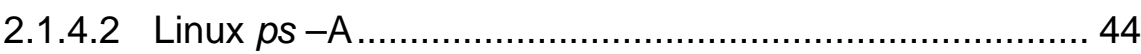

2.1.5 Process Descriptions ............................................................. 46

2.1.6 Process Hashes (National Software Reference Library)........ 47

2.1.7 Process Analysis Checklist ................................................. 49

2.1.8 Common Process Characteristics ........................................ 51

2.1.8.1 Process Filenames ................................................ 51

2.1.8.2 Open Ports ......................................................... 53

2.1.8.3 Open Files ........................................................... 55

2.1.8.4 Base Priority .......................................................... 56

2.1.8.5 Process Times and Terminated Processes.................... 58

2.1.8.6 Location of Process Image ................................... 60

2.1.8.7 Survivable Processes ................................................. 61

2.1.8.8 Process Forensic Tasks............................................. 66

2.2 Automated Process Collection ......................................................... 76

2.2.1 Objectives............................................................................ 77

2.2.2 First Responder Utility (FRU) ................................................. 78

2.2.2.1 First Responder Utility (FRUC) Setup ....................... 79

2.2.3 Forensic Server Project (FSP) .......................................... 82

2.2.3.1 FSP Setup ....................................................... 82

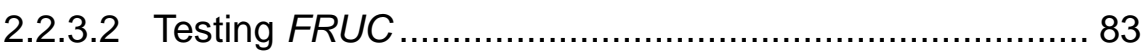

2.2.3.3 Output of FRUC .................................................. 84

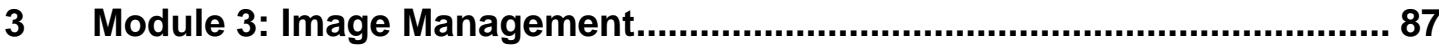

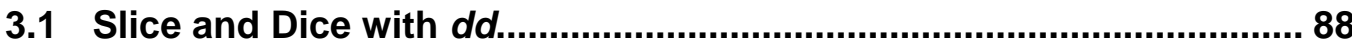

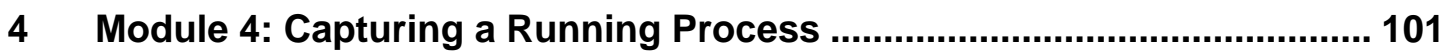

4.1.1 Hedons and Dolors............................................................. 103

4.1.2 Capturing a Process on a Windows System......................... 104

$5 \quad$ Module 5: Understanding Spoofed Email...............................................113

5.1 Objectives .........................................................................................114

5.2 Identifying Spoofed Email ...................................................................115

5.2.1 Definition of the Problem ...................................................116

5.2.2 Understanding the Process of Sending and Receiving Email ..........................................................................................117

5.2.2.1 The Life Cycle of an Email.....................................117

5.2.2.2 Overview of the Simple Mail Transfer Protocol.............119

5.2.3 Understanding Email Headers.............................................. 123

5.2.3.1 Interpreting Email Headers...................................... 123

5.2.4 How Spoofed Email Is Sent ................................................. 127 
5.2.4.1 Open Mail Relay .............................................. 127

5.2.4.2 Compromised Machines ........................................... 129

5.2.4.3 Self-Owned Mail Servers .......................................... 129

5.2.4.4 Temporary Accounts ............................................ 129

5.2.4.5 Hijacked Accounts ............................................... 129

5.2.5 How to Identify Spoofed Email ............................................ 130

5.2.5.1 Carefully Examine the "Received" Headers................ 130

5.2.5.2 Look Out for Spoofed Headers ................................. 132

5.2.5.3 Comparing Timestamps ...................................... 133

5.3 Tracing the Origins of a Spoofed Email.......................................... 135

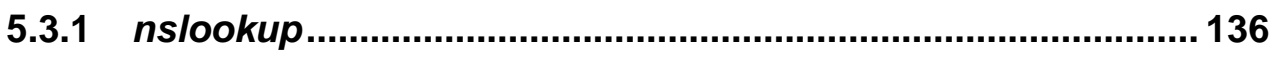

5.3.2 whois ......................................................................................... 139

5.3.2.1 IP Block Identification............................................. 139

5.3.2.2 WHOIS Information for a Domain Name ................... 142

5.3.3 Traceroute .......................................................................... 144

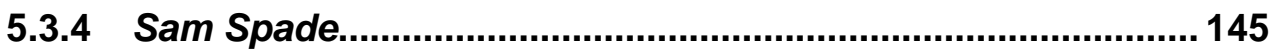

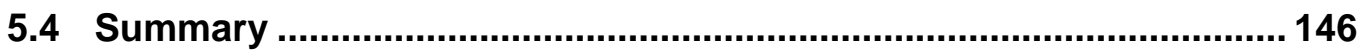

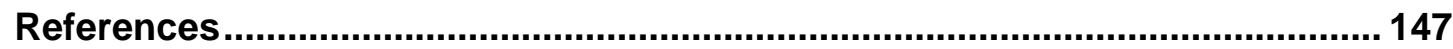




\section{List of Figures}

Figure 1: Example Run of the Swatch Configuration File ............................... 14

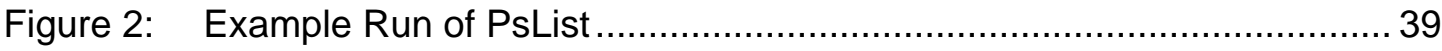

Figure 3: $\quad$ Sysinternals Process Explorer Utility.......................................... 40

Figure 4: Verifying a Process Image in Process Explorer .............................. 41

Figure 5: $\quad$ The Strings Tab in Process Explorer ............................................ 41

Figure 6: Displaying a Process Tree Using PsList...................................... 43

Figure 7: $\quad$ Displaying a Process Tree Using pstree ...................................... 44

Figure 8: Displaying PID Assignments Using ps ............................................. 45

Figure 9: $\quad$ WinTasks Process Description ................................................ 47

Figure 10: Listing Process Filenames Using pulist..................................... 52

Figure 11: Displaying Open Ports Using fport ............................................ 53

Figure 12: Displaying Open Ports Using netstat...................................... 54

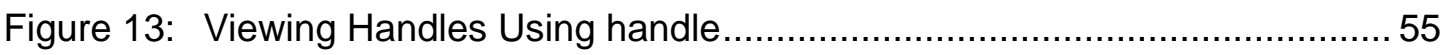

Figure 14: Displaying Which Process Has Port 6002 Open ............................. 56

Figure 15: Displaying Who Has the Bash Shell Open ...................................... 56

Figure 16: Displaying All the Currently Open Files by the User Root ..................... 56

Figure 17: Listing Priority Levels Using pslist .......................................... 57

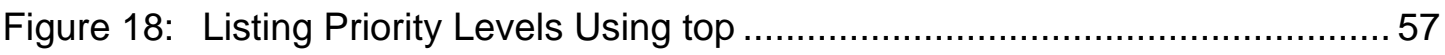

Figure 19: Displaying the Priority Level for a Specific Process ............................ 57 


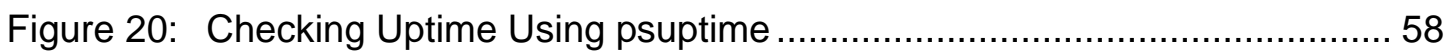

Figure 21: Checking Elapsed Time for a Process Using pslist .......................... 58

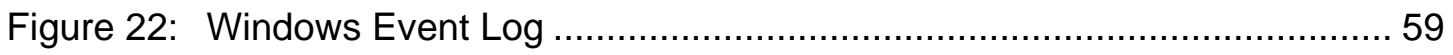

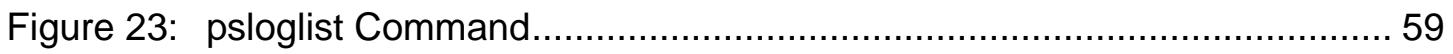

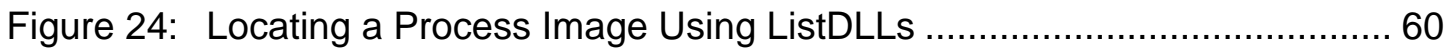

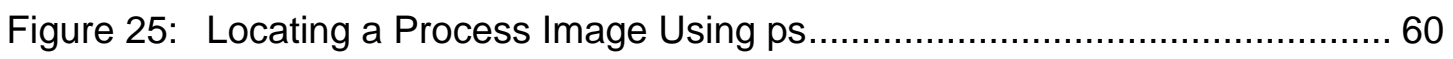

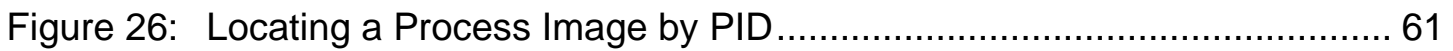

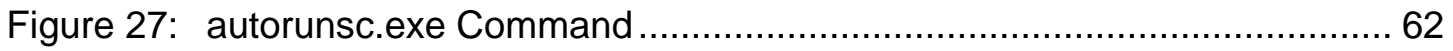

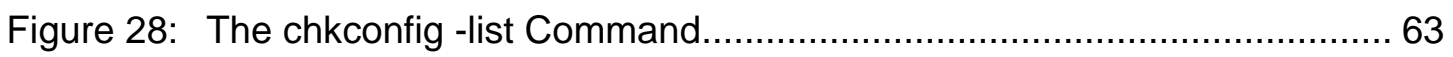

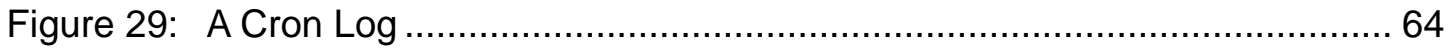

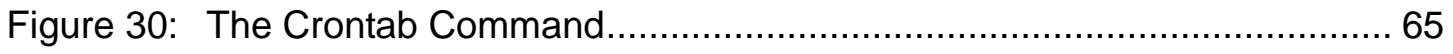

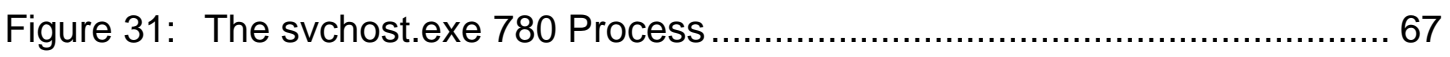

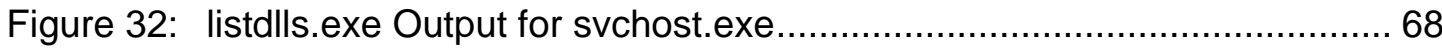

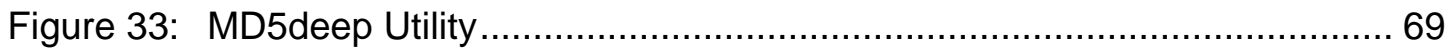

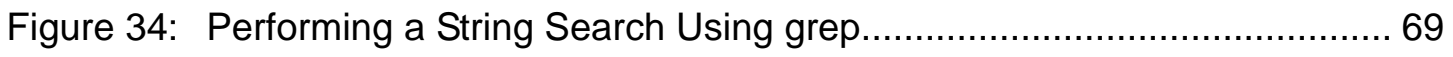

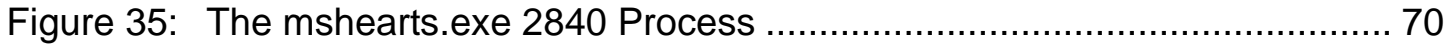

Figure 36: listdlls.exe Output for the mshearts Process .................................. 71

Figure 37: MD5deep.exe Command Line Arguments .................................... 71

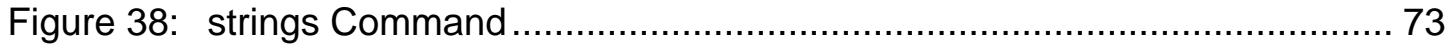

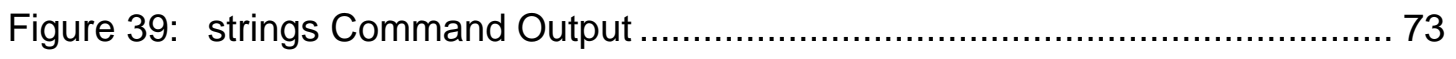

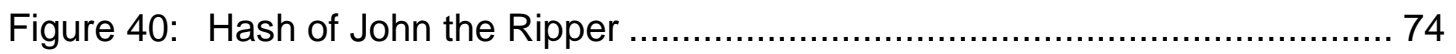

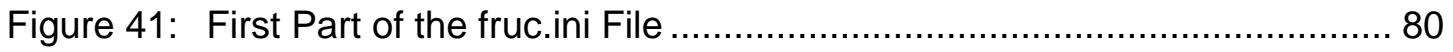

Figure 42: Second Part of the fruc.ini File .............................................. 80 
Figure 43: Final Part of fruc.ini File.

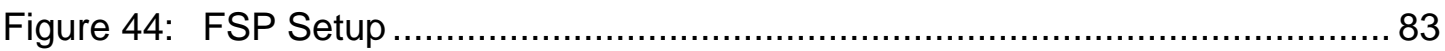

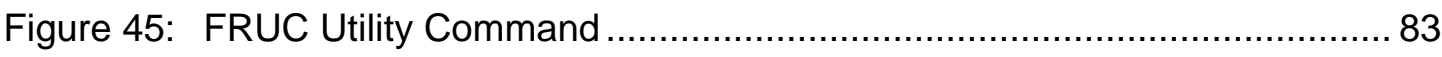

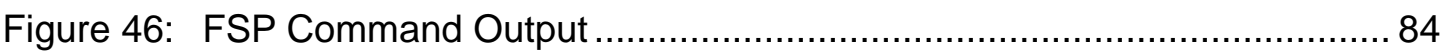

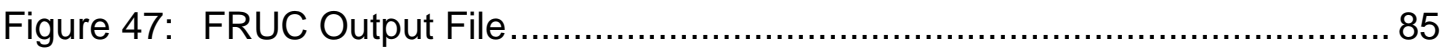

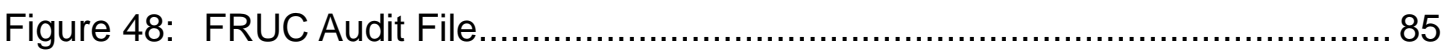

Figure 49: Result of Using md5 to Calculate a Hash Value.................................. 92

Figure 50: Confirming the Result of Splitting Images ....................................... 92

Figure 51: Result of Using cat and md5sum to Check the Integrity of Split

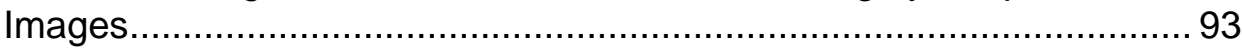

Figure 52: Result of Using md5sum to Check the Integrity of a New Image ........... 94

Figure 53: Finding a .jpg Tag in a Captured Image ............................................ 96

Figure 54: Decimal Form of the Beginning of the .jpg File ............................... 96

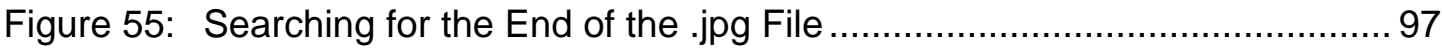

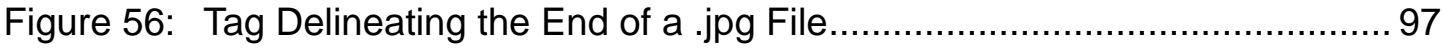

Figure 57: Decimal Address for the End of the .jpg File ............................... 97

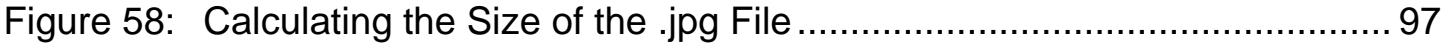

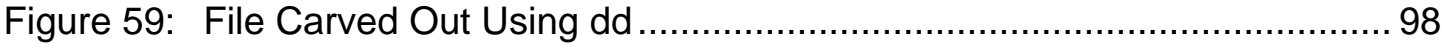

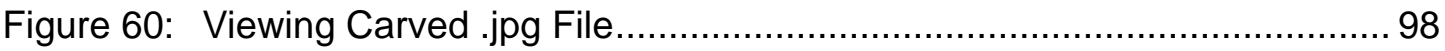

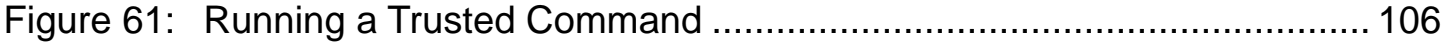

Figure 62: Command Shell Spawned from a Trusted CD.............................. 106

Figure 63: netcat Command to Listen on Port 3333 ......................................... 106

Figure 64: Using Trusted pslist and netcat to Specify IP Address and Listening Port. 
Figure 65: Looking for Suspicious Processes Using cat

Figure 66: Suspicious Process Found................................................. 107

Figure 67: netcat Command to Listen on Port 4444..................................... 108

Figure 68: Specifying netcat Listener Machine and Port ................................ 108

Figure 69: Viewing Path to a Suspicious Process ........................................... 108

Figure 70: Setting Up a Listening Session on a Suspicious Process .................. 109

Figure 71: Collecting the Executable of a Suspicious Process ........................... 109

Figure 72: Calculating a Hash of a Captured Process ...................................... 109

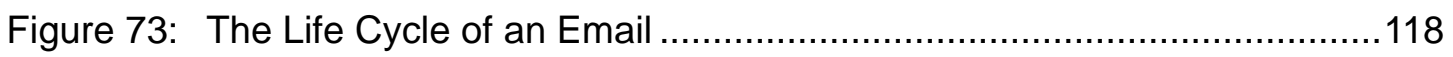

Figure 74: Mail Delivery for Valid Users .................................................... 128

Figure 75: Spoofed Email via an Open Relay .......................................... 128

Figure 76: nslookup of Valid Fully Qualified Domain Name ............................. 137

Figure 77: nslookup of Falsified Host Information ......................................... 138

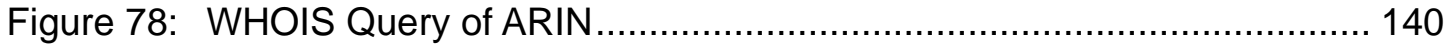

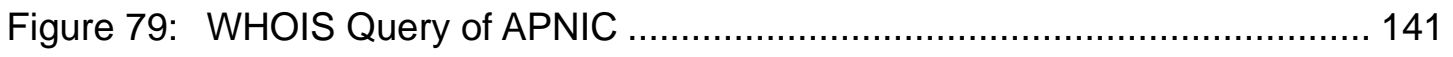

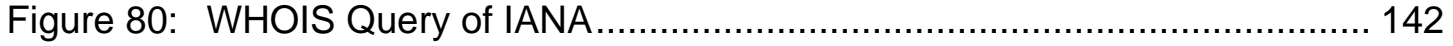

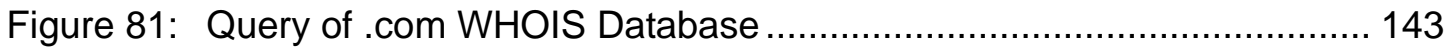

Figure 82: Query of the Registrar's WHOIS Database ................................... 143

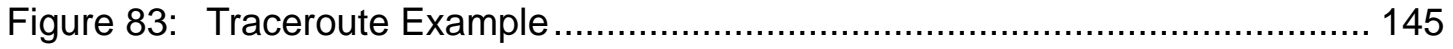




\section{List of Tables}

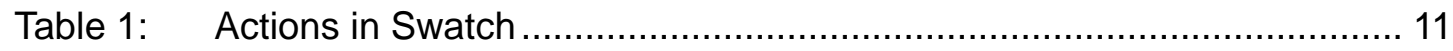

Table 2: $\quad$ time_regex for Popular Services ................................................. 13

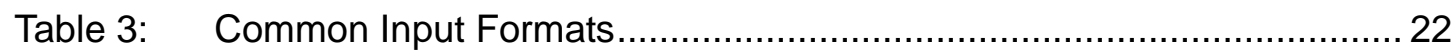

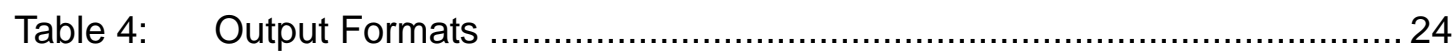

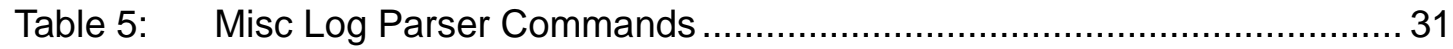

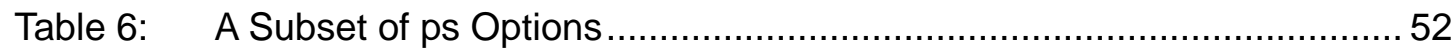

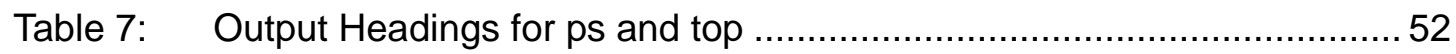

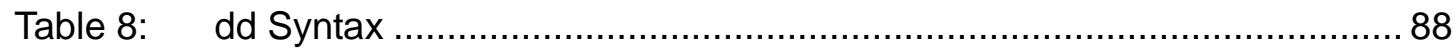

Table 9: $\quad$ Tools for Capturing Running Processes ....................................... 104

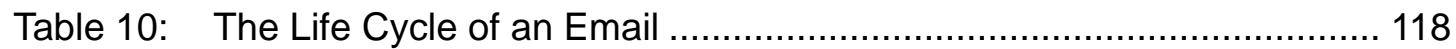

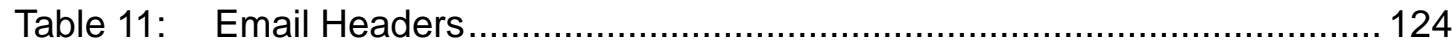




\section{Executive Summary}

First Responders Guide to Computer Forensics: Advanced Topics expands on the technical material presented in SEI handbook CMU/SEI-2005-HB-001, First Responders Guide to Computer Forensics [Nolan 05]. While the latter presented techniques for forensically sound collection of data and reviewed the fundamentals of admissibility pertaining to electronic files, this handbook focuses exclusively on more advanced technical operations like process characterization and spoofed email. It is designed for experienced security and network professionals who already have a fundamental understanding of forensic methodology. Therefore, emphasis is placed on technical procedures and not forensic methodology.

The first module focuses on log file analysis as well as exploring techniques for using common analysis tools such as Swatch and Log Parser. The second module focuses on advanced techniques for process characterization, analysis, and volatile data recovery. The third module demonstrates advanced usage of the $d d$ command-line utility. Topics include how to slice an image and reassemble it with $d d$, carving out a section of data with $d d$, and imaging a running process with $d d$. The fourth and final module examines spoofed email messages. This module looks at the RFCs for email, describes how email messages are spoofed, and presents some techniques for identifying and tracing spoofed email.

Our focus is to provide system and network administrators with advanced methodologies, tools, and procedures for applying sound computer forensics best practices when performing routine log file reviews, network alert verifications, and other routine interactions with systems and networks. The final goal is to create trained system and network professionals who are able to understand the fundamentals of computer forensics so that in the normal course of their duties they can safely preserve technical information related to network alerts and other security issues. This handbook is not intended to be a training guide for computer forensics practitioners, but rather an advanced resource for system and network security professionals who are charged with performing first responder functions. The target audience includes system and network administrators, law enforcement, and any information security practitioners who find themselves in the role of first responders. The handbook should help the target audience to

- install, configure, and use Swatch to analyze log files

- install, configure, and use Log Parser to analyze log files

- understand advanced elements of a running process

- perform an automated collection of volatile data

- carve out data using the $d d$ command-line utility

- use the $d d$ command-line utility to slice and reassemble images and files 
- understand spoofed email

- identify reliable information in an email header 


\section{Abstract}

This handbook expands on the technical material presented in SEI handbook CMU/SEI2005-HB-001, First Responders Guide to Computer Forensics. While the latter presented techniques for forensically sound collection of data and explained the fundamentals of admissibility pertaining to electronic files, this handbook covers more advanced technical operations such as process characterization and spoofed email. It describes advanced methodologies, tools, and procedures for applying computer forensics when performing routine log file reviews, network alert verifications, and other routine interactions with systems and networks. The material will help system and network professionals to safely preserve technical information related to network alerts and other security issues. 


\title{
1 Module 1: Log File Analysis
}

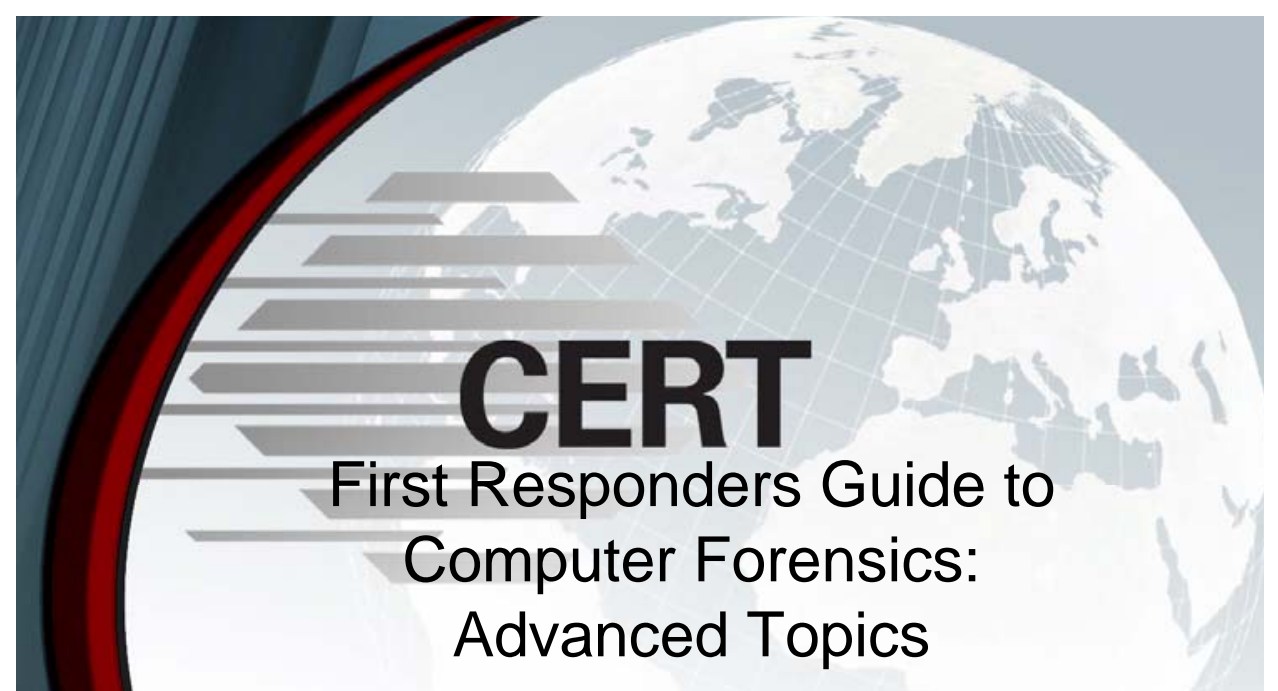

\author{
Module 1: \\ Log File Analysis
}

(c) 2005 Carnegie Mellon University

Software Engineering Institute

This module focuses on log file analysis, specifically post-event analysis using Swatch and Log Parser. We explain how to install, configure, and execute Swatch and Log Parser and provide several sample configurations for each. 


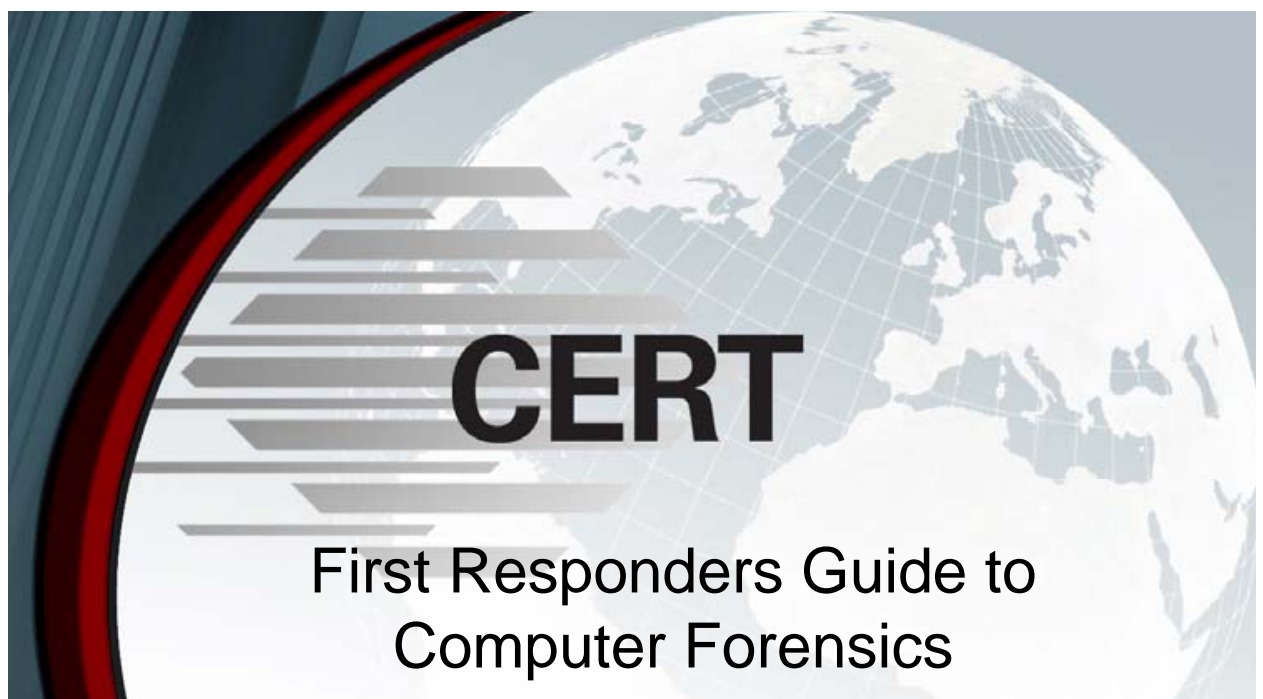

Swatch 


\section{Overview}

Why Swatch?

Installation

Configuration

Execution

Forensics, in this case, is the act of looking for events after they have happened, not performing an investigation for law enforcement.

\subsection{Swatch}

The focus is on using Swatch and Log Parser as forensic analysis tools, meaning that they will be used on logs after an incident has occurred. If you are planning to use these techniques on files involved in a law enforcement investigation, please make sure you prepare the files according to established best practices before use. 


\section{Swatch Log Monitor}

Swatch, the Simple Watcher, is an open

source log monitoring tool written in Perl for

use primarily on UNIX/Linux systems.

Swatch can be used to monitor current logs on running servers, or to examine older logs.

The configuration file contains a list of regular expressions to look for and actions to take, if any are found, called rules.

\section{While originally designed for use with syslog files, Swatch can be used on any file.}

\subsubsection{Swatch Log Monitor}

Log files are useful only if they are read. After an incident, log files often have clues as to what happened. However, many servers produce large volumes of log information, often spread out over more than one file, so sifting through this data can be tedious and time consuming. As an added problem, different servers have different log formats. If it is necessary to compare files, it can be challenging to match up fields.

Swatch, the Simple Watcher log monitoring tool, is capable of searching a file for a list of strings and then performing specific actions when one is found. It was designed to do realtime monitoring of server log files but can also be set to process a stand-alone file. Swatch was designed to work with syslog files, but it can be used on any file.

Swatch was written in Perl, and because of the way it is installed it is best used on a Linux system. It is an open source tool, and the project is maintained on SourceForge.

Throughout this module we will consider more heavily the case where Swatch is used to examine older log files as opposed to active log files. 


\section{Swatch Installation}

Requirements: Perl 5, make utility, tar utility

Download Swatch from the SourceForge project:

http://sourceforge.net/projects/swatch/

Download the throttle patch from http://www.cert.org

Obtain and install the additional Perl modules:

- Time::HiRes

- Date::Calc

- Date::Format

- File::Tail

Make Swatch - On the command line, type in progression:

tar zxvf swatch-3.1.1.tar.gz

patch $-\mathrm{p} 0<$ throttle.patch

cd swatch-3.1.1

perl Makefile.PL

make

make test

make install

\subsubsection{Swatch Installation}

Swatch has the same installation process as a Perl module. You download a tarball, uncompress it, expand it, and build it. The tool installs itself in /usr/bin, and you can use it from any directory. It also installs a manual page.

To begin, make sure that Perl 5 is installed on the machine. Later versions of Perl may come with some of the necessary modules installed already. You also need the ability to use the GNU utility make to fully install Swatch.

\subsubsection{Installing Perl Modules}

If your Linux distribution offers versions of the Perl modules needed to support Swatch, it is best to get the operating-system-specific ones. Otherwise, you will need to obtain them from either the module's developer's web site or from a centralized repository such as the Comprehensive Perl Archive Network (CPAN). ${ }^{1}$ CPAN indexes most of the Perl packages available, makes the list searchable, and has them available for download. They also have links to the developer's web site if you would prefer to get the modules straight from the source.

To install Swatch you need these modules: ${ }^{2}$

- File::Tail - in File-Tail-0.99.1.tar.gz

- Date::Calc - in Date-Calc-5.4.tar.gz

1 http://www.cpan.org

2 All module version numbers are current at time of printing. 
- Date::Parse - in TimeDate-1.16.tar.gz

- Time::HiRes - in Time-HiRes-1.66.tar.gz

To support these you might also need

- Bit::Vector - in Bit-Vector-6.4.tar.gz

- Carp::Clan - in Carp-Clan-5.3.tar.gz

Once the tar file is on the machine, you must decompress and expand it before it can be installed. Once expanded, read the INSTALL file to make sure that the module has the standard installation commands. For these modules, there is a Perl script called Makefile.PL that creates a makefile specific to the machine. Next, run the make file three times: once to initialize, once to test, and then once to install. After that, the package is ready to use. In order for other users to be able to use these modules, they must be installed by root. The commands follow in shaded boxes (the normal text is what is sent to the console):

tar zxvf perlmod.tar.gz

Lists all the files in perlmod.tar

cd perlmod

perl Makefile.PL

Writing Makefile for Perl: Mod

make

Check for errors

make test

Look for "All tests successful"

make install

Check for errors

Repeat for the other packages and you are ready to install Swatch itself.

\subsubsection{Installing Swatch}

Installing Swatch involves the same procedure as the Perl modules. First, download the tarball to the local machine from http://sourceforge.net/projects/swatch. There is a patch needed to enable the throttle action to fully work. Download that from http://www.cert.org. These instructions are for Swatch 3.1.1 (the normal text is what is sent to the console):

tar zxvf swatch-X.x.x.tar.gz

Lists all the files in swatch-X.X.X.tar

patch - p $\odot<$ throttle.patch 
The character after the $-p$ is a zero

cd swatch-X.X.X

perl Makefile.PL

Writing Makefile for swatch

If Time::HiRes, Date::Calc, or Date: Parse are missing it will say

make

Check for errors

make test

Look for "All tests successful"

make install

Check for errors

Swatch is now ready to be executed. 


\section{Swatch Configuration-Rules 1}

The configuration file contains a list of rules

Default file location is $\$ H O M E / . s w a t c h r c$, but it can be any name and any location

The three parts of a rule:

- Event - "watchfor" or "ignore"

- Pattern - regular expression pattern to look for

- Action - what the script does when the pattern is found

\subsubsection{Swatch Configuration}

The configuration file is the source of Swatch's power. It is a text file of rules that are used to create the script that will be run against the log file. This topic is about how to develop your own configuration file.

Before you begin adding rules, determine what you are trying to find. Perhaps you want to look for Nessus attacks in your Apache log files, or find when people try to use an SMTP server as an open relay. Make a list of strings or regular expression patterns that you might need. Keep in mind that creating a good configuration file is an iterative process, and it may take a few rounds to extract the desired information from the file. 


\section{Swatch Configuration-Rules 2}

Rules are looked for in the order they appear in the configuration

file.

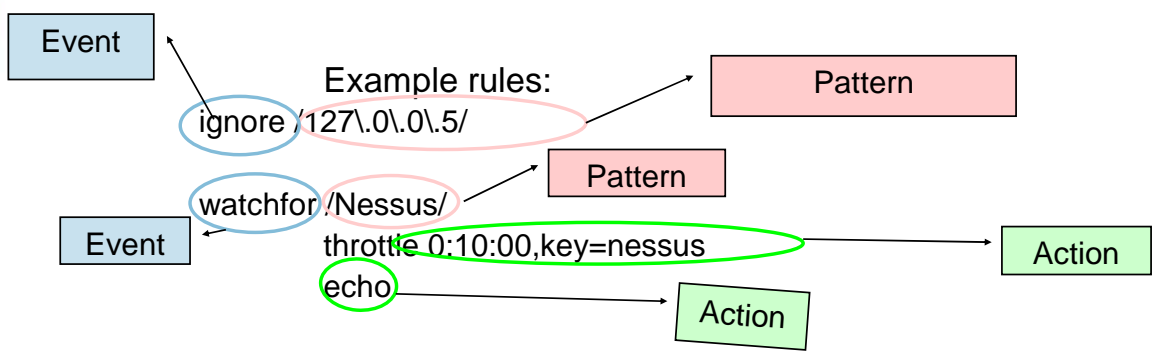

- The first rule looks for the string "127.0.0.5" and ignores any log entries that contain it. The second rule looks for log entries that contain "Nessus" and echoes them to the console, but only at the rate of one entry every 10 minutes.

\subsubsection{The Configuration File Location}

By default, Swatch looks for the configuration file .swatchrc in the home directory. If this file is not found, it uses a default configuration of

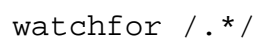

echo

This merely echoes every message in the log file to the console. This is not any more useful than inspecting the log file by hand. To harness the power, a customized configuration file should be created (this is addressed in the next section, 1.1.3.2).

The default name is .swatchrc, but it can be whatever you want. Configuration files for different types of log files may be identified by a distinct name. One might call their Apache configuration file apache.swatchrc and their sendmail sendmail.swatchrc. The file can be stored anywhere that is accessible from the command line, not just in the home directory. If a different name or location is used for the configuration file, it is added as an argument to the command line when Swatch is executed (this is discussed in Section 1.1.4).

\subsubsection{Adding Rules to the Configuration File}

Rules are a list of keywords and values that are used to make conditional statements to check against, and actions to take if one is true. They have three parts: the event, the pattern, and the action(s). 


\subsection{Types of Events}

There are two types of events in Swatch: "watchfor" and "ignore." The keyword "watchfor" looks for the specified pattern in messages in the log file. The "ignore" keyword will skip the rest of the rules when a message matches the pattern.

By default, the first rule that matches a message will be the only rule that acts on that message. This property can be harnessed by using the "ignore" event to filter out messages. For example, since you know that the system administrator always uses the same machine to do penetration testing, you create an "ignore" rule for messages that come from a specific internal IP address, 127.0.0.5, and list the rule for Nessus scans after it so that internal scans will not cause alerts. In this case, you will want to put these two events in this order:

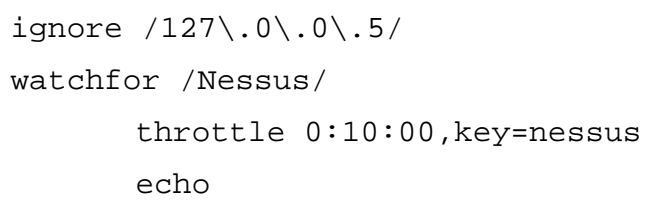

\subsection{Types of Patterns}

The value for the event keyword is the regular expression pattern that follows on the same line. The simplest regular expression is a string to match character by character enclosed in “/”; for example, “/Nessus/” matches only the substring "Nessus” if it appears anywhere in the line. If there are characters in the search string, the capitalization must be the same for the string to match. If you want "nEsSuS" to also match, then you need to put an "i" after the second “/” to indicate a case insensitive search.

\section{Regular Expressions}

A regular expression is a pattern that describes or matches a set of strings [Wikipedia 05d]. It is a syntax for describing more general criteria for matching strings than simply matching a word in a string. For example, with a regular search you could find the substring "cat" in "catapult." But a regular expression would let you look for a string that starts with "ca" and ends with "t," and you would find both "cat” and "catapult."

For more examples of regular expressions in general, see the Wikipedia entry:

http://en.wikipedia.org/wiki/Regular_expression

For more examples of regular expressions in Perl, see the Perl manual page:

http://www.perl.com/doc/manual/html/pod/perlre.html

\subsection{Types of Actions}

Actions are what the script does when it matches a pattern. They range from printing the log message to the console to executing a separate script to call a pager. There can be multiple 
actions associated with each rule. The "ignore" event has its action built in, namely, to stop looking at the log entry. The complete list of actions is found in Table 1 [SourceForge 04]:

Table 1: Actions in Swatch

\begin{tabular}{|c|c|}
\hline Action & Description \\
\hline echo [modes] & $\begin{array}{l}\text { Prints the log message to the console. } \\
\text { Takes an optional value of a text color, a background color, or a } \\
\text { font weight. } \\
\text { Possible values are bold, underscore, inverse, blink, black, } \\
\text { red, green, yellow, blue, magenta, cyan, white, black_h, } \\
\text { red_h, green_h, yellow_h, blue_h, magenta_h, cyan_h, and } \\
\text { white_h. }\end{array}$ \\
\hline bell [n] & $\begin{array}{l}\text { Prints the log message to the console and then rings the bell }(\backslash 007) \\
\mathrm{n} \text { times. }\end{array}$ \\
\hline $\begin{array}{l}\text { throttle } H: M: S \\
{[, \text { key=log } \mid<\text { identifier }>]} \\
{[, \text { time_from=timestamp }]} \\
{[, \text { time_regex }=<\text { regex }>]} \\
{[\text {, threshold=N] }}\end{array}$ & $\begin{array}{l}\text { throttle reduces the number of times an action is performed on } \\
\text { messages matching the same pattern in the specified duration. } \\
\text { Hours, minutes, and seconds must all be specified. However, the } \\
\text { time does not need to be specified if threshold is being used. } \\
\text { The key is the identifier that is stored to compare to new mes- } \\
\text { sages. log means use the exact log file, excluding a syslog time- } \\
\text { stamp, if present, and is the default. Any other string will be used } \\
\text { exactly as requested/indicated. } \\
\text { Setting the time_from option to timestamp indicates that the } \\
\text { time in the log message should be used instead of the system time } \\
\text { for comparisons. This is best for examining a log file. } \\
\text { The time_regex lets you specify a regular expression to match the } \\
\text { timestamp of the message. The default is a regular expression for } \\
\text { the syslog timestamp. } \\
\text { The threshold=N acts on the first instance and on every Nth in- } \\
\text { stance after that. It repeats counting once } \mathrm{N} \text { messages have been } \\
\text { found. Each instance is appended with "(threshold } \mathrm{N} \text { ex- } \\
\text { ceeded)." }\end{array}$ \\
\hline exec command & $\begin{array}{l}\text { Executes the command listed. } \\
\text { If arguments are needed, they may be substituted with fields in the } \\
\text { log message. } \$ N \text { is replaced with the Nth field in the line. A } \$ \odot \text { or a } \\
\${ }^{*} \text { uses the entire message. The - - awk-field-separator switch } \\
\text { must be used during execution (see Section } 1.1 .4 \text { ). }\end{array}$ \\
\hline
\end{tabular}




\begin{tabular}{|c|c|}
\hline Action & Description \\
\hline $\begin{array}{l}\text { mail }[\text { ad - } \\
\text { dresses: bob: joe:...] } \\
{[\text {, subject=Subject }]}\end{array}$ & $\begin{array}{l}\text { Sends an email to the address(es) listed with the subject listed con- } \\
\text { taining the matched log messages. Must have a sendmail compati- } \\
\text { ble server installed. Default recipient is the user who is running the } \\
\text { program. }\end{array}$ \\
\hline $\begin{array}{l}\text { pipe com- } \\
\text { mand [, keep_open] }\end{array}$ & $\begin{array}{l}\text { Pipes the log messages into the command. keep_open keeps the } \\
\text { pipe open until a different pipe is activated or Swatch exits. }\end{array}$ \\
\hline write [user:user:...] & $\begin{array}{l}\text { Uses write(1) to send the message to the users listed. The user } \\
\text { running Swatch is the default. }\end{array}$ \\
\hline continue & Tells Swatch to evaluate this message against the rule set. \\
\hline quit & Causes Swatch to exit. \\
\hline when=day: hour & $\begin{array}{l}\text { This action is a modifier to all the other actions indicating that the } \\
\text { action should occur only during the times specified. For example, } \\
\text { when=1-5- } 8 \text { - } 17 \text { indicates that the action should occur only Mon- } \\
\text { day-Friday between } 8 \text { a.m. and } 5 \text { p.m. }\end{array}$ \\
\hline
\end{tabular}

The exec command can be used to write log entries to a file. Redirection from the console does not work because there are unprintable characters on the command line that are printed in the resulting file and are meaningless and in the way. Use this action:

exec "echo $\$ *>$ output.txt"

When examining a file, the most useful actions will be echo, exec, and throttle. The others are more oriented for when you need to be alerted to a new development in real time.

It is also possible to include Perl in the configuration file if you want to do something such as define a regular expression for repeated use. Start each line with "perlcode" and end it with a semicolon.

The throttle command can be very powerful. It will take some practice to get the right balance of regular expressions to search for timing and to determine whether throttle or threshold is better.

For the regular expressions in the time_regex, all backslashes must be escaped, (e.g., put in two instead of one). Regular expressions on the action line with commas get cut off, so you need to put the expression in a perlcode. This includes both IIS formats. For example:

perlcode my \$iis_time $="(\backslash d\{4\}-\backslash d\{2\}-\backslash d\{2\} \backslash s+\backslash d\{1,2\}: \backslash d\{2\}: \backslash d\{2\} \backslash s) " ;$

watchfor /WEBROOT DIRECTORY TRANSVERSAL/

throttle $\odot: \odot 4: \odot \odot, k e y=w e b, t i m e \_f r o m=t i m e s t a m p, t i m e \_r e g e x=\$ i i s \_t i m e$ echo blue 
Some log formats may not have time_regex. Table 2 lists time_regexes for the log files on popular services:

Table 2: time_regex for Popular Services

\begin{tabular}{|c|c|c|}
\hline Log File & Timestamp & Regular Expression \\
\hline $\begin{array}{l}\text { Apache } \\
\text { access_log }\end{array}$ & 04/Mar/2005:11:38:45 & $(\backslash \backslash d\{2\} \backslash \bigvee \backslash w\{3\} \backslash \bigvee \backslash d\{4\}: \backslash \backslash d\{2\}: \backslash \backslash d\{2\}: \backslash \backslash d\{2\})$ \\
\hline $\begin{array}{l}\text { Apache } \\
\text { error_log; } \\
\text { ssh logs }\end{array}$ & Fri Mar 04 11:38:51 2005 & $(\|w\{3\}\| s+\|w\{3\}\| s+\|\backslash d\{2\}\| s+\|d\{2\}:\| d\{2\}:\|d\{2\}\| s+\| d\{4\})$ \\
\hline $\begin{array}{l}\text { IIS } 6.0 \text { and } \\
\text { later }\end{array}$ & 03/04/05, 11:38:51 & $(\backslash d\{2\} \backslash d\{2\} \backslash \backslash d\{2\}, \backslash s+\backslash d\{1,2\}: \operatorname{ld}\{2\}: \operatorname{ld}\{2\} \backslash \mathrm{s})$ \\
\hline $\begin{array}{l}\text { IIS 5.*, } \\
\text { W3C Ex- } \\
\text { tended }\end{array}$ & 2005-03-14 11:38:51 & $(\backslash d\{4\}-\backslash d\{2\}-\backslash d\{2\} \backslash s+\backslash d\{1,2\}: \backslash d\{2\}: \operatorname{Id}\{2\} \backslash s)$ \\
\hline
\end{tabular}

The when command can be useful for identifying events that occur at abnormal times. Use it to look for login attempts in the middle of the night.

The following is an example configuration file for a syslog setup that has two machines, named "server" and "client," logging to one file. The central syslog server resides on the server. All facilities on the client log to this file. The client is running the default applications. The server logs most facilities to the file and is running Apache (httpd) and Snort, in addition to the basics.

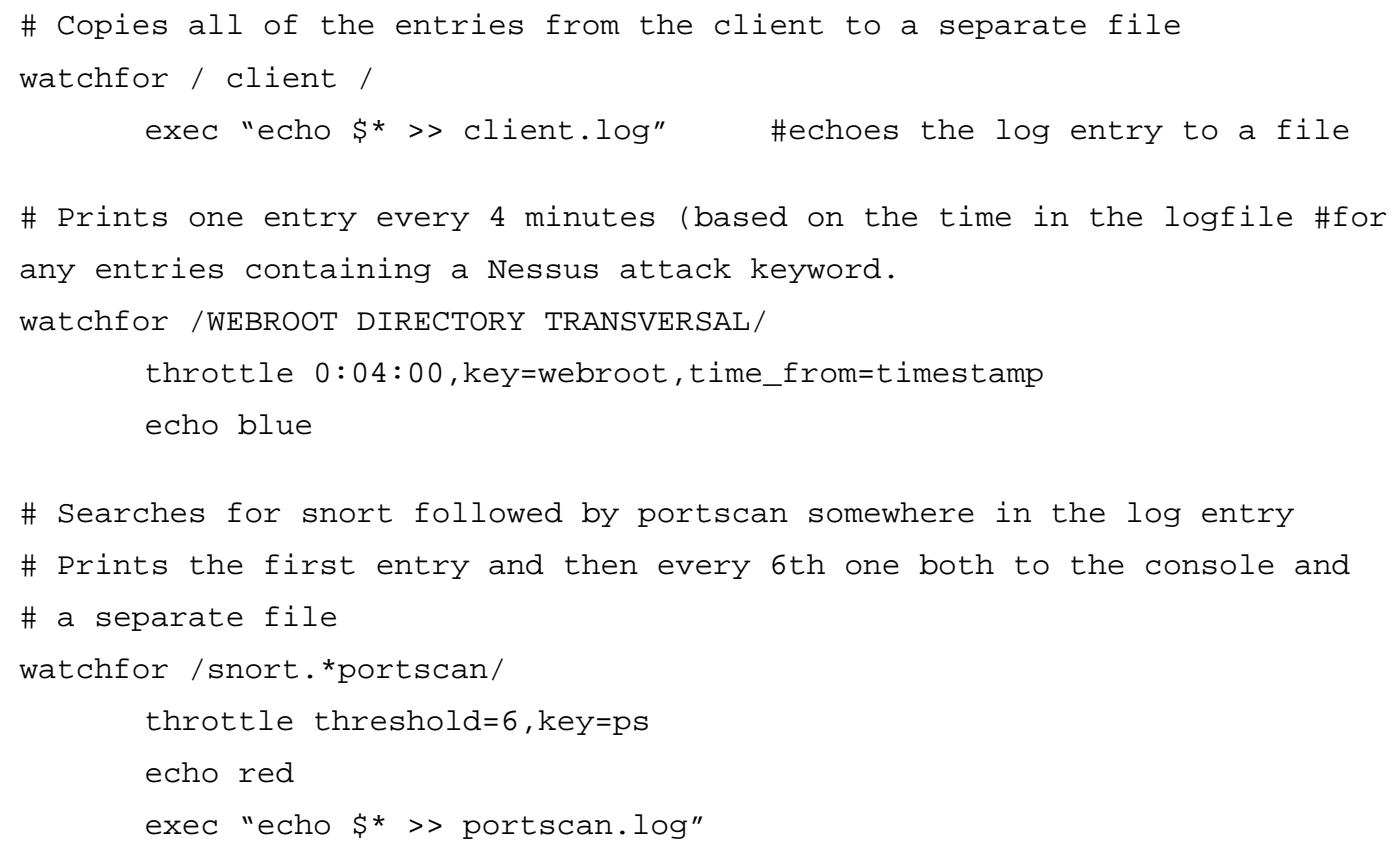


Figure 1 is an example run of the configuration file on a syslog file (“(portscan)” and “(http_inspect)" distinguish the different types of messages).

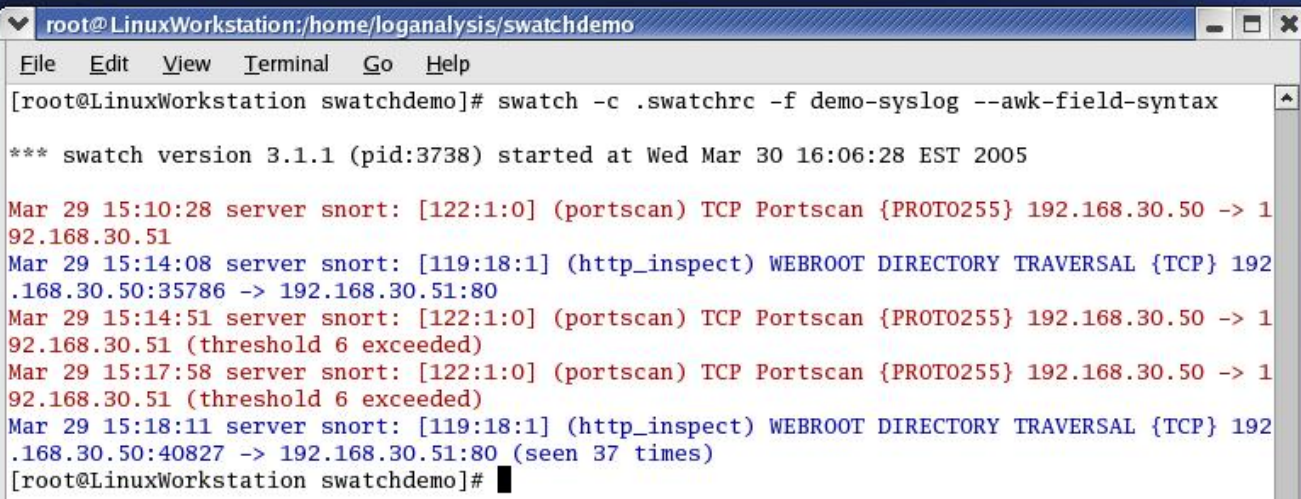

Figure 1: Example Run of the Swatch Configuration File 


\section{Swatch Execution}

$$
\begin{aligned}
& \text { Swatch is run from the command line. } \\
& \text { - swatch }-\mathrm{c} \text {. Swatchrc }-\mathrm{f} \text { file. } 10 \mathrm{~g} \\
& \text { - This runs Swatch using the file .swatchrc as the configuration file } \\
& \text { on file.log. } \\
& \text { - Swatch can be run on only one file at a time, but multiple } \\
& \text { instances of Swatch can be running at once. } \\
& \text { Switch } \quad \text { Use } \\
& \text {-c config.file Name a specific configuration file } \\
& \text {-f file.log } \quad \text { Examine the specific file } \\
& \text {-t file.log } \quad \text { Tail the specific file (/var/log/messages is the default) } \\
& \text {-p command Accepts its input from this command }
\end{aligned}
$$

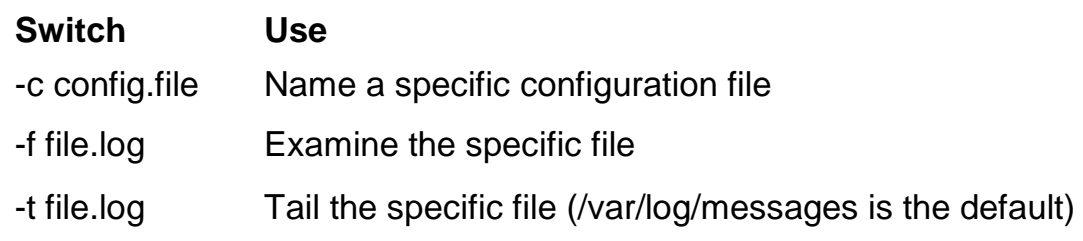

\subsubsection{Swatch Execution}

Swatch is run on the command line and has many options to specialize the execution. You can identify the configuration file, the log file, how to monitor the log file, and even what character(s) indicate a new log message. While most options will be listed, the focus is on the options that relate to running Swatch on a log file in a single pass. More information about all the options can be found in the manual page.

-c filename or --config-file=filename

Indicates where the configuration file is. The default location is $\$ H O M E /$.swatchrc.

- $f$ filename or - -examine=filename

Indicates that Swatch should perform a single pass on the log file.

-t filename or - -tail-file=filename

This option enables Swatch to monitor a file as a service continues to log to it. This action is the default, so if none of -f, -p, or -t is given, Swatch will tail either “/var/log/messages” or “/var/log/syslog."

- p command or - - read - pipe=command

Monitors the data that is being piped in from the given command.

- - awk-field-syntax

Tells Swatch to use the syntax for awk expressions. It is needed when the exec action is used. 
- - input - record-separator=regular_expression

This option indicates that the default record separator of carriage return should be replaced with the regular expression listed.

Other options include - - help and - -version, which respectively give usage information and the current version; - - script - dir=path, which indicates where the temporary script should be stored if not in the user's home directory; and - -restart -time=hh:mm[am | pm], which tells Swatch to restart at a particular time.

The most common usage will be swatch - c . swatchrc -f log_file

Remember to specify the complete path of the configuration file or the log file if either one is not located in the local directory. 


\section{Summary}

Swatch is a simple log monitor designed to run regular expressions against a text log file.

- an effective tool for finding interesting or anomalous events

- configured easily to watch for any type of entry in any type of file

Many actions can be taken on matching message logs, but for analysis "echo" will be used most often.

\subsubsection{Summary}

Swatch can be an effective tool for sifting through log files to find interesting or anomalous events. The results serve as a jumping off point for further inspection of the files by leading you directly to areas of interest. Or it can be used to filter out entries that you know can be excluded so as to reduce the amount of material to examine by hand. Swatch can also be used on other files, such as VMWare virtual disk files and disk images, to efficiently find strings in data. 


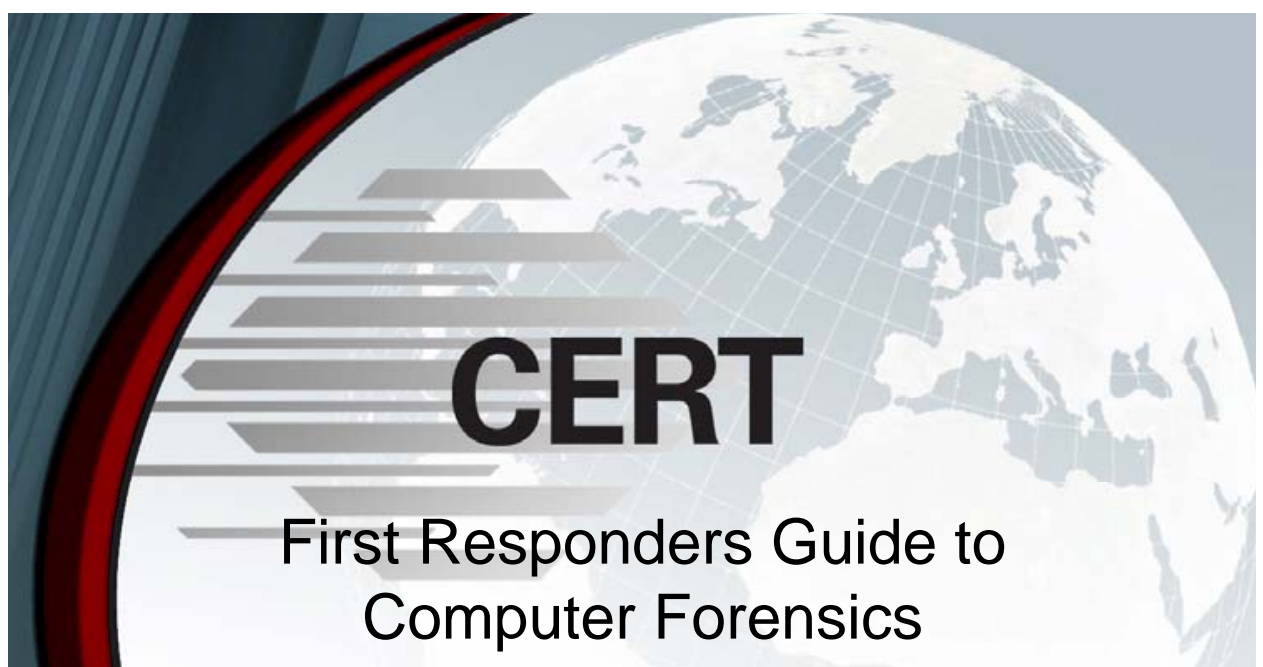

Microsoft Log Parser

\subsection{Microsoft Log Parser 2.2}

This topic is an overview of the installation, configuration, and usage of Microsoft's Log Parser 2.2. The focus is on using Log Parser as a forensic analysis tool, meaning that it will be used on logs after an incident has occurred. If you are planning to use these techniques on files involved in a law enforcement investigation, please make sure you prepare the files according to established best practices. 


\section{Overview}

Why Log Parser?

Installation

Writing Queries

Execution

Forensics in this case is the act of looking for events after they have happened, not performing an investigation for law enforcement 


\section{Microsoft Log Parser 2.2}

Command line tool from Microsoft to process log files using SQL-like queries

Can read in many formats and many types of log files

Output available in many formats-from text to XML files to database storage

Easy generation of HTML reports and MS Office objects

Log Parser functions usable in other scripts

\subsubsection{Microsoft Log Parser Features}

Microsoft Log Parser 2.2 is the most recent incarnation of Microsoft's log analysis tool. Released in January 2005, it contains many improvements and additions to make it useful to anyone with a log file to process. Log Parser is free to download and use. It is a command line tool; there is no GUI to make creating commands easier.

Log Parser uses SQL-like queries to sort through log files. It is very flexible and can be used to read any text based file, file system objects, registries, and database formats.

Log Parser can format the text output into a variety of formats. It can also send output directly to a syslog server, a database, and MS Office charts, and can be used to create HTML reports from a template.

Log Parser can also be used in other programs and scripts to bring its processing power to other applications. 


\section{Log Parser Installation}

Requirements: Windows 2000, 2003, or XP Professional

Download Log Parser from Microsoft's website

Double-click on the setup file and follow the instructions

The Unofficial Log Parser Support Site is an excellent resource: http://www.logparser.com/

\subsubsection{Log Parser Installation}

Log Parser is a Microsoft Windows application that runs on Windows 2000, 2003, and Windows XP Professional. Installation is quick and easy.

Download the file from the Microsoft website. In addition to the Microsoft website, The Unofficial Log Parser Support Site maintains a current link to the setup file on its home page: http://www.logparser.com. That site is also an excellent resource for Log Parser information.

Once you've downloaded the file, double-click on the setup file, LogParser.msi. Follow the instructions in the Setup Wizard and Log Parser is installed. 


\section{Log Parser Input and Output}

Log Parser can read many text-based log formats

- Use the switch - $i$ : TYPE to indicate file type

- Default is determined from the input file type

- Type LogParser - $h$ - $i$ : TYPE for more information on a specific type

Output can be formatted into text files or MS Office objects or sent to other programs

- Use the switch - $:$ TYPE to indicate type of report

- Default is determined from the name of the output file

- Type LogParser - $h$ - ०: TYPE for more information on a specific type

\subsubsection{Log Parser Input and Output}

Log Parser has a variety of built-in text-based formats that it can use to easily parse files and several output formats it can create. Many of them correspond to the log formats of popular applications, though it is Windows-centric.

\subsubsection{Input Formats}

To specify an import format, use the switch - $i$ : TYPE, where TYPE is one of the built-in types. The default input type is determined by Log Parser based on the file extension in the FROM clause. Table 3 lists many of the types and application logs for which each can be used. More information and usage examples for each one can be found by using the command line help: LogParser - $h$ - $i$ :TYPE. Other types can be found under LogParser - $h$.

Table 3: Common Input Formats

\begin{tabular}{|l|l|l|}
\hline Type & Uses & Selected Parameters \\
\hline IISW3C & $\begin{array}{l}\text { IIS W3C Extended Log Format, primar- } \\
\text { ily IIS 5.X logs and older }\end{array}$ & n/a \\
\hline IIS & $\begin{array}{l}\text { Microsoft IIS log format, mostly used } \\
\text { with version 6.0 and newer }\end{array}$ & n/a \\
\hline NCSA & $\begin{array}{l}\text { NCSA Common, Combined, and Ex- } \\
\text { tended Log formats, for Apache logs }\end{array}$ & n/a \\
\hline
\end{tabular}




\begin{tabular}{|c|c|c|}
\hline Type & Uses & Selected Parameters \\
\hline CSV & Text files with comma-separated values & $\begin{array}{l}\text { - headerRow [ON|OFF] - for speci- } \\
\text { fying if there is a header row } \\
\text {-iTsFormat <timestamp format> } \\
\text { - for specifying timestamps other than } \\
\text { "yyyy-MM-dd hh:mm:ss" }\end{array}$ \\
\hline TSV & Tab or space separated values & $\begin{array}{l}\text { - headerRow [ON } \mid \text { OFF ] - for speci- } \\
\text { fying if there is a header row } \\
\text {-iSeparator <character >- } \\
\text { Character that indicates a new field; } \\
\text { can be any character, "spaces," } \\
\text { "space," or "tab" }\end{array}$ \\
\hline W3C & Generic W3C log format & $\begin{array}{l}\text { - iSeparator <character> - } \\
\text { Character that indicates a new field; } \\
\text { can be any character, "spaces," } \\
\text { "space," or "tab” }\end{array}$ \\
\hline XML & XML formatted logs & $\begin{array}{l}\text { - rootXPath }<\text { XPath }>- \text { XPath query } \\
\text { of nodes to be considered roots }\end{array}$ \\
\hline EVT & Windows Event Log & $\begin{array}{l}\text { - fullText [ON|OFF] - Use the full } \\
\text { text message }\end{array}$ \\
\hline NETMON & NetMon captures files & $\begin{array}{l}\text { - fMode [TCPIP / TCPConn ] - Field } \\
\text { mode, each record is a single packet or } \\
\text { a single connection }\end{array}$ \\
\hline REG & Registry keys and values & $\mathrm{n} / \mathrm{a}$ \\
\hline ADS & Active Directory objects & $\begin{array}{l}\text { - objclass <class name>-Spe- } \\
\text { cific class for object mode } \\
\text { - username <uname>- user name for } \\
\text { AD access } \\
\text { - password <pword>-password for } \\
\text { the specified user }\end{array}$ \\
\hline TEXTLINE & Parses entire lines of text files & $\mathrm{n} / \mathrm{a}$ \\
\hline TEXTWORD & $\begin{array}{l}\text { Parses single words out of generic text } \\
\text { files }\end{array}$ & $\mathrm{n} / \mathrm{a}$ \\
\hline FS & File system properties & $\mathrm{n} / \mathrm{a}$ \\
\hline $\mathrm{COM}$ & Custom COM input format & $\begin{array}{l}\text {-iProgID <progid }>\text { - version inde- } \\
\text { pendent Prog ID of the COM plug-in }\end{array}$ \\
\hline
\end{tabular}




\subsubsection{Output Formats}

To specify an output format to create, use the switch - o: TYPE. Output can be in several text formats as well as some non-text ones. The default, for when no INTO clause is stated, is to print the list using NAT to STDOUT. When the INTO clause is stated, if there is no - 0 : TYPE, then Log Parser will attempt to guess from the file extension. Table 4 lists many of the types. More information and usage examples can be found by using the command line help: LogParser - $h$ - o:TYPE. Other types can be found under LogParser - $h$.

Table 4: $\quad$ Output Formats

\begin{tabular}{|c|c|c|}
\hline Type & Format & Special Parameters \\
\hline CSV & Comma-separated values & $\begin{array}{l}\text {-headers [ON | OFF | AUTO] - Write } \\
\text { field names as the first line; AUTO } \\
\text { won't write headers when appending to } \\
\text { an existing file }\end{array}$ \\
\hline TSV & Tab-separated values & $\begin{array}{l}\text {-headers [ON | OFF|AUTO] - Write } \\
\text { field names as the first line; AUTO } \\
\text { won’t write headers when appending to } \\
\text { an existing file } \\
\text { - oseparator <any string> - } \\
\text { Separator between fields: <string>, } \\
\text { "space," or "tab” }\end{array}$ \\
\hline XML & XML output format & $\begin{array}{l}\text { - rootName <element name }>- \\
\text { Name of the Root element }\end{array}$ \\
\hline DATAGRID & $\begin{array}{l}\text { ASP.NET data type for displaying } \\
\text { tabular data }\end{array}$ & $\begin{array}{l}\text { - rpt <number of rows }>- \text { Rows to } \\
\text { print before pausing }\end{array}$ \\
\hline CHART & $\begin{array}{l}\text { Microsoft Office Chart web compo- } \\
\text { nent; MS Office must be installed for } \\
\text { use }\end{array}$ & $\begin{array}{l}\text { - chartType <chart type }>\text { - One } \\
\text { of the designated chart types }\end{array}$ \\
\hline SYSLOG & Sends output to a syslog server & $\begin{array}{l}\text { - host Name <hostname>-syslog } \\
\text { server name }\end{array}$ \\
\hline NAT & $\begin{array}{l}\text { Native format - tabular view of the } \\
\text { records }\end{array}$ & $\begin{array}{l}\text { - rtp <number of rows }>- \text { Rows to } \\
\text { print before pausing }\end{array}$ \\
\hline W3C & W3C Extended Log format & $\begin{array}{l}\text { - rtp <number of rows }>- \text { Rows to } \\
\text { print before pausing }\end{array}$ \\
\hline IIS & IIS Log format & $\begin{array}{l}\text { - rtp <number of rows }>- \text { Rows to } \\
\text { print before pausing }\end{array}$ \\
\hline
\end{tabular}




\begin{tabular}{|l|l|l|}
\hline Type & Format & Special Parameters \\
\hline SQL & Sends the output to a SQL table & $\begin{array}{l}\text {-server <server name }>- \text { Server } \\
\text { the database is on } \\
\text {-database <database name }>- \\
\text { Name of the database }\end{array}$ \\
\hline TPL & $\begin{array}{l}\text { Output a report in a user-specified tem- } \\
\text { plate }\end{array}$ & $\begin{array}{l}\text {-tpl <template file path }>- \\
\text { Path of the template file } \\
\text {-tplHeader <header path> }- \text { Lo- } \\
\text { cation of a header file to use }\end{array}$ \\
\hline
\end{tabular}




\section{Log Parser Queries}

Log Parser can be run with standard SQL queries on the log files

Format:

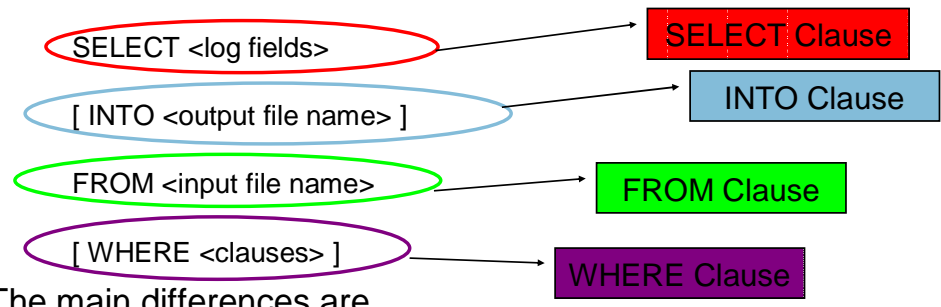

The main differences are

- inclusion of the INTO clause with the output filename

- FROM clause specifies a filename, not a table

Help with SQL can be gotten from Log Parser: LogParser -h GRAMMAR

\subsubsection{Log Parser Queries}

Log Parser queries are based on standard SQL queries. This enables users to be able to select the fields that they need displayed, the log entries to ignore, and which to act on. This also allows for aggregation of log information, such as counting the number of "Error 500" responses from a web server.

The basic parts of a SQL query are the SELECT clause, the FROM clause, and the optional WHERE clause. SELECT indicates the fields to return. FROM indicates the data source to use. And WHERE indicates any conditions for rows to be included in the results.

There are a few differences between standard SQL and the Log Parser SQL. First, the output location needs to be specified with the INTO clause in Log Parser SQL. This is generally a filename, though it might also be STDOUT if displaying the information on the console is desired. Next, the input is from a file, rather than from a table, so a filename follows the FROM clause. Lastly, the input is limited to one file. Joins are not allowed, though subqueries are allowed.

There is query documentation in the help command using LogParser - $\mathrm{h}$ Grammar. There are also a number of examples, both under the help sections for specific input and output formats as well as under a special examples topic: LogParser - $\mathrm{h}$ Examples. For more references on how to write SQL queries, see http://en.wikipedia.org/wiki/SQL. 


\section{Log Parser Query Examples}

\section{Filtering out irrelevant entries}

SELECT *

INTO output.log

FROM input.log

WHERE sc-status $>=400$

Adjusting timestamps

SELECT LogFilename, LogRow, RemoteHostName, RemoteLogName, UserName,

To_Localtime(Add(DateTime, Timestamp('00:00:10', 'hh:mm:ss'))) AS DateTime, Request, StatusCode, BytesSent, Referer, UserAgent, Cookie FROM access_log

\subsubsection{Query Examples}

\subsection{Filtering out Irrelevant Entries}

When focusing on a web server's logs, you will probably want to separate out entries where page requests failed to reduce the logs to the important information. The query

SELECT * INTO output.log FROM input.log

WHERE sc-status $>=400$

would return all of the log entries where the status code was 400 or above, indicating any server errors, "Page Not Found" errors, and access denials. By filtering out what is known to not be a problem, (e.g., successful page requests), it reduces the log files to a more manageable size. Please note that this example is for IISW3C input; different input formats will have different field names.

\subsection{Adjusting Timestamps}

Another common problem is that sometimes the time on one machine may drift and not be the same as on another machine. When you try to compare logs generated on these two machines, events may be out of order. Log Parser can be used to adjust a timestamp in a file. Since we care about the entire log entry and not just the adjusted timestamp, we will need to specify the timestamp and all the other fields. This example uses the NCSA input, is formatted for W3C output, and adds 10 seconds to all the entries.

SELECT LogFilename, LogRow, RemoteHostName, RemotelogName, UserName,

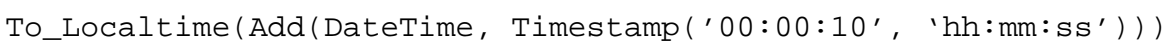


AS DateTime, Request, StatusCode, BytesSent, Referer, UserAgent, Cookie

FROM access_log

This can also be done on IISW3C formatted logs, though the timestamp is different so a different command is needed:

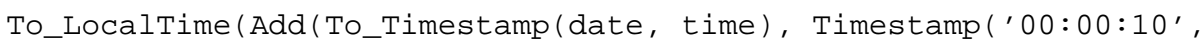
'hh:mm:ss')) )

This query will not change the timestamps in the original files but will create a new file with the adjusted timestamps, making log comparison easier. 


\section{Log Parser COM Objects}

COM allows for cross-platform development. It can be used in $\mathrm{C}++, \mathrm{C \#}$, Visual Basic, Jscript, and VBScript.

COM Objects can be used in two ways:

1. Create custom input formats (the syslog format is not currently supported).

2. Use Log Parser functions in other programs-add queries and input formats to your own scripts.

\subsubsection{Log Parser COM Objects}

Component Object Model (COM) objects allow for cross-platform development of programs and scripts. Log Parser comes with a COM Application Programming Interface (API), allowing programmers to use the underlying constructions of Log Parser either to extend its capabilities or for use in their own programs.

Log Parser COM API is available for use in C++, C\#, Visual Basic, JScript, and VBScript. The only adjustment needed to use the API is that the LogParser.dll binary needs to be registered with the computer's COM infrastructure so the API will be found. Use this command:

\subsubsection{Creating Custom Input Formats}

If the built-in input formats are too restricting, you can create your own. There is one interface to extend in $\mathrm{C}++$ or Visual Basic and another for JScript and VBScript. The resulting script must also be registered with the computer, as in the example above. After that, it can be used when running Log Parser, as in this example:

C: \LogParser>LogParser "SELECT * INTO out.file FROM in.file" -i:COM iProgID: MySample. MyInputFormat

For more examples of this, please refer to the documentation that comes with Log Parser 2.2. 


\subsubsection{Using the Log Parser COM API}

The Log Parser COM API allows for all the same actions as the command line binary, plus more. In addition to query execution and the various input and output formats, it is also possible to have direct access to the log entries in record format.

The MSUtil.LogQuery object is the base object for Log Parser. All others are subclasses. There are subclasses for each of the input and output types, as well as the LogRecordSet and LogRecord types.

There are two modes of query execution: batch and interactive. Batch execution is used when the output will be formatted with one of the output formats, as is done in the command line program. Interactive execution is when no output format is specified and a LogRecordSet is returned. The programmer can then move through the set and process individual records as desired.

The Log Parser documentation provides further illustration of these concepts and specific commands that are available. 


\section{Log Parser Execution}

\section{The Log Parser program opens a command window in the Log Parser directory.}

Log Parser is run in two ways:

1. entering the query at the command line LogParser "SELECT * INTO out.file FROM in.file" i:TYPE -o:TYPE <other switches>

2. using a saved query LogParser file:query.sql -i:TYPE -o:TYPE

\subsubsection{Log Parser Execution}

Log Parser will normally be run on the command line in the special Log Parser window. To start Log Parser go to Start $\rightarrow$ Programs $\rightarrow$ Log Parser $2.2 \rightarrow$ Log Parser 2.2. This opens a command window, displays the help information, and then gives a command prompt with which to work.

On the command line, you list the query, input format, and output format. Certain formats also have other parameters that need to be entered using some extra commands. These commands are listed in Table 5. Switches are separated from their parameters by a colon. The format of the line is

>LogParser "SELECT * INTO out.file FROM in.file" -i:TYPE - o:TYPE

<switches>

Table 5: $\quad$ Misc Log Parser Commands

\begin{tabular}{|l|l|l|}
\hline Command & Function & Parameters \\
\hline$-\mathrm{q}$ & Quiet mode & ON or OFF (default) \\
\hline$-\mathrm{e}$ & $\begin{array}{l}\text { Maximum number of errors allowed be- } \\
\text { fore aborting }\end{array}$ & integer, -1 is default (ignore all) \\
\hline- iw & Ignore warnings & ON or OFF (default) \\
\hline - stats & Display statistics after executing query & ON (default) or OFF \\
\hline
\end{tabular}




\begin{tabular}{|l|l|l|}
\hline Command & Function & Parameters \\
\hline -c & Use built-in conversion query & $\begin{array}{l}-i: \text { TYPE <filename> } \\
\text {-o:TYPE <filename> }\end{array}$ \\
\hline -multiSite & $\begin{array}{l}\text { Send any BIN conversion output to multi- } \\
\text { ple files depending on the SiteID value }\end{array}$ & ON or OFF (default) \\
\hline - saveDefaults & Save options as default values & none \\
\hline - & Restore factory defaults & none \\
\hline -queryInfo & $\begin{array}{l}\text { Display query processing information, but } \\
\text { do not execute query }\end{array}$ & none \\
\hline
\end{tabular}

Queries can get long and it can be cumbersome to type the same one over and over. You can specify a file that contains a query on the command line instead of the query itself. Type file:query.sql instead of the full query. This will be particularly useful for queries that convert from one file type to another (aside from the predefined conversions) and for queries that you run repeatedly. 


\section{Summary}

Log Parser 2.2 is an extremely flexible tool for parsing and searching through logs, Event Viewer files, XML, and other text-based files.

Use it to

- convert logs from one format to another

- filter out specific types of log entries into a new file

- create a new log with an adjusted timestamp

- write custom programs and scripts to process log files

It assumes prior knowledge of SQL.

\subsubsection{Summary}

Log Parser 2.2 is such an improvement on previous versions that it is like a new program. Log Parser is as extensible and flexible as you can make it. It can be used for

- converting log files from one format to another for ease of analysis

- filtering out specific types of log entries into a new log

- creating a new log with an adjusted timestamp after skew has been determined

- $\quad$ writing custom programs and scripts to process log files

There is a steep learning curve with Log Parser. It is necessary to know the fundamentals of SQL queries to be able to process logs effectively. Once this limitation is overcome, many standard logs can be processed and reduced to create meaningful output. 


\section{Module 2: Process Characterization}

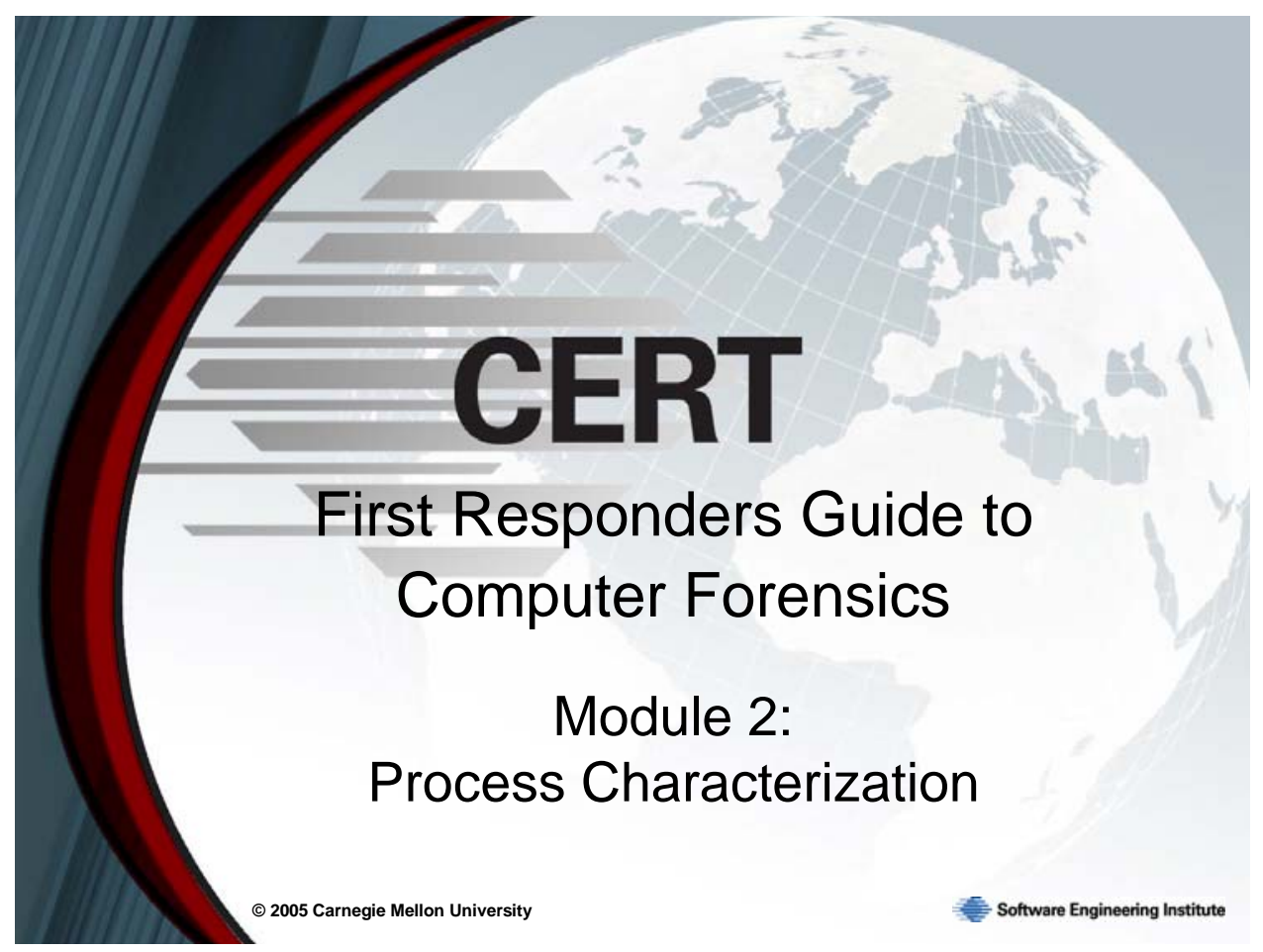

The identification, characterization, and forensic collection of currently running processes on a PC should be a frequently practiced information security procedure. Baselining a running system's processes frequently and enumerating the list of currently running processes will allow you to monitor system activity and see whether serious changes have been made to the system. Running processes on a PC are at the crux of either normal or abnormal system behavior. However, even after process collection, it can be a difficult task to make the determination whether a particular process or set of processes may be the result of an intrusion or malicious user activity.

For most system users and security practitioners, the first alert to abnormal system behavior may be the trivial questions one has about the system during routine day to day interactions with the PC. Why is my PC responding so sluggishly? Why does my PC show extremely high processor activity? What is that process and why is it running? These are commonly asked questions. In most cases, the default reaction to abnormal system behavior is the widely practiced reboot. This second-nature reaction may temporarily solve a problem, but if the machine was truly infected or compromised you may never find the source of the problem because of the volatility of running processes. 


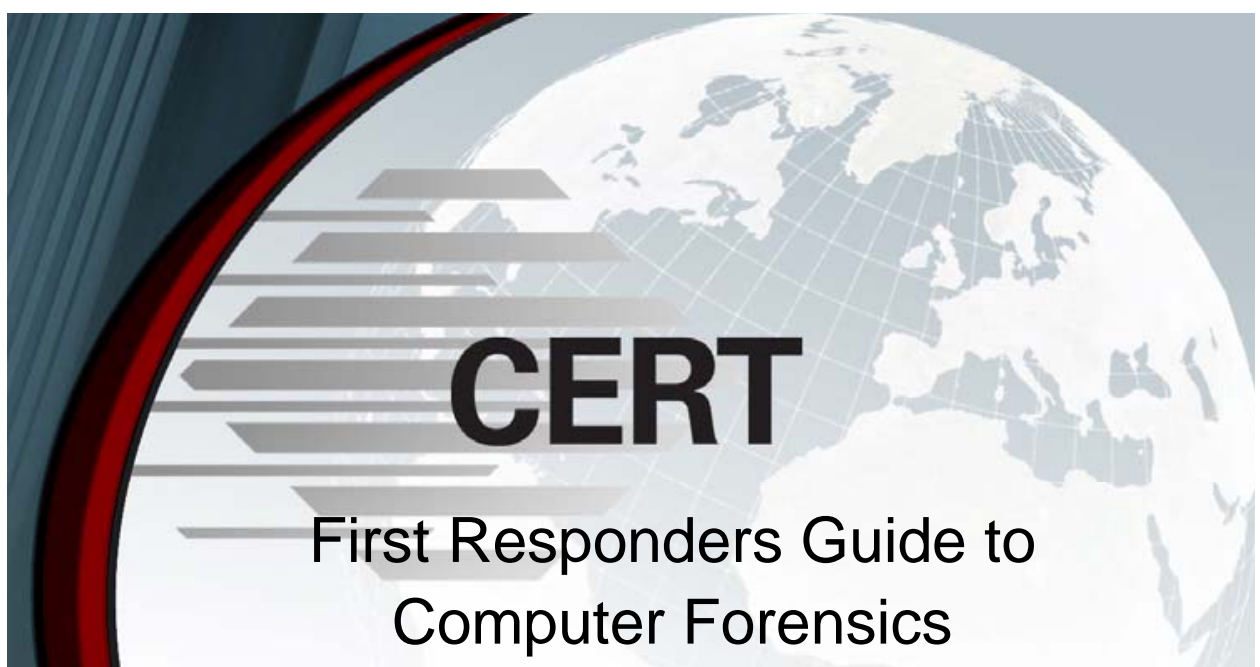

\section{Understanding a Running Process}

○) 2005 Carnegie Mellon University

Software Engineering Institute

\subsection{Understanding a Running Process}

This module is intended to enable system users and first responders (system and network administrators, law enforcement, etc.) to

- better understand running processes

- forensically collect and enumerate the set of current running processes on a system

- potentially differentiate between normal running processes and abnormal running processes (i.e., malware) by looking at key process characteristics 


\section{Objectives}

\section{Background info on processes}

- Programs, processes, threads

- Process tree structure

- Process identifiers (PIDs)

- Process descriptions

- Process analysis checklist

8 key process characteristics

- Tools and native commands for collecting process characteristics

\section{3 process forensic tasks}

\subsubsection{Objectives}

The focus of this module is to demonstrate how to determine the existence of running malware by performing basic process characterization and the forensic examination of running processes on a system. The module is limited to running processes because the majority of malware (viruses, worms, Trojans, backdoors, etc.) have an associated process that may be aliased, newly created, or masked that allows the malware to perform its malicious actions on a machine and sometimes even remain or replicate after a reboot.

Therefore, in an attempt to identify potential rogue processes, we will first cover some background information on processes, identify eight key process characteristics, demonstrate forensic collection procedures, and, finally, introduce native commands and tools that will allow a first responder to forensically collect the key process characteristics for running processes. 


\section{Programs, Processes, Threads}

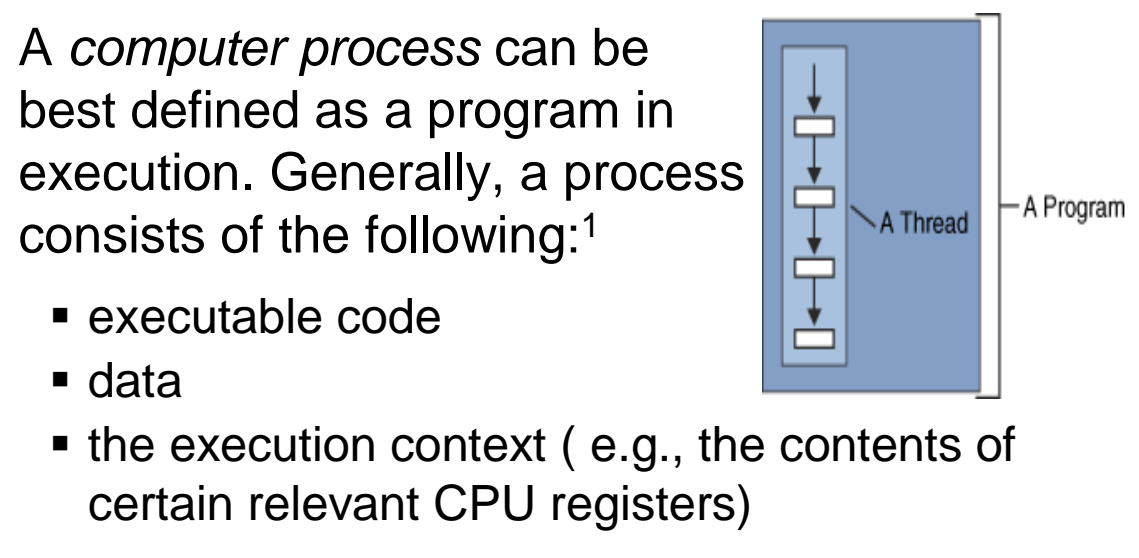

\section{Threads are execution contexts for a process.}

${ }^{1}$ Gollman, Dieter. Computer Security. England: John Wiley \& Sons Ltd, 1999.

\subsubsection{Programs, Processes, and Threads}

A computer process can best be defined as a program in execution. Generally, a process consists of the following: ${ }^{3}$

- executable code

- data

- the execution context (e.g., the contents of certain relevant CPU registers)

While the word "program" refers to the executable code (the exe file, for example), a process is a program that is being executed. When you start a program in Windows, the executable will be loaded into RAM. Windows will then add the new process to its internal process list and make sure the process receives some CPU time as well as memory and other resources. A process can then request any amount of resources from Windows as long as there are resources left. Windows keeps track of which processes are using which resources. As soon as a process is closed or terminated, all resources used by that process will be returned to Windows and will then be handed out to other processes. Unlike memory and similar resources, CPU time cannot simply be requested but is instead shared equally between processes. A process can also return the CPU to Win-

3 Gollman, Dieter. Computer Security. England: John Wiley \& Sons Ltd, 1999. 
dows before the assigned time slice ends. This is actually what happens most of the time and is the reason why your CPU usage is not always at $100 \%{ }^{4}$

The Linux Tutorial ${ }^{5}$ is a great tutorial for understanding in depth how processes work and also provides interactive demonstrations describing the parent-child process relationship.

\subsubsection{Threads}

Threads are execution contexts. Initially each process has a single execution context. This execution context is called a thread. If a process requires another execution context, it can simply create another process. Threads were invented to provide a lightweight mechanism for creating multiple execution contexts. Windows and Linux schedule threads from the operating system with the goal of providing a fair execution environment.

The most obvious distinction between processes and threads is that all threads of a process share the same memory space and system-defined "facilities." Facilities include open file handles (file descriptors), shared memory, process synchronization primitives, and current directory. Because global memory is shared and almost no new memory must be allocated, creating a thread is simpler and faster than creating a process. ${ }^{6}$

\subsubsection{Displaying Threads for a Running Process}

Using the Sysinternals ${ }^{7}$ PsList command line utility with the $-d$ command line argument will display currently running processes, threads for each process, each process's thread state, and memory statistics for each process. This utility comes in very handy when you need a quick way of enumerating all currently running processes, each process's associated threads, and their thread state.

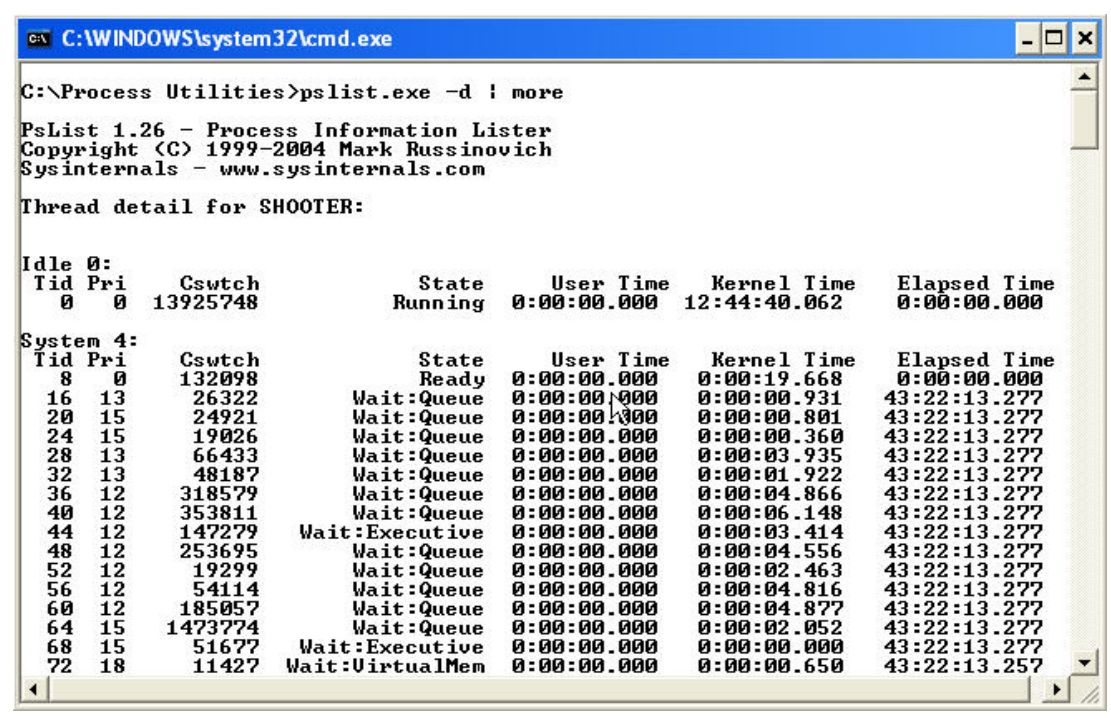

Figure 2: Example Run of PsList

4 http://www.liutilities.com/products/wintaskspro/whitepapers/paper8/

5 http://www.linux-tutorial.info/modules.php?name=Tutorial\&pageid=3

6 Bradford, Edward "High-Performance Programming Techniques on Linux and Windows 2000," http://www.developertutorials.com/tutorials/linux/run-time-linux-windows-050428/page1.html.

7 http://www.sysinternals.com/index.html 


\subsubsection{Sysinternals Process Explorer}

Another Sysinternals utility called Process Explorer is an excellent administrative tool for showing a dynamic display of real-time system process activity. Process Explorer's graphical user interface (GUI) is a more robust Windows Task Manager. It displays in an easy to read format what handles each process has open and what DLLs and memory-mapped files each process has loaded, and has a quick search capability to locate a particular DLL or handle for the currently running processes. ${ }^{8}$

Figure 3 is an action screenshot of the Process Explorer GUI. The GUI displays a process list (e.g., tree format) of currently running processes in the top half of the pane, while the bottom half of the pane displays all currently loaded DLLs for the highlighted mozilla.exe process.

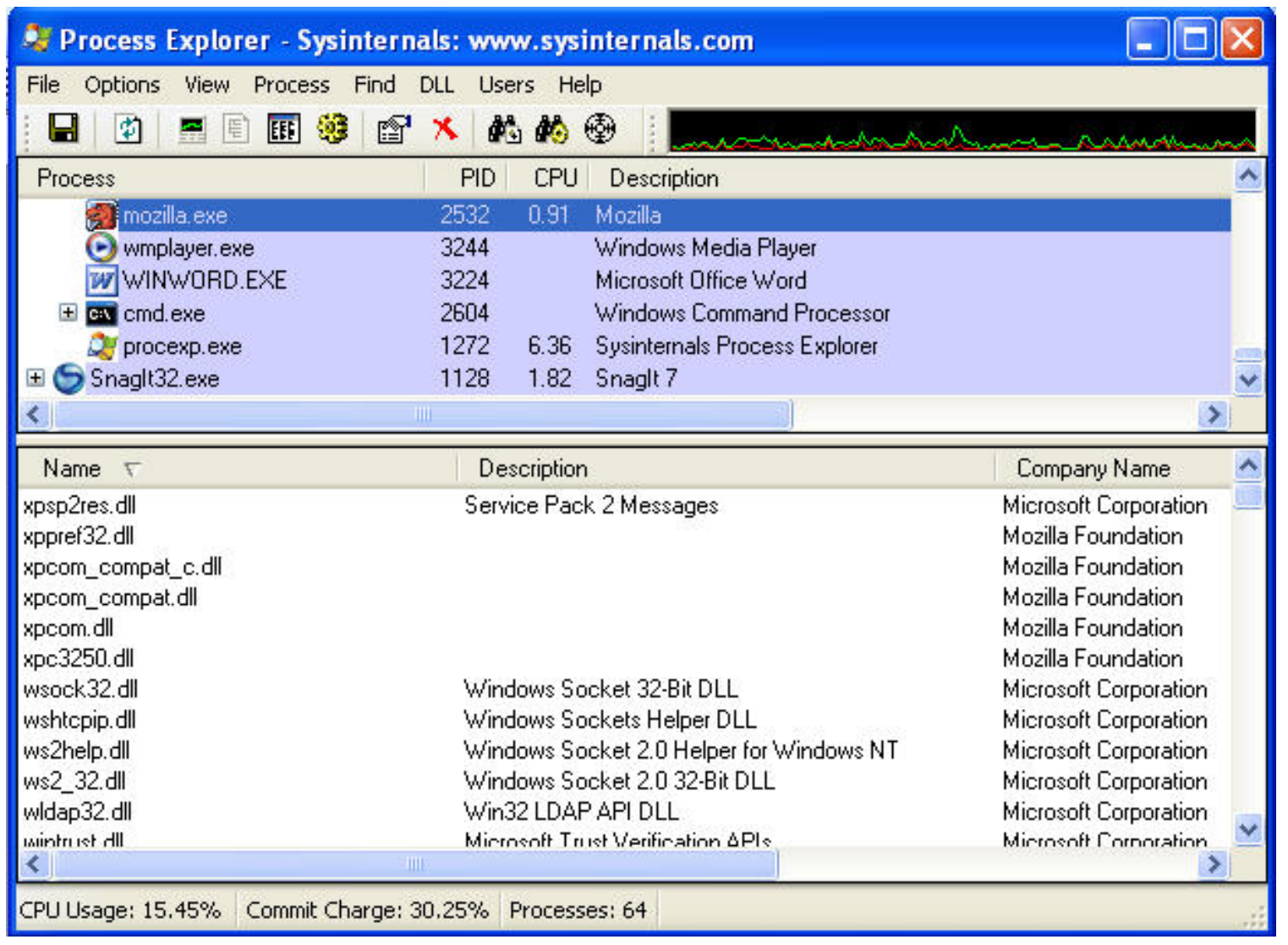

Figure 3: Sysinternals Process Explorer Utility

8 http://www.sysinternals.com/ntw2k/freeware/procexp.shtml 
More importantly, the Process Explorer utility has a unique security feature that verifies a process's image (i.e., the program/binary responsible for the executing process).

If you are curious about whether a particular running process is a legitimate Microsoft process, you could verify the process image by using the added functionality in Process Explorer.

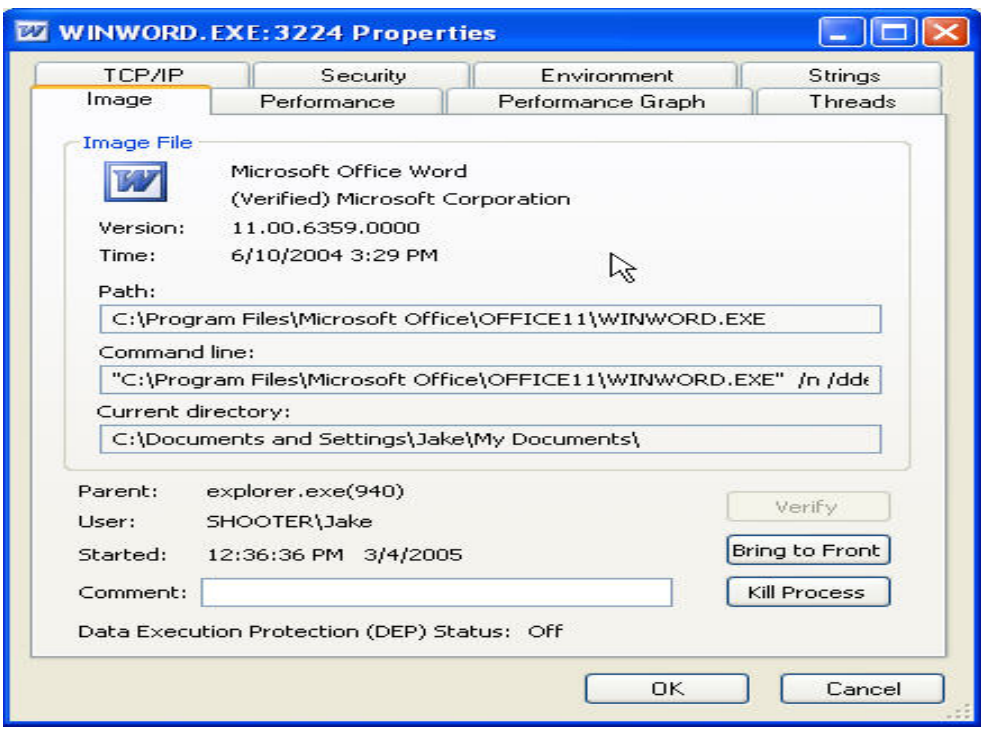

Figure 4: Verifying a Process Image in Process Explorer

To do this, you would

1. Right-click on any of the displayed processes in the Process Explorer GUI.

2. Click Properties.

3. Click Verify. An example result is shown in Figure 4.

In addition to the Verify option, another great feature is the Strings tab.

Clicking on the Strings tab will display all alpha and numeric strings found in the process image, as shown in the example in Figure 5. This may come in handy for looking further into an unknown process image.

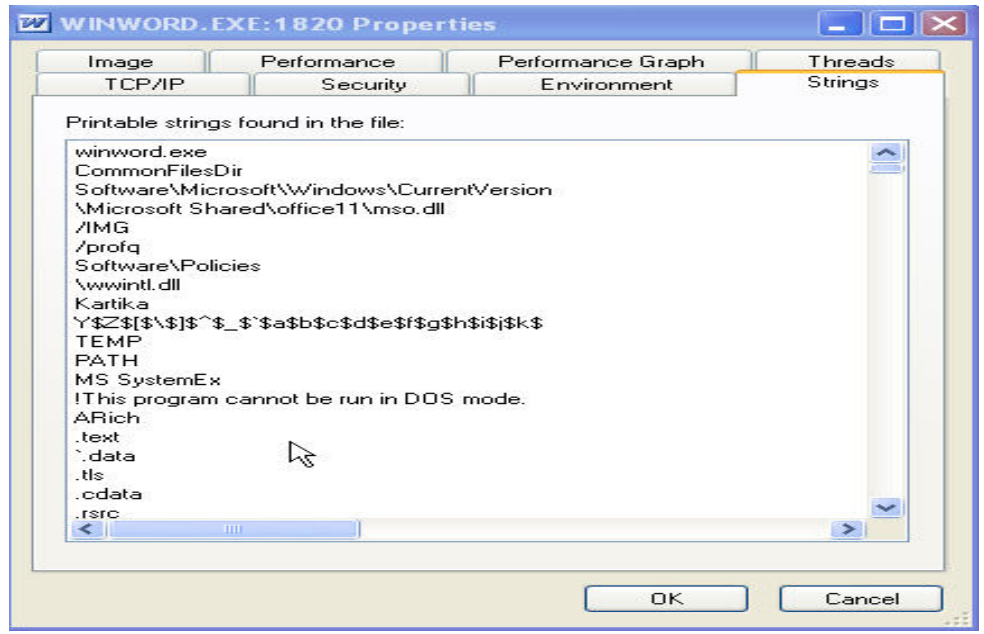

It is important to point out that this utility uses a GUI

Figure 5: The Strings Tab in Process Explorer and, therefore, is not an ideal tool that a first responder would use in responding to a computer security incident to forensically analyze running processes on a machine. This utility should have already been incorporated into the daily operations for everyday system and network troubleshooting. If you were to use the Process Explorer utility in an incident response situation you might actually contaminate potential uncollected evidence from a possibly compromised machine (e.g., changing Mac Times on critical files or folders on the system). Sysinternals' command line utility pslist.exe is a better choice for responding to 
computer security incidents and forensically collecting running processes, simply because it is much lighter and leaves a significantly smaller footprint. 


\section{Process Tree Structure and PIDs}

\begin{tabular}{|c|c|c|}
\hline \multicolumn{2}{|c|}{ ci C:IWINDOWSLsystem32land.exe } & $-\square x$ \\
\hline \multicolumn{3}{|c|}{ C: $\backslash$ Docunents and Settings $\backslash$ Jake $\backslash$ Desktop $\backslash$ Demo 2 Proc } \\
\hline \multicolumn{3}{|c|}{$\begin{array}{l}\text { PsList 1.22 - Process Infornation Lister } \\
\text { Copyright (C) 1999-2002 Mark Russinovich } \\
\text { Sysinternals - ww.sysinternals.con }\end{array}$} \\
\hline \multicolumn{3}{|c|}{ Process infornation for SHOOTER: } \\
\hline Name & Pid Pri Thd & Hnd \\
\hline Idle & $\begin{array}{lll}0 & 0 & 1\end{array}$ & 0 \\
\hline System & $\begin{array}{lll}4 & 8 & 64\end{array}$ & 428 \\
\hline snss & $998 \quad 113$ & 21 \\
\hline csrss & $\begin{array}{lll}968 & 13 & 11\end{array}$ & 714 \\
\hline vinlogon & $996 \quad 13 \quad 24$ & 531 \\
\hline ati2euxx & $668 \quad 8 \quad 5$ & 68 \\
\hline services & 104099 & 337 \\
\hline suchost & 20888 & 85 \\
\hline wdfngr & $\begin{array}{lll}304 & 8 & 4\end{array}$ & 65 \\
\hline suchost & $\begin{array}{lll}428 & 8 & 15\end{array}$ & 220 \\
\hline spoolsu & $\begin{array}{lll}868 & 8 & 15\end{array}$ & 183 \\
\hline ibnpnsuc & 122088 & 37 \\
\hline ati2euxx & 124485 & 66 \\
\hline suchost & $\begin{array}{lll}1268 & 8 & 19\end{array}$ & 219 \\
\hline hpoevmb8 & $\begin{array}{lll}628 & 8 & 8\end{array}$ & $143 \nabla$ \\
\hline 1 & & 1 \\
\hline
\end{tabular}

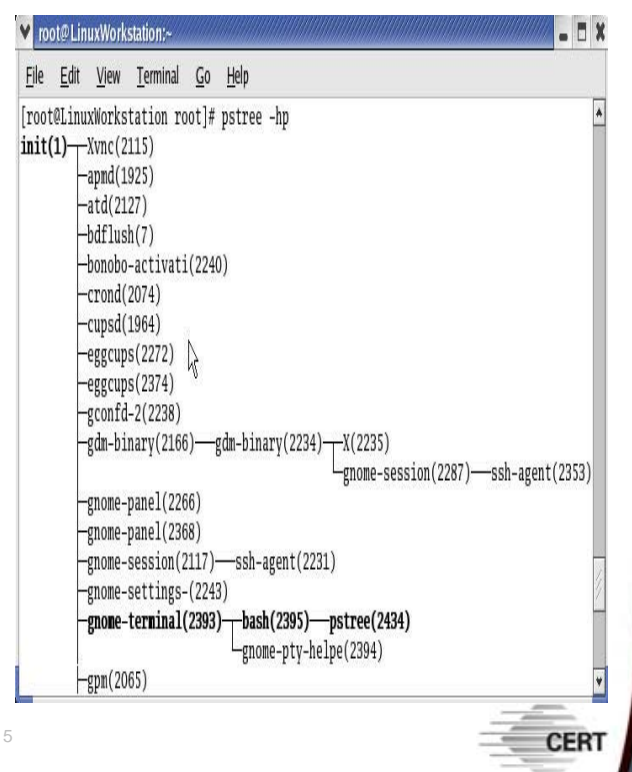

\subsubsection{Process Tree Structure}

Windows and Linux OS environments currently running processes exhibit a hierarchical tree structure. By looking at this hierarchical tree structure of currently running processes we can gain insight about what processes have started other processes and so forth.

This kind of relationship is known as the parent and child process relationship. The creator is called the parent process, the created is called the child process, and the parent-child relationship is expressed by a process tree.

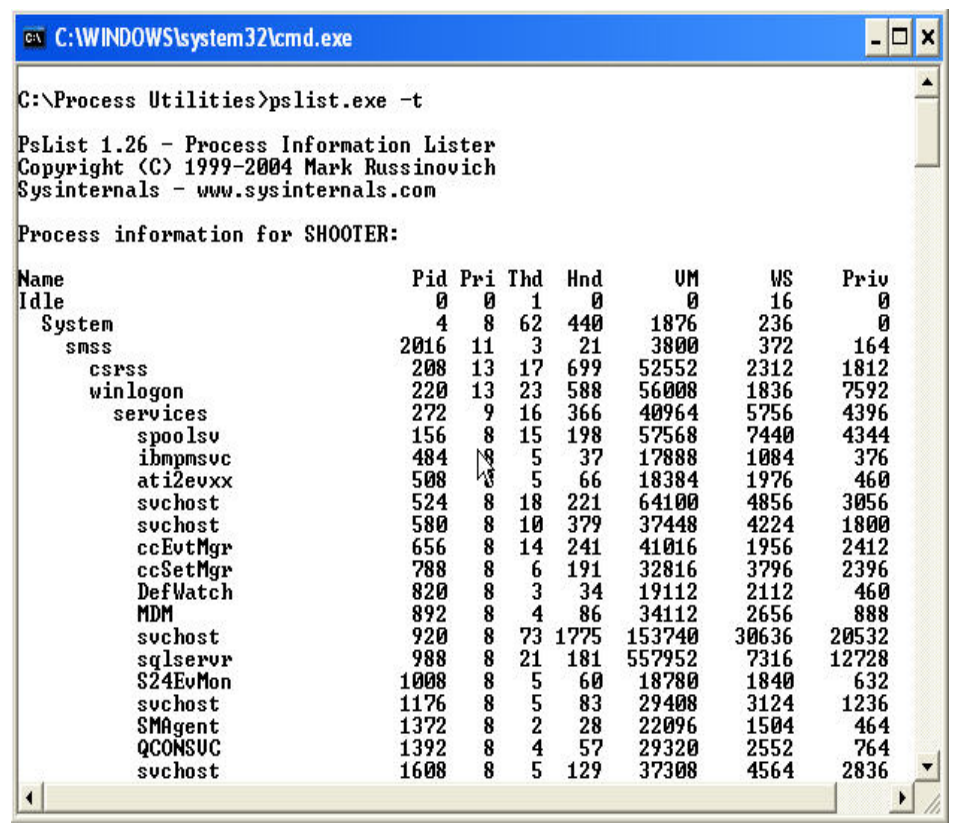

Figure 6: Displaying a Process Tree Using PsList

Using Sysinternals’ PsList utility with the -t command line argument, we can visually display the parent-child relationship (i.e., the process tree) for currently running processes. 
In the example shown in Figure 6, we can easily see that the System.exe process is the parent process for smss.exe and so forth as you work your way down the process tree.

\subsubsection{1 pstree (Linux)}

Using the native Linux pstree command, we can easily display the parent-child process relationship for currently running processes. Using pstree with the -hp command line argument will display the process tree, highlight the current process, and display the PIDs for each process (Figure 7).

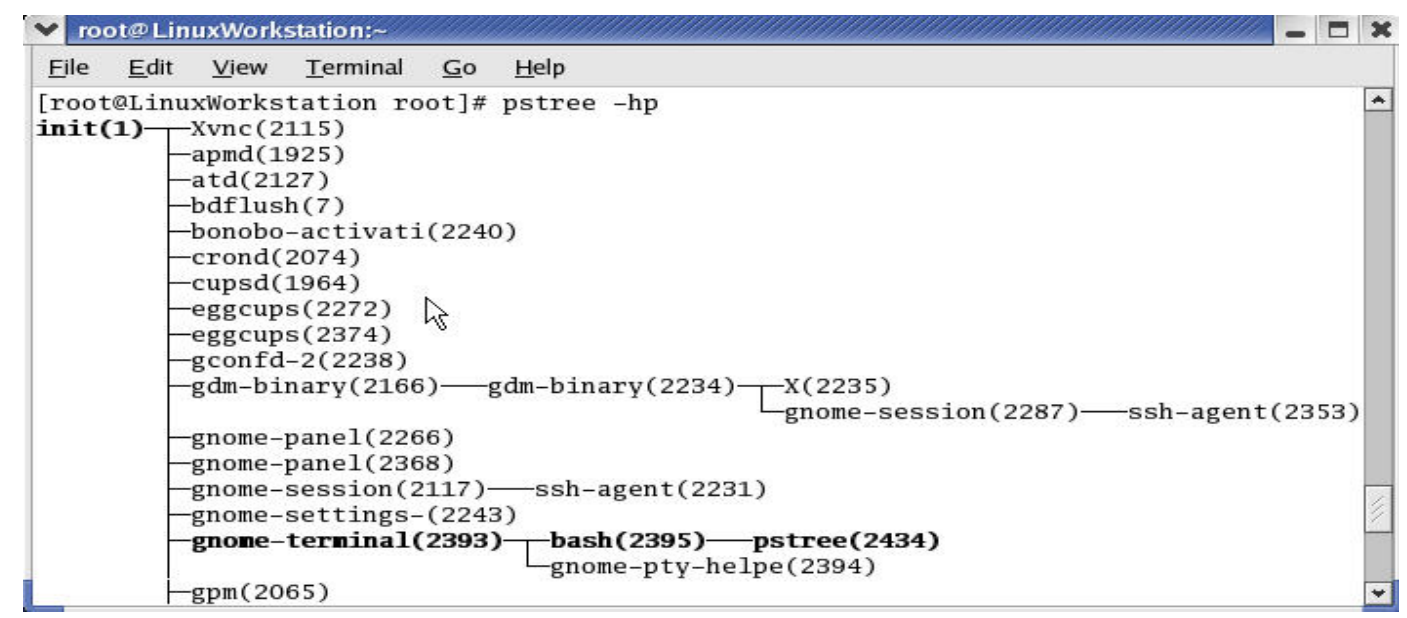

Figure 7: Displaying a Process Tree Using pstree

\subsection{PIDS}

Process identifiers, commonly known as PIDs, are unique integer values assigned to each currently running process. Generally, PID assignments will be multiples of 4 for Windows operating systems, thus guaranteeing an even integer PID assignment ranging from 0 XXXX.

From a forensic or first responder perspective, PIDs offer a quick and easy way of uniquely identifying running processes. However, there is nothing of forensic value in distinguishing legitimate processes from non-legitimate processes by just looking at the PID assignments. What is useful is the mapping or correlation from the PID assignments to generated system event log tickets. We can search the system event logs for a PID that was responsible for generating a certain ticket's event log and map them back to the PID or current running process.

\subsubsection{Linux ps -A}

Using Linux's native ps command, we can quickly display each currently running process's PID assignment and the command that was to used start the process. When displaying processes in Linux, PIDs will always be displayed in sequential order ranging from the infamous init process, or PID 1, to XXXX (Figure 8). 


\begin{tabular}{|c|c|c|c|c|}
\hline \multicolumn{3}{|c|}{$\checkmark$ root@LinuxWorkstation: - } & \multirow[t]{2}{*}{ - $\square$} & $\mathbf{x}$ \\
\hline File & Édit & View Ierminal Go $\underline{\text { Help }}$ & & \\
\hline \multicolumn{3}{|c|}{ [root@LinuxWorkstation root]\# ps -A } & & $A$ \\
\hline PID & TTY & TIME CMD & & \\
\hline 1 & $?$ & $00: 00: 05$ init & & \\
\hline 2 & $?$ & $00: 00: 00$ keventd & & \\
\hline 3 & $?$ & 00:00:00 kapmd & & \\
\hline 4 & ? & 00:00:00 ksoftirqd/0 & & \\
\hline 7 & ? & 00:00:00 bdflush & & \\
\hline 5 & ? & $00: 00: 00$ kswapd & & \\
\hline 6 & ? & $00: 00: 00$ kscand & & \\
\hline 8 & ? & 00:00:00 kupdated & & \\
\hline 9 & $?$ & 00:00:00 mdrecoveryd & & \\
\hline 18 & $?$ & 00:00:00 kjournald & & \\
\hline 73 & ? & 00:00:00 khubd & & - \\
\hline 1375 & ? & 00:00:00 kjournald & & \\
\hline 1825 & $?$ & $00: 00: 00$ syslogd & & - \\
\hline
\end{tabular}

Figure 8: Displaying PID Assignments Using ps 


\title{
Process Descriptions
}

\author{
WinTasks Process Library
}

- Great resource for knowing the exact purpose and description of every single Windows process

Categories of Windows processes

- Top system processes

- Top application processes

- Top security threat processes

- Other unfamiliar processes

\section{Example: svchost.exe}

\subsubsection{Process Descriptions}

There is no easy way of quickly knowing whether the current set of running processes are normal, especially if you do not have in-depth knowledge of the system you are analyzing or if proper process baselining was not implemented. Understanding what each process is and why it is currently running can be a difficult task. There are a few online resources outlined below that will attempt to alleviate some of the ambiguity of unfamiliar Windows processes, particularly in determining whether a process is legitimate.

Uniblue has an online hyperlinked table for each type of Windows process. The online resource is a great tool for quickly checking and gathering information about a known rogue process or gaining information such as a description about any legitimate Windows process. A Uniblue hyperlinked table for each of the following process categories is available online. ${ }^{9}$

\section{Categories of Windows Processes}

- top system processes

- $\quad$ top application processes

- top security threat processes

- other, unfamiliar processes

Figure 9 is a snapshot of the type of information you can obtain from the Uniblue website for each of the categories of Windows processes. We chose a process description pertaining to the svchost.exe process that is often found running on Windows systems.

9 http://www.liutilities.com/products/wintaskspro/processlibrary/allprocesses/ 


\title{
WinTasks Process Library featured in
}

\author{
svchost - svchost.exe - Process Information
}

Process File: svchost or svchost.exe

Process Name: Micros oft Service Host Process

\section{Description:}

svchost.exe is a system process belonging to the Microsoft Windows Operating System which handles processes executed from DLLs. This pirogram is important for the stable and secure running of your computer and should not be terminated. Note: svchost.exe is a process which is registered as the W32.Welchia.Worm. It takes advantage of the Windows LSASS vulnerability, which creates a buffer overflow and instigates your computer to shut down. To see more information about this vulnerability please look at the following Micros oft bulletin: $\mathrm{http} / /$ www.microsoft.com/technet/security/bulletin/ms04011.mspx This is a registered security risk and should be removed immediately. Please see additional details regarding this process

\section{Figure 9: WinTasks Process Description}

\subsubsection{Process Hashes (National Software Reference Library)}

Another valuable online resource is NIST's National Software Reference Library's repository of SHA1 and MD5 hashes for critical system and application files. The NSRL repository includes hashes of non-English software files, operating system files, application software files, images, and graphics found on a typical Windows installation. NSRL's stored repositories of hashes are cryptographic hashes of safe or uncorrupted files. This is very important when wanting to compare your own system's critical file hashes against a known safe set. This online resource provides the cryptographic hashes free of charge; they are downloadable as ISO images.

Once you've burned the images to a CD-ROM, you can unzip the zipped files and get started. You'll find a list and description of the five text files that come with the NIST Operating System ISO. The Operating System ISO is important because it contains safe hashes of executables and DLL files. These are common types of critical files on Windows machines that become corrupted or replaced with compromised ones. NSRL maintains and updates the ISO images periodically (non-English software, operating systems, application software, and images and graphics) as new updates and patches are released.

Operating System ISO:

- Hashes.txt - contains hashes for the files so you can check the integrity of the downloaded files

- Version.txt - displays the version and date of the downloaded ISO. Keep in mind these ISOs will be updated periodically, so it is import to check the version file for each ISO file because hashes may change with different versions. 
- NSRLMfg.txt - displays the Manufacture Code (MfgCode) and Name (MfgName) of the supported manufacturers

- NSRLProd.txt - displays the supported "ProductCode," "ProductName," "Product Version,” "OpSystemCode," "MfgCode," “Language,” and “ApplicationType”

- NSRLFile.txt - This is the file that actually has the list of the SHA1 and MD5 hashes that can be used for checking critical associated OS files. The format of each entry in the text file goes as follows:

SHA-1, MD5, CRC32, FileName, FileSize, ProductCode, OpSystemCode, SpecialCode

Here is an example of one entry in the NSRLFile.txt text file that contains a list of SHA-1 and MD5 hashes. As you can see, the entry corresponds to the mshearts.exe program for a Windows XP machine and has a SHA-1 and MD5 hash for the mshearts executable.

"001A6C9B8D9471B0A3B4F46302DB951F4D877227","BE1B85306352E0AC901EC0850 6792B6B","CB76D275","mshearts.exe",126976,1567,"WINXP","' 


\section{Process Analysis Checklist}

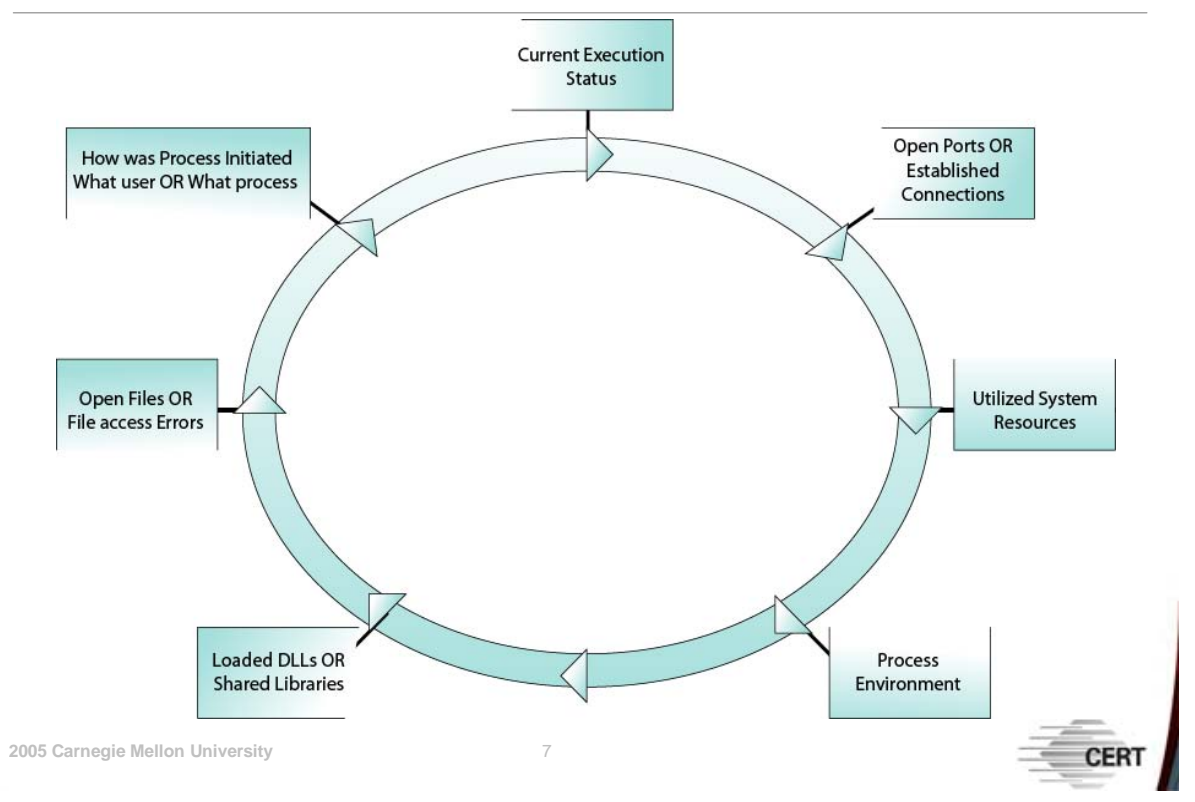

\subsubsection{Process Analysis Checklist}

The procedure of inspecting processes for unexpected behavior or characteristics involves many detailed actions. The abbreviated checklist below contains questions about processes that you may wish to ask yourself and characteristics you may wish to enumerate. In the following paragraphs we are going to demonstrate how to forensically collect some identified items and some extra process characteristics using native commands and third-party tools.

- How was this process initiated?

- By what user?

- From what program or other process?

- What is the current execution status of each process?

- Is it running, stopped, suspended, swapped out, exiting, or in some other unexpected state?

- Does the process continue to appear among active processes after it should have exited?

- Is it missing from among the processes you expected to be active?

- In what environment is this process executing?

- What system settings are in effect for this process?

- Did the process inherit any environment settings from other processes?

- How might the current environment settings affect how the process operates and what it can access?

- With what options or input arguments is the process executing? Are these appropriate settings? 
- Are the system resources (CPU time, memory usage, etc.) being utilized by each process within expected consumption amounts?

- Are there any processes that seem to be tying up an unusually large amount of system resources?

- Are any processes not performing as expected because they don’t seem to be getting enough resources?

- What is the relationship between this process and other processes executing on the system? What are the characteristics of the related processes?

- What files have been opened by the processes executing on the system?

- Are they authorized to have these files open?

- Have the files been opened with excessive privileges (e.g., opened with read-write capability when there is no reason for the process to write to the file)?

- Have there been any unexpected accesses to sensitive system files or other private data, such as password files?

- From what process were the accesses made?

- With which user is that process associated?

- Have there been any unauthorized attempts to access a file? Has the system reported any file access errors? 


\section{Process Characteristics}

1. Process filename

2. Open ports

3. Open files

4. Base priority

5. Start, stop, elapsed times

6. Location (i.e., full path) of process image

7. Survivable processes

8. Loaded DLLs or libraries

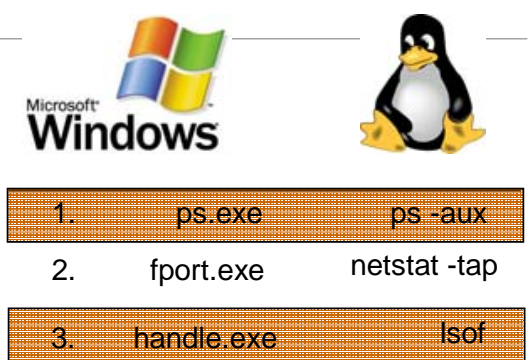

4. pslist.exe top $-\mathrm{n} 1$

\begin{tabular}{|c|c|c|}
\hline 5. & Wisliexe & ps-auxw \\
\hline & psloglistiexe & \\
\hline 6. & listdlls.exe & Isof $-p$ PID \\
\hline 7. & autorunsc.exe & checkconfig. \\
\hline & & 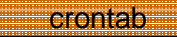 \\
\hline
\end{tabular}

8. listdlls.exe

Idd

\subsubsection{Common Process Characteristics}

We have outlined the eight key process characteristics that follow along with the Process Analysis Checklist, as well as a set of tools and native commands a first responder can use to collect those characteristics. Note that this is not an exhaustive list of running process characteristics. But collecting these eight process characteristics for a potential rogue can significantly aid in determining whether that running process is legitimate or not.

\subsubsection{Process Filenames}

The process filename is the filename of the process image that was executed to initiate the currently running process. In most cases you can look at the process filename to determine whether the current running process is a legitimate Windows or Linux process. 


\subsubsection{1 pulist (Windows)}

Using the pulist.exe command line utility, a Windows Resource kit utility, we can generate a list of currently running processes and the associated filenames to be examined for unexpected process filenames and unusual user identifications.

In the screenshot in Figure 10, the four processes that we identified to be rogue processes judg-

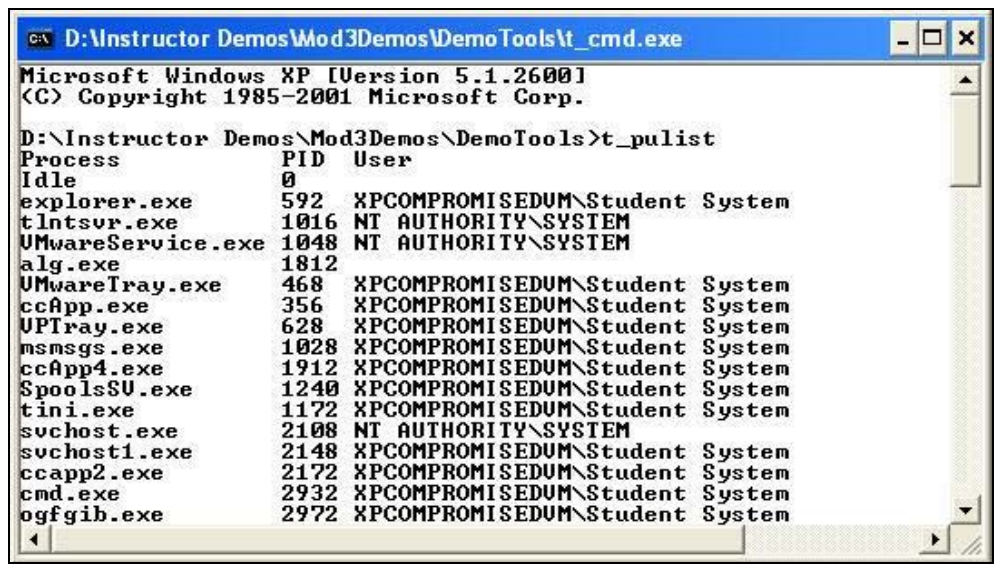

Figure 10: Listing Process Filenames Using pulist ing by the process filenames are tini.exe, klogger.exe, svchost1.exe, and qfskrtwj.exe.

\subsubsection{2 ps (Linux)}

The Linux ps command can be used to display the filename of the process image as well as other things outlined in Table 6 and Table 7.

Table 6: $\quad$ A Subset of ps Options

\begin{tabular}{|l|l|}
\hline Option & Description \\
\hline \$ps -ux & View current processes \\
\hline$\$$ ps -U user & View other system users running processes \\
\hline$\$$ ps -C program_name & View all occurrences of a program \\
\hline$\$$ ps -p4, 8, 2203 & View selected processes 4, 8, 2203 \\
\hline$\$$ ps -efww & View all processes with full command lines \\
\hline
\end{tabular}

Table 7 describes some output headings for $p s$ and top output.

Table 7: $\quad$ Output Headings for ps and top

\begin{tabular}{|l|l|}
\hline Field & Description \\
\hline USER & Username of the process owner \\
\hline PID & Process ID \\
\hline \%CPU & Percentage of the CPU this process is using \\
\hline \%MEM & Percentage of real memory this process is using \\
\hline VSZ & Virtual size of the process, in kilobytes \\
\hline
\end{tabular}




\begin{tabular}{|l|l|}
\hline Field & Description \\
\hline RSS & \\
\hline TT & $\begin{array}{l}\text { Current Process Status } \\
\text { R= Runnable } \quad \text { D = In disk wait } \\
\text { I = Sleeping ( }<20 \text { sec) } \quad \text { S = Sleeping }(>20 \text { sec) } \\
\text { STAT }=\text { Stopped } \quad \text { Z = Zombie } \\
\text { Additional Flags } \\
\text { L = Some pages are locked in core (for rawio) } \\
\text { S = Process is a session leader (head of control terminal) } \\
\text { W = Process is swapped out } \\
+=\text { Process is in the foreground of its control terminal }\end{array}$ \\
\hline START & Time the process was started \\
\hline TIME & CPU time the process has consumed \\
\hline COMMAND & Command name and arguments \\
\hline
\end{tabular}

\subsubsection{Open Ports}

Another critical process characteristic is the number of ports a particular process has open. Processes that have unfamiliar or unnecessary TCP or UDP ports open could indicate that the process is a backdoor or Trojan allowing remote access to the machine.

\subsubsection{1 fport (Windows)}

The fport.exe command line utility, like the native windows netstat -anb command, displays all open TCP/IP and UDP ports and maps them to the owning application as shown in Figure 11.

Fport also maps those ports to running processes with the PID, process name, and path to the process image.

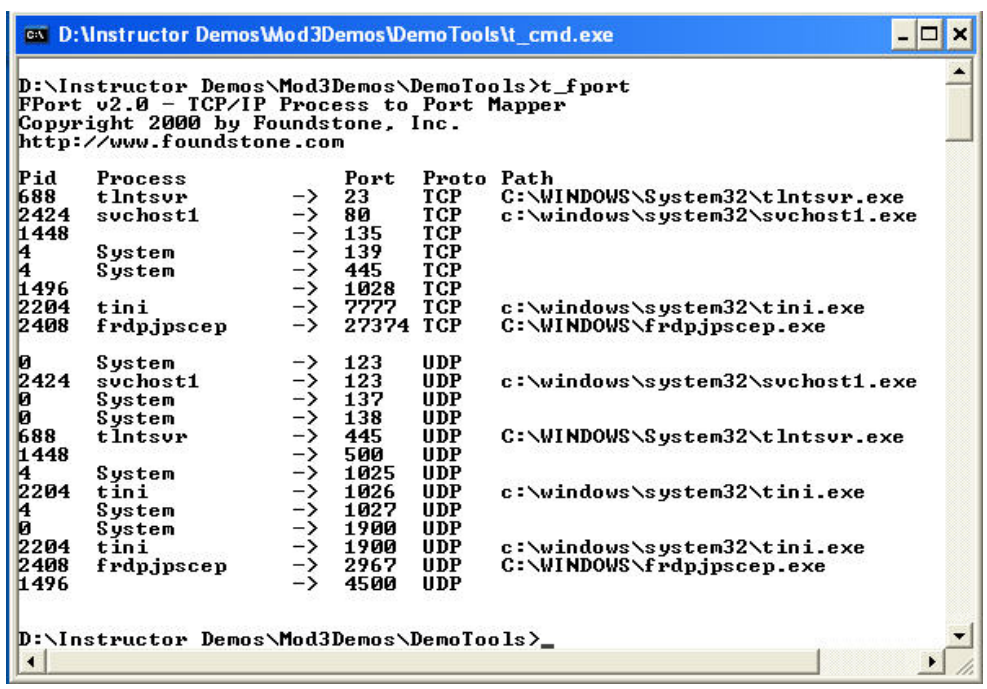

Figure 11: Displaying Open Ports Using fport 


\subsubsection{2 netstat (Linux)}

The Linux netstat command can be used to display all TCP/IP and UDP ports that are open in relation to a running process.

Using netstat with the -tap command line arguments will display all running processes that have a TCP/IP port open, the PID of the process, the port number assignment, the foreign address if connected, and the state of the port (Figure 12).

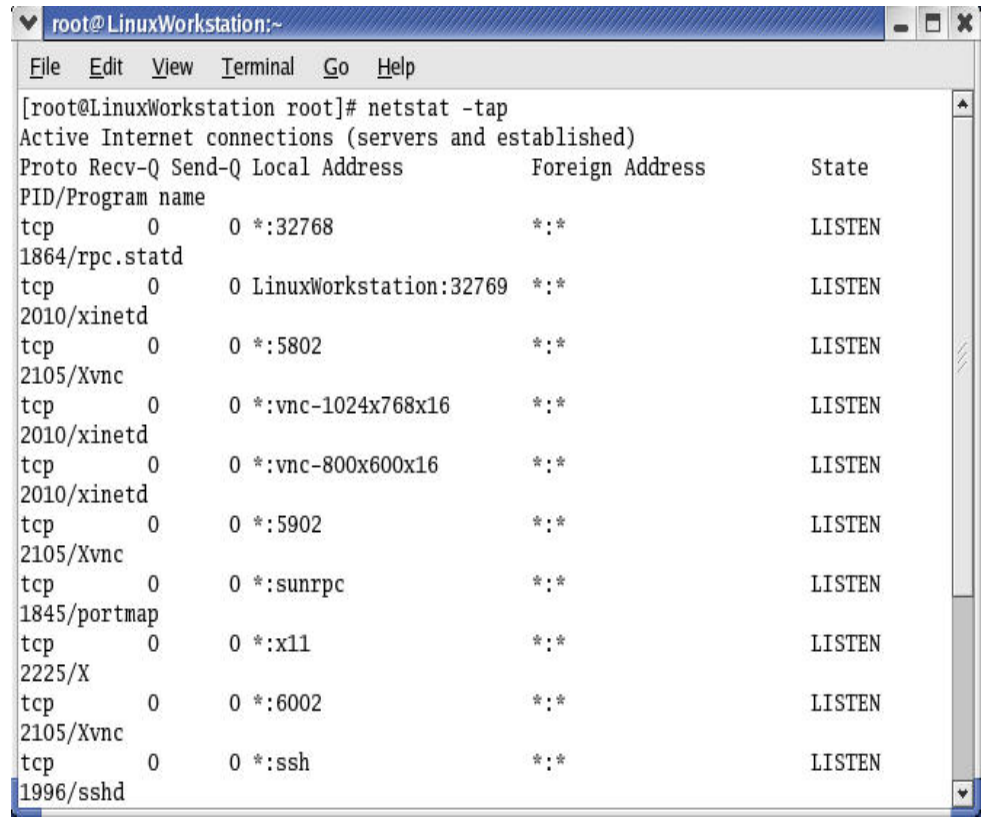

Figure 12: Displaying Open Ports Using netstat 


\section{Potential Rogue Processes}

\begin{tabular}{|l|l|l|l|}
\hline Filename & Port & PID & Location \\
\hline cuoikqkxvs.exe & 27374 & 2288 & C:IWINDOWS \\
\hline svchost1.exe & 80 & 312 & C:I..ISystem32 \\
\hline notepad.exe:alds.exe & 7777 & 988 & C:I..ISystem32 \\
\hline spoolsv.exe & & & \\
\hline
\end{tabular}

\subsubsection{Open Files}

Open files associated with an executing process should not be overlooked. Often, rogue processes such as a key logger or network sniffer will have an associated open file to capture their collected information. One quick way to determine whether a potential rogue process has any current files open is to use the handle.exe utility for Windows and the native Isof command for Linux.

\subsubsection{1 handle (Windows)}

The handle.exe utility displays information about open handles for processes running on the system. You can use it to view the programs that have a file open or to view the object types and names of all the handles of a program.

The screenshot in Figure 13 demonstrates how we can use the handle.exe utility to look at all open handles for the potential rogue process svchost1.

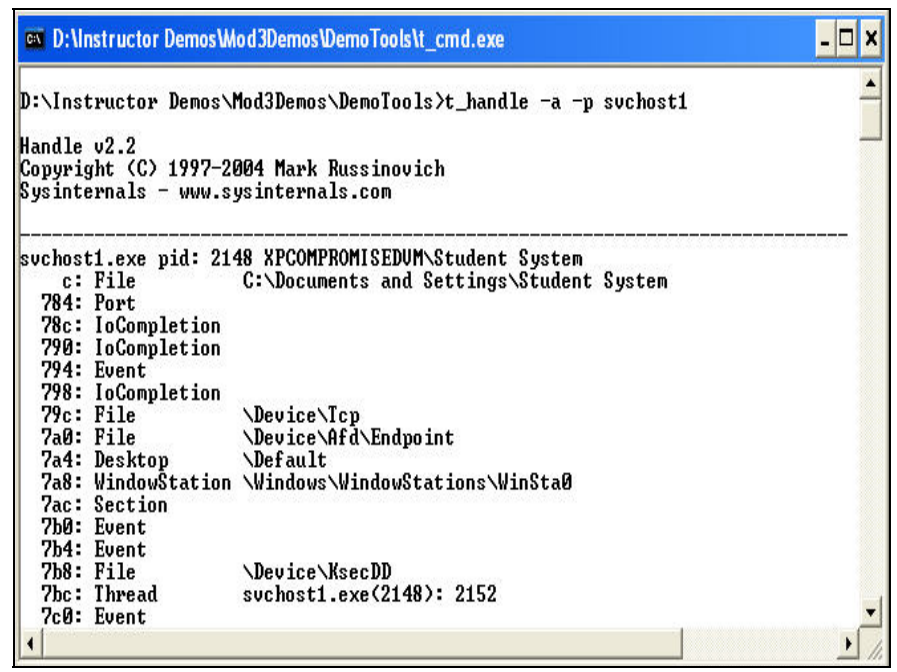

Figure 13: Viewing Handles Using handle 


\subsection{Isof (Linux)}

The native lsof command without any command line arguments will display all open files belonging to all currently running processes. The three screenshots (Figure 14, Figure 15, and Figure 16) demonstrate lsof's command versatility.

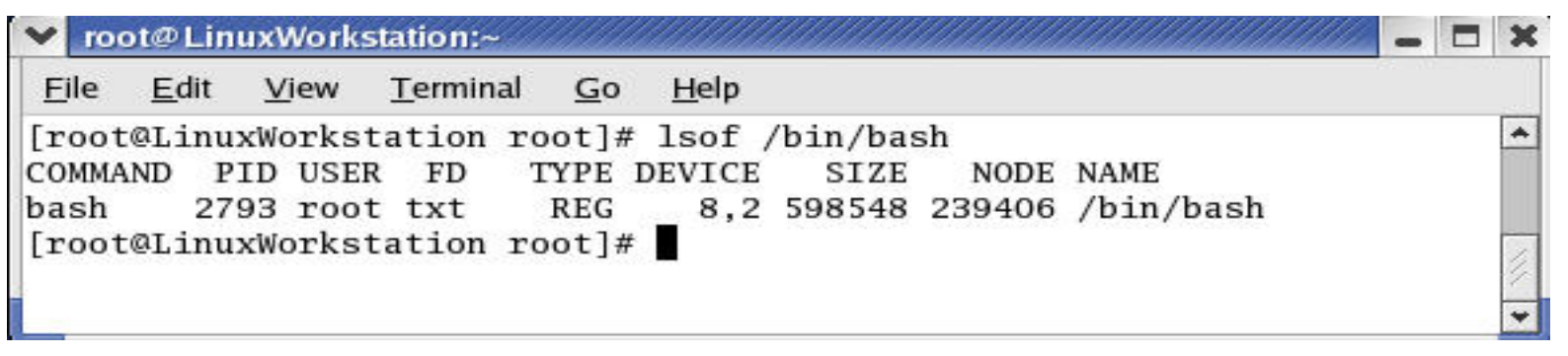

Figure 14: Displaying Which Process Has Port 6002 Open

\section{rooteo LinuxWorkstation:}

File EEdit View Ierminal Go Help

[root@LinuxWorkstation root]\# lsof $-i: 6002$

COMMAND PID USER FD TYPE DEVICE SIZE NODE NAME

Xvnc 2105 root Ou IPv4 $2600 \quad$ TCP $*: 6002$ (LISTEN)

[root@Linuxworkstation root]\#

Figure 15: Displaying Who Has the Bash Shell Open

\begin{tabular}{|c|c|c|c|c|c|c|c|c|c|}
\hline \multicolumn{6}{|c|}{$\checkmark$ root@LinuxWorkstation: } & & & \multirow[t]{2}{*}{ - $\square$} & \multirow[t]{2}{*}{$\mathbf{x}$} \\
\hline Edit & View & Terminal & $\underline{\text { Go }}$ & & & & & & \\
\hline \multicolumn{10}{|c|}{ [root@LinuxWorkstation root]\# lsof -u root | more } \\
\hline COMMAND & PID & USER & TYPE & DEVICE & SIZE & NODE & NAME & & \\
\hline init & 1 & root cwd & DIR & 8,2 & 4096 & & / & & 1 \\
\hline init & & root rtd & DIR & 8,2 & 4096 & & 1 & & \\
\hline init & 1 & root txt & REG & 8,2 & 27036 & 319298 & /sbin & / in & - \\
\hline
\end{tabular}

Figure 16: Displaying All the Currently Open Files by the User Root

\subsubsection{Base Priority}

When a process is initially executed for both Windows and Linux it is assigned a base priority value. That value determines what priority it has over other processes in regard to the computer resources it is assigned and consumes, such as memory and CPU time. When looking at a potential rogue or runaway process you may want to check the assigned priority value. 


\subsubsection{1 pslist (Windows)}

Using Sysinternal's pslist.exe utility we can enumerate the priority levels for each current running process by looking at the "Pri" column. The screenshot in Figure 17 demonstrates how to display priority values for running processes.

\subsubsection{2 top (Linux)}

Using the native Linux top command we can enumerate the priority levels for each current running process. Linux processes will generally have a priority value between -20 and 19 , where the value of -20 is the highest and 19 is the lowest priority value (Figure 18).

Since the top command only displays the top current processes, if we need to enumerate the process priority value for a particular process we can use the top command with the command line arguments $-p$

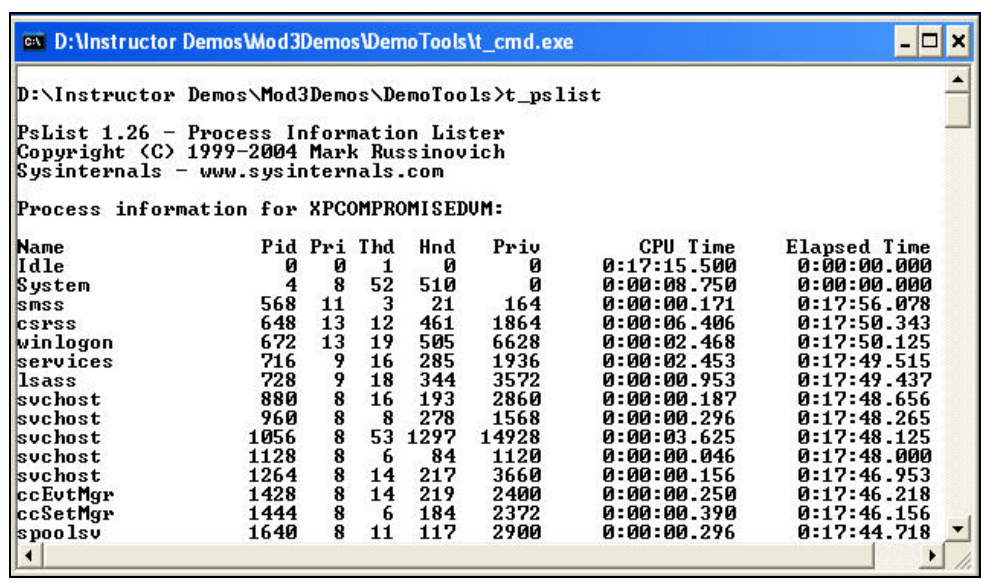

Figure 17: Listing Priority Levels Using pslist

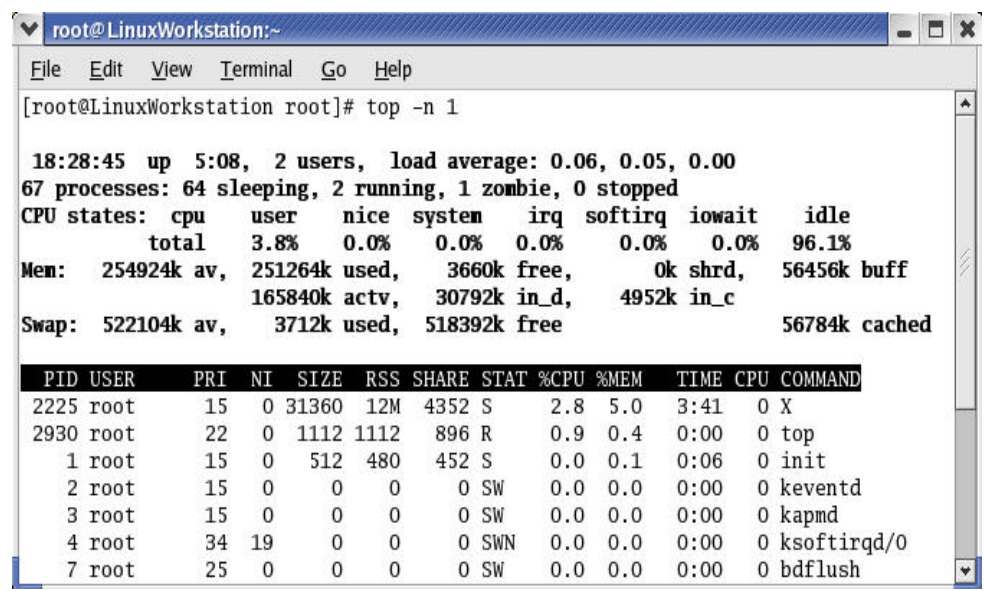

Figure 18: Listing Priority Levels Using top PID - $\mathrm{n}$ 1, as shown in Figure 19.

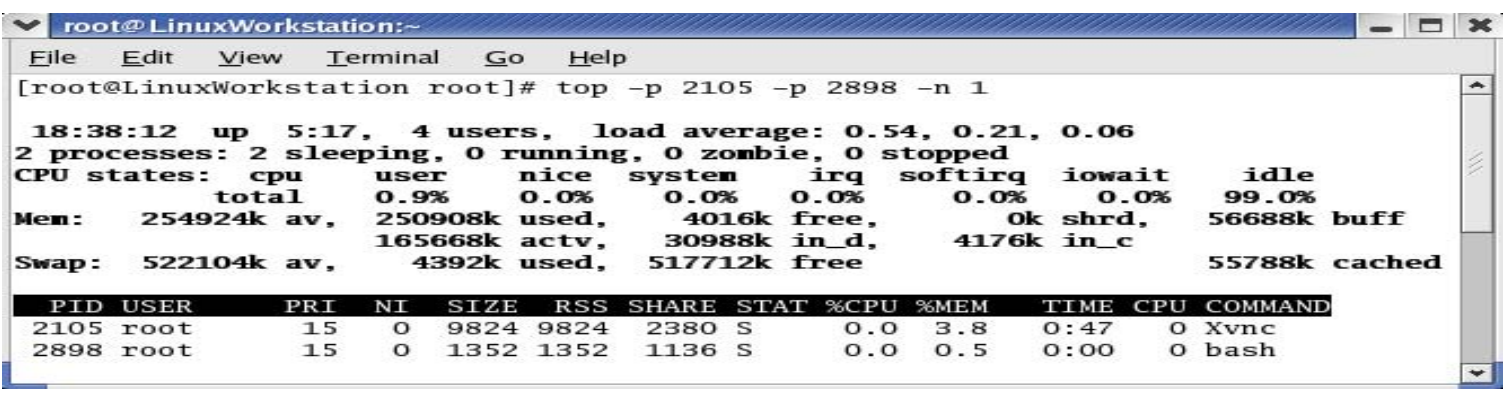

Figure 19: Displaying the Priority Level for a Specific Process 


\subsubsection{Process Times and Terminated Processes}

\subsection{Process Start Time}

The process start time is the point in time when the process started executing. An interesting characteristic of rogue processes is that they generally will have a start time that is a few seconds or few minutes later than all other legitimate running processes. To discover processes that may have started later in time after the boot cycle you can use the psuptime.exe and pslist.exe command line tools. These tools will tell you when a process first started.

\section{psuptime (Windows)}

To calculate the initial start time of a process, we first have to collect the uptime or how long the system has been running. We can do this using the psuptime.exe utility.

Once we have the uptime of the system, the next step is to enumerate all of the elapsed times for the current set of run-

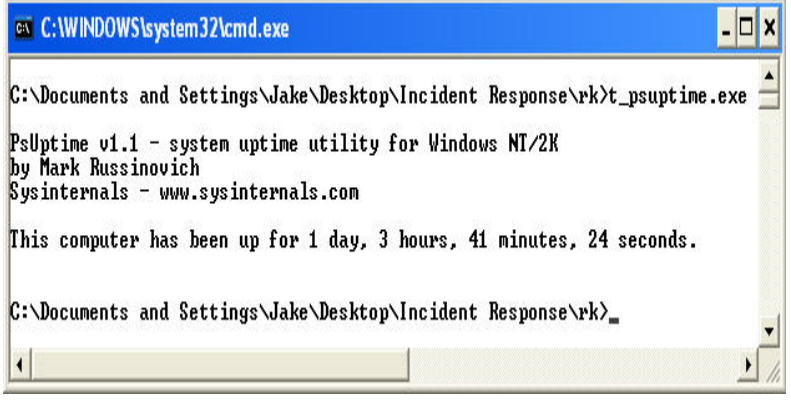

Figure 20: Checking Uptime Using psuptime ning processes using pslist.exe.

\subsection{Process Elapsed Time}

\section{pslist (Windows)}

Once we have the uptime of the system and the elapsed time for a particular process we can simply subtract the (Uptime - Elapsed time) to calculate Start Time for any given process.

For example, the Uptime of the system was $=27 \mathrm{~h}: 41 \mathrm{~m}: 24 \mathrm{~s}$ and the Elapsed Time of the Svchost.exe process was = 3h:34s:41s; therefore, the Start Time for the Svchost.exe process was 24h:6m:43s (12:06:43 a.m.).

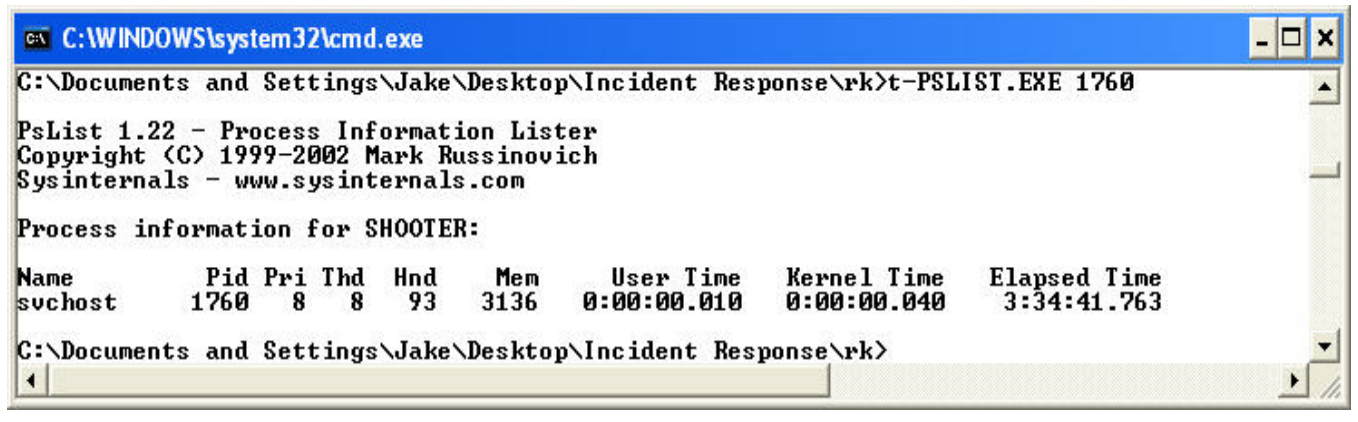

Figure 21: Checking Elapsed Time for a Process Using pslist 
Note that, by just looking at the pslist.exe output, more specifically the elapsed times, you can determine which processes started after the boot process.

\subsection{Terminated Processes}

Suspended or prematurely terminated processes can be indicators of abnormal system behavior. Often in computer security situations, a critical system process will be suspended or terminated. For example, an antivirus or other critical process may be terminated in an attempt to prevent the host system's security mechanisms from checking for running malware on the system. Therefore, terminated processes should be collected and identified.

One method to check for terminated processes in Windows, assuming proper auditing is enabled, is reviewing the event logs.

The screenshot in Figure 22 displays a system event log using the Windows Event Viewer. A terminated process in Windows exhibits an Event ID of 7034.

Knowing what Event ID to search for, we can use a command line utility called psloglist.exe developed by Sysinternals to collect and parse through the entire system event logs looking for only event logs that exhibit the Event ID of 7034.

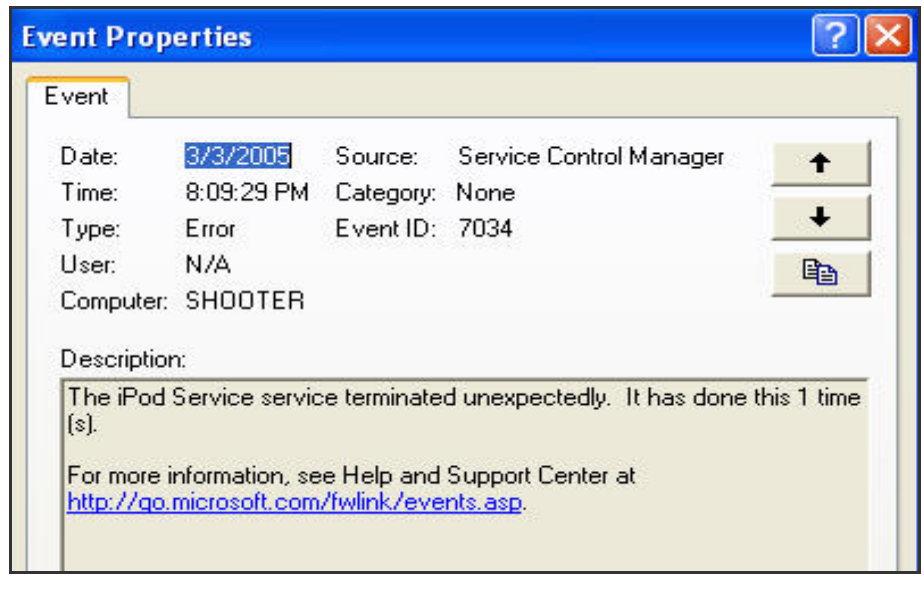

Figure 22: Windows Event Log

Figure 23 demonstrates how to use the psloglist.exe utility with the $-i$ command line argument to search for all event logs that have the Event ID of 7034.

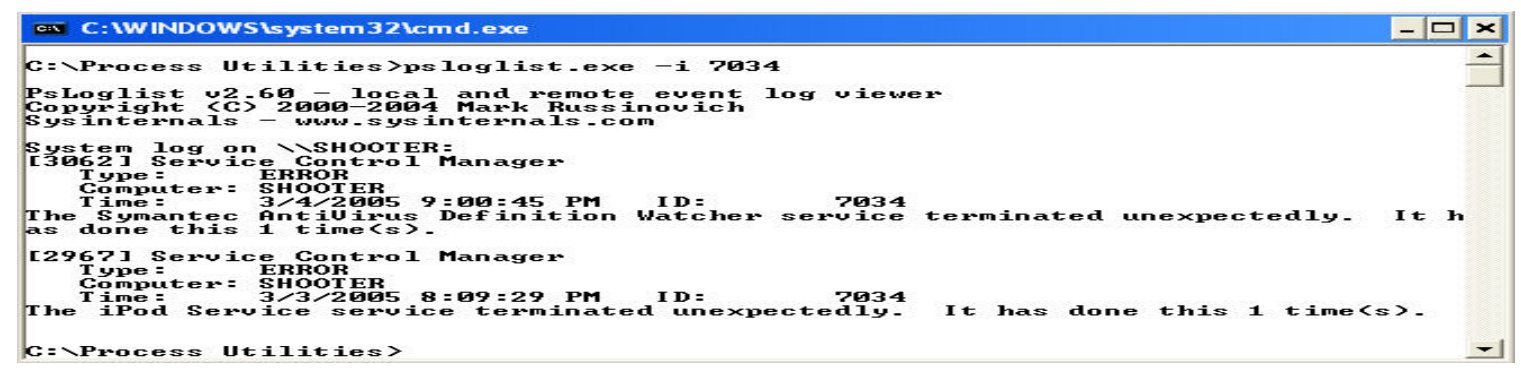

Figure 23: psloglist Command 


\subsection{Process Terminated Time}

As stated, we can determine which processes have been terminated by using Sysinternals' psloglist.exe. Using the same approach, we can see that the output of the collected event log clearly states at what time the process was terminated.

\subsubsection{Location of Process Image}

The location of the process image can give you further insight into whether the process is a legitimate or rogue process. For example, if a running process's image is located in the Startup folder or another anomalous file location, there is a good chance that the currently running process is not legitimate. We can quickly identify the location of a process image by using the following utilities for Windows and Linux.

\subsection{ListDLLs (Windows)}

Using Sysinternals' ListDLLs utility we can determine the command line used to execute the process and the location of the process image.

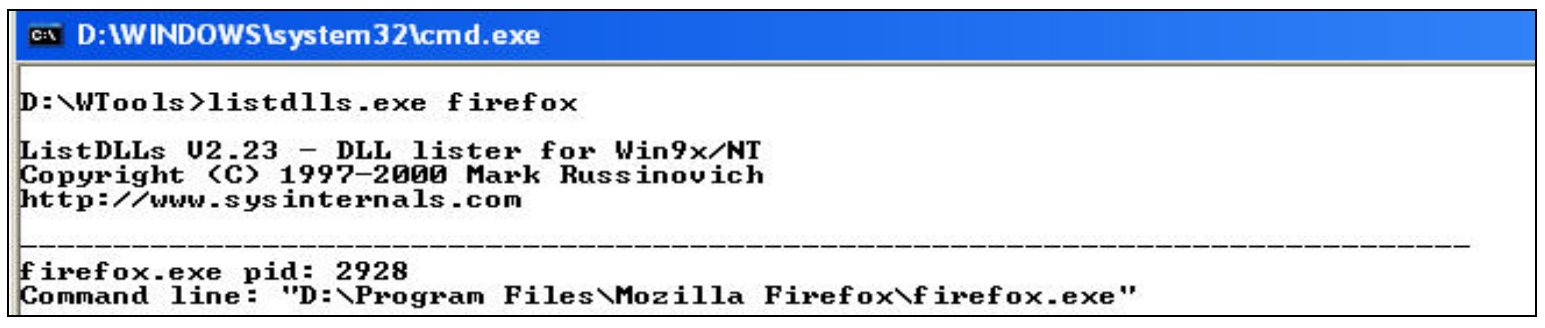

Figure 24: Locating a Process Image Using ListDLLs

\subsubsection{2 ps and Isof (Linux)}

Using the native Linux ps command with the -aux command line arguments, we can determine the command line used to execute the process and the location of the process image.

\begin{tabular}{|c|c|c|c|c|c|c|c|c|c|c|c|c|}
\hline \multicolumn{8}{|c|}{$\checkmark$ roote LinuxWorkstation: ? } & \multirow[b]{3}{*}{ s } & \multirow[b]{3}{*}{$13: 40$} & \multirow[b]{3}{*}{$0: 05$} & \multirow[t]{2}{*}{ - $\square$} & \multirow[t]{2}{*}{$x$} \\
\hline File & Edit & View & \multicolumn{2}{|c|}{ Ierminal } & Go & Help & & & & & & \\
\hline root & & 2362 & 0.0 & 2.2 & 16044 & 5664 & ? & & & & magicdev --sm-client-id default & - \\
\hline root & & 2368 & 0.0 & 1.5 & 11320 & 3944 & ? & $\mathrm{s}$ & $13: 40$ & $0: 00$ & pam-panel-icon--sm-client-id d & \\
\hline root & & 2371 & 0.3 & 6.3 & 27876 & 16140 & & SN & $13: 40$ & $1: 05$ & /usr/bin/python /usr/bin/rhn-ap & \\
\hline root & & 2373 & 0.0 & 2.5 & 16592 & 6548 & ? & $\mathrm{s}$ & $13: 40$ & $0: 00$ & /usr/libexec/notification-area- & \\
\hline root & & 2375 & 0.0 & 0.2 & 1564 & 516 & $?$ & s & $13: 40$ & $0: 00$ & /sbin/pam_timestamp_check -d ro & \\
\hline root & & 2419 & 0.0 & $2 . \overline{9}$ & 13432 & 7436 & ? & s & $13: 42$ & $0: 07$ & metacity --sm-save-file 1111776 & \\
\hline root & & 2896 & 0.3 & 4.6 & 22664 & 11928 & $3 ?$ & $\mathrm{R}$ & $18: 28$ & $0: 10$ & gnome-terminal & \\
\hline root & & 2897 & 0.0 & 0.2 & 1996 & 612 & $?$ & s & $18: 28$ & $0: 00$ & gnome-pty-helper & \\
\hline root & & 2898 & 0.0 & 0.5 & 4280 & 1352 & pts/o & s & $18: 28$ & $0: 00$ & bash & \\
\hline root & & 2935 & 0.0 & 0.5 & 4276 & 1352 & pts $/ 1$ & $\mathrm{~s}$ & $18: 36$ & $0: 00$ & bash & 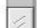 \\
\hline root & & 3078 & 0.0 & 0.3 & 2744 & 768 & pts $/ 1$ & $\mathrm{R}$ & $19: 18$ & $0: 00$ & ps -aux & \\
\hline [root & alint & 1xworks & stati & on $\mathrm{rc}$ & t] \# & & & & & & & - \\
\hline
\end{tabular}

\section{Figure 25: Locating a Process Image Using ps}

Using the lsof command with the -p PID command line argument, we can enumerate the location of the process image defined by process ID or PID and also any other files open by the defined process. 


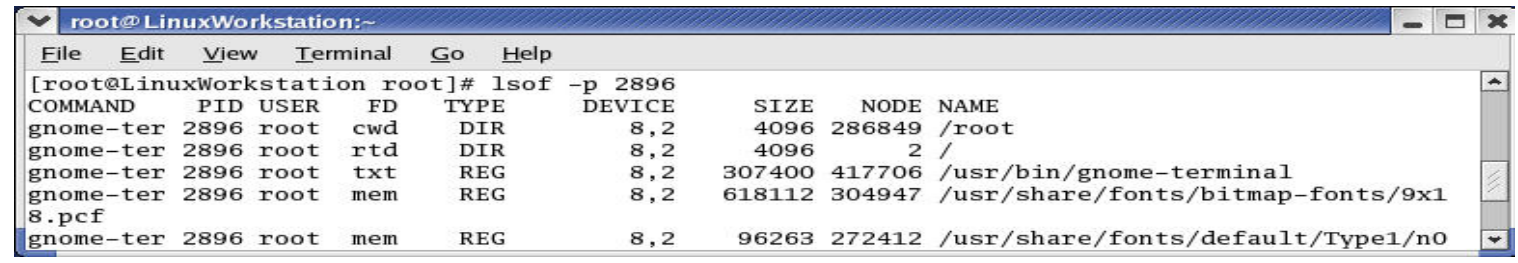

Figure 26: Locating a Process Image by PID

\subsubsection{Survivable Processes}

Survivable processes can be defined as processes that will re-execute after the machine has been shut down and then restarted. Often when malware infects a machine or if an attacker compromises the system, the stored malicious code will be located in startup locations on the system. Also, registry keys and values may have been modified so that the malware processes will be started up again upon system reboot. Question certain file locations in the system.

- $\quad$ startup folders, added registry key values and scripts

Check to see if unauthorized applications are starting upon reboot. There are a number of different methods an intruder can use to start a backdoor program, so be sure to check the startup folders, startup scripts, and even registry key values.

- invalid services

Check for invalid services. Some backdoor programs will install themselves as services so they are started when the system boots up.

- scheduled tasks

Check for scheduled tasks and Crontab files.

\subsection{Startup Folders, Registry Keys and Values (Windows)}

Here are some common Windows startup locations/folders and registry keys to consider.

- Check all items in the C:|Documents and Settings $\backslash$ All Users|Start Menu\Programs IStartup folder. Note that there are two startup folders, one for the local user and one for all other users on the system. When a user logs on, all of the applications in both the local user's and in the All Users startup folders are started. Because of this, it is important to check both of the startup folders.

- Check the registry for added keys and key values. The most common locations for applications to start through the registry are the following:

- HKEY_LOCAL_MACHINEISystem|CurrentControlSet|Control|Session Manager $\backslash$ KnOWnDLLS

- HKEY_LOCAL_MACHINE|System|ControlSet001|Control|Session Manager $\backslash$ KnOWnDLLS

- HKEY_LOCAL_MACHINE|Software|Microsoft|Windows|Current Version|Run

- HKEY_LOCAL_MACHINE|Software|Microsoft|Windows|Current Version|RunOnce 
- HKEY_LOCAL_MACHINE|Software $\mid$ Microsoft $\mid$ Windows $\mid$ Current VerSion\RunOnceEX

- HKEY_LOCAL_MACHINE|Software|Microsoft|Windows|CurrentVersion|RunServices

- HKEY_LOCAL_MACHINEISoftwarelMicrosoft $\mid$ Windows NT $\mid$ CurrentVersion $\mid$ Windows ("run=" line)

- HKEY_CURRENT_USERISoftware|Microsoft|Windows|Current Version|Run

- HKEY_CURRENT_USER|Software|Microsoft|Windows|Current Version|RunOnce

- HKEY_CURRENT_USERISoftware|Microsoft|Windows|Current Version|RunOnceEx

- HKEY_CURRENT_USERISoftware|Microsoft|Windows|CurrentVersion|RunServices

- HKEY_CURRENT_USER|Software|Microsoft|Windows NT|CurrentVersion|Windows ("run=" value)

\section{autorunsc (Windows)}

The autorunsc.exe utility developed by Sysinternals allows you to collect all of the following information regarding survivable processes and services:

- $\quad$ startup applications and their location

- registry key values

- $\quad$ startup services and their location

Figure 27 is a demonstration of using the autorunsc.exe utility to collect information about processes that will be started upon reboot. For example, look at the executables located in the Startup folder.

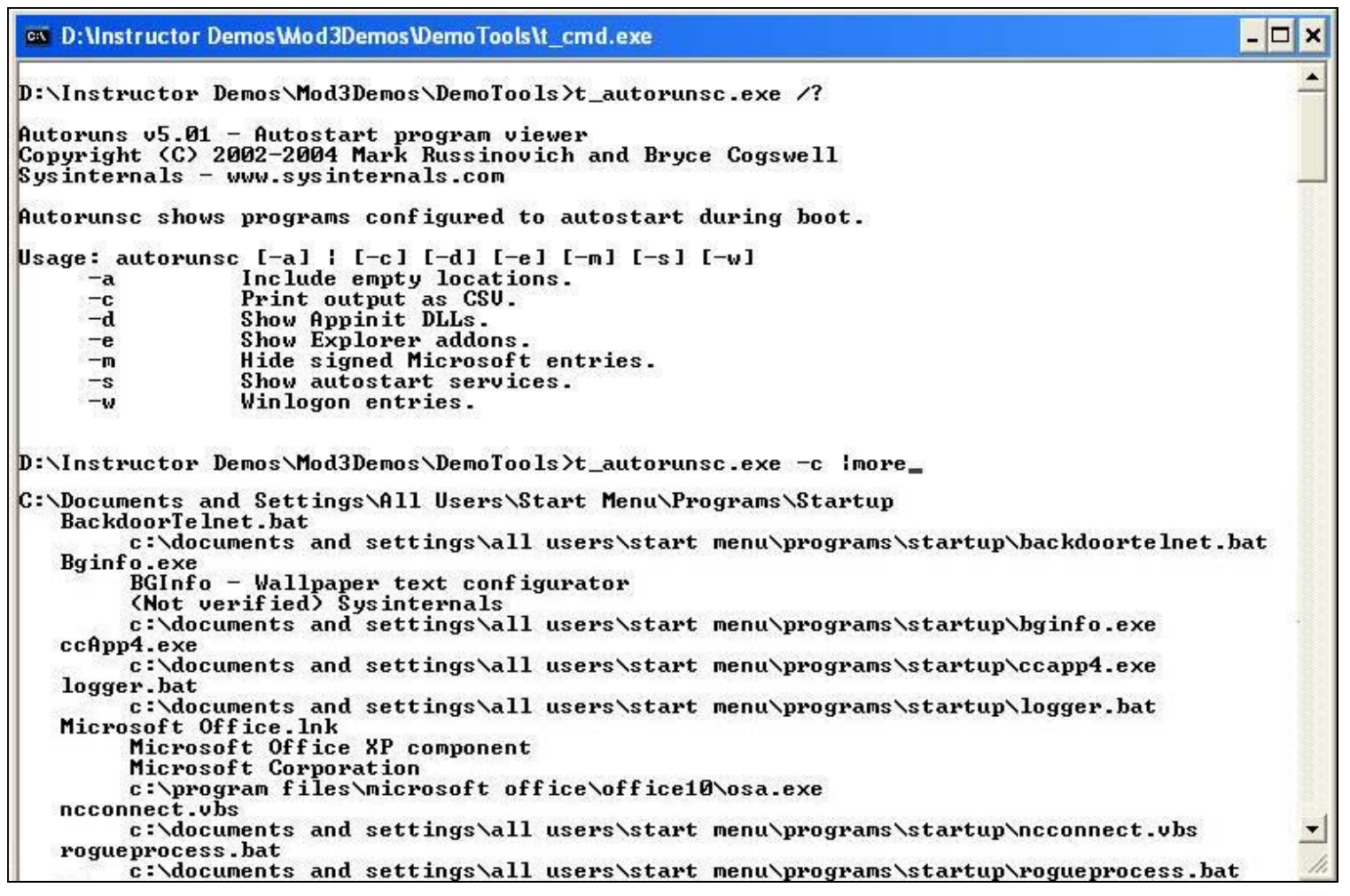

Figure 27: autorunsc.exe Command 


\section{Startup Locations and Scripts (Linux)}

For a Linux system, certain files should be examined to determine whether there are malicious scripts within these files. Often an attacker will place a shell script in one of the following files so that it gets executed every time the machine is rebooted. This is not an exhaustive list by any means, but includes common file locations to consider and check.

- $\$$ etc/rc.local

- \$ etc/initab

- $\$$ etc/rc.sysinit

\subsection{Invalid Services}

\section{Checkconfig (Linux)}

The chkconfig -list command displays a list of services that will be run at the five different runlevels. This information may help you identify a rogue or malware application that is set to run as a service and at one of the five runlevels.

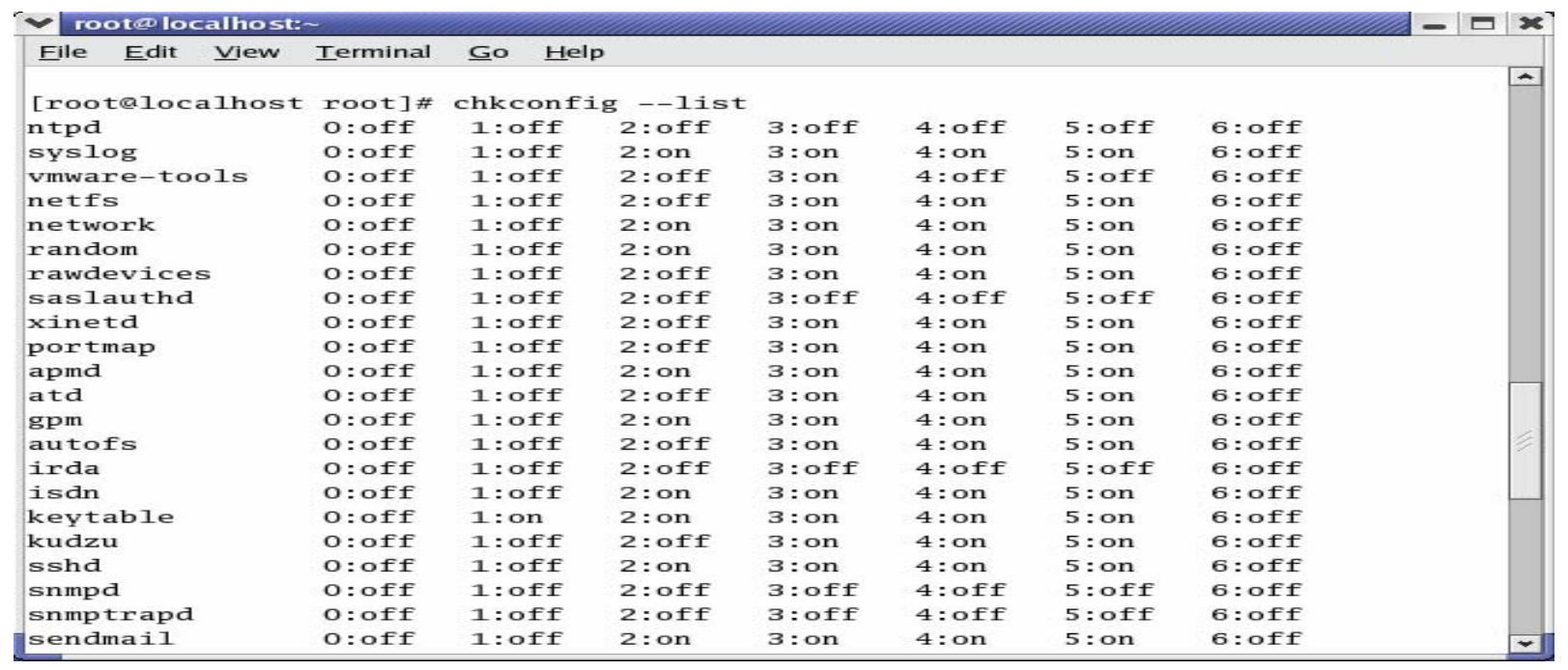

Figure 28: The chkconfig -list Command

Corresponding to the five levels,

chkconfig has five distinct functions: adding new services for management, removing services from management, listing the current startup information for services, changing the startup information for services, and checking the startup state of a particular service.

When chkconfig is run without any options, it displays usage information. If only a service name is given, it checks to see if the service is configured to be started in the current runlevel. If it is, chkconfig returns true; otherwise it returns false. 
The --level option may be used to have chkconfig query an alternative runlevel rather than the current one [Haas 04].

\subsection{Scheduled Tasks}

\section{at (Windows)}

For collecting information about scheduled tasks on a Windows machine, use the native at.exe command. The at.exe command will display currently scheduled tasks. Scheduled tasks should not be overlooked because an attacker could essentially schedule a certain file, executable, or script to be run on a certain day or time of day that could cause malicious system behavior.

\section{Cron Logs (Linux)}

In addition to startup services, you should collect the currently scheduled tasks. Attackers often schedule a malicious file to execute periodically so that the malware remains existent. The cron feature allows system administrators to schedule programs for future execution. All executed cron jobs are logged, usually in the /var/cron/log or in the default logging directory in a file called cron.

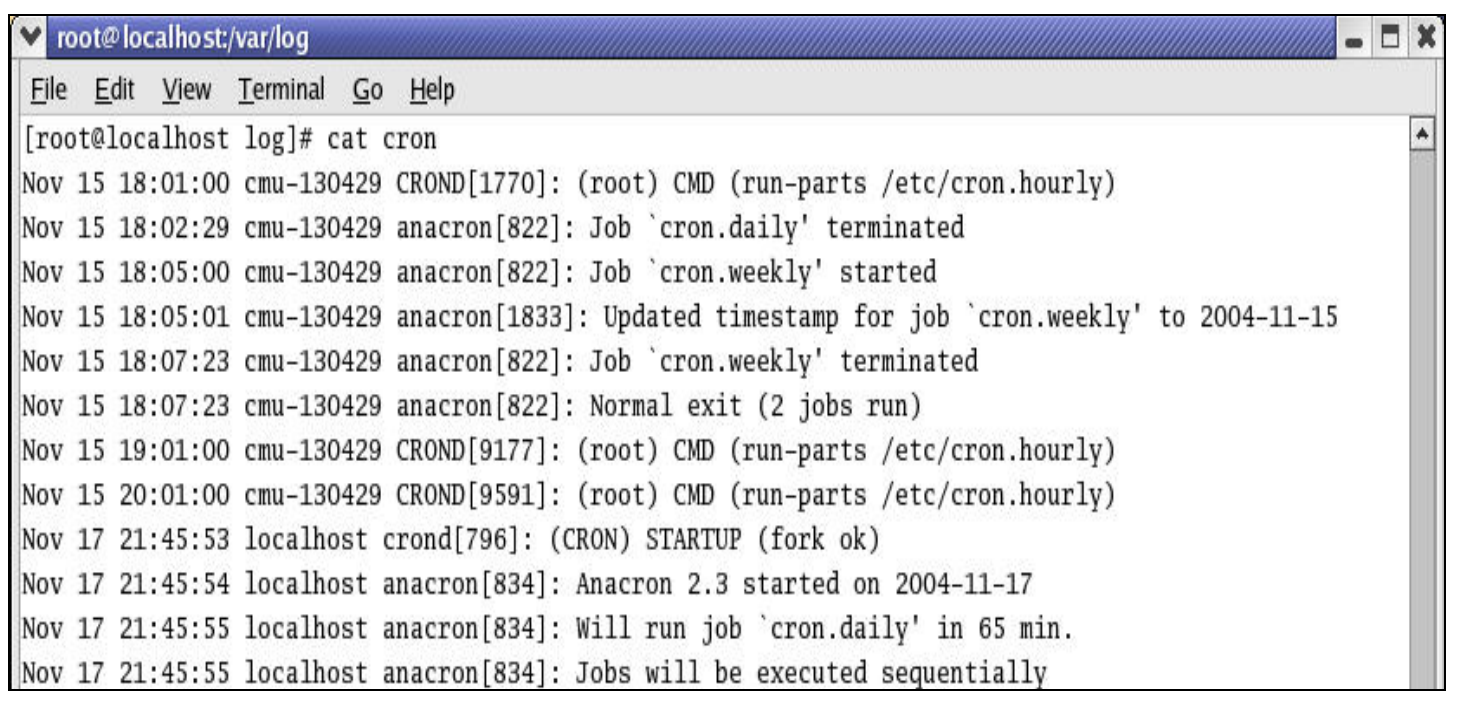

\section{Figure 29: A Cron Log}

\section{Crontab (Linux)}

Using the crontab command, we can collect the currently created cron jobs for each user of the system. 


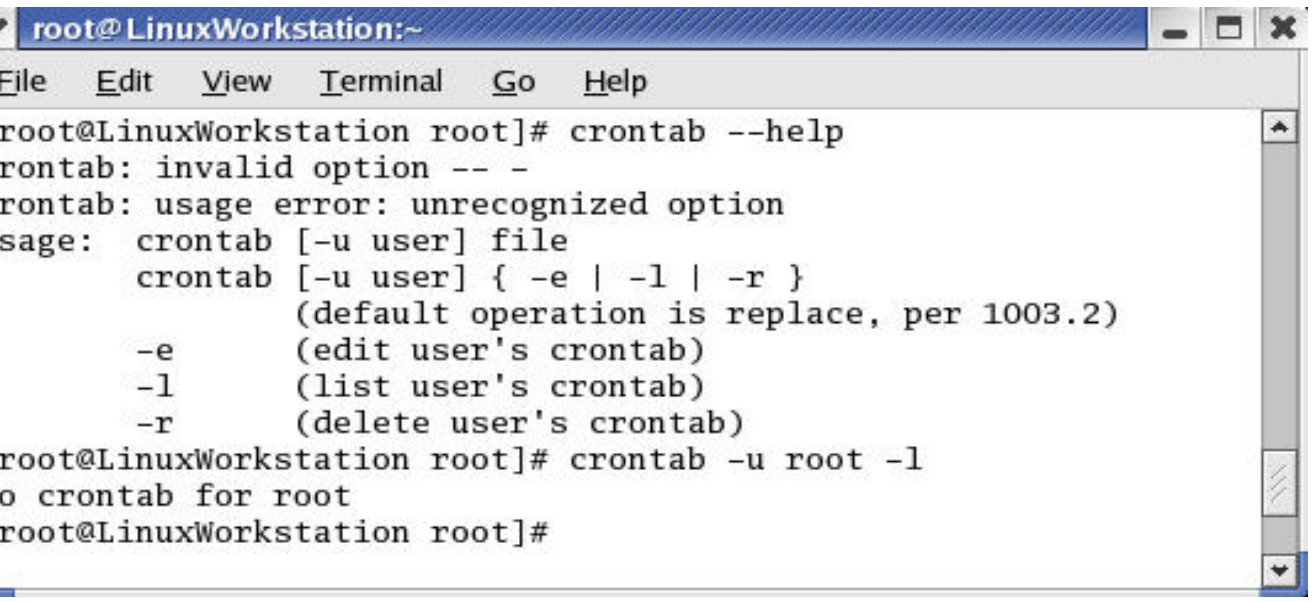

Figure 30: The Crontab Command 


\section{Process Forensic Tasks}

1. Check and verify a process's loaded DLLS

(Dynamically Linked Libraries)

2. Check and verify a process image

- Hash and compare the spoolsv.exe binary

3. Process string search and analysis on

- svchost1.exe

- spoolsv.exe

- notepad.exe:alds.exe

Utilities used:

- strings.exe

- grep.exe

- md5sum.exe or ma5deep.exe

- sfind.exe

\subsubsection{Process Forensic Tasks}

In the following paragraphs we are going to step through a few process forensics tasks that involve diving deeper into investigating a running process's binary. In the previous sections we demonstrated how to forensically collect eight process characteristics using either native commands or third-party utilities. Now we are going to step through three forensic tasks that will be of importance to a first responder. By performing these three forensic tasks, a first responder can gain further insight into a process's binary function and use.

\subsection{Check and Verify a Process's Loaded DLLs}

Often running processes will be utilizing one or more dynamically linked libraries (DLLs) for Windows or shared libraries in Linux. Dynamically linked and shared libraries are created so they can be used by many different applications and processes. Having these shared libraries drastically reduces the size of the actual executable or process binary and improves system efficiency.

The main reason why DLLs used by a process should not be overlooked is that malware has been known to replace critical system DLLs with malicious ones or add new malicious DLLs to the system in an attempt to cause undesired system behavior once executed. The majority of root kits use DLL injection to infect systems. For these reasons, we will demonstrate how to check and verify the loaded DLLs for a particular process against a known safe set. 
To verify a process's required DLLs involves three steps: (1) identify a process to check, (2) identify the DLLs used by the process, and (3) check to see if the DLLs have been corrupted by hashing them and comparing them against a known safe set.

\section{Step 1: Identify the Process}

Identify the potential rogue process by using a process enumeration utility such as pslist.exe. The screenshot in Figure 31 displays the highlighted process used for this forensic task of checking and verifying its loaded DLLs.

\begin{tabular}{|c|c|c|c|c|c|c|c|c|c|c|}
\hline \multicolumn{9}{|c|}{ c:i C:IWINDOWStsystem32lcmd.exe } & \multicolumn{2}{|c|}{$-\square \times$} \\
\hline $\begin{array}{l}\text { mozilla } \\
\text { suchost } \\
\text { POWERPNT } \\
\text { WINWORD } \\
\text { wmplayer } \\
\text { cmd } \\
\text { SnagIt32 } \\
\text { TSCHelp } \\
\text { t-PSLIST }\end{array}$ & $\begin{array}{r}2248 \\
780 \\
928 \\
2376 \\
3984 \\
2596 \\
1376 \\
2372 \\
528\end{array}$ & $\begin{array}{r}8 \\
8 \\
8 \\
8 \\
8 \\
8 \\
8 \\
8 \\
13\end{array}$ & $\begin{array}{r}10 \\
8 \\
4 \\
10 \\
37 \\
1 \\
4 \\
1 \\
2\end{array}$ & $\begin{array}{r}240 \\
93 \\
266 \\
574 \\
777 \\
31 \\
226 \\
29 \\
87\end{array}$ & $\begin{array}{r}25936 \\
3136 \\
1604 \\
51412 \\
29532 \\
1268 \\
19124 \\
2288 \\
1664\end{array}$ & 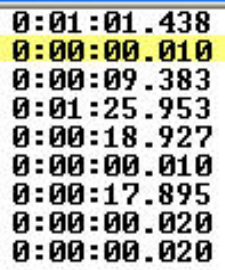 & 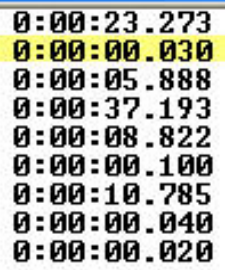 & $\begin{array}{r}14: 36: 48.302 \\
5: 49: 10.722 \\
1: 40: 49.098 \\
1: 40: 47.696 \\
1: 35: 47.474 \\
0: 57: 33.385 \\
0: 48: 98.753 \\
0: 48: 07.572 \\
0: 00: 00.390\end{array}$ & & $\Delta$ \\
\hline \multicolumn{9}{|c|}{$c: \backslash$ Documents and Settings $\backslash$ Jake $\backslash$ Desktop $\backslash$ Incident Response $\backslash r k>=$} & & 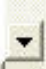 \\
\hline \multicolumn{9}{|l|}{4} & 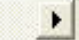 & \\
\hline
\end{tabular}

Figure 31: The svchost.exe 780 Process

\section{Step 2: Identify the DLLs Used by the Process}

The second step is to identify all required DLLs for the identified process. To identify all DLLs required by the svchost.exe process, use the listdlls.exe utility with the command line argument $-p$ 780. By using the $-p$ command line argument, we are instructing the utility to display loaded DLLs only for the designated process (i.e., PID 780).

Figure 32 displays all of the DLLs required for the svchost.exe or PID 780 process. 


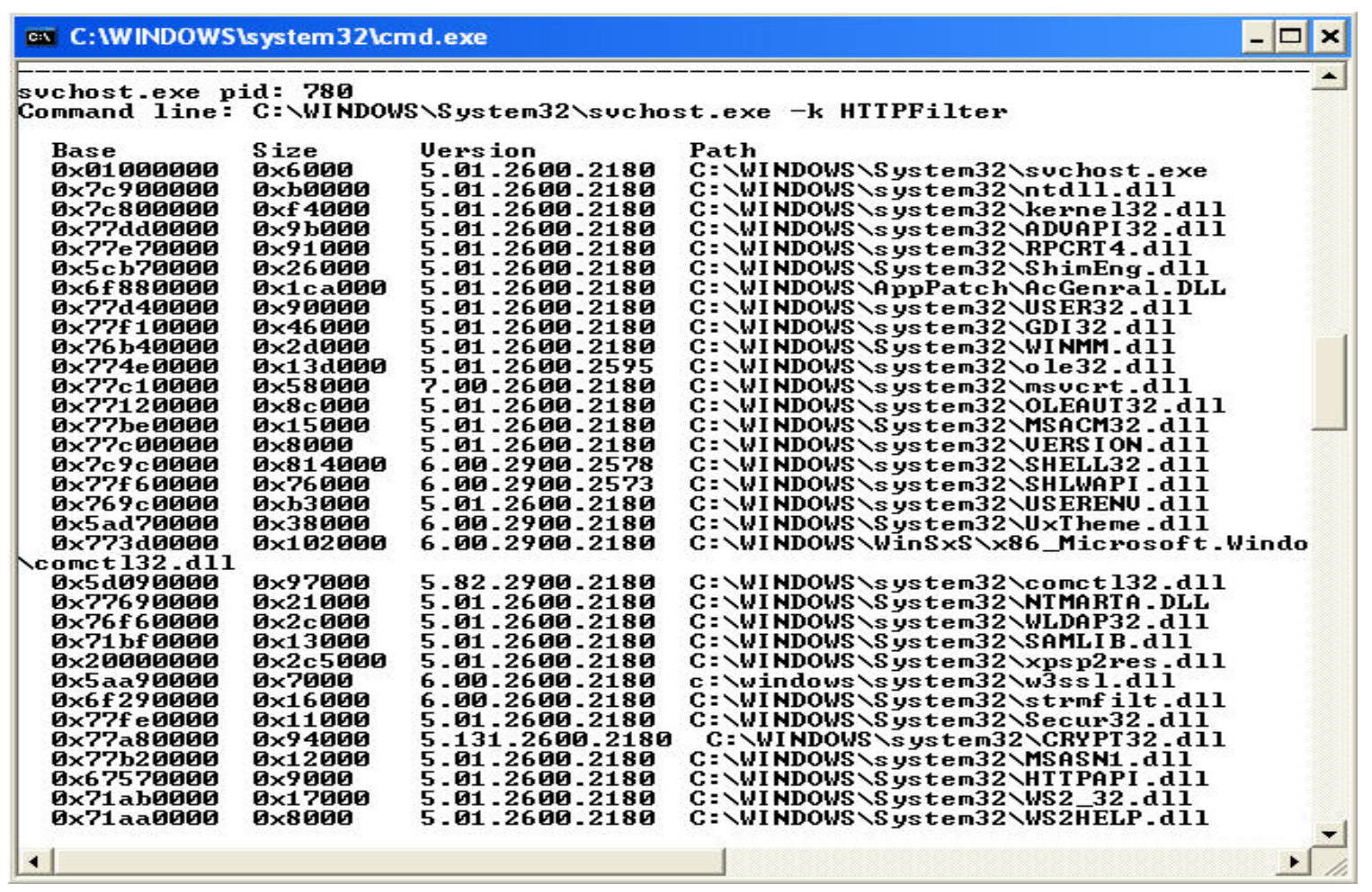

Figure 32: listdlls.exe Output for svchost.exe

\section{Step 3: Hash and Verify Each of the DLLs}

The third step in our quest to check and verify the loaded DLLs for the svchost.exe process is to hash and compare each loaded DLL against a safe set. To hash each one of the DLLs, you can use any cryptographic hash utility. For this demonstration we used the MD5deep.exe utility to perform a recursive hash on all DLL files stored in the C:IWINDOWSISystem32 folder and its subfolders. Later, we will search for the identified DLLs to make sure they hashed correctly.

The primary reason to perform a recursive hash on all files in the System32 folder is that it is a much quicker method of hashing all of the necessary svchost.exe process loaded DLLs, since most of them reside in the System32 folder. Otherwise, we would have to hash each one of the DLLs separately, which there is no easy way to do and which would take a serious amount of time.

The MD5deep.exe hash utility works by first computing cryptographic hashes for all files you designate from the command line and then comparing the computed hashes against a defined file that contains a safe set of hashes. The safe set of hashes that we will be using to compare against is the National Software Research Libraries library of cryptographic hashes.

Figure 33 displays the MD5deep.exe utility's command line options, as well as an example of the command line syntax used to perform a recursive MD5 hash on all files in a designated directory. The recursive hash is performed on the System32 folder and its subdirectories. The utility then compares those computed hashes against a stored set of safe hashes, which are 
stored in the NSRLFile.txt file. The matched hashes are then output to a DLLs.txt text file, which we will search later. Note that when the $-M$ command line argument is used, the utility will flag and output to the DLLs.txt text file only hashes of files that the MD5deep.exe utility matched correctly against the NSRLFile.txt.

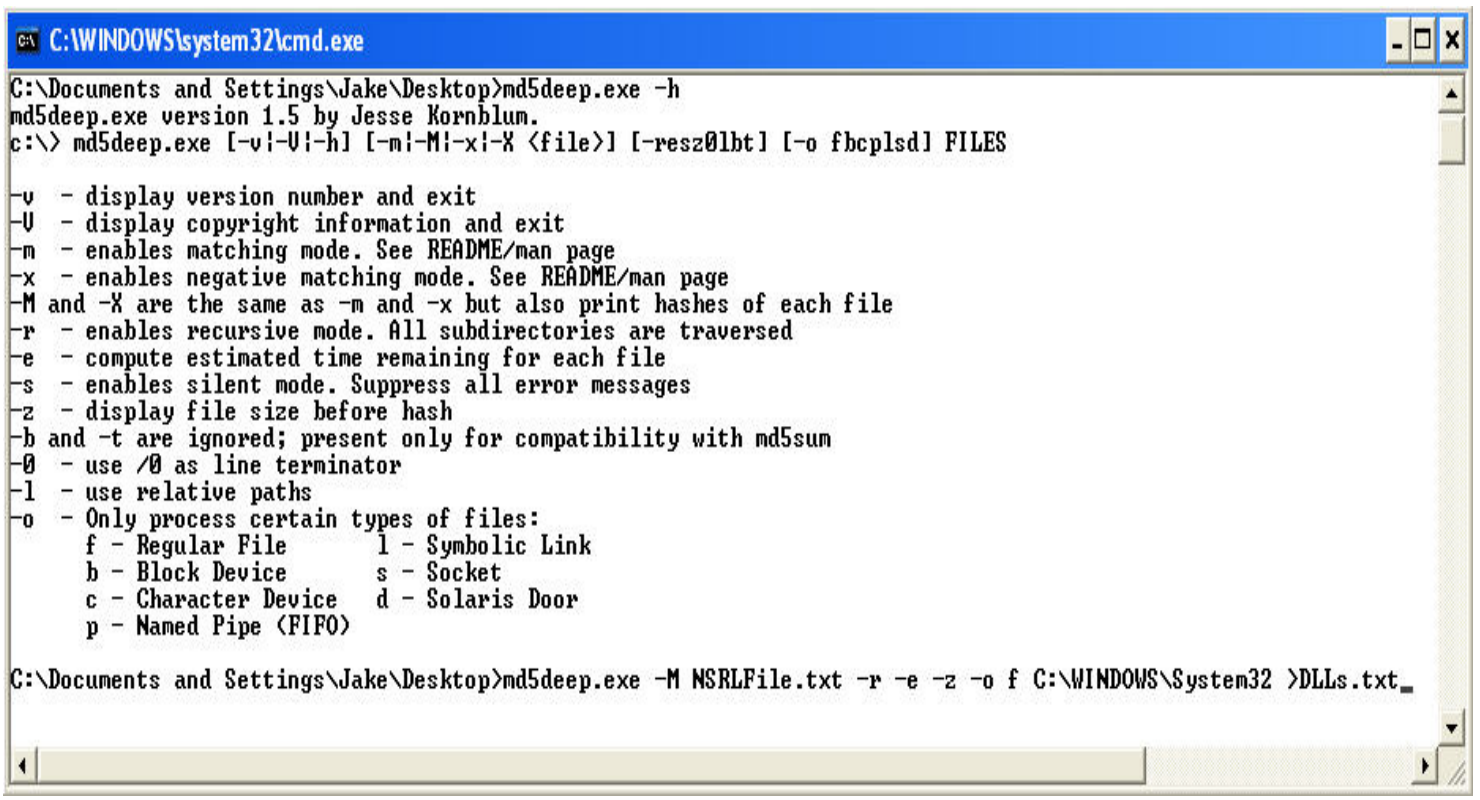

\section{Figure 33: MD5deep Utility}

\subsection{The Search}

To find out which svchost.exe DLLs hashed correctly, we can perform a string search on the DLLs.txt file to look for the DLLs used by the svchost.exe process. The search criteria will be the filenames of the listdlls.exe utility output for the svchost.exe process.

Figure 34 is a screen shot of using the grep.exe utility for performing a string search on the DLLs.txt text file to see if hashes for the required DLLs exist. Note that this is not all of the DLLs that the svchost.exe process required, but for demonstration purposes three DLLs are provided.

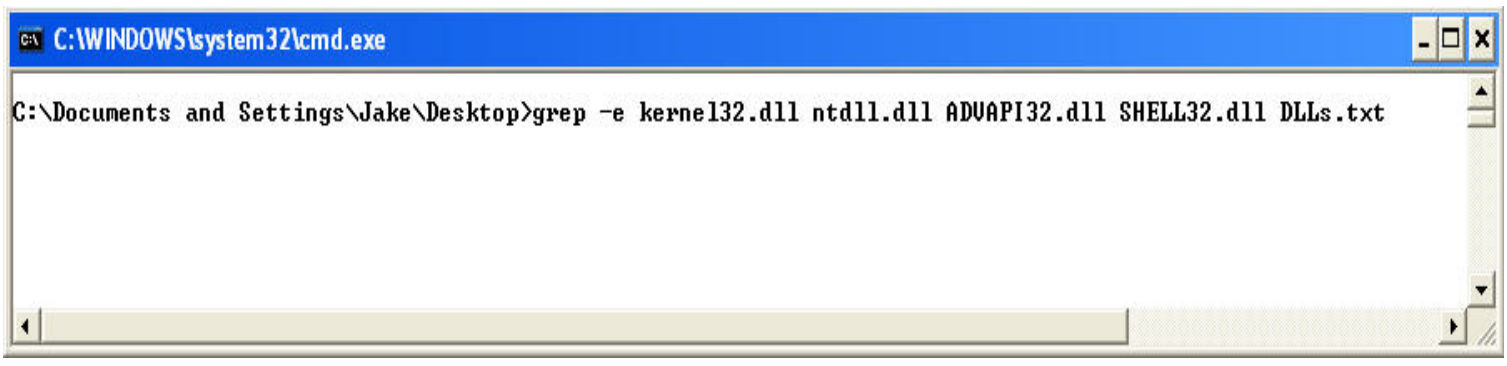

Figure 34: Performing a String Search Using grep 
If everything goes well, the output of your search should be hashes of the files you expected. However, if your search of the DLLs.txt file does not produce all of the searched DLLs that you provided, there are two possible reasons. The first reason is that some of the DLLs you hashed in the System32 folder did not match the supplied hashes for those files stored in the NSRL file. The second reason is that you may have some corrupted DLLs on your system. Generally, the first is the case, because the list of hashes the National Software Research Library provides may not be up to date or might not even have the particular DLL.

\subsection{Check and Verify a Process Image}

The process of checking and verifying a running process's binary image follows the same approach as verifying a process's DLLs. The method of checking and verifying a process binary involves three steps: (1) identify the process, (2) identify the location of the process binary, and (3) hash the process binary and compare the hash against a known safe hash for that binary. Safe hashes could be either from NSRL library or (if you hashed all exe files on your system during a baseline collection) from a baseline system.

When you build a machine, it is a best practice to perform a baseline hash of all critical files on the system such as .exe files and DLLs so that you have a known good safe state. If an incident were to occur, you could rehash all of your critical files and compare them against your stored safe baseline set.

\section{Step 1: Identify the Process}

Identify the potential rogue process by using the process enumeration utility pslist.exe. Figure 35 displays the process that we used for this task (i.e., mshearts.exe 2840).

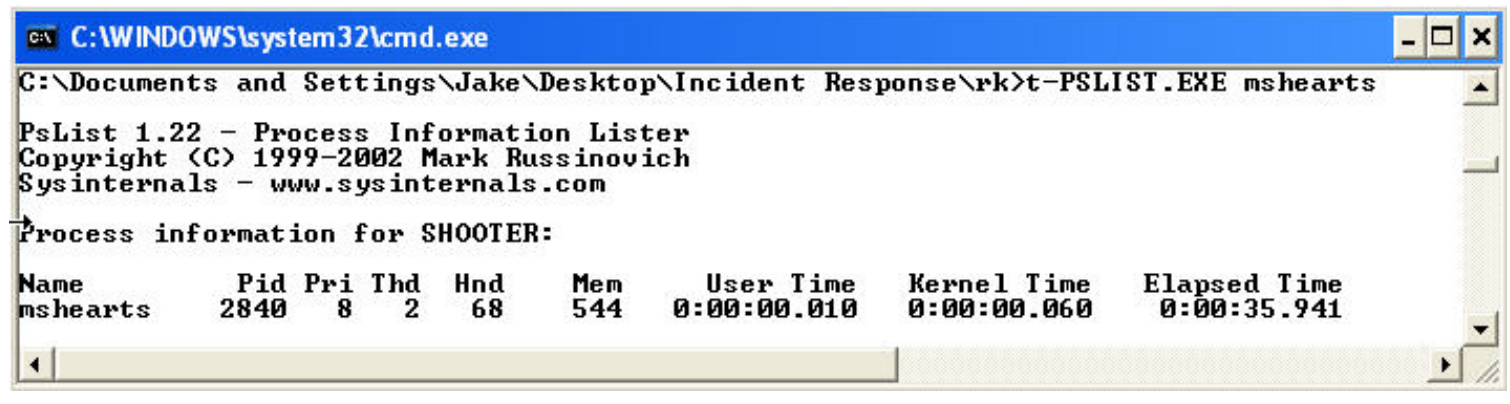

Figure 35: The mshearts.exe 2840 Process

\section{Step 2: Identify the Location of the Process Binary}

To identify the location of the process binary for the executing mshearts.exe PID 2840 process, we can use the listdlls.exe utility. Figure 36 is a screenshot of the listdlls.exe utility with the file location of the mshearts.exe binary. 


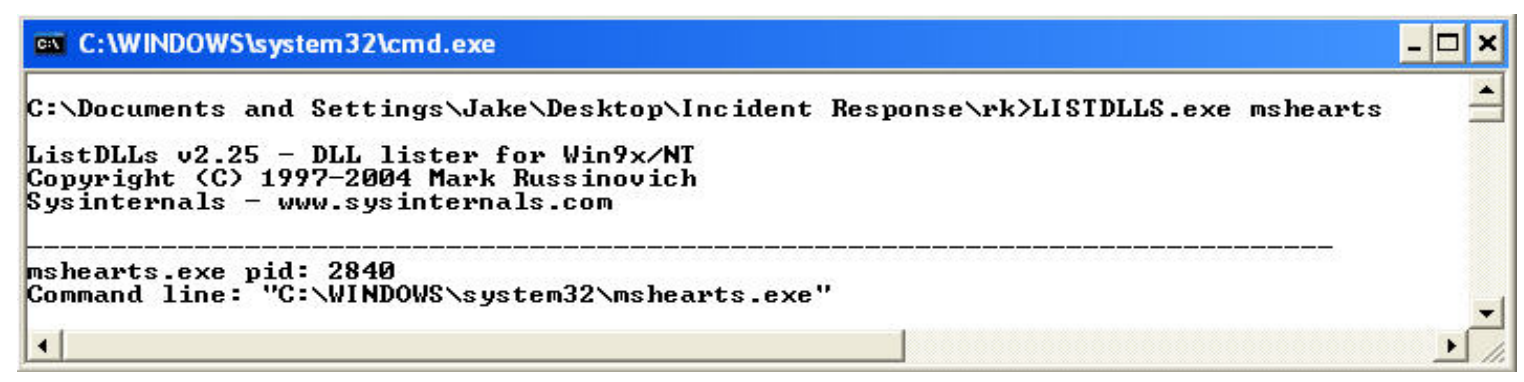

Figure 36: listdlls.exe Output for the mshearts Process

\section{Step 3: Hash the Process Binary and Compare}

Now that we have identified the process and the location of the process binary, the third and final step is to hash the mshearts.exe process binary and compare that hash against a known safe hash for the mshearts application. We use the MD5deep.exe hash utility to first hash the potential rogue mshearts.exe binary and compare the hash against the NSRL list of safe hashes for critical system files for a Windows XP system. Figure 37 is the command line syntax for using the MD5deep.exe utility to accomplish this task.

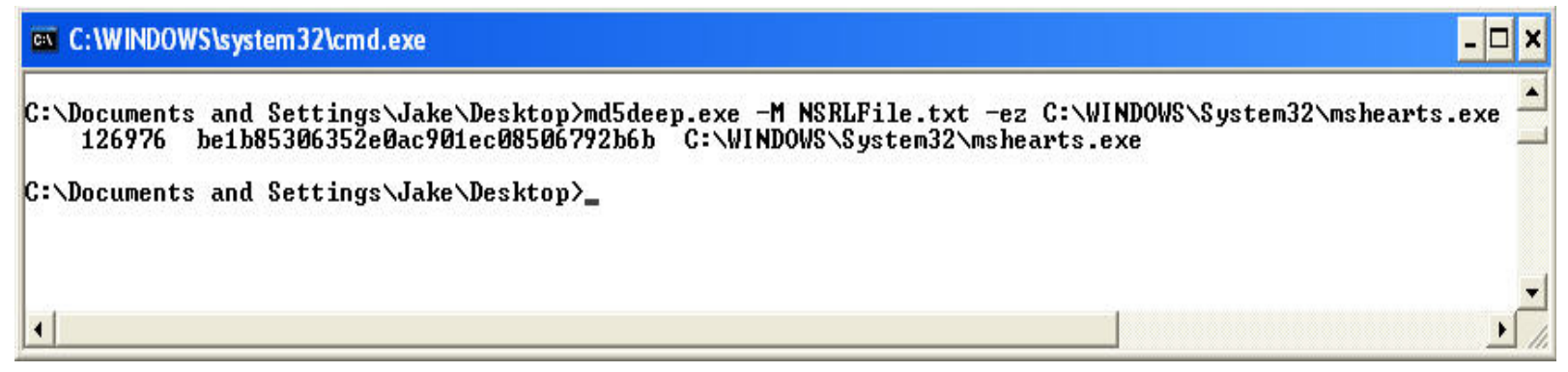

Figure 37: MD5deep.exe Command Line Arguments

As you can see, the MD5deep.exe utility computed the hash of the mshearts.exe file and, since the utility was in matching mode as defined by the $-M$ flag, the mshearts.exe hash matched against a stored hash in the NSRL text file. So now we can assume some reliability in the mshearts.exe program, since the hashes matched.

To verify this, we can perform a string search for the displayed hash (be1b85306352e0ac901ec08506792b6b) in the NSRLFile.txt file to make sure that the appropriate hash exists. 


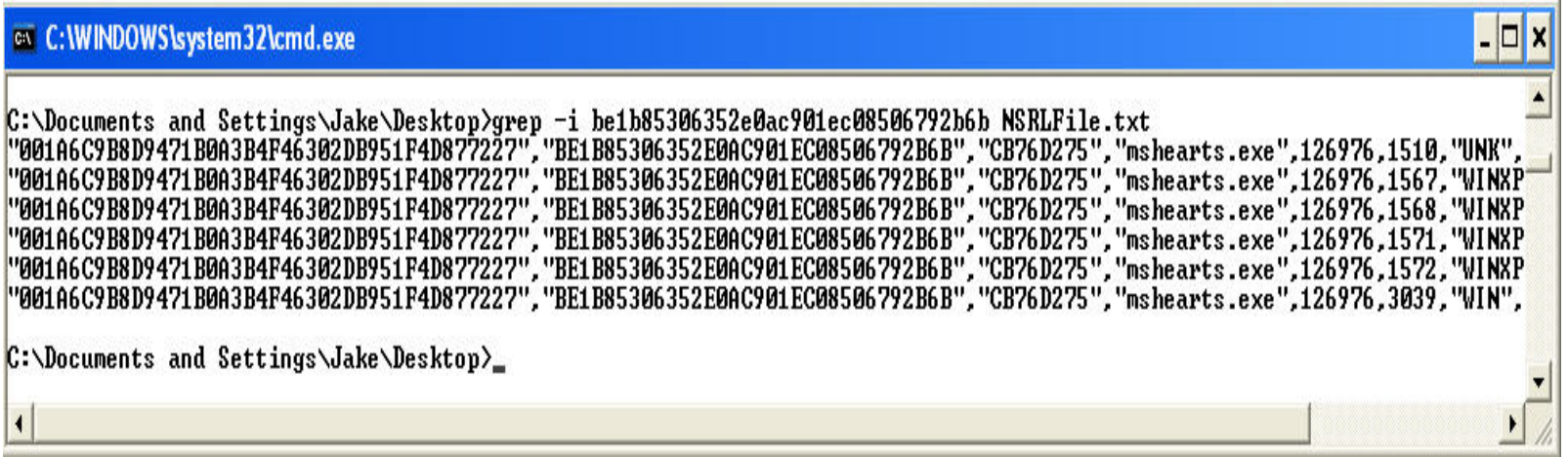

\subsection{Process String Search and Analysis}

Another method of further investigating a potential rogue process is performing a string search on the binary file to see whether you can gather additional information about the process binary and its functionality.

Performing a string search on a potential rogue binary involves a few steps. The first step is to identify the potential rogue running process and the location of its binary and then use a few command line utilities to perform a string search on the process executable. To demonstrate this, we will look at the potential rogue process called svchost1.exe.

The svchost1.exe process's binary was found to be located in the C:IWINDOWSISystem32 folder. Now that we have the location of the potential rogue process's binary, the next step is to perform a string search on the binary. To do this, we used Sysinternals' strings.exe command utility.

Working on NT and Win2K means that executables and object files will many times have embedded UNICODE strings that you cannot easily see with a standard ASCII strings or grep programs. So we decided to roll our own. Strings just scans the file you pass it for UNICODE (or ASCII) strings of a default length of 3 or more UNICODE (or ASCII) characters. Note that it works under Windows 95 as well. ${ }^{10}$

Figure 38 is a screenshot of the strings.exe utility with command line syntax used for searching for Unicode strings within the svchost1.exe binary. Note that the strings.exe command line utility will not change the access time on the file on which you choose to perform a string search. Changing the access times on files is a huge concern in computer forensics, as it relates to the admissibility of collected information.

10 http://www.sysinternals.com/utilities/strings.html 


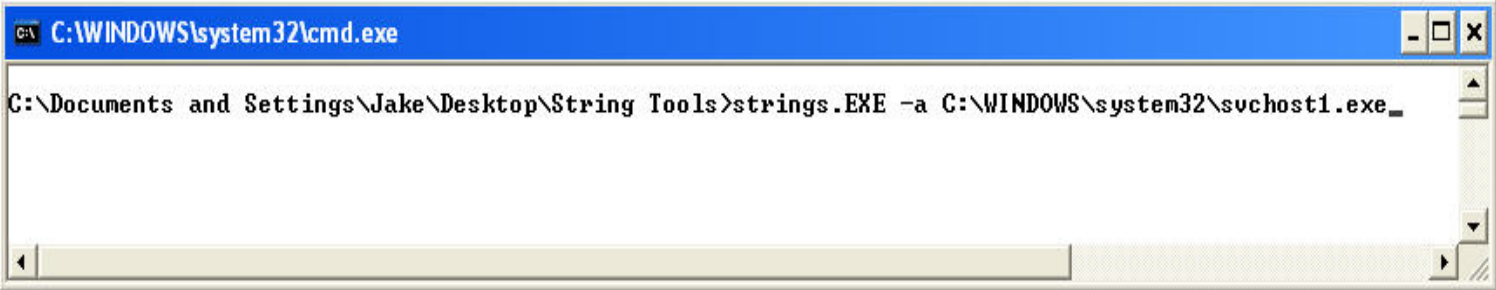

Figure 38: strings Command

Some of the identified strings that were found in the svchost1.exe binary are

- Thisisaverylongpassword

- Password355

- Longpassword

- /john.ini

Figure 39 is a screenshot of some of the more important Unicode strings that were found in the svchost1.exe process binary that led to the discovery of its actual use. As you can see, the masked svchost1.exe process is actually John the Ripper (i.e., a password cracker).

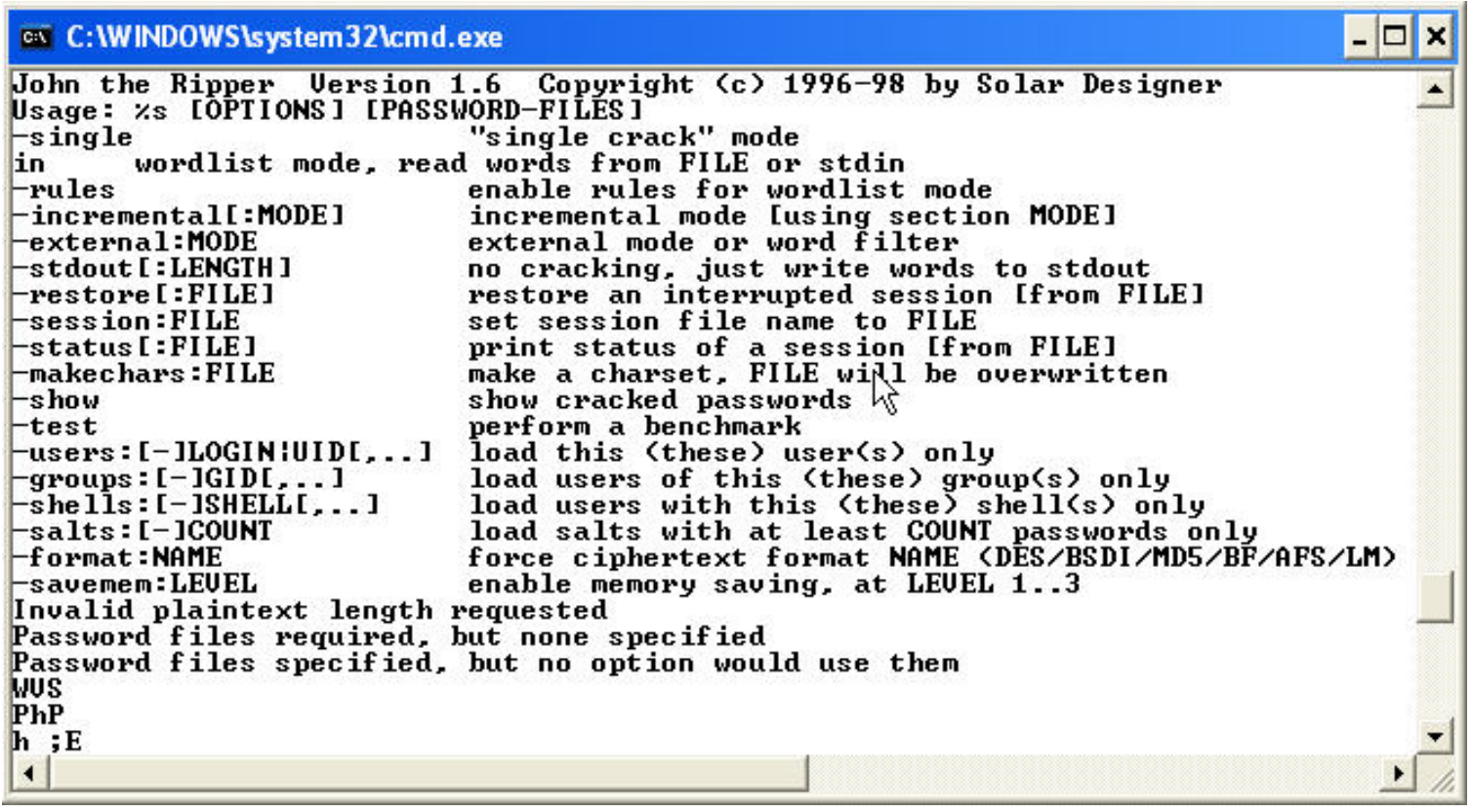

Figure 39: strings Command Output

To further verify that the svchost1.exe process is actually John the Ripper, we can hash the svchost1.exe process and see whether the hash matches the hash of a John the Ripper binary. Luckily, we can obtain the hash of John the Ripper by either downloading the hash file for the binary or downloading the binary itself from OpenWall. Figure 40 shows the hash signature for John the Ripper obtained by downloading the John the Ripper binary and hashing it with MD5deep.exe hash utility. 


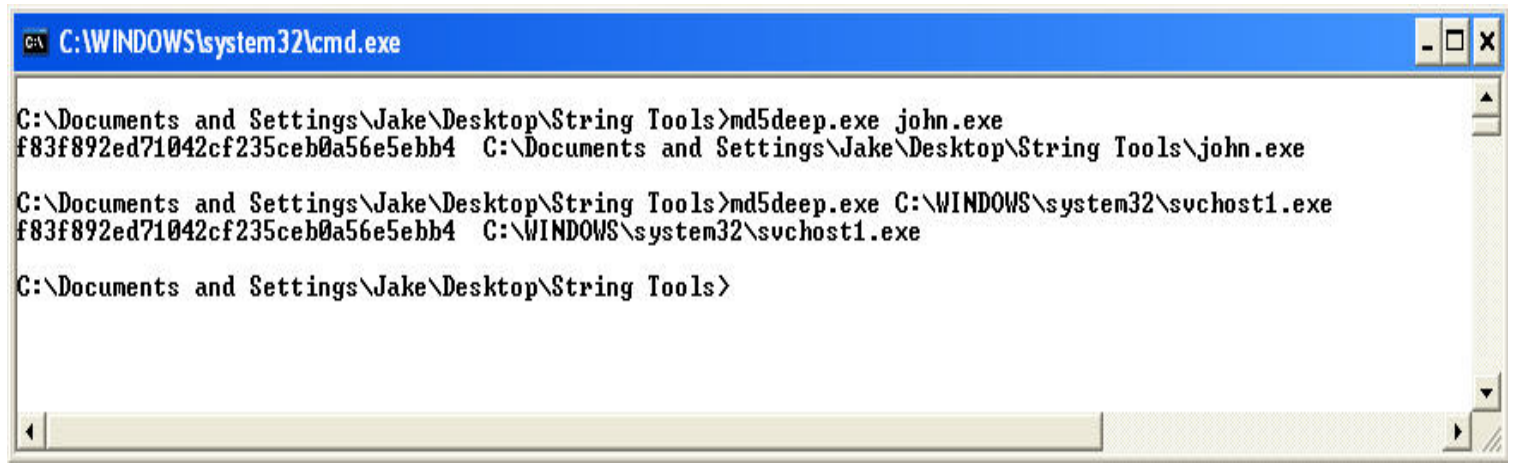

Figure 40: Hash of John the Ripper

So as you can see the hashes matched perfectly, meaning that the svchost1.exe process running on the system was actually John the Ripper. This method of performing string searches and hashing the binary can be applied to look further into any potential rogue processes that may be running. This was an easy example, however; some rogue process binaries may be stripped of Unicode text, making it harder to draw conclusions about what the process really is and its functionality. 


\section{Summary}

- Numerous process characteristics

- Difficult to distinguish between a legitimate and a non-legitimate process

- Native commands and utilities to collect the key process characteristics

- Online resources to help identify purpose or description of many Windows processes

- Baseline documentation of the system is crucial 


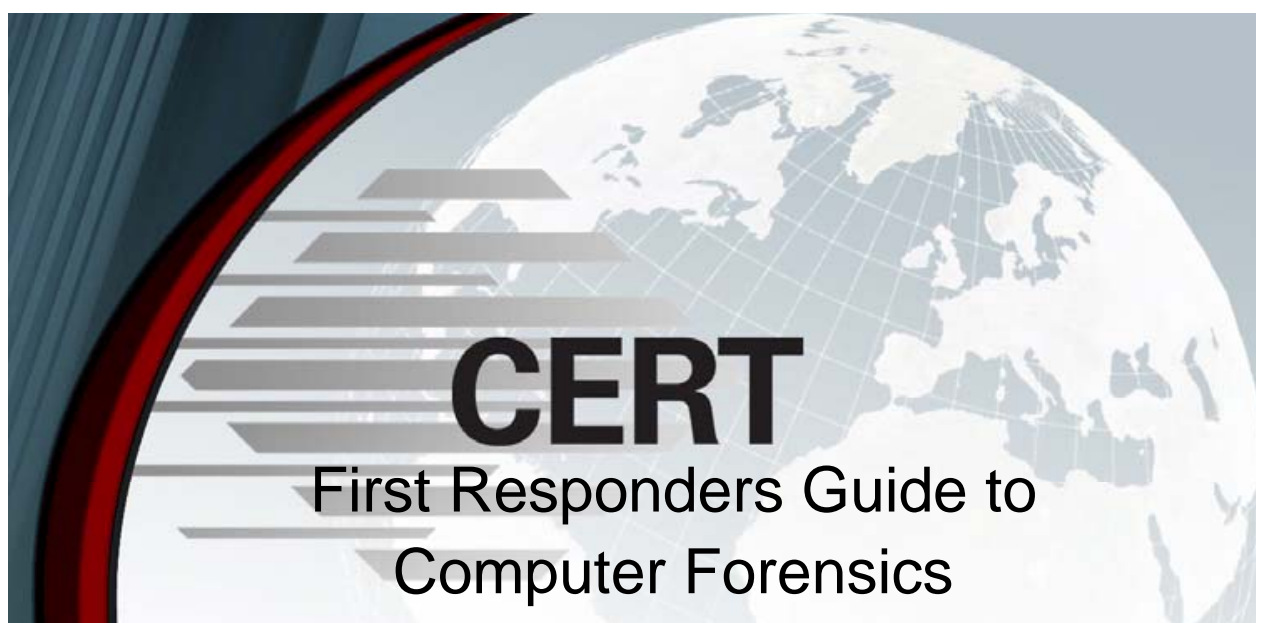

\section{Automated Process Collection}

๑) 2005 Carnegie Mellon University

Software Engineering Institute

\subsection{Automated Process Collection}

In a computer security incident situation, the last thing you want to be doing is trying to piece together a set of incident response tools to use on a possibly compromised system. Automated process collection tools like developed scripts, batch files, etc., can help automate the process of collecting forensic data from a compromised machine, as well as minimize the first responder's footprint on the system. 


\section{Objectives}

\section{First Responder Utility and Forensic Server \\ Project}

- Introduction

- Configuration

Collection of process characteristics

\subsubsection{Objectives}

In this topic, we will be looking at an automated first responder utility called FRUC and how it can be used in computer security incident situations to collect volatile data and, more specifically, some of the identified process characteristics that we pointed out in the Process Characterization topic. We will also present how to properly configure and set up both the FRUC utility and the back-end server component FSP. 


\section{What are FRUC and FSP?}

\section{FRUC (First Responder Utility)}

- Named after Harlan Carvey

- Command line interface tool

- Tool for collecting data (volatile and some nonvolatile)

FSP (Forensic Server Project)

- Tool for retrieving data (volatile and some nonvolatile)

OS Support

- Windows (2000, XP, 2003 Server)

\subsubsection{First Responder Utility (FRU)}

The automated process collection tool that we are going to present is Harlan Carvey's First Responder Utility (FRU). FRU is used by first responders to retrieve volatile data from possibly compromised systems. The current version of this utility is called FRUC, which is a command line interface tool that uses a combination of an INI file, different command line tools and utilities, and output filenames for the collected data. The FRUC utility works together with the Forensic Server Project (FSP), which is the server component of the First Responder Utility. You can use FRUC to collect and send captured data to the FSP component. 


\section{Configuration and Setup of FRUC}

\section{Components to Configure}

- Server configuration section

Server IP address \& port

1 [Configuration]

2; This section and information is required, but

3 ; can be overridden at the command line

4 server $=192.168 .2 .34$

- Command section

List of commands/utilities, args, \& output filenames

131 =fport.exe; processopenports.txt

142 handle.exe; processopenfiles.txt

$153=$ pslist.exe; processpriority.txt

- Registry section

List of registry keys and values to check

23 [Registry values]

24; Enter the Registry key, and the value you're interested in

32 [Registry Keys]

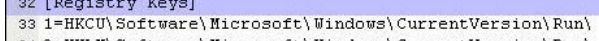

$342=\mathrm{HKLM} \backslash$ Soft ware $\backslash$ Microsof $\backslash$ Windows \CurrentVersion $\backslash$ Run

\subsubsection{First Responder Utility (FRUC) Setup}

The FRUC utility has five components that are required for the automated tool to run.

1. fruc.exe - the executable that interfaces with the fruc.ini configuration file, $p 2 x 584$.dll file, and the designated command line utilities

2. fruc.ini - the configuration file for tailoring the script to fit your collection needs. In the fruc.ini file, you will configure what commands and executables you want executed to collect volatile information.

3. P2x584.dll - the required DLL file necessary for the executable to run properly

4. list of executables - A list of executables or utilities will need to be defined so the fruc.exe utility can use them to collect volatile information. Tools like the discussed pslist.exe, psloglist.exe, and etc will need to be in the same folder as the fruc.exe utility.

5. FSP - the server component for receiving and viewing the collected volatile data

The setup for this utility to work properly requires only a few steps. The first step is to locate the fruc.ini file and tailor it to your needs. The fruc.ini file has three parts to configure.

\subsection{Step 1: Configure the Server IP Address and Port Number}

Figure 41 is a screenshot of the first part of the fruc.ini file that needs to be configured. 


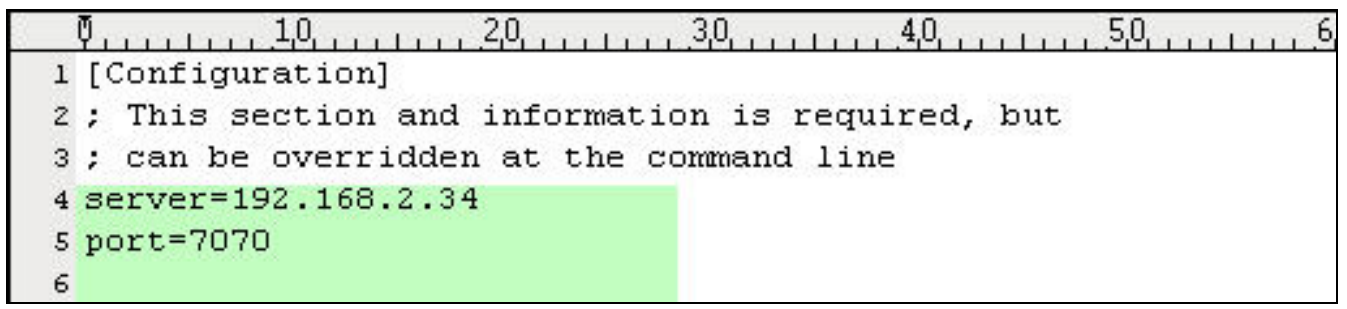

Figure 41: First Part of the fruc.ini File

The server and port settings need to be configured so that the FRUC utility knows where to send the collected volatile data (i.e., IP address of the FSP server) and on what port. Once you have this part configured, the next step is to configure the command section of the fruc.ini file.

\subsection{Step 2: Configure the Command Section}

The command section of the fruc.ini file will be the list of commands or third-party forensic utilities that will be executed in sequential order with their respected command line arguments to collect pieces of volatile data. In addition to listing the commands or utilities to be executed, you must designate a filename for the output file. Figure 42 is a screenshot of the middle part of the fruc.ini file that is to be configured.

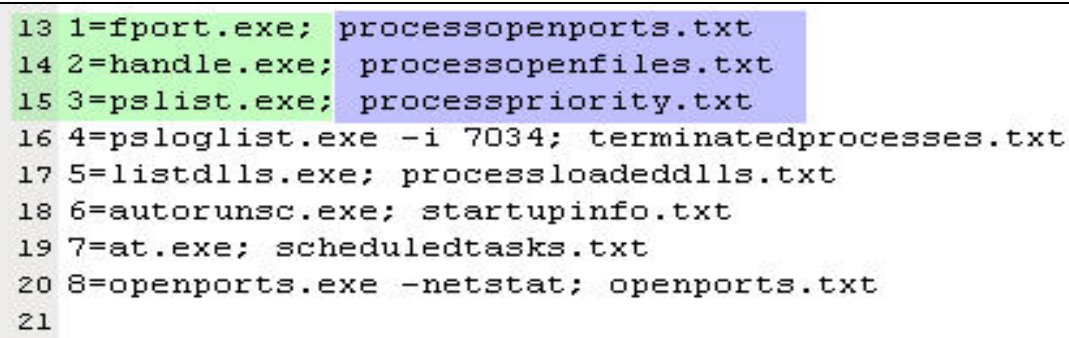

\section{Figure 42: Second Part of the fruc.ini File}

In the screenshot, the parts in green (lighter highlighting for black and white printing) are the commands or utilities to be executed in sequential order and the parts in blue (darker highlighting for black and white printing) are the names of the output files to be created to store the respective command output.

\subsection{Step 3: Configure the Registry Keys Section}

The registry keys section of the fruc.ini file will be the list of registry keys and values to collect from the compromised machine. As described, we want to check certain registry keys for auto-starting programs and services. The third part of the fruc.ini file allows us to enter a list of registry keys to check, as well as their key values. The final part of the fruc.ini file that includes a list of registry values (highlighted in green-lighter shading for black and white printing) and a list of registry keys to check (highlighted in blue-darker shading for back and white printing) is shown in Figure 43. 


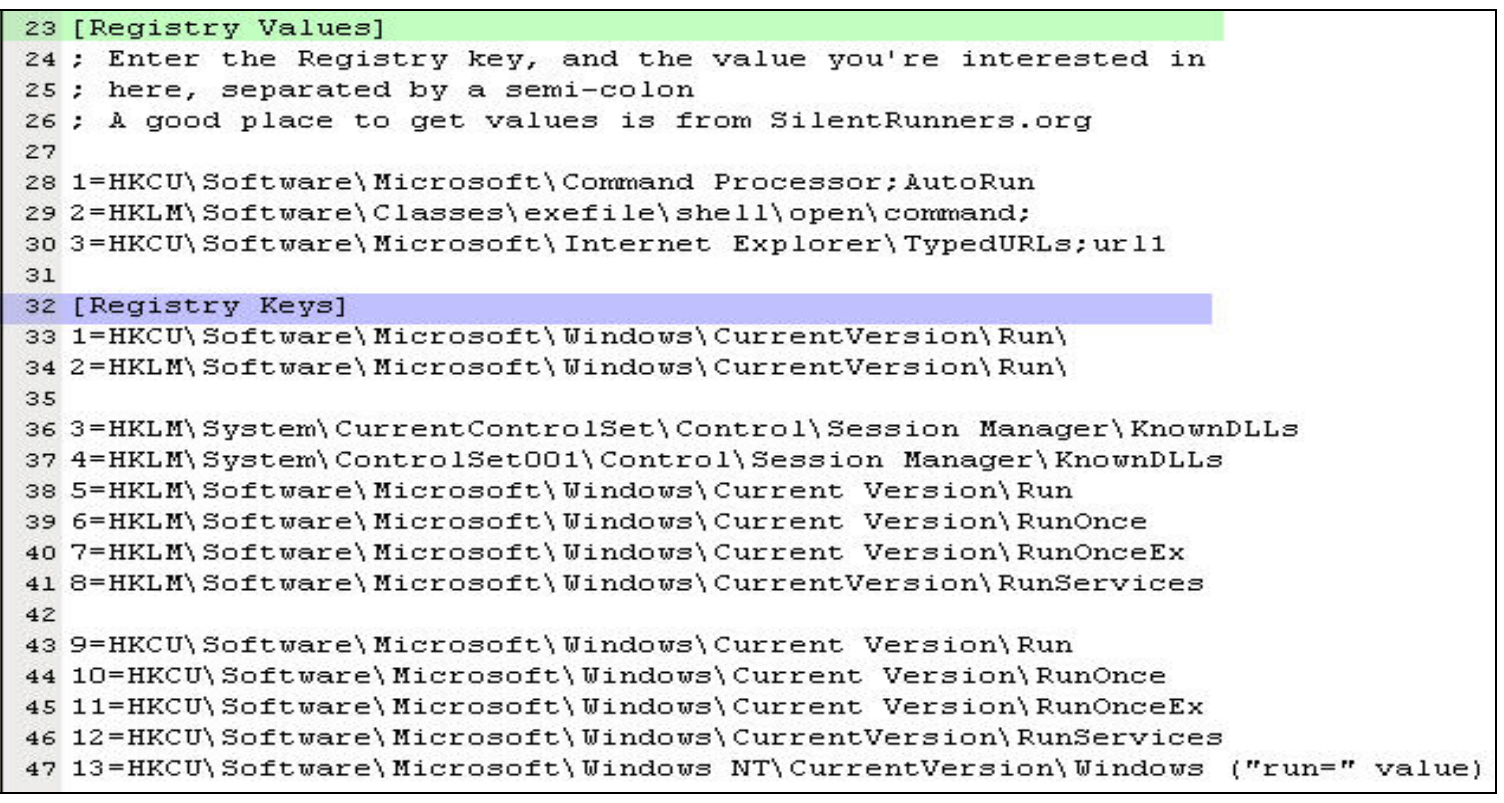

Figure 43: Final Part of fruc.ini File 


\section{Configuration and Setup of FSP}

Case directory

Name of the directory/folder where the collected data will

be sent

Case name

Name of the current incident response case

Investigator name

Name of the investigator or first responder

Port

Designated port to listen on

Logfile

Designated name for the case logfile

\subsubsection{Forensic Server Project (FSP)}

FSP server component works like a glorified netcat listener. It listens on a certain port and awaits a connection made by the First Responder Utility. Once a connection is established by FRUC, FRUC then sends the collected volatile data to the listening FSP server. Then FSP collects that data and puts it into separate files that are designated by each one of the executed commands FRUC uses.

\subsubsection{FSP Setup}

To set up FSP on the remote collection system, you will need to run the fspc.exe utility from the command line and pass it some of the configuration arguments highlighted in blue in Figure 44 (darker shading for black and white printing). 


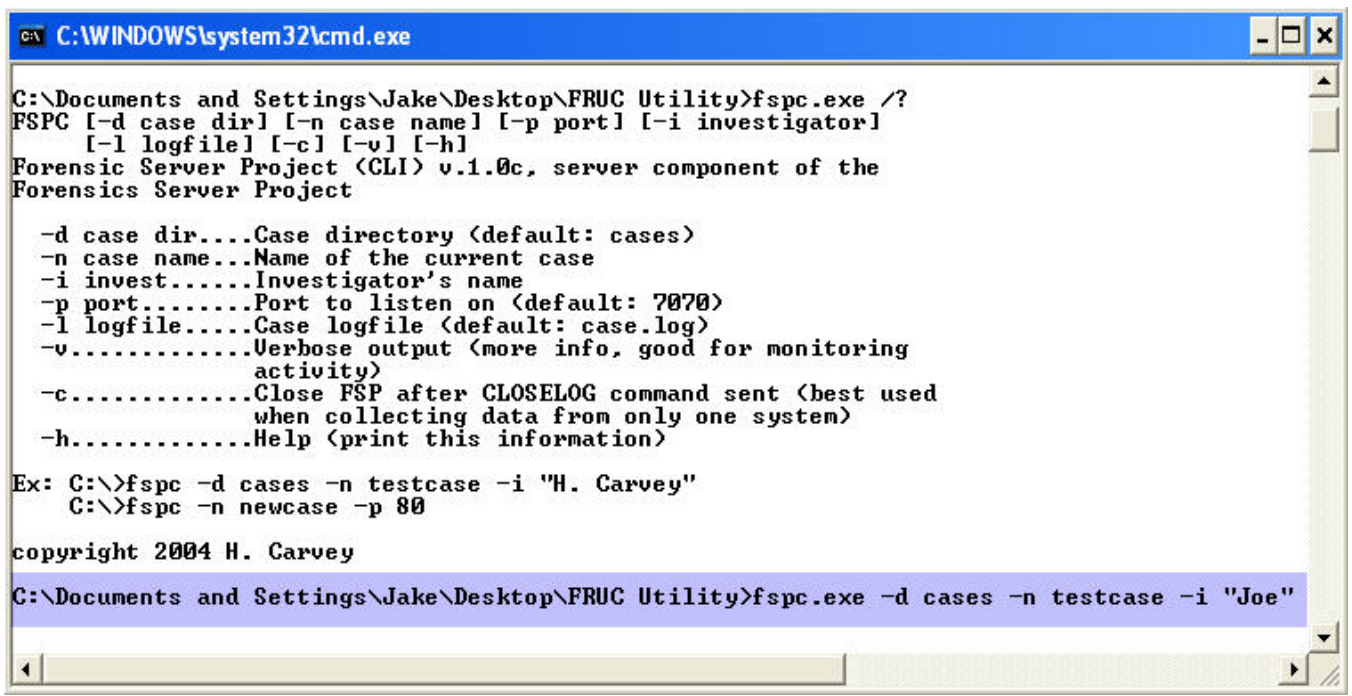

Figure 44: FSP Setup

\subsubsection{Testing FRUC}

Now that we have made all the appropriate configuration changes to the fruc.ini file, it is time to test how well this utility works. We configured the server by executing the fspc.exe utility from the command line on the remote collection system and passing it the appropriate arguments (Figure 44). We should now be able to execute the fruc.exe utility to collect volatile data and send the collected data over to the listening server. Figure 45 is a display of the fruc.exe utility being executed from the command line with the appropriate command line arguments such as the IP address to send the collected data to, on what port, which configuration file to use, and in verbose mode.

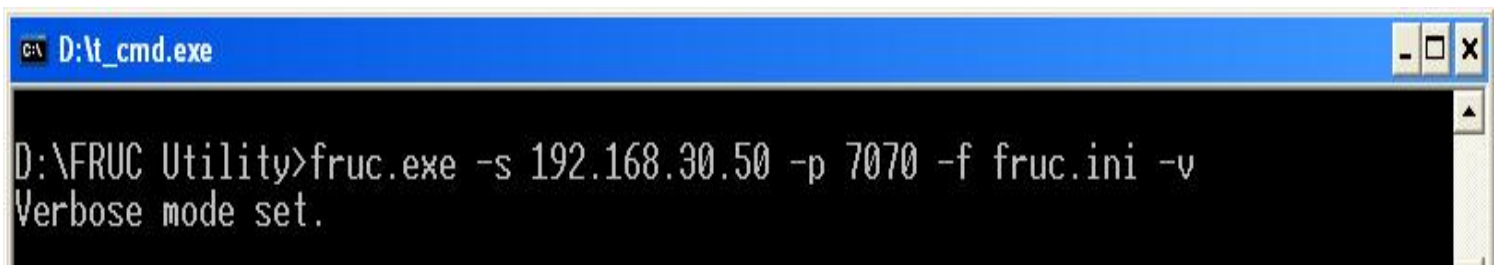

Figure 45: FRUC Utility Command 


\section{Execution of FRUC and FSP}

\section{Server component}

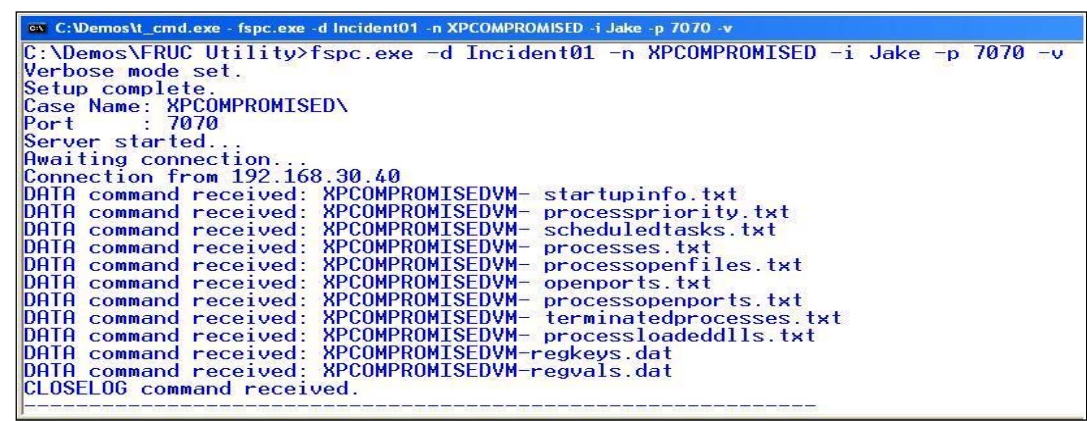

Client component

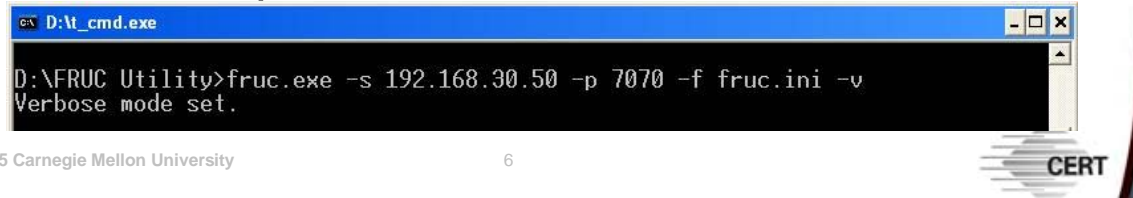

\subsubsection{Output of FRUC}

Once the server component (FSP) starts to receive the collected data sent from FRUC, it will display from the command shell that a connection has been made, what collected data has been sent over, and finally a closelog message to indicate that FRUC has stopped collecting and transmitting volatile data.

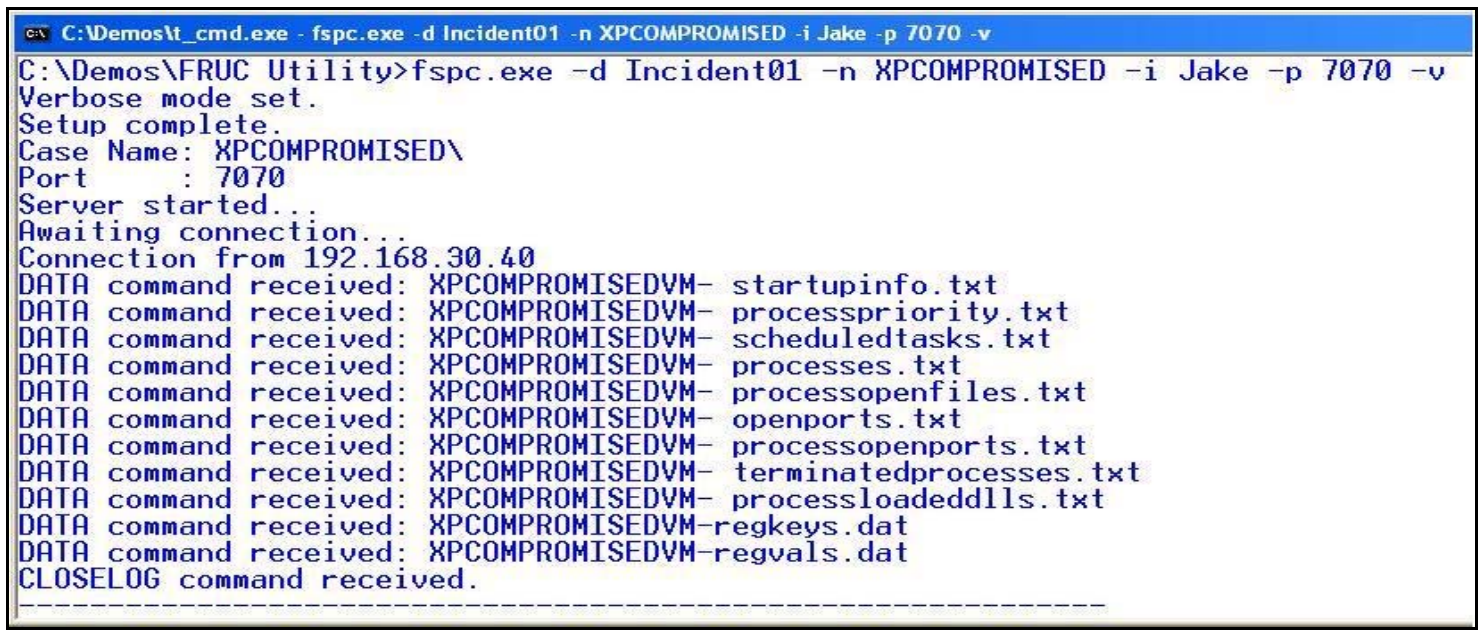

\section{Figure 46: FSP Command Output}

When the FSP server receives the collected volatile data from FRUC, it creates text files for each one of the executed commands and appends each file with the name of the system from which it is collecting the volatile data (Figure 47). So as you can see, we have a list of eight 
text files directly correlating to the eight commands that were configured to execute in the fruc.ini file. Also you can see, we have two .dat files containing a list of the registry keys and values that were defined to be collected.

\begin{tabular}{|c|c|c|c|c|c|}
\hline lakel|Desk & op'FRUC Utility'casesitestcase & & & & \\
\hline (4) & $\begin{array}{l}\text { Case } \\
\text { Text Document } \\
4 \mathrm{~KB}\end{array}$ & 骂商 & $\begin{array}{l}\text { caselog.hash } \\
\text { HASH File } \\
\text { OKB }\end{array}$ & 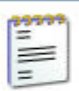 & $\begin{array}{l}\text { SHOOTER- openports } \\
\text { Text Document } \\
4 \mathrm{~KB}\end{array}$ \\
\hline$\equiv$ & $\begin{array}{l}\text { SHOOTER-processloadeddlls } \\
\text { Text Document } \\
217 \mathrm{~KB}\end{array}$ & $\equiv$ & $\begin{array}{l}\text { SHOOTER- processopenfiles } \\
\text { Text Document: } \\
104 \mathrm{~KB}\end{array}$ & $\equiv$ & $\begin{array}{l}\text { SHOOTER-processopenports } \\
\text { Text Document } \\
4 \mathrm{~KB}\end{array}$ \\
\hline$\equiv$ & $\begin{array}{l}\text { SHOOTER- processpriority } \\
\text { Text Document } \\
6 \mathrm{~KB}\end{array}$ & $\equiv$ & $\begin{array}{l}\text { SHOOTER- scheduledtasks } \\
\text { Text Document } \\
1 \mathrm{~KB}\end{array}$ & $\equiv$ & $\begin{array}{l}\text { SHOOTER- startupinfo } \\
\text { Text Document } \\
5 \mathrm{~KB}\end{array}$ \\
\hline 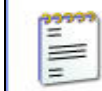 & $\begin{array}{l}\text { SHOOTER-terminatedprocesses } \\
\text { Text Document } \\
1 \mathrm{~KB}\end{array}$ & (4) & $\begin{array}{l}\text { SHOOTER-regkeys } \\
\text { DAT File } \\
3 \mathrm{~KB}\end{array}$ & (4) & $\begin{array}{l}\text { SHOOTER-regvals } \\
\text { DAT File } \\
1 \mathrm{~KB}\end{array}$ \\
\hline
\end{tabular}

\section{Figure 47: FRUC Output File}

Another great feature with this automated collection utility is what is stored in the case text file. Within the case file is an audit trail containing the time and date the server was started (as highlighted in green-lighter shading for black and white printing), the time and date each collection utility was executed (as highlighted in blue- the line containing "openports.exe”) and finally a hash of each output file (as highlighted in orange- the line containing “openports.txt”) (Figure 48).

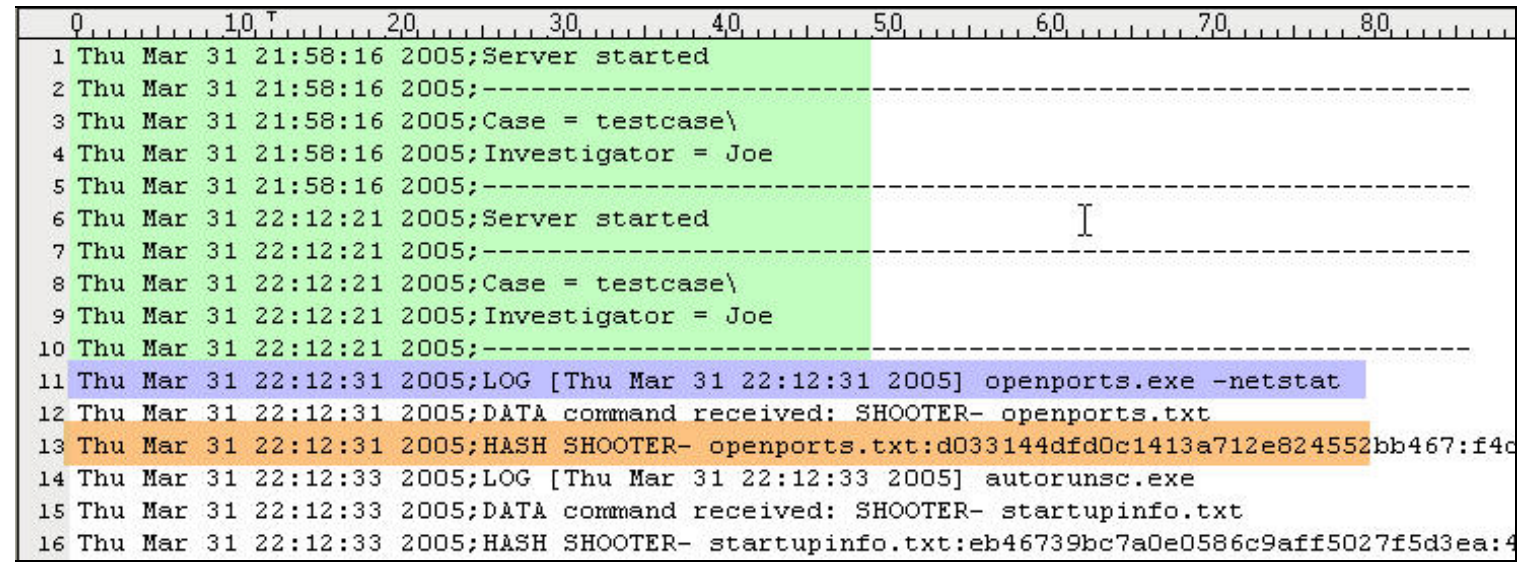

Figure 48: FRUC Audit File 


\section{Summary}

\section{FRUC}

- Versatile volatile collection tool

- Tailored to first responders' needs

FSP

- Case log acts as a forensic audit trail

- Allows for remote collection and transmission of forensic data 


\section{Module 3: Image Management}
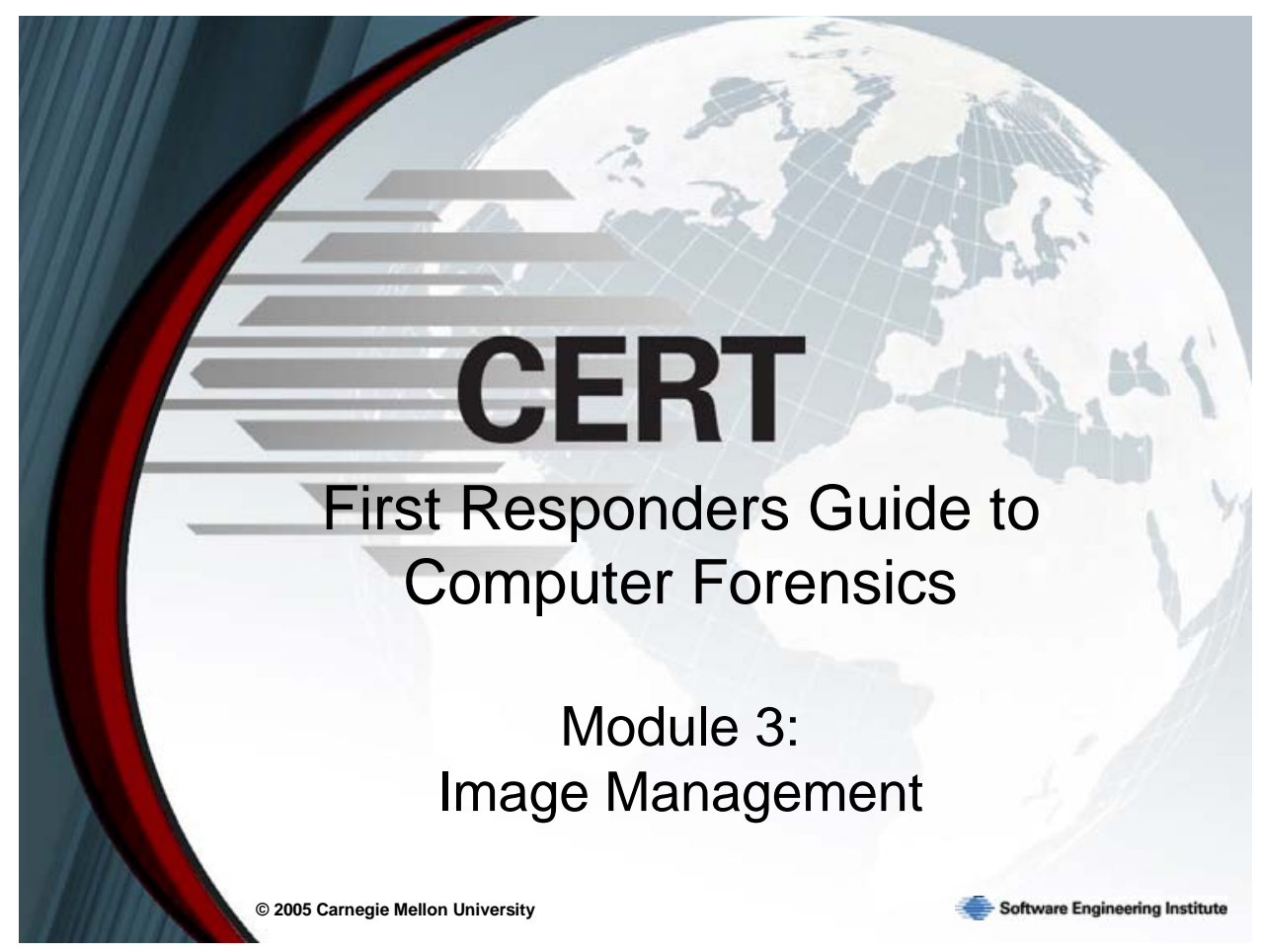

Module 3:

mage Management

In this module, we take a detailed look at capturing and restoring images and image management. Included is a discussion of the $d d$ tool, its syntax, and its variants. Reasons for splitting up blocks of data are enumerated, and techniques for breaking up an image and retrieving a specific file from within a captured image are described.

Additionally, we will walk through step-by-step instructions for two exercises. The first demonstrates how the split command can be used to break up an image. The second uses $d d$ to carve a specific file out of a captured image. 


\section{dd stands for "copy and convert"}

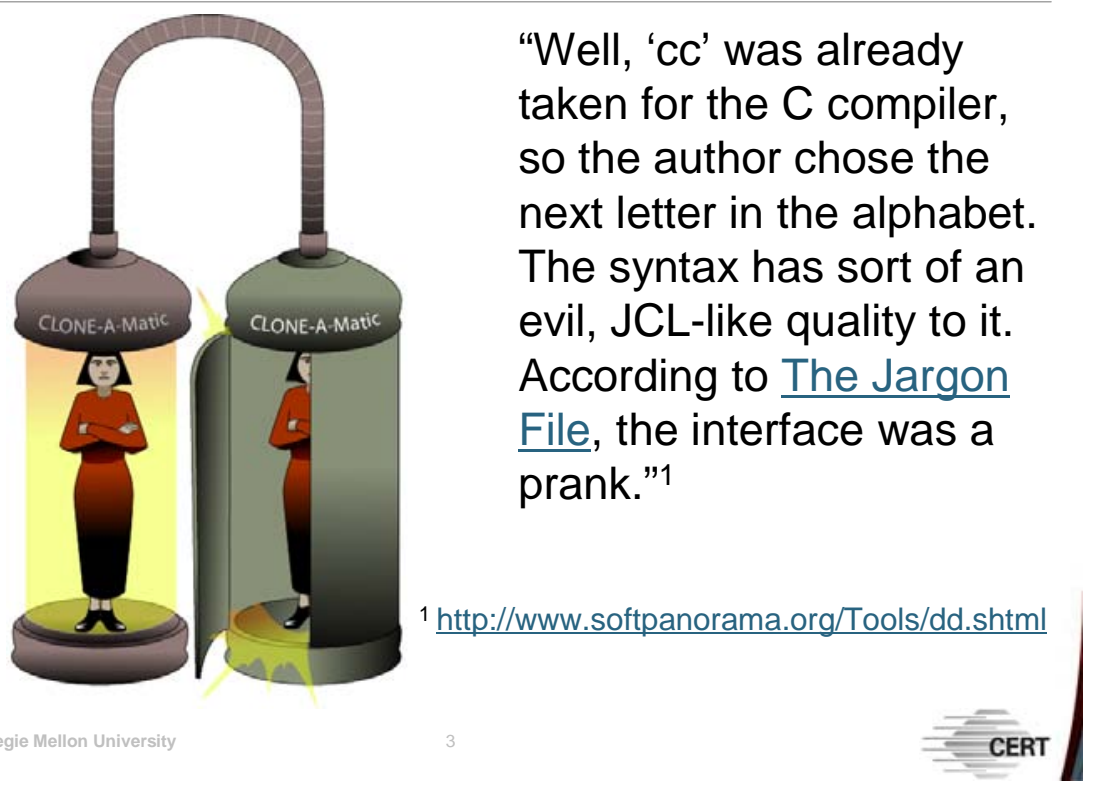

\subsection{Slice and Dice with dd}

The $d d$ tool creates bit-by-bit copies, or images, of a specified file. File, in this case, is used in the "*nix" sense of the word. A file could be anything from a floppy disk to an entire hard drive. “The name $d d$ stands for 'copy and convert.' Don't see it? Well, 'cc' was already taken for the $\mathrm{C}$ compiler, so the author chose the next letter in the alphabet. The syntax has sort of an evil, JCL-like quality to it. According to The Jargon File, the interface was a prank.”11

Initially, the syntax may look confusing, but it is pretty simple. Below is a basic example:

Table 8: dd Syntax

\begin{tabular}{|c|c|c|c|}
\hline if $=$ & file & [infile] & (i.e., read from file vs. standard input) \\
\hline of $=$ & file & [outfile] & (i.e., write to file vs. standard output) \\
\hline$i b s=$ & bytes & [input block size] & $\begin{array}{l}\text { (i.e., specify the number of bytes per read opera- } \\
\text { tion) }\end{array}$ \\
\hline obs $=$ & bytes & [output block size] & $\begin{array}{l}\text { (i.e., specify the number of bytes per write op- } \\
\text { eration) }\end{array}$ \\
\hline skip= & blocks & & $\begin{array}{l}\text { (i.e., number of blocks to skip before copying } \\
\text { starts) }\end{array}$ \\
\hline seek = & blocks & & $\begin{array}{l}\text { (i.e., number of blocks to skip before writing } \\
\text { starts) }\end{array}$ \\
\hline count $=$ & blocks & & (i.e., number of blocks to copy) \\
\hline
\end{tabular}

11 http://www.softpanorama.org/Tools/dd.shtml 
Developed for UNIX, $d d$ has since been ported to many other operating systems. There are also a few variations of the original tool. John Newbigin has written a version of $d d$ for windows. It is available at http://uranus.it.swin.edu.au/ jn/linux/rawwrite/dd.htm. Forensic Acquisition Utilities, a suite of forensic oriented applications for Windows platforms, also contains $d d$; that can be found at http://users.erols.com/gmgarner/forensics/. DCFLDD is the Department of Defense Computer Forensics Laboratory's version of $d d$, which incorporates MD5 hashing and a progress status indicator. More information on that can be found at http://www.virtualwar.com/unix/cat_computerforensics.html. 


\section{Breaking up is hard to do...NOT}

Why break up a perfectly good image?

- It is too big

- Inexpensive backup storage

- File size restrictions

Tools to use:

- split

- ma5sum

- cat

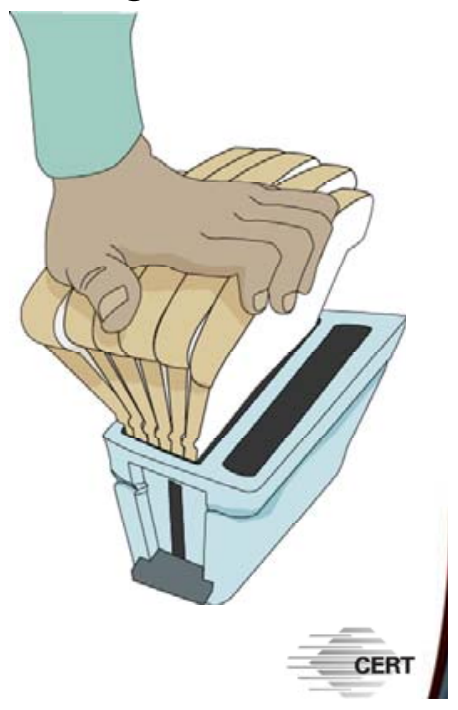

There are several reasons to break up an image. The first is the issue of having a target machine too big (think RAID server or backup tapes) to feasibly have a receptacle on hand large enough to accept the entire image. In this case, on-the-fly image splitting may be needed.

Additionally, it may be convenient to store a backup copy of the image on some non-volatile media to free up space for other activities. Splitting a 40GB image across several 5GB DVDs is one eminently practical and economically feasible option.

There are also cases when specific investigative tools have file size restrictions. In these cases a larger image must be broken up into manageable pieces for analysis. Imagine trying to toast an entire loaf of bread at one time. The results are much better if you take a slice at a time. 


\title{
Variations on a Theme
}

\section{dd, dcfldd, and dd for Windows share a similar syntax}

\author{
dd if=/*source* of $=/{ }^{*}$ destination*
}

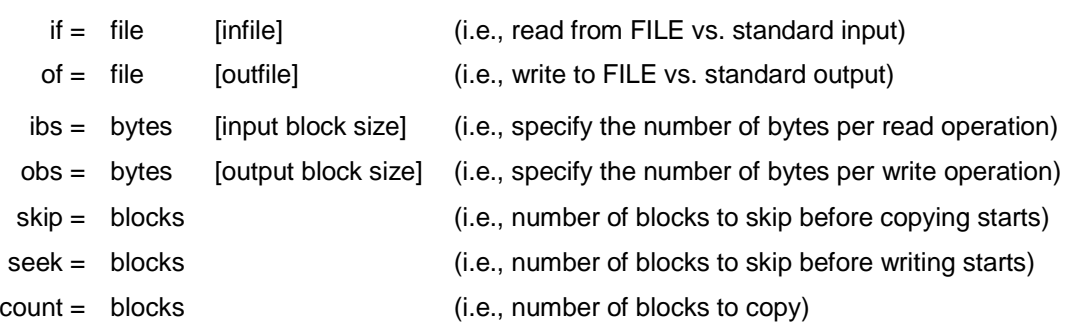

The $d d$ tool comes with most Linux distributions. Other variations that may be downloaded, such as $d c f l d d$, have enhanced features for forensics and security, including built-in MD5.

The following exercises are loosely based on the "Fun with DD" section of The Law Enforcement and Forensic Examiner Introduction to Linux: A Beginner's Guide, written by Barry Grundy. Mr. Grundy's guide is available free in PDF form online and can be found with a simple Web search.

For each $d d$ exercise, detailed directions, as well as example images, have been included so that you may see the results of each step or use them as a reference for your own hands-on experience. The first exercise will cover the basics of splitting up an image and putting it back together again. The second exercise involves carving out a specific file type from a larger image.

Splitting a $d d$ image and putting it back together again will be done in four steps:

1. Take a baseline hash of the original image.

2. Create an image in several parts (split).

3. Hash across the multiple image parts.

4. Put the image parts back together as a single image and hash.

For this example, a small Windows XP partition is used. We are going to split this partition into $2 \mathrm{MB}$ partition segments. Normally, you would have a much larger image that could be split into 2GB partitions, but for simplicity, a much smaller one is being used. Commands 
that should be entered are in shaded boxes and, in most cases, are followed by the resulting output.

First, we will use MD5 to calculate the hash value of this partition. This is used to help confirm the integrity after we have split the partition and also for when it is put it back together from the split image components to confirm that it has remained unchanged.

The filename of the Windows XP partition image being used is $x p H D . d d$. The following command will return the baseline hash value of the image:

md5sum xpHD.dd

[rooteLinuXWorkstation Demo_4]\# ls

xpHD.dd

[rooteLinuxWorkstation Demo_4]\# md5sum $\times$ pHD.dd

251c93be7a350f 148f0488b66989bcb5 xpHD.dd

[rooteL inuxWorkstation Demo_4]\#

Figure 49: Result of Using md5 to Calculate a Hash Value

We will use the split command to break the $8 \mathrm{MB}$ image into 2MB segments. Split is normally used on lines in a text file. In this case, since it is a binary file, we are using - $b$ to force the tool to deal with it as a binary file and ignore line breaks. The $2 \mathrm{~m}$ is used to specify the size of the resulting split files. Next, you specify the name of the file to be split. In this case, it's $x p H D . d d$. And finally, $x p H d$.split is the prefix of the resulting 2MB files.

split - b $2 m$ xpHD.dd xpHD.split.

Now list the files in the directory to confirm the split. You will find the original image, $x p H D . d d$, and then the four new component split images. A suffix of aa, ab, ac... is appended to the end of the file prefix for each 2MB segment.

ls $-1 h$

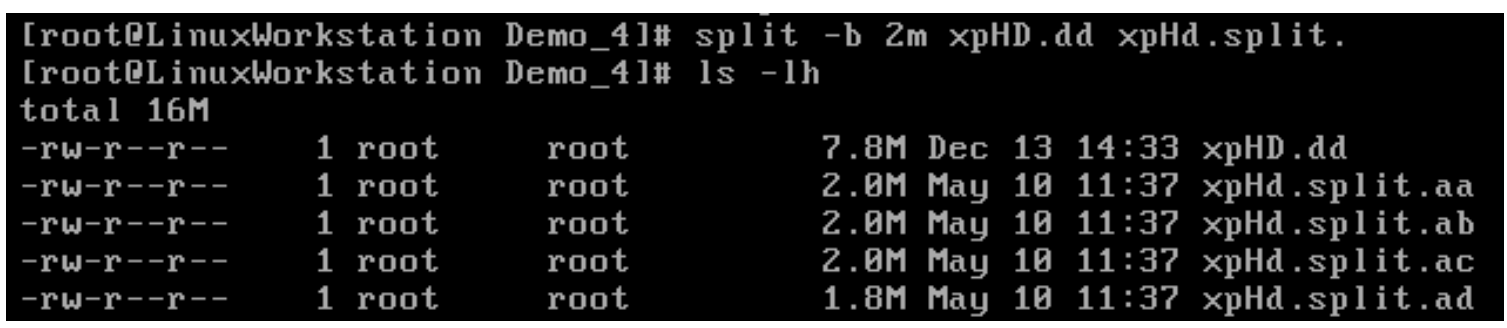

Figure 50: Confirming the Result of Splitting Images

Check the integrity of the split images using a combination of the cat and md5sum commands. The cat command will put the images back together, and then we pipe that to the md5sum tool to find the value of the split images.

cat xpHD.split.a* | md5sum 


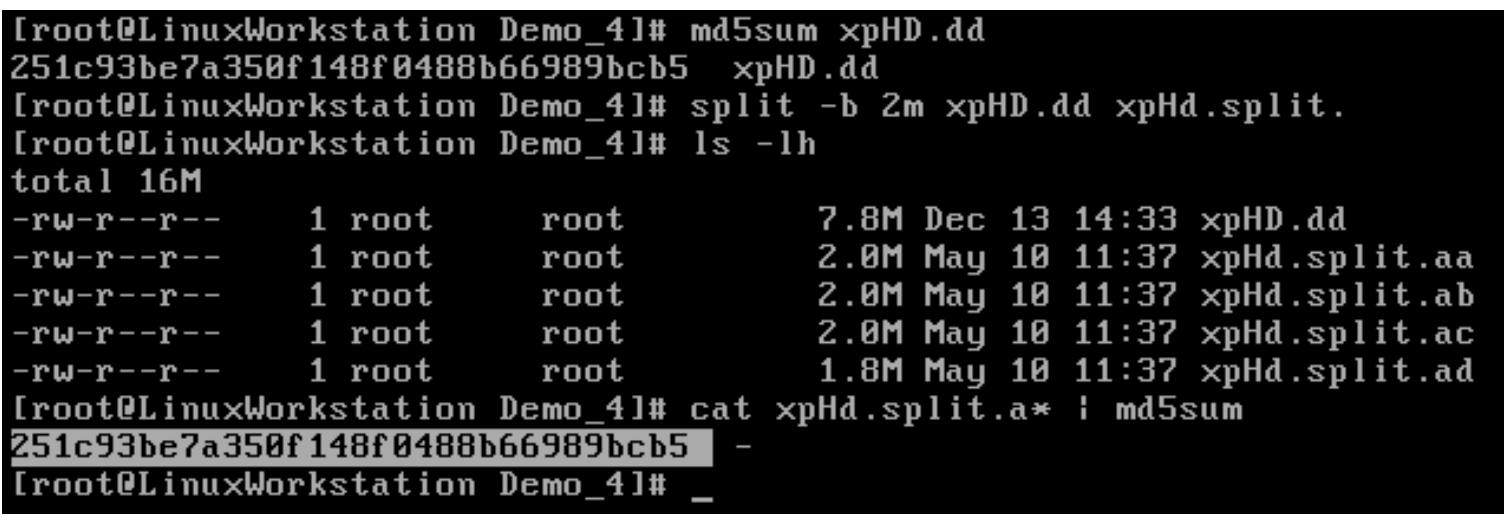

Figure 51: Result of Using cat and md5sum to Check the Integrity of Split Images

As you see, when we compare our original MD5 hash value to the new MD5 hash value, it has remained the same.

Now we will use the cat command to put the split images back together into a new file. We need to specify which files we want to put back together into a new image file.

cat xpHD.split. $a^{*}>$ xpHD.new

Do a directory listing to confirm the new image.

ls $-1 \mathrm{~h}$

Finally, check the integrity of the image that was put back together to confirm that it remains unchanged.

md5sum xpHD.new 


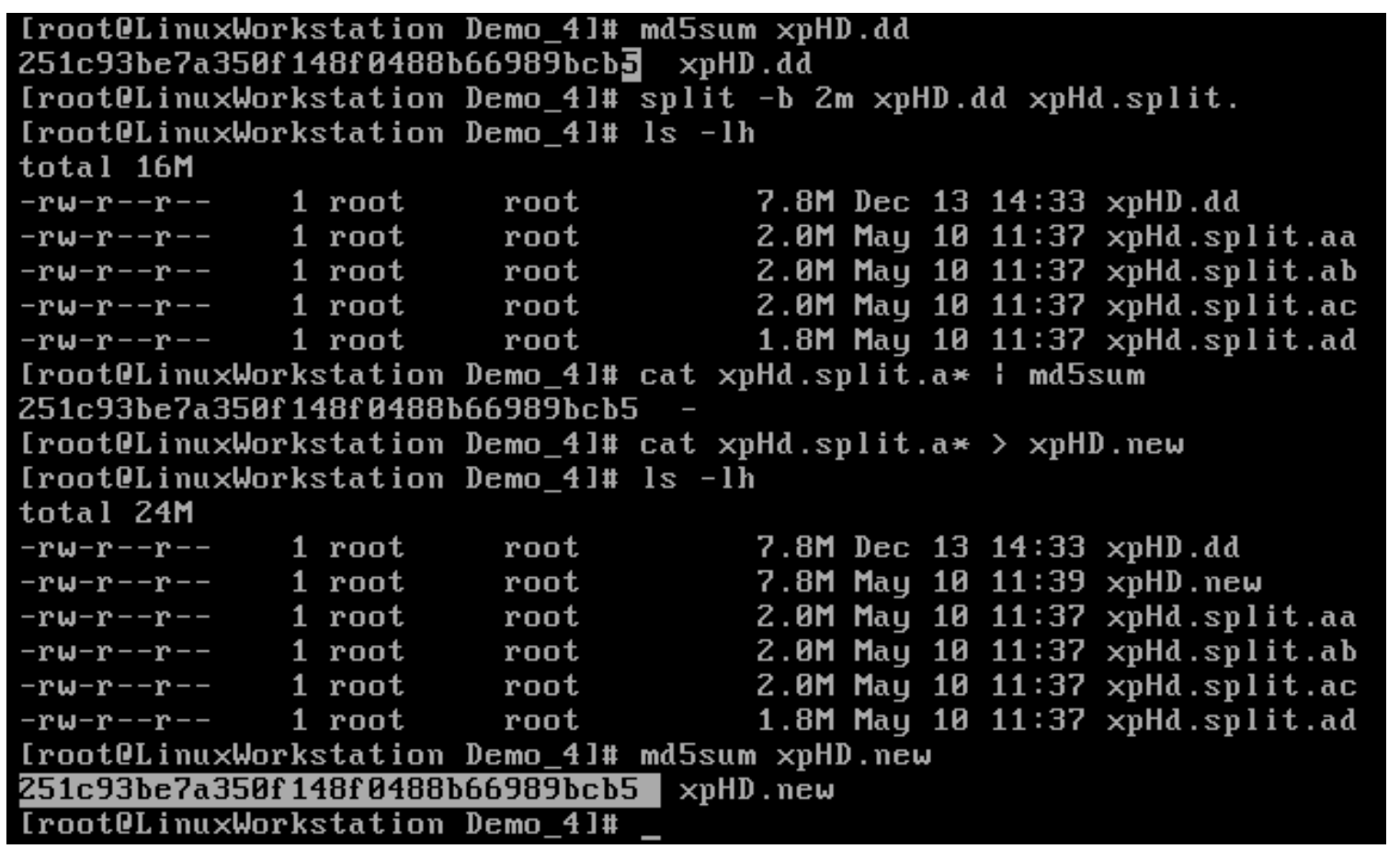

Figure 52: Result of Using md5sum to Check the Integrity of a New Image

As you can see, the value of the new hash is the same as the original file. 


\section{Data Carving}

\section{Generally done with other tools}

\section{Provides a solid understanding of what those tools are doing}

Tools to use:

- $x x d$

- grep

- $d d$

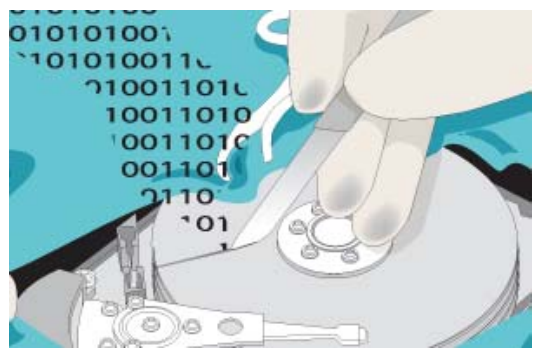

CERT

The next example will show how to carve a specific file out of a block of data.

For the purposes of this exercise, we will be using a captured image of a floppy disk to do data carving using $d d$. While this will increase your skills using $d d$, it is not the best way to go about finding a file type. There are several automated tools that work much more efficiently. Going through the process in this manner, however, gives you a good idea of how to use $d d$ in this capacity and an understanding of how the automated file searching tools actually work.

We are going to be looking in this captured image of a floppy drive for a .jpg file. In order to do that, we will start out using a hex editor to examine the floppyimage.dd image. Using grep, we are looking for the tag that delineates the beginning of a .jpg (ffd8) and finding all the places it shows up. 
[root@LinuxWorkstation flimage]\# xxd floppyImage.dd | grep ffd8 0015400: b0c3 ffd8 200d 7b69 e36a a4db 94ab cc72 .... .\{i.j....r 001ae50: c643 e8c4 c80d a304 ffd8 7a74 2db0 b89a .C............ 001e550: 2d90 efd8 ffd8 fde1 007f eef8 cdeb $0156-\ldots \ldots \ldots \ldots \ldots$. 0028930: d4c5 9413 5df4 ffd8 f076 2364 faae ba10 .............. 004ad40: bfbd 3df8 eafd f471 ffd8 fb3c f1be c2c3 ............. 0074ce0: c804 2c00 80c8 042c 0080 c8fa ffd8 $3300 \ldots, \ldots \ldots \ldots \ldots$. 00a5a40: 96af f471 ffd8 $339 f$ 2e34 2 f97 d2a6 1167 ........4/...g 00a9120: 567 c b057 ad60 fd54 b10f $80 f 7$ ffd8 af05 Vl.W. '.T....... 00ffd80: $001500 f 0$ ff3b $00000001000000000001 \ldots \ldots \ldots \ldots \ldots \ldots$ 012c260: bb7b e08f ddee 77b3 46867 c36 5b3d ffd8 .\{........6[ 6 ... 013ff20: Зеа3 7c47 ad9b bc34 b8c3 dc5f ffd8 d4b8 >.|G..4....... 01489a0: bbb7 4cdb 97bc ffd8 b07d 977d ddof b11e ..L.....\}. .... 0157400: ffd8 ffe0 $00104 a 46$ 4946_0001 $01010048 \quad \ldots \ldots$.JFIF..... H

Figure 53: Finding a .jpg Tag in a Captured Image

We are going to focus on the last line containing the .jpg tag, which reads

0157400: ffd8 ffe0 0010 4a46 $4946000101010048 \quad$.....JFIF.....H

What we have is a hexadecimal delineation of the location within the image. Translating it gives us the decimal byte offset needed to calculate the size and location of the file.

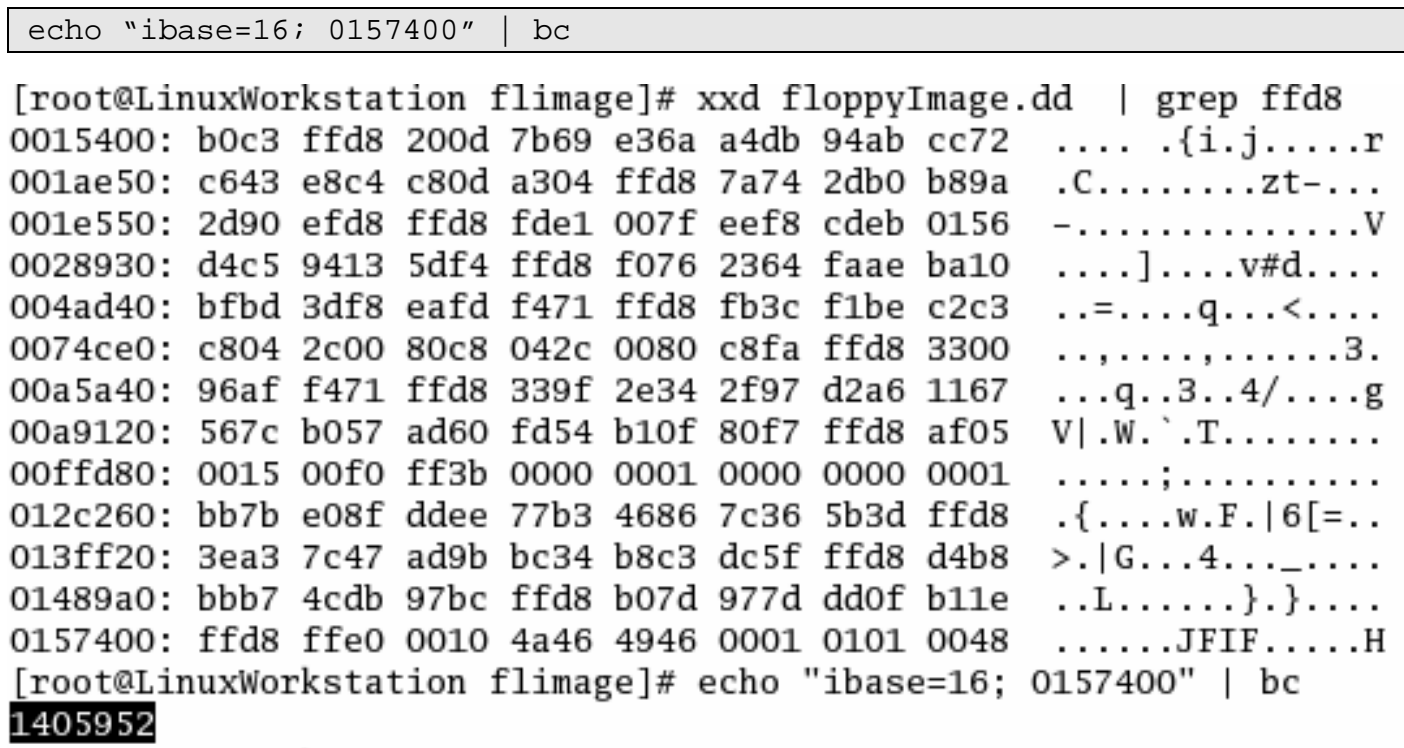

Figure 54: Decimal Form of the Beginning of the .jpg File

The result is 1405952, which is the decimal form of the beginning of the .jpg file. We will follow the same procedure with the hex editor to find the end of the .jpg. Now that we know where the .jpg file starts, we have the starting point of our search. This time we are searching for the tag that delineates the end of a .jpg file, ffd9.

xxd -s 1405952 floppyimage.dd | grep ffd9 


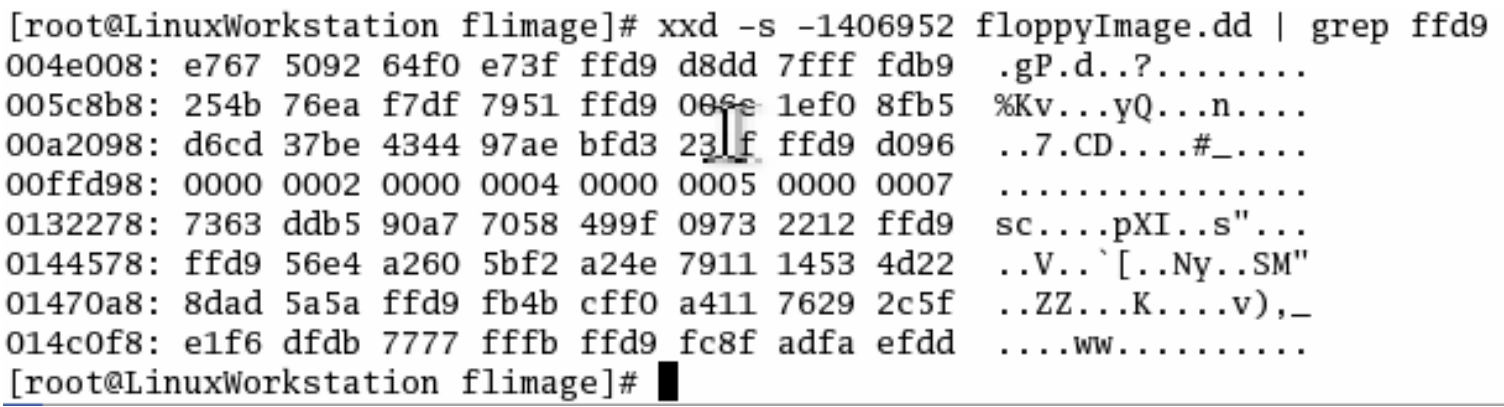

Figure 55: Searching for the End of the .jpg File

Unfortunately, with this search, we did not find anything after the value specified. This process at times can be trial and error, with several search criteria attempted before finding the desired file. We will try the search again, this time spacing the ffd9 tag.

xxd -s 1406952 floppyImage.dd | grep "ff d9"

[root@LinuxWorkstation flimage]\# xxd -s 1406952 floppyImage.dd | grep ffd9 [root@LinuxWorkstation flimage]\# xxd -s 1406952 floppyImage.dd | grep "ff d9" 0159f18: b7ff d900 $000000000000 \_000000000000 \ldots \ldots \ldots \ldots \ldots$

Figure 56: Tag Delineating the End of a .jpg File

What is returned this time is what looks like the split ffd9 tag before a bunch of blank space. We'll give that a try. Using the following command, we will find the decimal address for the ending point of what we believe to be the .jpg file.

echo "ibase=16; 0159F18" | bc

[root@LinuxWorkstation flimage]\# echo "ibase=16; 0159F18" | bc 1416984

Figure 57: Decimal Address for the End of the .jpg File

Now, to find out how large this file is, subtract the returned ending value from the starting point.

echo "1416984 - 1405952" | bc

[root@LinuxWorkstation flimage]\# echo "1416984 - 1405952" | bc 11032

Figure 58: Calculating the Size of the .jpg File

The difference, 11032, is the size of the file. We now have the starting point and the size of the file. Here is where we can use $d d$. We are going to point $d d$ at our floppy image and carve out the .jpg file (carve.jpg), skip to our starting point (1405952), take it in blocks of one (bs=1), and specify the size of the chunk to carve (11032).

dd if=floppyImage. $d d$ of=carve.jpg skip=1405952 bs=1 count=11032 
Then list the files.

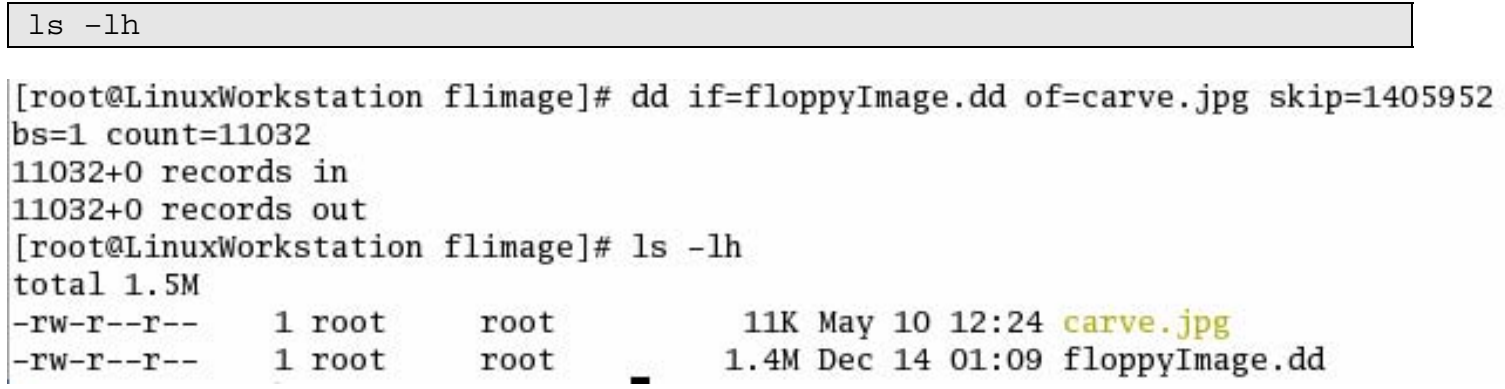

Figure 59: File Carved Out Using dd

You will find the newly carved carve.jpg file. Use a tool such as xview to view the image.

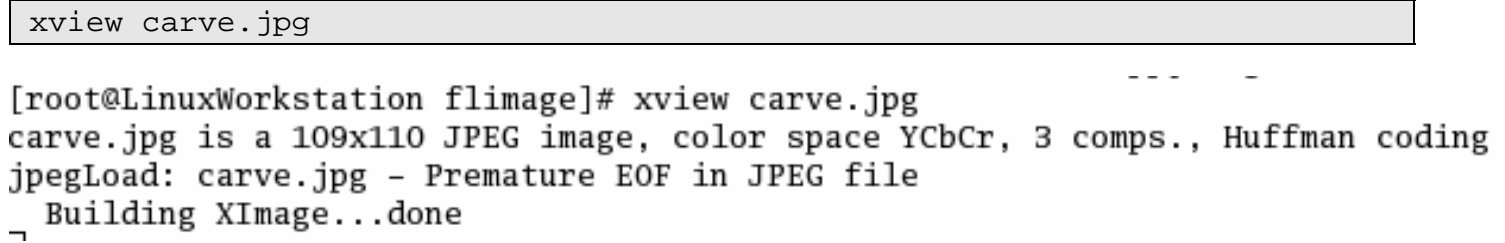

Figure 60: Viewing Carved .jpg File 


\section{Summary}

\section{Images are split for a variety of reasons}

- Available resources

- Backup storage

- Tool limitations

\section{dd can be more than just a collection tool...} but that doesn't mean it should be.

There are many reasons to split an image into smaller pieces. Adopting the strategy that is right for any specific situation depends on understanding the rationale behind these actions. As always, efficient and effective response to an actual security incident is largely a function of the quality of preparation carried out beforehand.

In review, this exercise showed us how to identify the beginning and the end of a file and how to use $d d$ to carve out that file from within a captured image. Again, this process would not normally be done. It was used as an explanation of how $d d$ and other tools work. Automated tools such as Autopsy and The Sleuth Kit will automatically identify file types and where files are located and will allow you to access the files separately from within the captured image. 


\title{
4 Module 4: Capturing a Running Process
}

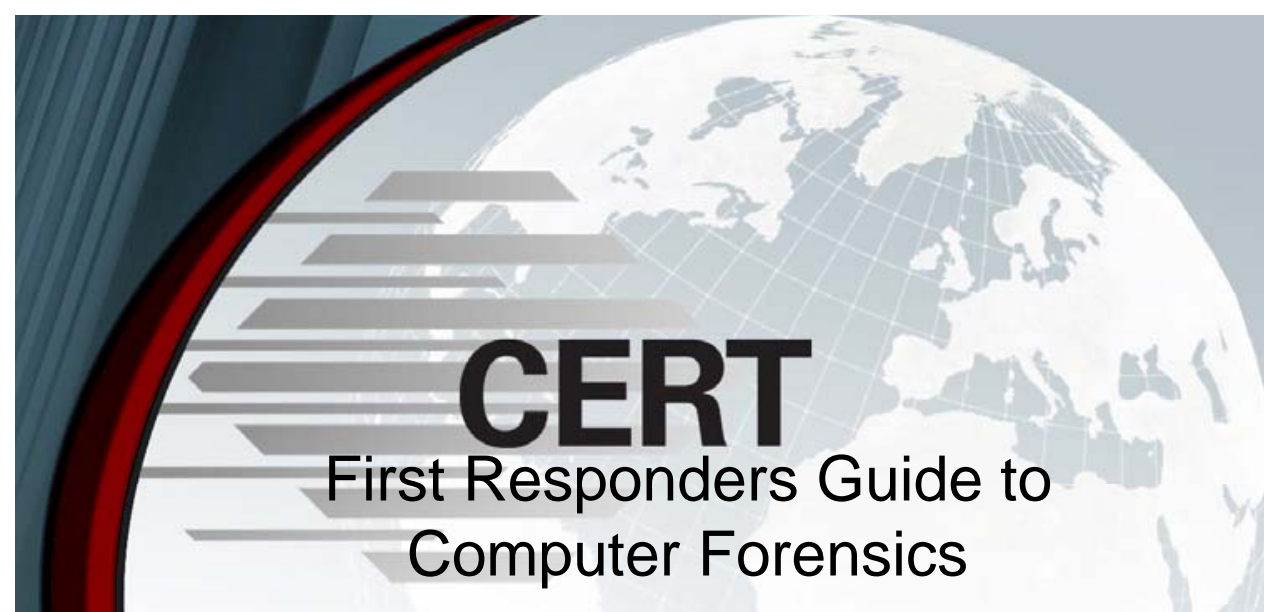

\author{
Module 4: \\ Capturing a Running Process
}

○) 2005 Carnegie Mellon University

들 Software Engineering Institute

This module sets forth one technique for capturing a suspicious process from a live machine (there are other ways to perform such a capture). The important conceptual take-away from this module is to approach problems of this nature with a forensic mindset. Specifically, take pains to leave as small a footprint on the suspect machine as possible. This requires both technical and procedural preparation. 


\section{Objectives}

Discuss the benefits and drawbacks of

capturing a process from a live machine

Learn to capture a suspicious process on a live Windows machine

Learn to capture a suspicious process on a live Linux machine

The primary purpose of this module is to demonstrate how to capture a suspicious process from a live machine. ${ }^{12}$ Both Windows and Linux platforms are addressed.

As you collect data (i.e., potential evidence) from a live computer, consider the data's order of volatility: that is, collect data that has the highest chance of being changed, modified, or lost first. The order of volatility for Windows and Linux computers is the same. ${ }^{13}$

12 The ability to perform such a capture depends on a foundation of knowledge not contained in this section. For example, no instruction regarding the creation of a response disk consisting of safe collection tools is offered in this section.

13 Brezinski, D. Guidelines for Evidence Collection and Archiving (Network Working Group RFC 3227). http://www.ietf.org/rfc/rfc3227.txt (2002). 


\section{Capture a Running Process}

Hedons (Pros)

- Keeps system live

- Facilitates troubleshooting

- Can collect individually targeted processes

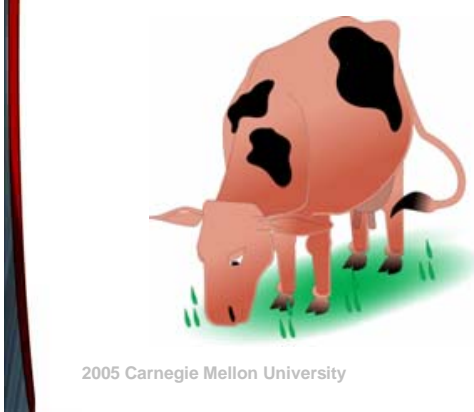

Dolors (Cons)

- Leaves a footprint

- May corrupt volatile data

- Can be difficult to identify malicious processes

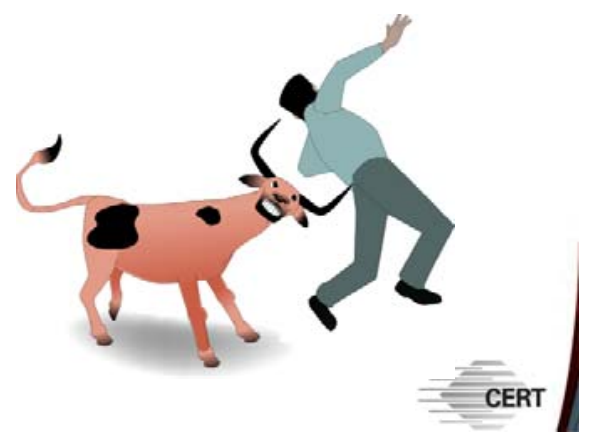

\subsubsection{Hedons and Dolors}

Hedon is a term that utilitarians use to designate a unit of pleasure. Its opposite is a dolor, which is a unit of pain or displeasure. There are some significant hedons associated with capturing a process from a running system. An important factor is that the system remains running. There are times when it is just not feasible to shut down a system. The ability to pull off a suspicious process for further analysis facilitates troubleshooting without sacrificing time. Additionally, it is possible to target specific processes.

It is important to remember that every silver lining has its cloud. Unlike an examination of a dead host, any action taken on a live machine leaves a footprint. The actions taken to extract the suspicious process may end up corrupting evidence. While the techniques discussed below allow for targeted extraction, it may be difficult to know exactly what to capture. There are many processes running on a machine and their names do not always provide a clear idea of what they do. Knowing what processes the machine normally runs greatly increases the chances of identifying the ones that should not be there. 


\section{Windows System}

\section{Tools}

- Netcat, PsList, ListDLLs, dd, md5sum

Prep work

- Create response CD

- Verify someone else's response disk

\section{Steps}

- Identify suspicous processes

- Use ListDLLs to determine path of executable

- Copy out process using $d d$ and $n c$
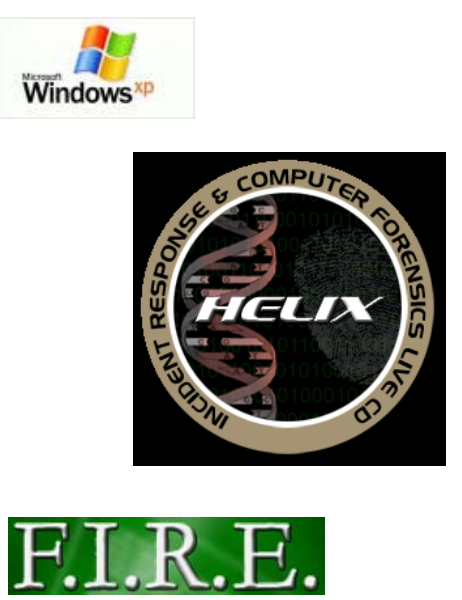

\subsubsection{Capturing a Process on a Windows System}

Table 9 shows a list of tools that can be used to capture a suspicious process on a live Windows system, as well as step by step instructions for performing the capture.

Forensic collection best practices dictate that programs on the suspect machine are not to be trusted. The tools for the collection should be put on a response disk (most likely a CD).

Table 9: $\quad$ Tools for Capturing Running Processes

\begin{tabular}{|l|l|}
\hline Tool & Description \\
\hline PsList & $\begin{array}{l}\text { PsList is utility that shows you a combination of the information obtainable individually } \\
\text { with pmon and pstat. You can view process CPU and memory information or thread sta- } \\
\text { tistics. What makes PsList so powerful is that you can view process and thread statistics } \\
\text { on a remote computer. }{ }^{14} \text { Go to http://www.sysinternals.com/ntw2k/freeware/pslist.shtml } \\
\text { to download the tool and obtain installation instructions. }\end{array}$ \\
\hline
\end{tabular}

14 Russinovich, Mark. PsList. http://www.sysinternals.com/ntw2k/freeware/pslist.shtml (2004). 


\begin{tabular}{|l|l|}
\hline Tool & Description \\
\hline ListDLLs & $\begin{array}{l}\text { ListDLLs shows you the full path names of loaded DLL modules. In addition, ListDLLs } \\
\text { will flag loaded DLLs that have different version numbers than their corresponding on- } \\
\text { disk files (which occurs when the file is updated after a program loads the DLL) and can } \\
\text { tell you which DLLs were relocated because they are not loaded at their base address. } \\
\text { Go to http://www.sysinternals.com/ntw2k/freeware/listdlls.shtml to download the tool } \\
\text { and obtain installation instructions. }\end{array}$ \\
\hline$d d$ & $\begin{array}{l}\text { dd (discussed in Section 3.1) is an imaging tool. It makes a bit-by-bit copy (forensic im- } \\
\text { age) of the target data. The target could be an entire hard drive, a specified partition, or } \\
\text { even the physical memory. For the purposes of this module, dd will be used to make an } \\
\text { image of an executable. Go to http://uranus.it.swin.edu.au/ jn/linux/rawwrite/dd.htm to } \\
\text { download the tool and obtain installation instructions. }\end{array}$ \\
\hline NetCat & $\begin{array}{l}\text { Netcat is a featured networking utility that reads and writes data across network connec- } \\
\text { tions using the TCP/IP protocol. It is designed to be a reliable back-end tool that can be } \\
\text { used directly or easily driven by other programs and scripts. At the same time, it is a fea- } \\
\text { ture-rich network debugging and exploration tool, since it can create almost any kind of } \\
\text { connection you would need and has several interesting built-in capabilities. }\end{array}$ \\
\hline
\end{tabular}

Before capturing the suspicious process, prepare your response CD of safe tools for the platform from which the capture will be performed. An alternative to creating your own response CD is to use one already created. HELIX is an example of such a disk. ${ }^{17}$ In addition to being a bootable Linux environment for incident response, the disk also contains many useful tools for examining a live Windows host. FIRE is a similar resource; for more information visit http://biatchux.dmzs.com/?section=main or refer to the First Responders Guide to Computer Forensics [Nolan 05].

For the following example, two separate machines are needed. The first machine, a Windows XP box, will act as the compromised system and will be referred to as the "target machine." This target machine has an IP address of 192.168.30.20. The second machine, a host running the WhiteBox flavor of Linux, will function and be referred to as the "collection machine." It has an IP address of 192.168.30.50. Information will be captured from the target machine and sent to the collection machine for analysis. This example can be reproduced on any two machines connected over a network if the user has created a response CD compatible with the host operating systems and substitutes the IP addresses used in this example with the IP addresses of the machines being used.

The response CD used in this example contains trusted tools used in the capturing process. The tools have been renamed with a " $\mathrm{t}$ _" for clarity. For example, the executable file to call

15 Russinovich, Mark. ListDDLs. http://www.sysinternals.com/ ntw2k/freeware/listdlls.shtml (2000).

16 Giacobbi, Giovanni. NetCat. http://netcat.sourceforge.net/ (2004)

17 http://www.e-fense.com/helix/ 
up a command window has been renamed from $\mathrm{cmd}$.exe to $t$ _cmd.exe. This ensures that the tool or application being used is from the response $\mathrm{CD}$ and not the local machine.

Now we begin the process.

1. Insert your response disk into the target machine.

2. Click the "Start" button.

3. Click "Run."

4. Enter the path to the tool to be used. In this example, the $\mathrm{CD}$ drive is the $\mathrm{D}: 1$ drive and we are using t_cmd.exe as a trusted command shell in the WTools folder.

A command shell will open. Please note the “D:IWTools $\backslash$ _cmd.exe" at the top of the command

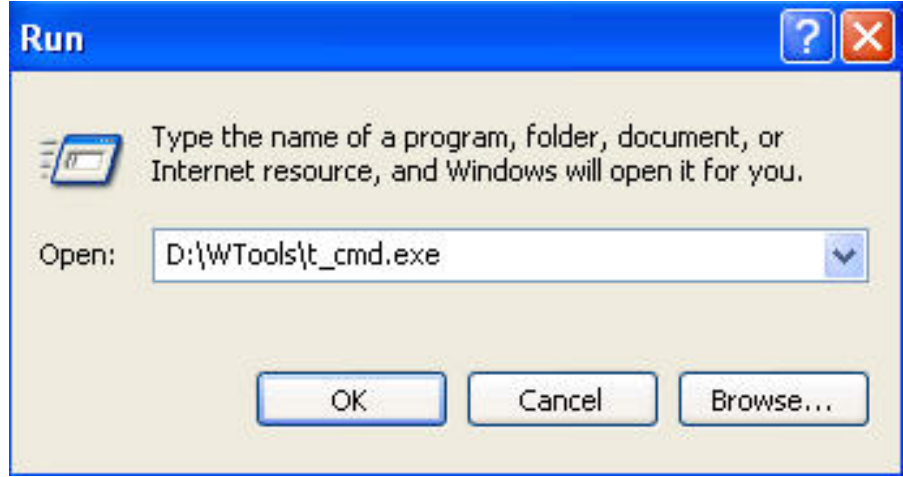

Figure 61: Running a Trusted Command shell. This indicates that the command shell is, in fact, spawned from the trusted CD.

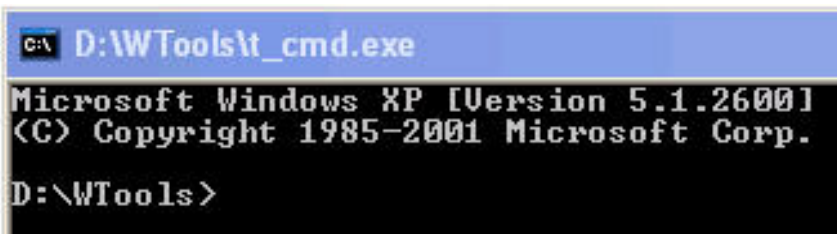

Figure 62: Command Shell Spawned from a Trusted CD

5. On the collection system, set up a netcat listener to receive the results from the pslist you will run on the target machine by typing the command below.

nc $-1-$ p $3333>$ pslistText.txt

The netcat application is called with nc, - 1 tells netcat to listen, - $\mathrm{p}$ denotes what port to listen on. Any port above the reserved ports 1-1024 can be selected for netcat to listen on. We chose 3333, as it's easier to keep and audit separate from other common ports. Finally, anything received on port 3333 will be written to the file pslistText.txt.

[rooteL inuXWorkstation data]\#
[rooteLinuxWorkstation data]\# nc - - p $3333>$ pslistText.txt_

Figure 63: netcat Command to Listen on Port 3333

6. On the target machine, run the trusted pslist command and pipe the results to the collection machine via netcat using the command below. 
t_pslist.exe | t_nc.exe 192.168.30.50 3333

We are using the trusted pslist and netcat tools from the resource CD, as denoted by the "t_." To send the results to the collection machine, where the listener was set up, you must specify the IP address and the listening port.

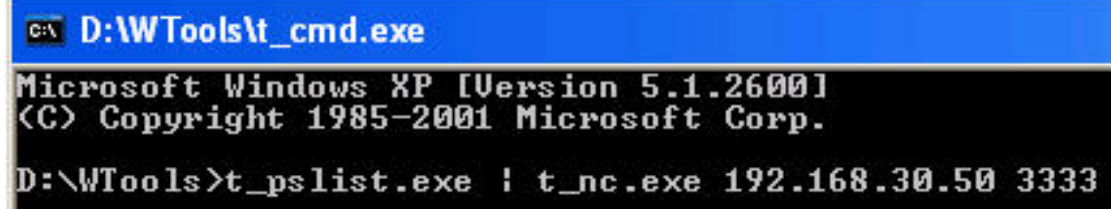

Figure 64: Using Trusted pslist and netcat to Specify IP Address and Listening Port

7. Look for suspicious processes by examining the results on the collection machine. To do this, type the following command and then use the up and down arrows to scroll through the results.

$$
\text { cat pslistText.txt | less }
$$

[rooteL i nuXWorkstation data]\# ls

pslist Text.t $x t$

[rooteLinu Workstation data]\# cat pslistText.txt iless_

\section{Figure 65: Looking for Suspicious Processes Using cat}

8. On this example machine, take notice of a process near the bottom of the list called tini.

Tini is a simple and very small ( $3 \mathrm{~kb})$ backdoor for Windows that listens on TCP port 7777 and provides a remote command prompt to anyone that connects. This process is suspicious enough for our purposes.

\begin{tabular}{|c|c|c|c|c|c|c|c|}
\hline ссAрр4 & 992 & 8 & 1 & 13 & 268 & И:Иย :ยИ.125 & ט:19:29.718 \\
\hline UMwareService & 1828 & 13 & 3 & 43 & 484 & 0:80:80.562 & 0:19:29.531 \\
\hline $\operatorname{tin} \mathrm{i}<$ & 1196 & 8 & 3 & 42 & 420 & В:80:80.156 & $0: 19: 26.593$ \\
\hline SpoolsSU & 1468 & 8 & 1 & 10 & 232 & ด:日ด:日ด.171 & $0: 19: 26.515$ \\
\hline suchost 1 & 1628 & 8 & 1 & 30 & 520 & В:80:80.109 & $0: 19: 20.359$ \\
\hline & & & & & & $n \cdot n a \cdot n a=$ & $n \cdot 10$ \\
\hline
\end{tabular}

\section{Figure 66: Suspicious Process Found}

9. Next, we need to locate where the executable for the process is located. On the collection machine, set up a netcat listener and send the results to a text file (tiniInfo.txt) by typing the command below.

nc $-1-p 4444>$ tinilnfo.txt

Again, any port above the reserved ports can be selected to set up a listener. This session will listen on port 4444 and send the results to the file tiniInfo.txt. 
Figure 67: netcat Command to Listen on Port 4444

10. From the target machine, send the data to the collection machine using the following command.

t_listdlls.exe -d tini | t_nc 192.168.30.50 4444

These tools are run from the resource CD of trusted tools and not the local machine. Specify the machine that has the netcat listener set up and what port it is listening on.

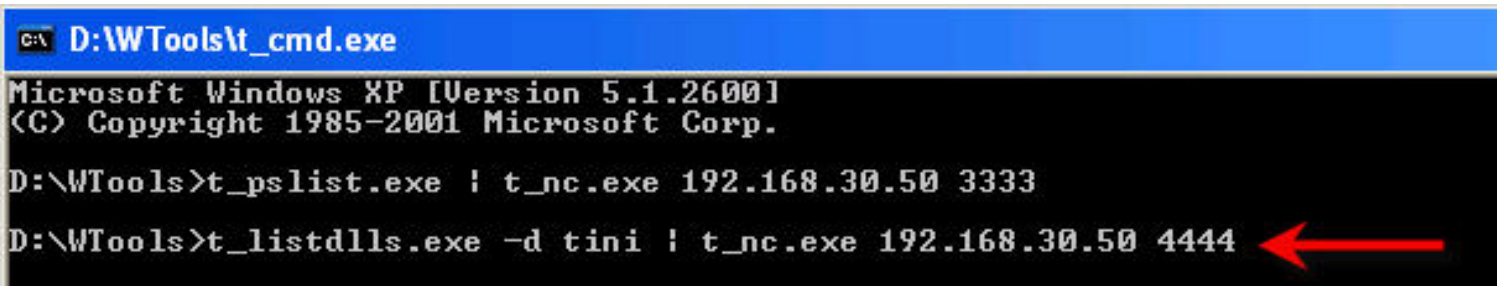

Figure 68: Specifying netcat Listener Machine and Port

11. To view the tiniInfo.txt file with the results from the trusted ListDLL command run on the target machine, use the command below from the collection machine. As you can see, tini.exe is located at $c$ :Iwindows|system32|tini.exe.

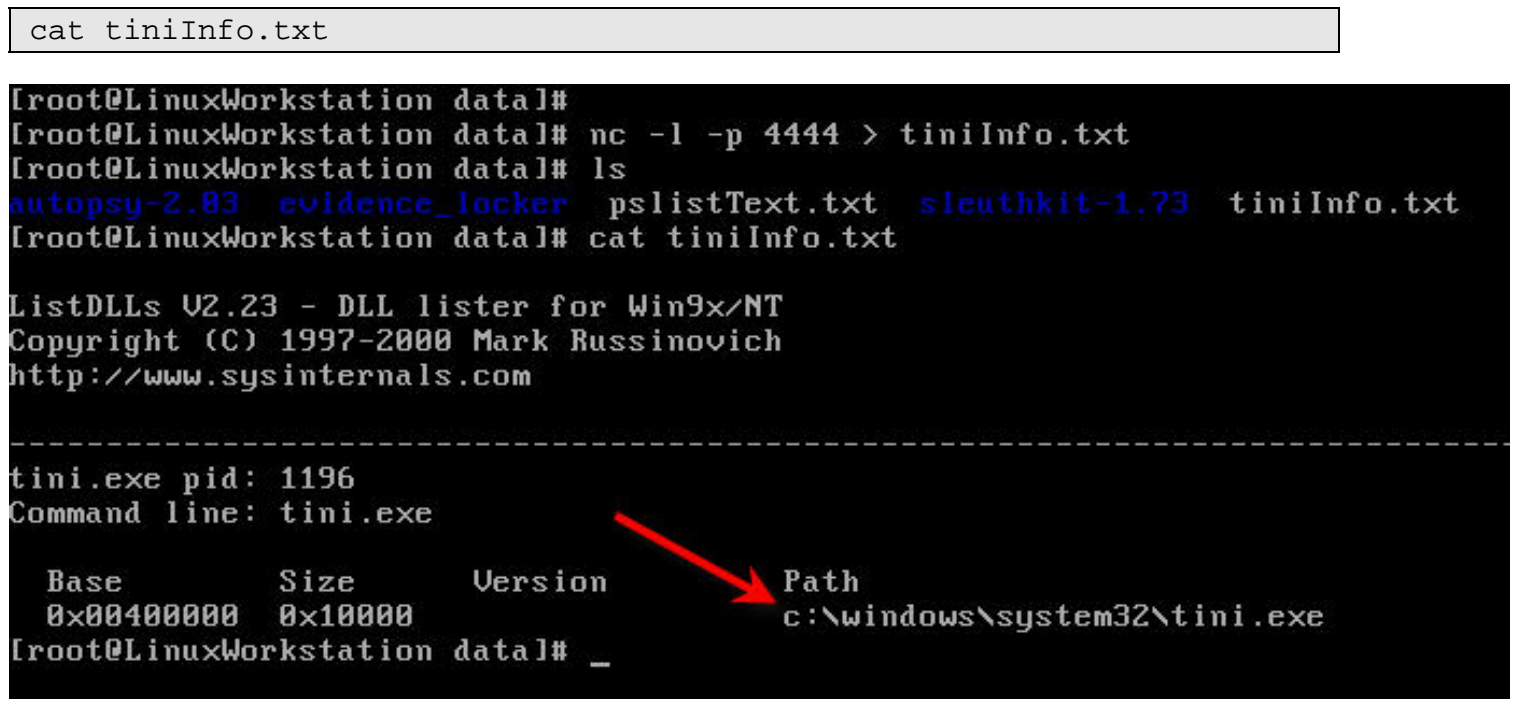

Figure 69: Viewing Path to a Suspicious Process

12. Now that we know the path to the suspicious process, we are ready to collect it. On the collection machine, enter the following to set up a netcat listening session, selecting a new port and file to write the capture as previously discussed.

nc -1 -p $5555>$ capturedTINI 


\section{Figure 70: Setting Up a Listening Session on a Suspicious Process}

13. On the target machine, using the trusted tools, enter the command below to copy the executable and send it to the collection machine.

t_dd.exe if= c: \windows \system32\tini.exe bs=512 | t_nc

192.168 .30 .505555

D: $\backslash W T o o l s>t$ _dd_exe if =c $: \backslash w i n d o w s \backslash s y s t e m 32 \backslash t$ ini.exe bs=512 i $t$ nc .exe 192.168.30.

505555

Forensic Acquisition Utilities, 1, 0, 0, 1035

dd, $3,16,2,1035$

Copyright (C) 2002-2004 George M. Garner Jr.

Command Line: $t$ _dd .exe if $=c: \backslash w i n d o w s \backslash s y s t e m 32 \backslash t$ ini.exe $b s=512$

Based on original version developed by Paul Rubin. David Mackenzie, and Stuart

emp

Microsoft Windows: Uersion 5.1 (Build 2600.Professional Service Pack 2)

04/03/2095 18:42:48 (UTC)

04/03/2005 13:42:48 (local time)

Current User: XPCOMPROMISEDUM \Student System

Copying $c: \backslash w i n d o w s \backslash s y s t e m 32 \backslash t$ ini.exe to CONOUT\$...

+0 records in

+0 records out

Figure 71: Collecting the Executable of a Suspicious Process

14. Once this is done, return to the collection machine and calculate a hash of the captured process. This will allow you to verify the integrity of any copies made for the purpose of analysis.

md5sum collectedTINI > tini.md5

[rooteL inuxWorkstation data]\# md5sum capturedTINI.dd > tini.md5_

Figure 72: Calculating a Hash of a Captured Process 


\title{
Linux System
}

\author{
The same ... but different
}

- List the processes

- Identify suspicious processes

- Copy process to remote location for further analysis

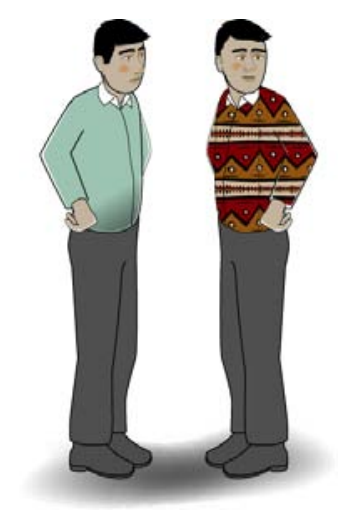

Running processes $p s-a u x$

Image file using $d d$

It is possible to leave a much smaller footprint on a Linux box than on a Windows machine. This is because the trusted tools on a Linux response disk can be completely self-contained. Without access to Windows source code, it is much more difficult to create completely selfcontained trusted tools.

When responding to a Linux system, the procedure is pretty much the same, unless the file that spawned the process has been deleted from the running system. The ps -aux command will list the running processes with associated binaries or the command-line arguments used to execute them. Once the location is enumerated, the $d d$ or $c p$ tools can be used to copy the file(s).

However, unlike Windows, a Linux user can delete the file used to launch a running process (and other files opened by the process) once the process has been executed and is running in memory. The file space will remain protected as long as the process continues to run and won't be overwritten. Retrieving the file data becomes significantly more complicated because the file associated with the process is no longer visible to ordinary file system tools, such as ls. If the process is selectively terminated or a reboot or shutdown occurs, then the file space will be marked as free and the process information may be lost from physical memory, as would be expected.

It is quite feasible to recover the data from a deleted file bound to a process, partly because the operating system has to protect the disk space while the process is running. While the file name is no longer visible in the directory structure, the inode that allocates the data space for the file is preserved until the process terminates. It's easy to search for files that have been deleted (or "unlinked") but are still protected by running processes. The command lsof $+a L 1$ 
will list all open files with an inode value of less than one, which is the case when they have been deleted. This command will display the inode and other metadata for the unlinked file(s). There are both commercial and open source tools that will take this value and recover the associated files. One such open source tool is icat, part of The Sleuth Kit, which is available at http://www.sleuthkit.org.

Collecting such information is not an overly complex process; however, it does exceed the scope of this handbook. This topic, "Recovering a Deleted Running Process in Linux," will be dealt with in a separate security improvement module (SIM). 


\section{Summary}

\section{Have response tools ready before they are}

needed

\section{Be familiar with processes normal to the hosts on your network}

\section{Leave as small a footprint as possible during collection}

There are a few things that can be done to significantly increase the chances of successfully identifying and extracting suspicious processes from a live system. First, have the tools built and tested before they are needed. Second, have a list of processes that normally run on a system. It is much more effective to compare running processes against a list of expected processes than to rely on a gut feeling regarding what is "normal." Finally, leave the smallest possible footprint while performing a capture. 


\section{Module 5: Understanding Spoofed Email}

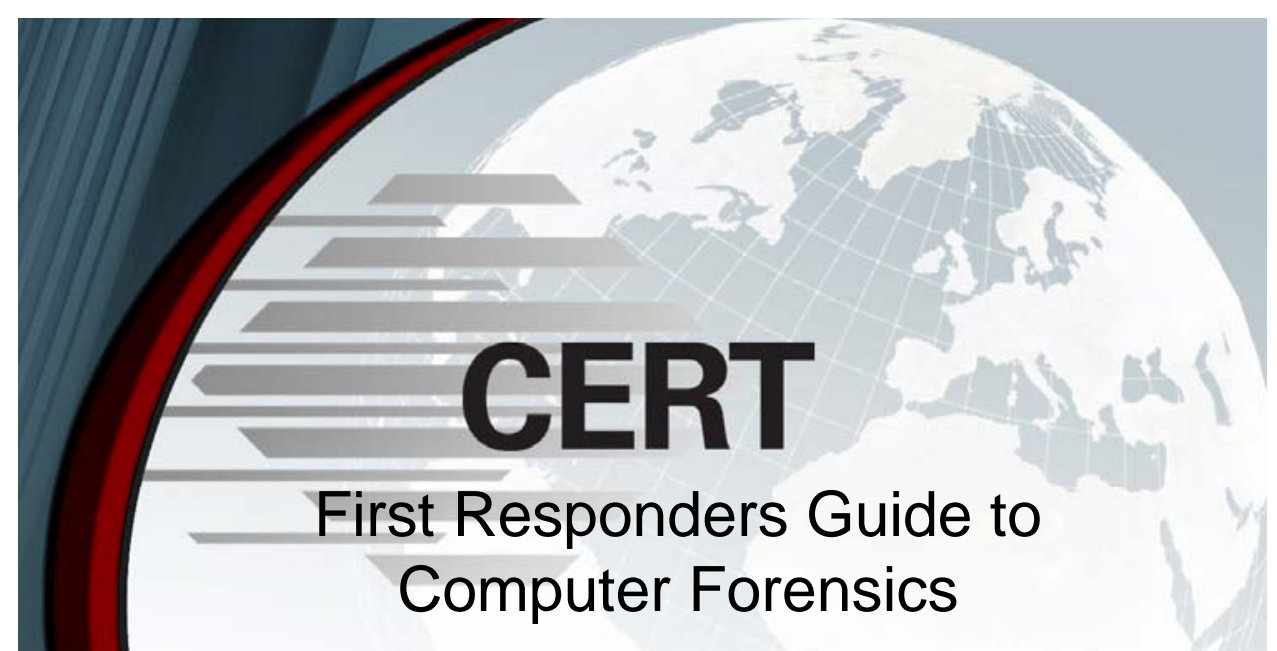

Module 5:

Understanding Spoofed Email 


\section{Objectives}

1. Understand how email is sent and received

2. Be able to interpret email headers

3. Review how spoofed email is sent

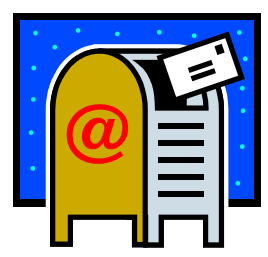

4. Learn to identify spoofed email

5. Tools and techniques for tracing spoofed email

\subsection{Objectives}

This module has five main objectives. First, it is important for individuals to understand how email is sent and received. Understanding the life cycle of an email and its back-end processes is the crux for being able to trace back spoofed messages. Second, individuals need to be able to interpret email headers. Doing so allows one to reconstruct the path an email message takes. Third, there are a variety of ways spoofed email can be sent, and it is important to keep them in mind when attempting to trace them back. The investigative approach being used may need to be adjusted depending on the spoofing technique. Fourth, email can be spoofed with great sophistication, and it is imperative that individuals are able to distinguish well-spoofed messages from legitimate ones. Fifth, there are numerous tools and techniques that can be used to trace the origins of a spoofed email message. Understanding the purposes of each will enable a person to potentially harvest a great deal of information from a spoofed email regarding the true sender. 


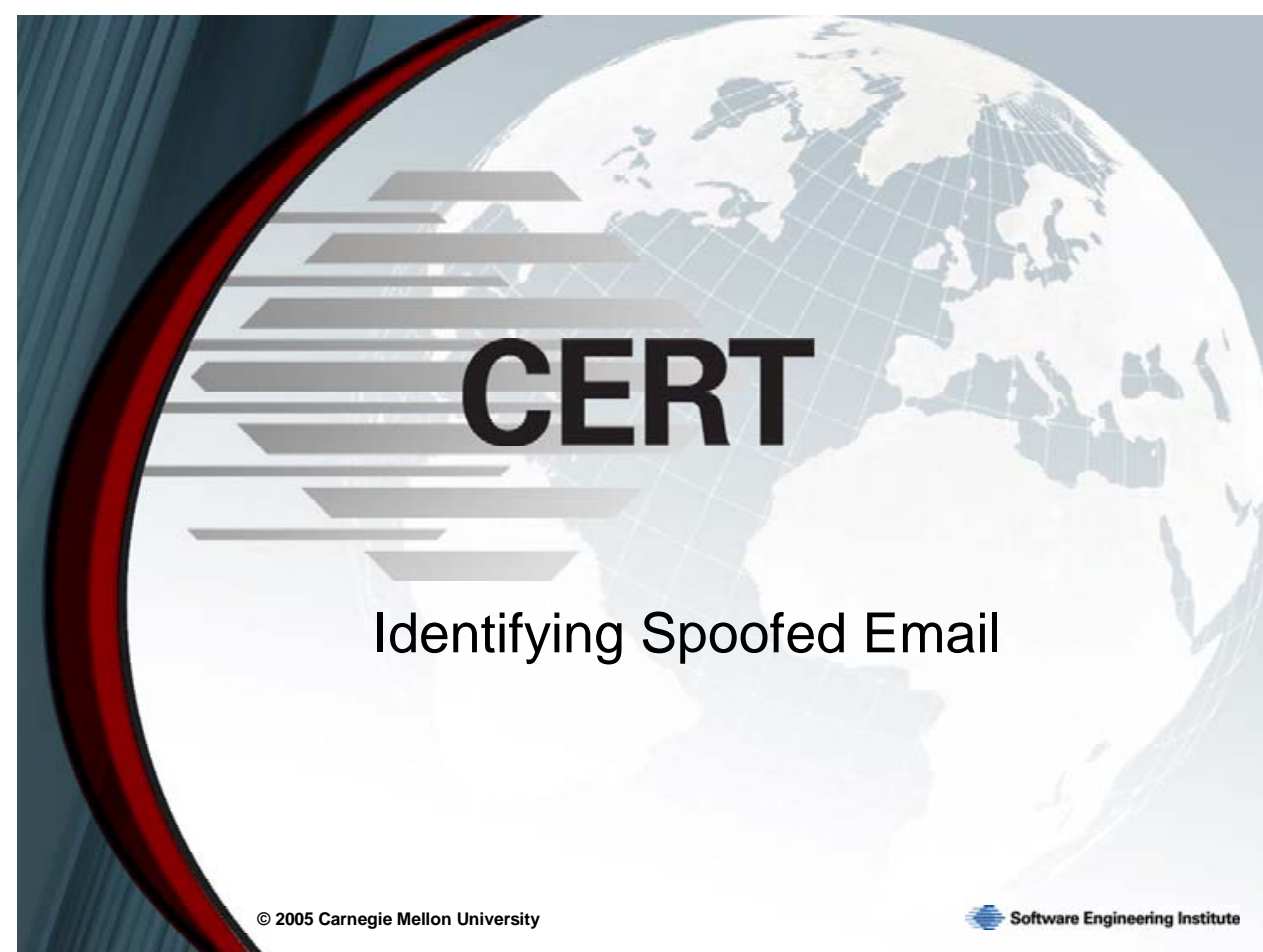

\subsection{Identifying Spoofed Email}




\section{The Threats}

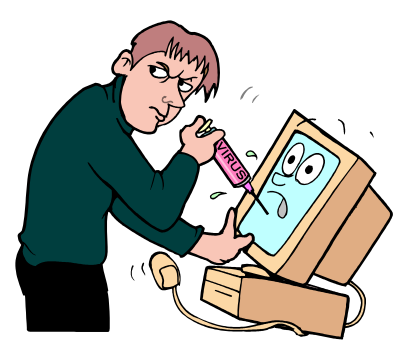

Criminals

Phishing schemes and deception to extort money and personal information

\section{Attackers \\ Viruses, Trojans, and worms propagated via email}

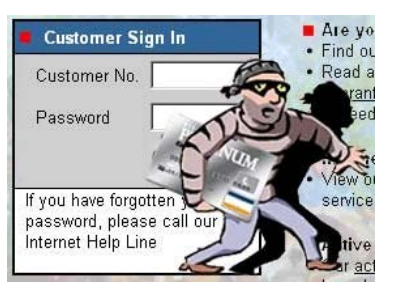

\subsubsection{Definition of the Problem}

Spoofed email has become a part of the daily messages that are delivered to a person's inbox. Spam has become a profitable enterprise for overzealous marketers and is a point of contention for those who receive it. However, spoofed email has become more than just a nuisance; it is a viable security threat to individual home users, organizations, and businesses. Attackers use spoofed email messages to propagate viruses, Trojans, and worms. Criminals use them for phishing schemes that attempt to extort money and information from unsuspecting users. Due to the lack of authentication in SMTP (Simple Mail Transfer Protocol), attackers and spammers can easily obfuscate their tracks and make it difficult to trace the origin of their email. 


\section{The Life Cycle of an Email 1}

\section{Alice wants to send an email to Bob}

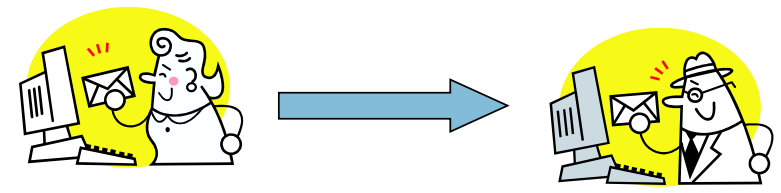

Four computers involved

1. alice.alphanet.com (Alice's computer)

2. smtp.alphanet.com (Alice's mail server)

3. mailhost.betanet.com (Bob's mail server)

4. bob.betanet.com (Bob's computer)

\subsubsection{Understanding the Process of Sending and Receiving Email}

\subsubsection{The Life Cycle of an Email}

Before attempting to identify the path of an email, one must first understand its life cycle. This topic will outline the order of events from the composition of an email all the way to its delivery to the receiver. For this example we are assuming that the email is legitimate and that it is being sent outside of the sender's network.

Typically, an email is handled by a minimum of four separate computers: the computer it is sent from, the mail server of the sender, the mail server of the receiver, and the computer that receives the email. Assume that Alice wants to send an email to her friend Bob. Alice and Bob use different Internet service providers for sending and receiving email. Alice uses alphanet.com and Bob uses betanet.com. The first thing that Alice does is compose an email on her computer, which we will call alice.alphanet.com. When Alice completes the message, she instructs her email client to send the message. At this point, her computer, alice.alphanet.com, sends the email to her mail server, smtp.alphanet.com. When smtp.alphanet.com sees that the message is to be delivered to someone in the betanet.com domain, it sends the message to betanet's mail server, mailhost.betanet.com. Mailhost.betanet.com knows that the message is for Bob and places it in his inbox. The next time Bob checks his email, Alice's message is delivered to him. 
Table 10: The Life Cycle of an Email

\begin{tabular}{|l|l|}
\hline Step 1: & Message is composed by Alice on her computer, alice.alphanet.com \\
\hline Step 2: & alice.alphanet.com sends the email to smtp.alphanet.com \\
\hline Step 3: & smtp.alphanet.com sends the email to Bob's email server, mailhost.betanet.com* \\
\hline Step 4: & Bob uses his computer, bob.betanet.com, to check his email \\
\hline Step 5: & bob.betanet.com retrieves Alice's email from mailhost.betanet.com \\
\hline
\end{tabular}

* At this point smtp.alphanet.com may not know the mail server it needs to contact; rather it may only know that it needs to send an email to someone within the betanet.com domain. In this case, smtp.alphanet.com will perform a DNS query in order to find the mail server for betanet.com.

Figure 73 depicts the life cycle of Alice’s email to Bob.

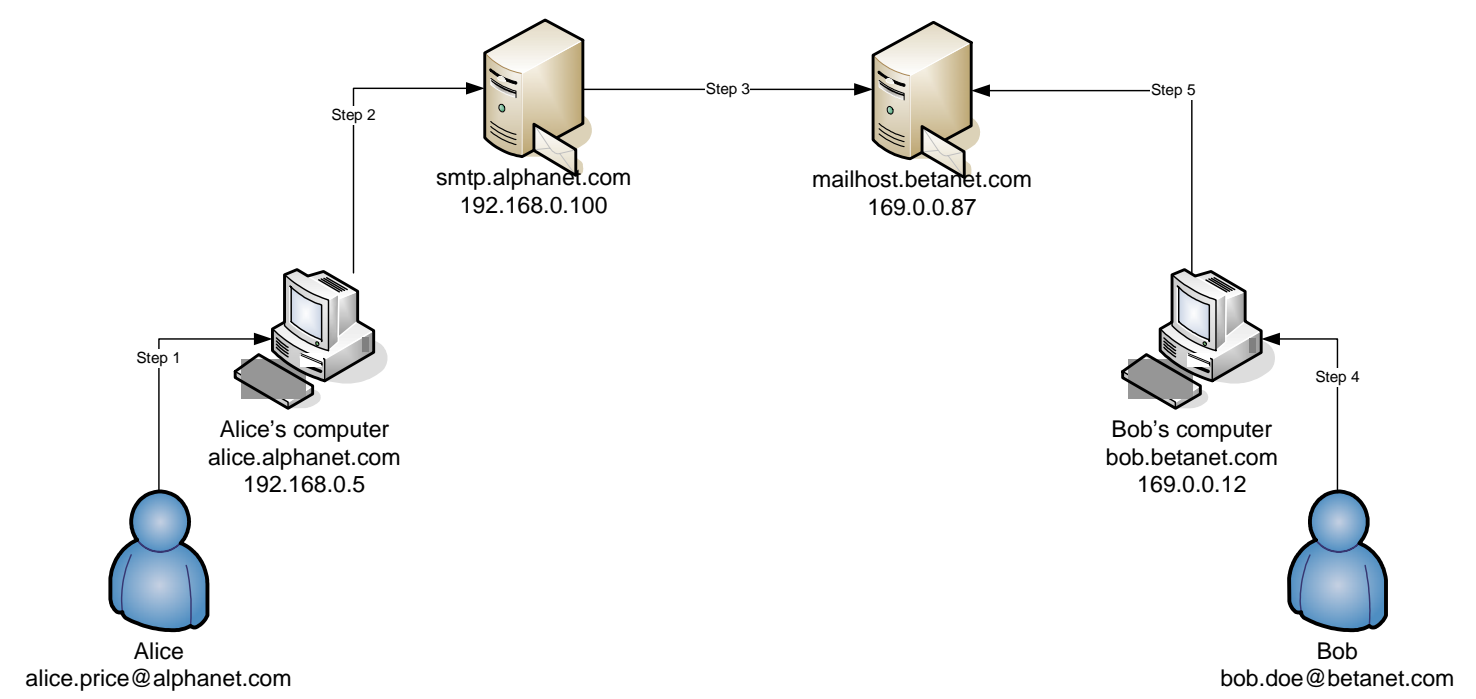

Figure 73: The Life Cycle of an Email 


\section{Overview of SMTP}

Simple Mail Transfer Protocol

Developed in the early 1980 s

RFC 821, 2821

Acts as a push protocol

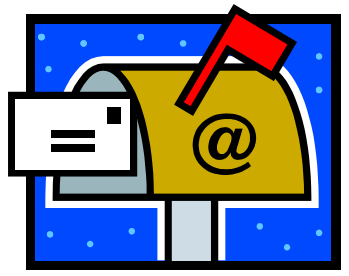

Other protocols needed to retrieve email (POP, IMAP)

Requires a TCP connection on port 25

No Authentication!

2005 Carnegie Mellon University

\subsubsection{Overview of the Simple Mail Transfer Protocol}

During the life cycle of an email, the process of it being sent to a mail server is handled by the Simple Mail Transfer Protocol (SMTP). SMTP was developed in the early 1980s and is outlined in RFC 821, which subsequently was obsoleted by RFC 2821 [NWG 01]. SMTP acts as a push protocol and only performs email delivery. As a result, separate protocols such as POP (Post Office Protocol) and IMAP (Internet Message Access Protocol) are needed to retrieve email messages from mail servers [Wikipedia 05e]. For the scope of this module, knowledge of POP and IMAP are not needed.

At first glance it may seem more efficient for Alice's mail client to send the email directly to mailhost.betanet.com rather than through her mail server, smtp.alphanet.com. However, Alice's mail server is much better equipped at guaranteeing delivery. During instances of connectivity interruptions or temporary computer downtime, the mail server is able to queue the message for delivery. Also, in general one can assume that a services machine will have more reliable uptime than a user machine. Lastly, mail servers have better name resolution and error handling capabilities. ${ }^{18}$

Before an email can be delivered via SMTP the client (sending computer) must initiate a TCP connection on port 25 with the receiving mail server. Once this connection is established, the client will send a sequence of commands to the server to identify itself, specify the sender, specify the receiver, pass off the email, and end the SMTP session. Other SMTP commands

18 Carnegie Mellon Computing Services. Cyrus Technology Overview. http://asg.web.cmu.edu/cyrus/1994-techoverview.html (1994). 
exist, but it is not necessary to focus on them for this topic. For a complete listing and explanation of SMTP commands, refer to RFC 2821. 


\section{SMTP Commands}

Most common SMTP commands

- HELO: used by sending machine to identify itself

- MAIL: initiates a mail transaction and provides the sender's email address

- RCPT: specifies the email address of the recipient

- DATA: signifies the message portion of the email

- QUIT: signals the termination of an SMTP session

\subsection{The HELO Command}

Once the SMTP session is established, the mail server sends a 220 code ( $<$ domain $>$ Service ready) to signal that it is ready. At this point the client will send a HELO command. The client essentially uses the HELO command to identify itself to the mail server. For example, if alice.alphanet.com was sending an email to smtp.alphanet.com, its HELO command would be "HELO alice.alphanet.com." It is important to note that the identifying information is provided by the client and there is no process of authentication to ensure that the client is who it says it is. Today, most mail servers have tools that are capable of determining the client's identity and recording it in the email headers. If the mail server accepts the client's HELO command, it replies back with a 250 code (Requested mail action okay, completed).

\subsection{The MAIL Command}

The MAIL command is used to identify the sender's email address and initiate a mail transaction. In Step 2 of Figure 73, the command would appear as "MAIL FROM: alice.price@alphanet.com.” The mail server may or may not verify that the given address is valid. If the command is accepted, the server will reply with a 250 code.

\subsection{The RCPT Command}

The RCPT command is similar to the MAIL command in that it specifies the email address of the recipient. In Step 2 of Figure 73, the RCPT command would appear as "RCPT TO: bob.doe@betanet.com.” This command does not verify that the email address provided is valid. If the command is accepted, the server will reply with a 250 code. 


\subsection{The DATA Command}

The DATA command indicates that the client would like to transmit the message portion of the email to the mail server. If the mail server accepts this command, it responds with a code 354 (Start mail input; end with $<\mathrm{CRLF}>$. $<\mathrm{CRLF}>$ ). The client signals the end of the email by placing a “.” on a line of its own. If the command is accepted, the server will reply with a 250 code.

\subsection{The QUIT Command}

When the client wishes to terminate its SMTP session with a mail server, it issues a QUIT command.

\subsection{SMTP Sequence of Figure 73, The Life Cycle of an Email}

In Step 2 of Figure 73 the client, alice.alphanet.com, needs to use SMTP to deliver the email to the mail server smtp.alphanet.com. An SMTP transaction for this step is illustrated with client commands in bold [Lucke 04]:

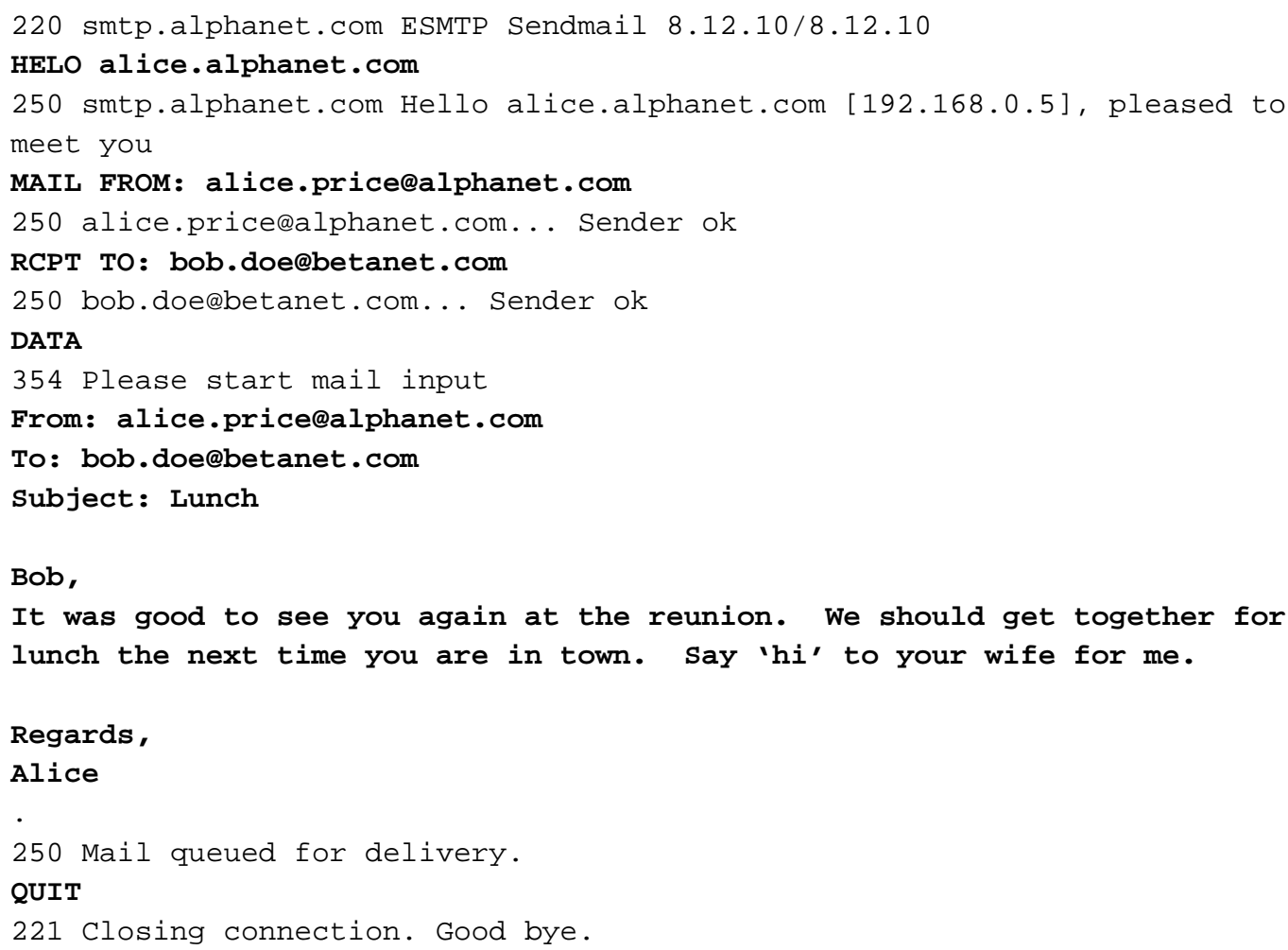




\section{Sample Email Headers}

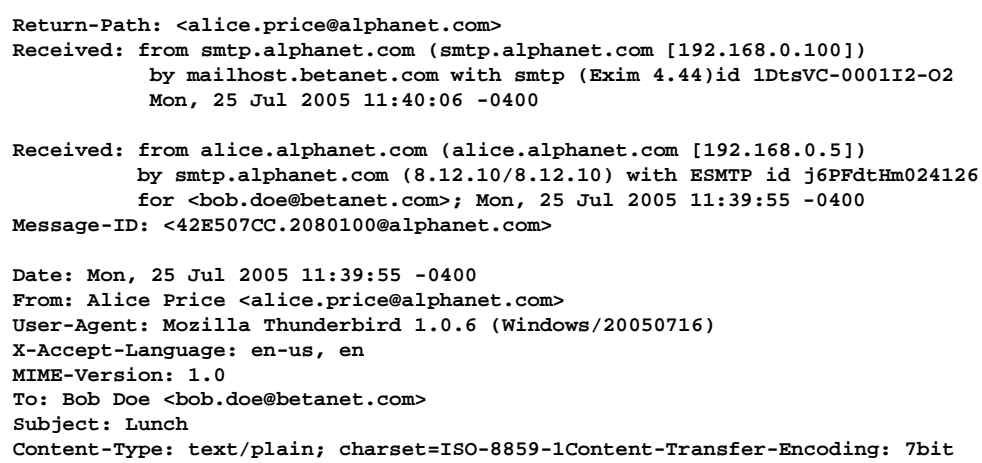

\subsubsection{Understanding Email Headers}

During the life cycle of an email, headers are added when the email is handled by different parties. In Figure 73 headers would be added in at the time of composition, at the alphanet.com mail server and betanet.com mail server. These headers contain information regarding the computers that handle a particular email. Being able to interpret these headers is an essential component to identifying and tracing spoofed email. In an email all header names are appended by a “:”.

\subsubsection{Interpreting Email Headers}

Mail clients by default usually do not display the full headers of a message. Usually there is an option to enable the display of all the headers or to view the message source. For example, in Mozilla Thunderbird one can display the full headers through the menu option View $\rightarrow$ Headers $\rightarrow$ All. One can also view the headers by displaying the message source: View $\rightarrow$ Message Source. It is important to note that not all mail headers are identical. The exact formatting and amount of information provided depends on the configurations used by the mail clients and mail servers involved.

Assume that Bob received the email sent by Alice in Section 5.2.2.2.6. Displaying all the headers of that message would produce the following: 
Table 11: Email Headers

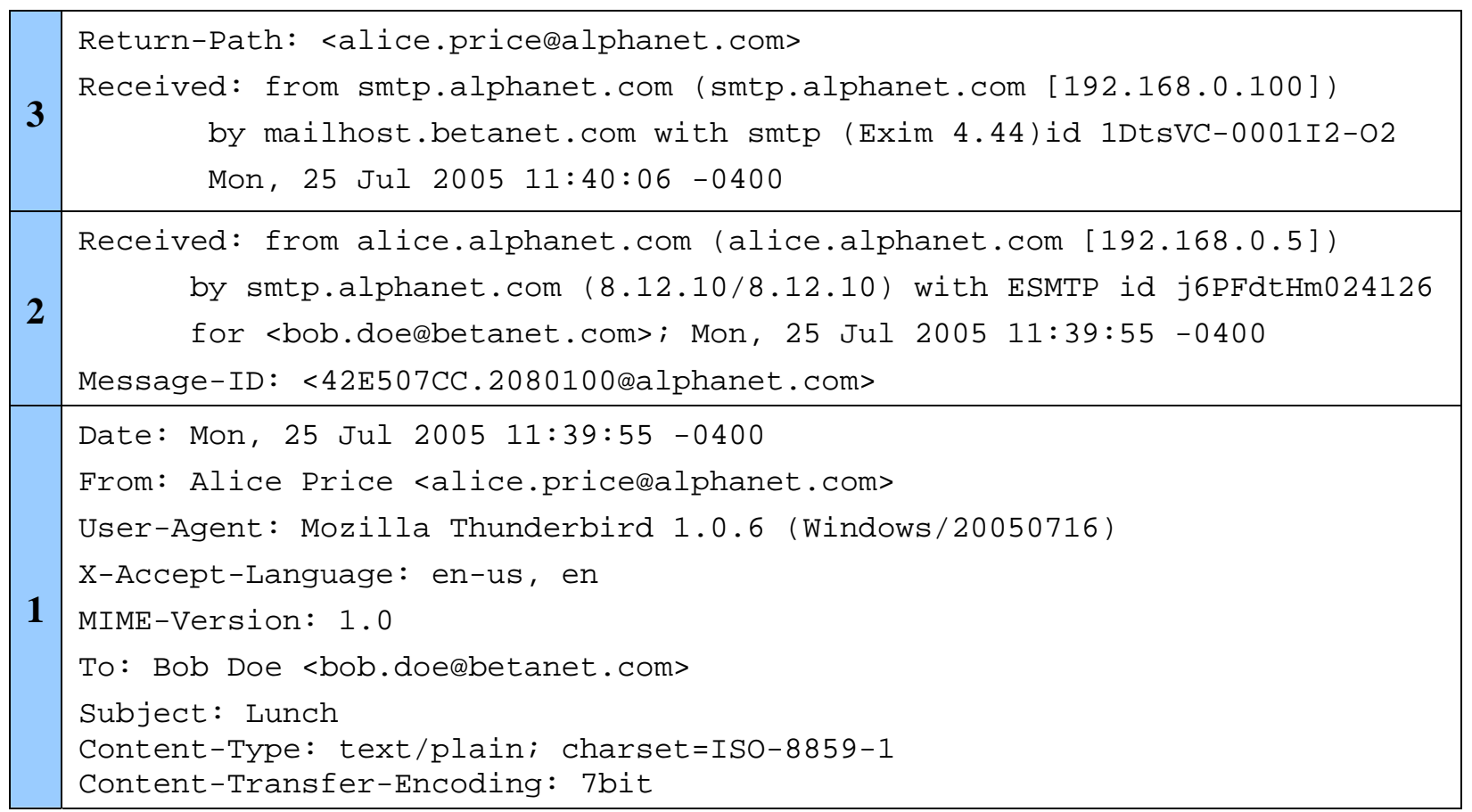

The first thing to understand about mail headers is that they are written from the bottom up. New headers are always written on top of the existing headers. In this example Alice's mail client wrote the first set of messages, Alice's SMTP server wrote the second set, and Bob's mail server wrote the third set.

\subsection{Headers from the Client}

Most of the headers added by the client such as "From:”, “To:”, and "Subject:” are selfexplanatory. The "Date:" header in this section signifies the time the email was composed. The rest of the headers can be interpreted as follows:

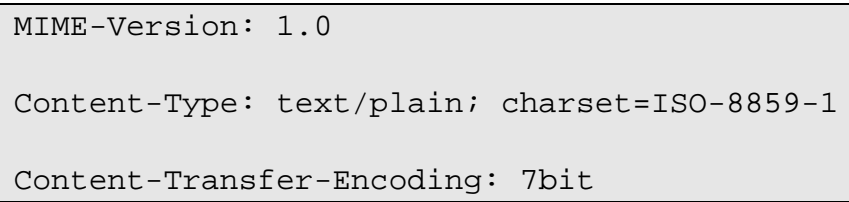

The email is in plain text using the ISO-8859-1 character set with 7-bit message encoding. 
This is an X-header, which is a non-standard header that provides additional information. The "X-Accept-Language" header informs the receiving server that email should be sent back in English. ${ }^{19}$

User-Agent: Mozilla Thunderbird 1.0.6 (Windows/20050716)

Alice used the Windows version of Mozilla Thunderbird v1.0.6 as her mail client.

\subsection{Headers from smtp.alphanet.com}

Once Alice finishes composing the email, her mail client sends it to her SMTP server, which in turn adds additional headers to the email. These headers can be found in section 2 of Table 11. Since the "Received" header consists of many components, it will be broken down line by line for better understanding.

Received: from alice.alphanet.com (alice.alphanet.com [192.168.0.5])

This message was received from a computer claiming to be alice.alphanet.com. The receiving machine determined that the sending machine's fully qualified domain name (FQDN) is alice.alphanet.com and its IP address is 192.168.0.5.

by smtp.alphanet.com (8.12.10/8.12.10) with ESMTP id j6PFdtHm024126

This message was received by smtp.alphanet.com, which is running Sendmail version 8.12.10/8.12.10. The message was assigned an ID of j6PFdtHm024126 by smtp.alphanet.com.

for <bob.doe@betanet.com>; Mon, 25 Jul 2005 11:39:55 -

The message is for bob.doe@betanet.com and was received on Monday, July 25, 2005, at 11:39:55 EST (Eastern Standard Time is -0400 GMT during daylight saving time).

Message-ID: <42E507CC.2080100@alphanet.com>

The Message-ID is a unique identifier that is assigned to each message. This is usually performed by the first mail server that handles the message. The first part of the ID is usually a unique string and the second part identifies the machine that assigned the ID. This is a universal ID, as opposed to the ESMTP or SMTP ID, which is specific to the receiving machine [Lucke 04].

\subsection{Headers from mailhost.betanet.com}

Once smtp.alphanet.com processes the email, it is sent to mailhost.betanet.com, where Bob will eventually retrieve the message.

19 The A3C Connection. Headers of a Legit Email Message. http://www.uic.edu/depts/accc/newsletter/adn29/legitmail.html\#Language (2000). 
Received: from smtp.alphanet.com (smtp.alphanet.com [192.168.0.100])

This message was received from a computer claiming to be smtp.alphanet.com. The receiving machine determined that the sending machine's FQDN is smtp.alphanet.com and its IP address is 192.168.0.100.

by mailhost.betanet.com with smtp (Exim 4.44)id 1DtsVC-0001I2-02

This message was received by the computer mailhost.betanet.com, which is running Exim version 4.44. The receiving mail server, mailhost.betanet.com, assigned this message an ID of 1DtsVC-0001I2-O2 for its own records.

Mon, 25 Jul 2005 11:40:๑6 $-\odot 4 \odot \odot$

The mail server mailhost.betanet.com received this message on July 25, 2005 at 11:40:06 EST. Notice that the timestamps in the headers are in chronological order. This will help later in trying to distinguish between real headers and fake headers.

Return-Path: <alice.price@alphanet.com>

Any replies to this email should be sent to the address alice.price@alphanet.com. The "Return-Path" header is written by the SMTP server that makes the final delivery. The address in this header is the address that was provided in the MAIL command. 


\section{How Spoofed Email Is Sent}

\section{Open relays}

Compromised machines

Self-owned mail servers

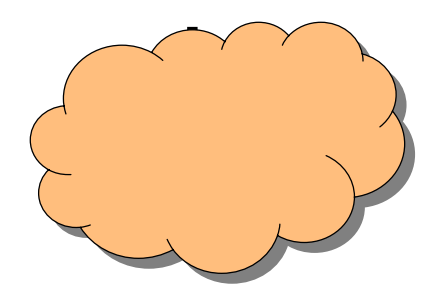

Temporary accounts

Hijacked accounts

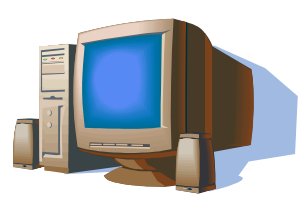

\subsubsection{How Spoofed Email Is Sent}

\subsubsection{Open Mail Relay}

An open mail relay refers to a mail transfer agent that will deliver mail for any sender, regardless of who it is. Up until the late 1980s, email was not delivered directly from the sender to the receiver. Instead, routes were set up where the messages would be relayed from point to point [Lucke 04]. This paradigm allowed individuals to send messages through a mail server even if they were not valid users of the system. However, this model has become open to abuse by individuals attempting to mask their origin, such as criminals and unethical advertisers. The norm now is to allow only valid users of a system to send email from it.

As an example, in the email life-cycle diagram, pretend there is a third email server called smtp.gammanet.com. Alice is a valid user of the alphanet.com domain but not of the gammanet.com domain. As a result, Alice can send email only via smtp.alphanet.com but not smtp.gammanet.com (Figure 74). 


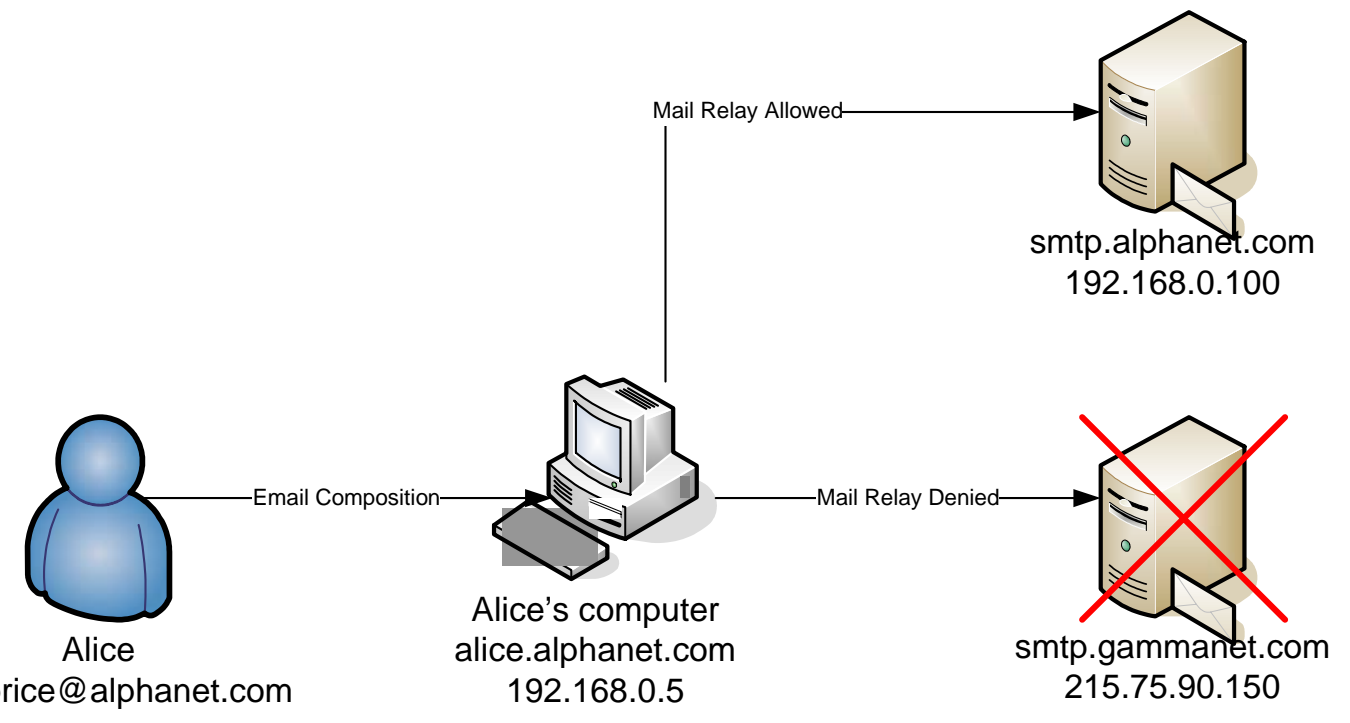

Figure 74: Mail Delivery for Valid Users

However, if smtp.gammanet.com was configured to be an open relay, Alice would not be prohibited from sending email via it. Since Alice is not a user in the gammanet.com domain and since she does not have to provide valid credentials (host identity and source email address), she can easily cover her tracks by sending email via smtp.gammanet.com. Figure 75 shows how Alice can easily spoof an email using an open relay.

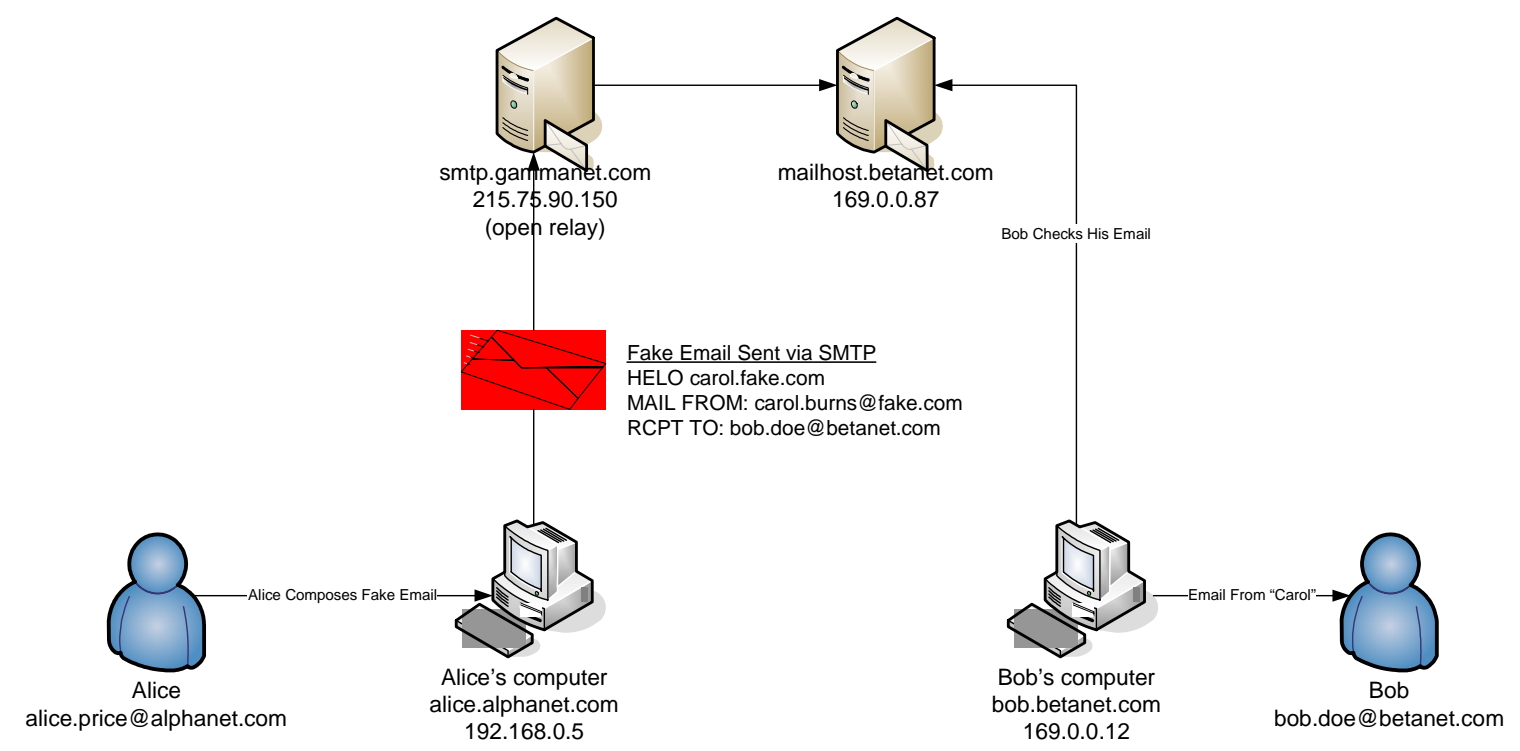

Figure 75: Spoofed Email via an Open Relay

Since most mail servers stamp the host's true identity (usually the IP address) in the email headers, Alice is not able to completely cover her trail, but it is more than enough to fool an unsuspecting recipient. 


\subsubsection{Compromised Machines}

One method that spammers have used in order to gain access to open relays is to compromise machines on the Internet. Often this is through the installation of a Trojan. These applications gained their name through the similarities with the Trojan horse from the Greek story of the Trojan War. Like the horse in the myth, a Trojan application may appear to be legitimate, but in reality it is a tool used by attackers to compromise a host. Often, Trojans will be posted to file-sharing networks named as legitimate files, enticing users to download them.

The Sobig worm and its variants were received via an email with an attachment. Once the attachment was opened, it would infect any computer running Microsoft Windows. The worm would then use the host computer to spread itself by sending email to other users or copying itself to any open network shares, and would also download a proxy application from the Internet. Once downloaded, the proxy would function as a mail relay listening on a non-standard port for incoming connections or would attempt to send out mail.

\subsubsection{Self-Owned Mail Servers}

Perhaps one of the easiest ways to send spoofed email is to set up one's own mail server. There are many programs that make it easy to do this. Some readily available programs include Sendmail, Postcast and QK. Through the use of these and similar applications, a person with a broadband Internet connection could send over 1,000,000 10-50KB email messages in one hour [Sendmail 05]. When spammers use this method they can add extra received headers to obfuscate the true path of the email. Adding extra headers to an email will make it appear as though the mail was actually sent from a machine other than the spammer's mail server.

\subsubsection{Temporary Accounts}

Another method that spammers use to send spoofed email is to create temporary mail accounts with ISPs. This can be done by using false credentials or stolen credit cards. The temporary account is used until the ISP cancels it for being used to send spam. Other forms of temporary email accounts include services such as Hotmail and Yahoo. Spammers have also been known to write scripts that sign up for multiple accounts and send spam automatically.

\subsubsection{Hijacked Accounts}

An alternative to using temporary accounts or compromised machines is hijacking valid user accounts. While this is not the most popular method for spammers, there have been recorded incidents of such activity. The benefit of using temporary or hijacked accounts is that they will often be able to bypass spam filters, since they have not been used for spam and appear to be legitimate. For example, in 2002 a spammer by the name of Charles Frye used a password cracking tool called WWWHack to hijack dozens of accounts and send millions of spam messages [McWilliams 05]. In 2005 Frye was sentenced to one year in jail and six years of probation. During that time he is not permitted to use a computer. 


\section{Identifying Spoofed Email}

The "Received" headers are crucial!

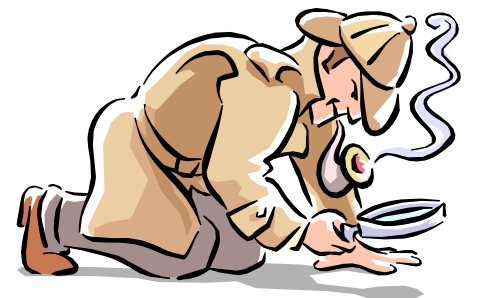

Received: from fusionse.com

(AMarigot-102-1-4-205.w81-248.abo.wanadoo.fr [81.248.108.205])

by mailhost@legitserver.com (8.12.10/8.12.10)

with SMTP id j6PFVvp5027789

for <realuser@legitserver.com>; Mon, 25 Jul 2005 11:31:58 -0400

\subsubsection{How to Identify Spoofed Email}

\subsubsection{Carefully Examine the "Received" Headers}

There are a number of telltale signs that may indicate an email is not legitimate. All of them involve interpreting a message's headers. One of the more resourceful and useful headers is the "Received" header. Sections 5.2.3.1.2 and 5.2.3.1.3 show that this header includes the sender's fully qualified domain name and/or its IP address. Additionally, the receiving computer determines the sender's IP address on its own and adds that information into the header.

Received: from smtp.alphanet.com (smtp.alphanet.com [192.168.0.100])

From sender via SMTP

HELO
Stamped from the receiving machine, mailhost.betanet.com

In a legitimate email the two addresses will match. However, if a sender provides invalid host information in the HELO command, it will be reflected in the "Received" header. The following "Received” header is from an actual spoofed email: 
Received: from fusionse.com

(AMarigot-102-1-4-205.w81-248.abo.wanadoo.fr [81.248.108.205])

by mailhost@legitserver.com (8.12.10/8.12.10)

with SMTP id j6PFVvp5027789

for <realuser@legitserver.com>; Mon, 25 Jul 2005 11:31:58 -

Notice that the sender claims to be fusionse.com but the receiving mail server determined that the sender really came from an ISP in France. In this instance, it is most likely that this email was sent from a compromised host. 


\section{Fake "Received" Headers}

\section{Always read headers from the top down}

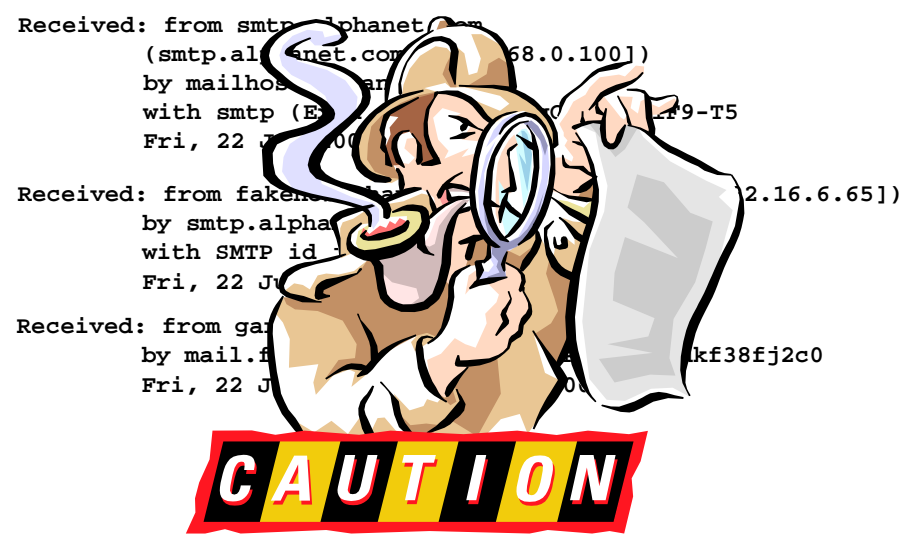

\subsubsection{Look Out for Spoofed Headers}

One technique that spammers and attackers use to cover up their tracks is to add bogus headers to a message. These headers are intended to confuse individuals attempting to trace an email's true origin. The best way not to be fooled by fake headers is to read email headers starting at the top. Since fake headers are added by the sender, they will always be beneath the real headers. Illustrated next is a set of headers from an email that contains a fake "Received" header:

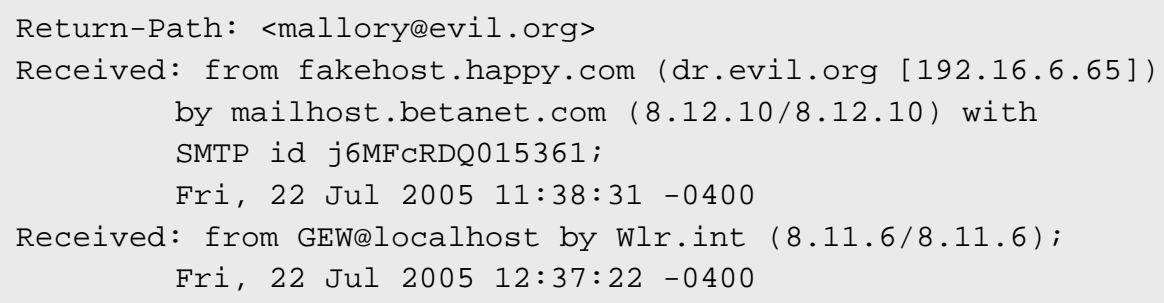

In this particular set of headers, it is fairly easy to spot the fake received line. First, by reading the headers from the top down one can see there is a discontinuous set of events from the top "Received" header and the bottom one. The bottom header does not explain how this message got to dr.evil.org. Second, the information in the bottom "Received" header does not appear to contain valid host addresses (GEW@localhost and Wlr.int). Third, the timestamp in the bottom and supposedly first header is almost an hour ahead of the timestamp in the top header. This could be attributed to an erroneous clock configuration, but because of the other red flags, it is more likely to be a fake header. 


\subsubsection{Comparing Timestamps}

A quick way to check the legitimacy of an email is to compare the timestamps in the "Received" headers and ensure that the chronology is reasonably accurate. Since the timestamps are written by the local, receiving machines, it is likely that they will not be perfectly in sync. A grossly misaligned timestamp may be an indication that an email is not legitimate. Fake "Received" headers will most likely contain timestamps that are out of line with the real timestamps. However, it is conceivable that a skewed timestamp is the result of bad clock configuration rather than an indicator of a spoofed email. 


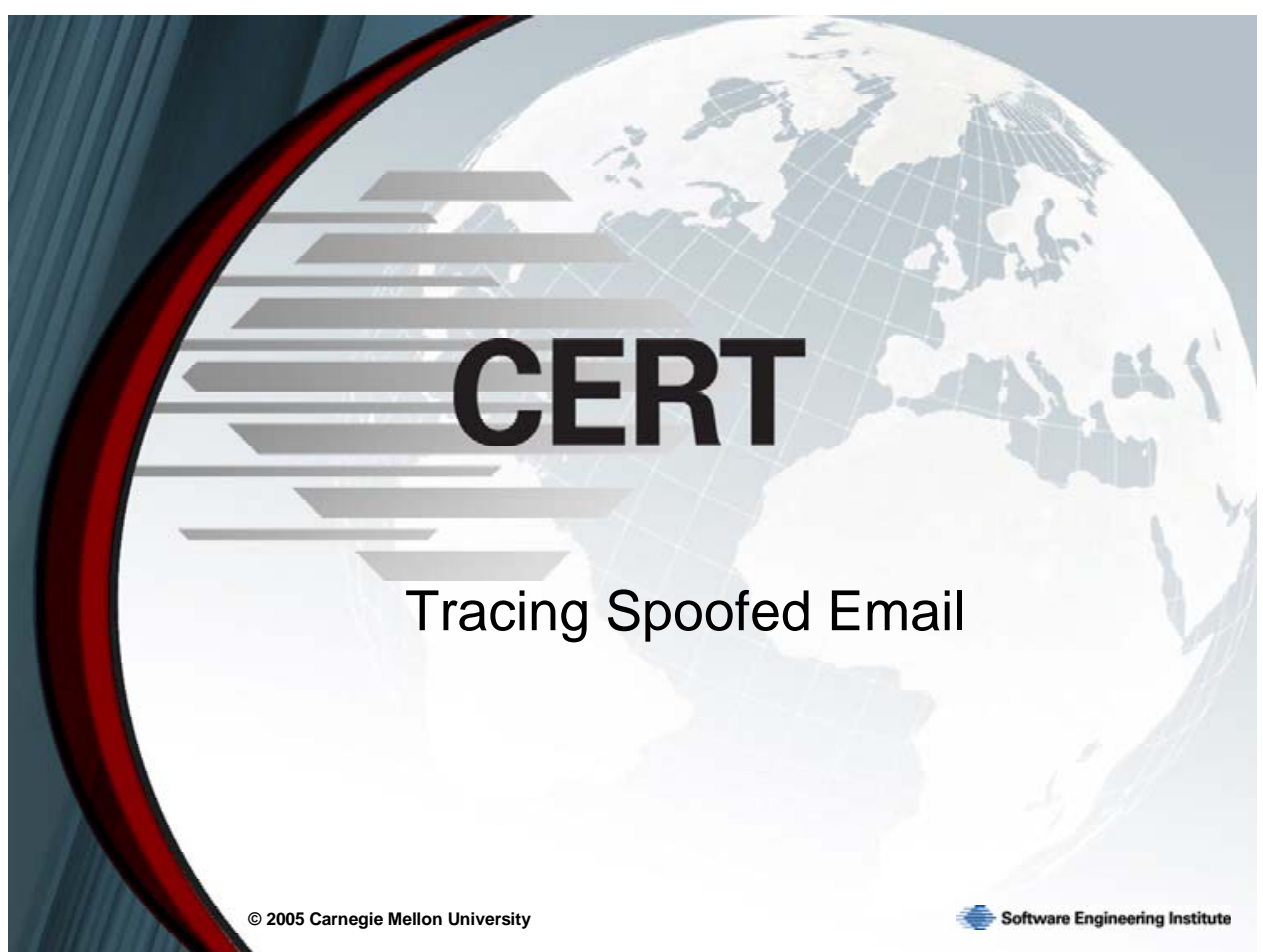




\section{Tracing Spoofed Email}

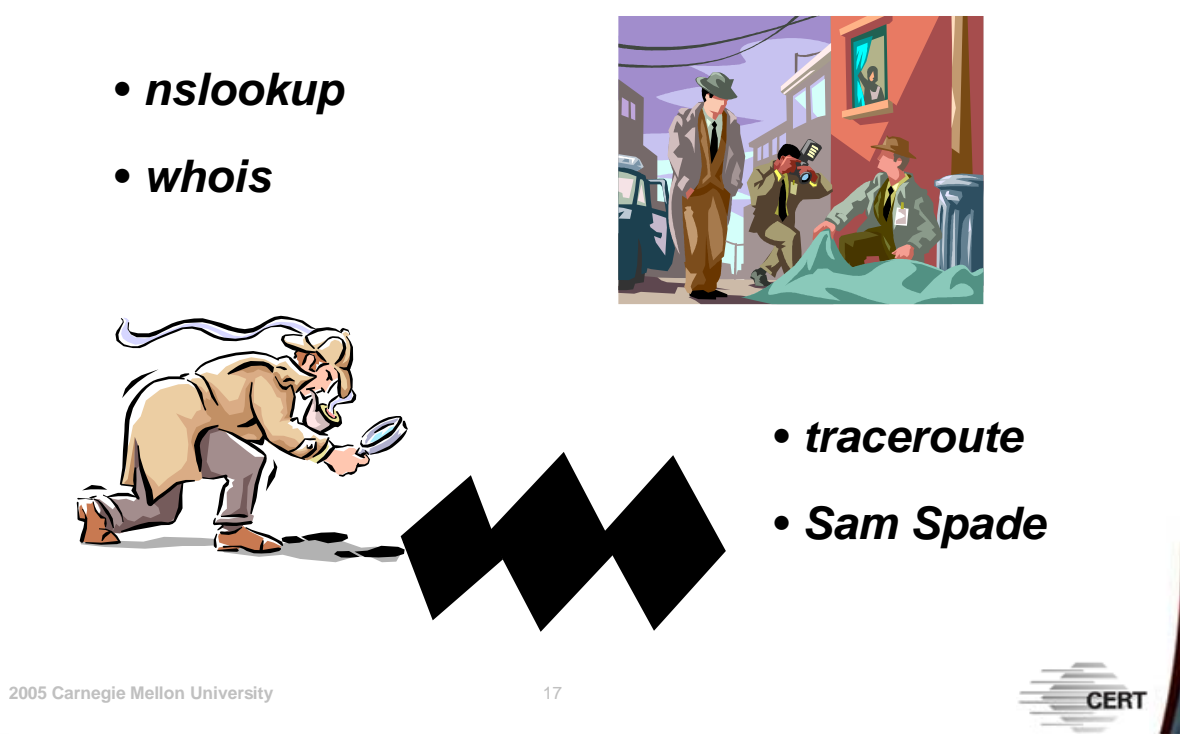

\subsection{Tracing the Origins of a Spoofed Email}

Once a spoofed email has been identified, the next step is to attempt to determine its origin. Tools such as nslookup, whois, traceroute, and Sam Spade can yield very useful information. These tools can help find information about the sending host, such as physical location, organizational affiliation, and contact information. It is possible that using these tools will not reveal the identity of the culprit; however, they will help one gather more information about the first known hop. One can then contact system/network administrators at that hop and attempt to gather further information using data from that hop's "Received" header, such as the message ID. It is important to note that even if one is able to identify the machine that sent a spoofed email, it may not be the end of the line. As has been explained in Section 5.2.4.2, many spoofed messages are sent from compromised computers. In this case, finding the true sender may involve performing forensics on the compromised machine. In this type of situation one may need to consult with legal counsel. 


\section{nslookup}

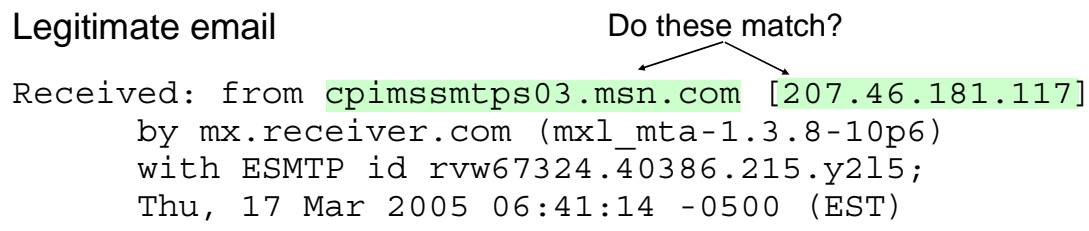

\section{Spoofed email}

Received: from cign.de ([221.153.24.156]) by mx.domain.com (8.12.10/8.12.10) with SMTP id q6S3NUK2958123 for<receiver@domain.com>; Wed, 27 Jul 2005 23:23:33 -0400

\subsection{1 nslookup}

The nslookup name stands for name server lookup. For the purposes of spoofed email, nslookup is used to perform reverse DNS lookups on IP addresses and vice versa. It is a useful utility for quickly verifying the host information contained in the "Received" headers from unreliable hops. As a rule of thumb, one cannot consider hops outside of one’s own domain and control to be trustworthy. The nslookup tool works by querying a name server with either the IP address or FQDN provided by the user. From a Windows command prompt, the syntax for nslookup is as follows:

\section{c: $\backslash>$ nslookup [computer to find] [name server]}

Providing the name server is optional. If a name server is not provided, nslookup will use the default name server, which is usually the name server for the domain that the querying machine is on. The default name server may not provide an authoritative answer. To get an authoritative answer, one may need to query the name server for the computer in question.

The following "Received” header is from a legitimate email. Notice that when an nslookup is performed, it matches the IP address recorded by the receiving mail server:

Received: from cpimssmtps03.msn.com [207.46.181.117]

by $\mathrm{mx}$.receiver.com (mxl_mta-1.3.8-10p6)

with ESMTP id rvw67324.40386.215.y215;

Thu, 17 Mar 2005 06:41:14 - 0500 (EST) 


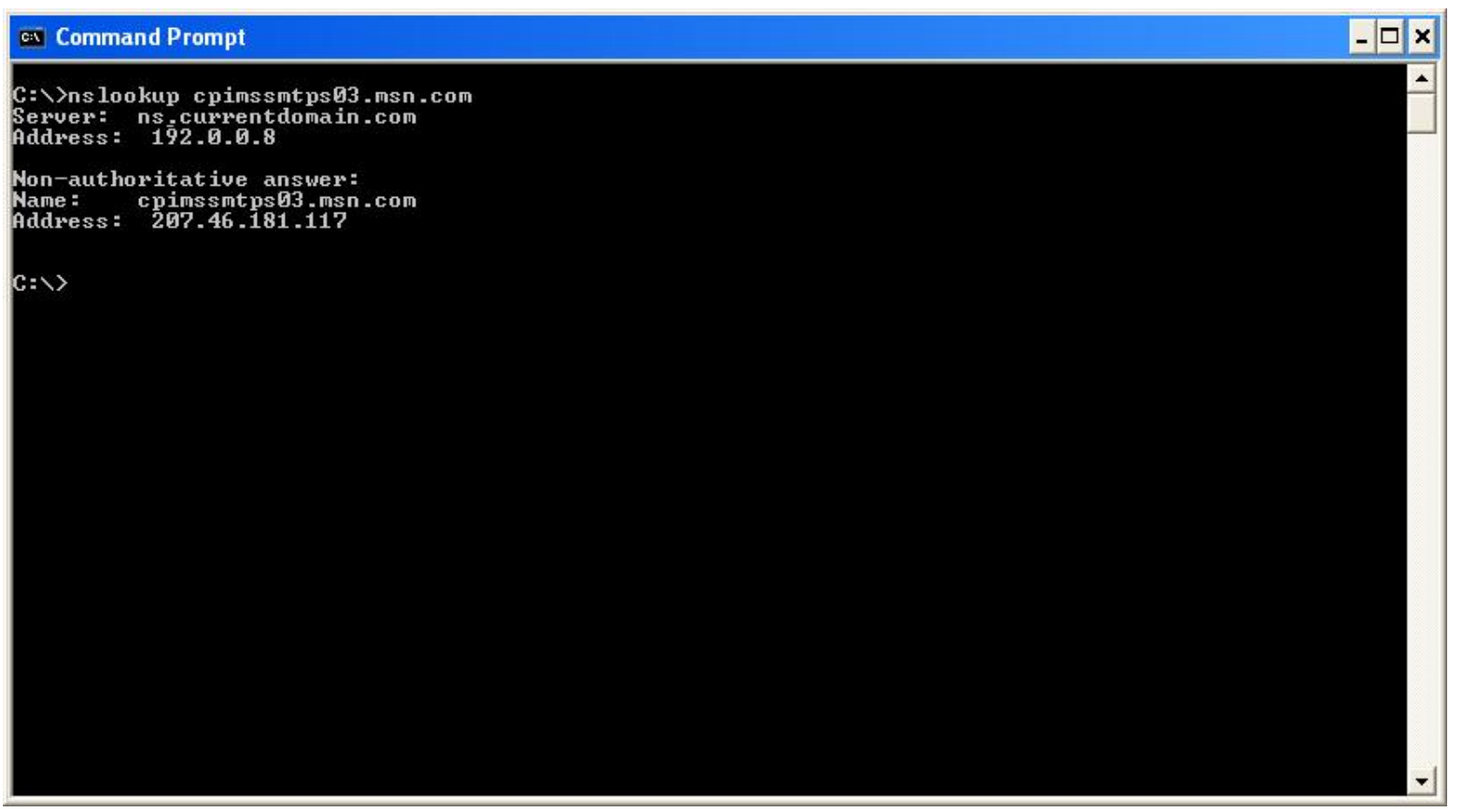

Figure 76: nslookup of Valid Fully Qualified Domain Name

The following "Received" header is from a spoofed email:

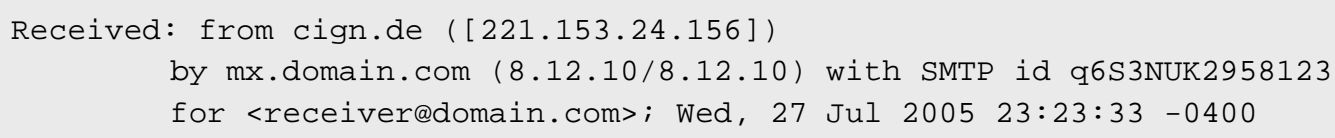

The sender claims to be from cign.de, but this information cannot be relied on. An nslookup can be used in this instance to see if the IP address for cign.de matches the IP address recorded by the receiving machine. The results from nslookup (Figure 77) show that the sender falsified the host information. The address for cign.de, 81.169.145.69, does not match the real address of the sender, which is 221.153.24.156. Now that the true identity of the sender has been discovered, nslookup can be used to attempt to find the host's fully qualified domain name. 


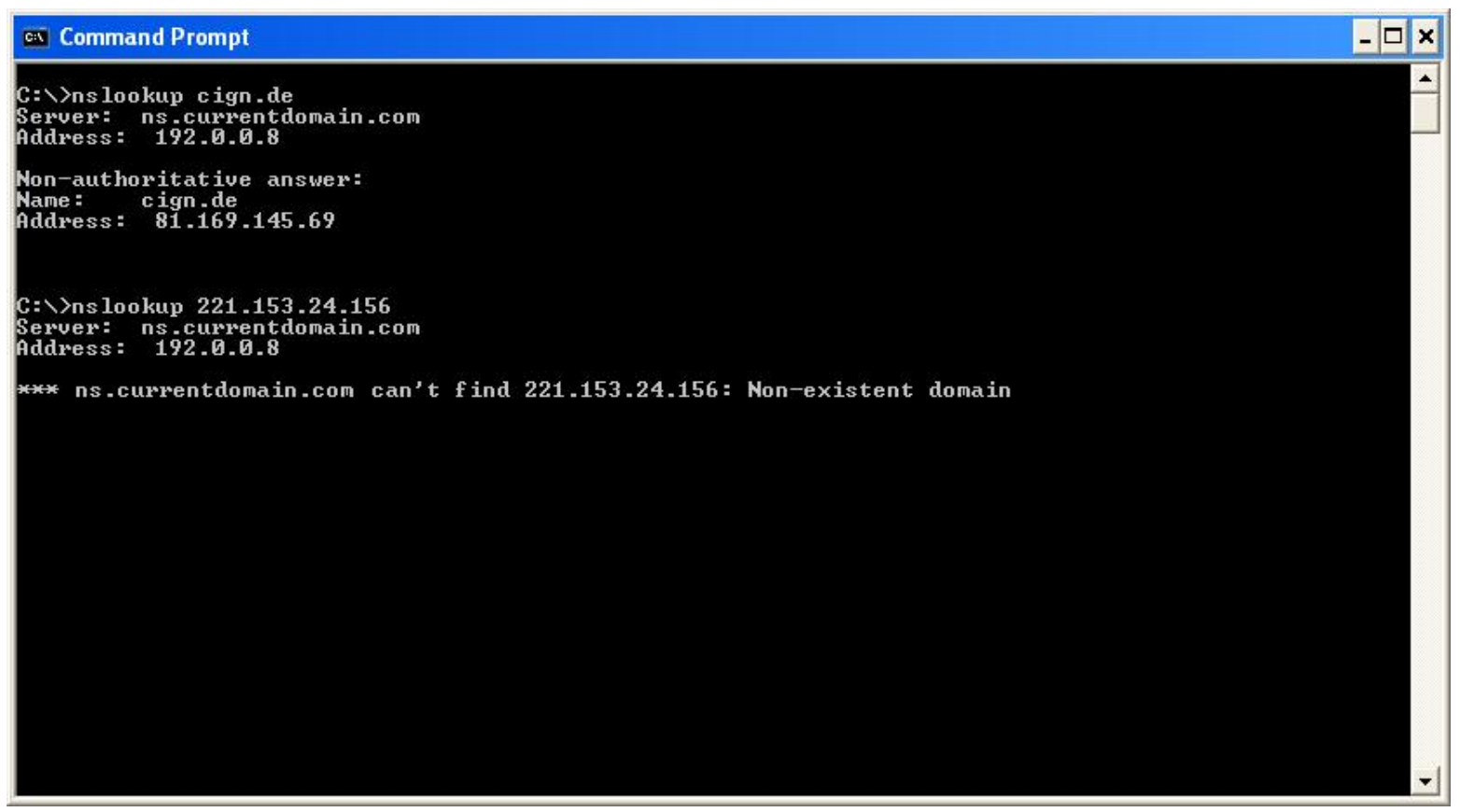

Figure 77: nslookup of Falsified Host Information

Figure 77 also shows that when nslookup is run on the IP address 221.153.24.156, the name server cannot find information about that particular IP. The name server used by nslookup will not always be able to provide information on a given IP address. In these instances it will be necessary to find out the owner of the IP block that contains the address in question. 


\section{Whois - Dual Purpose}

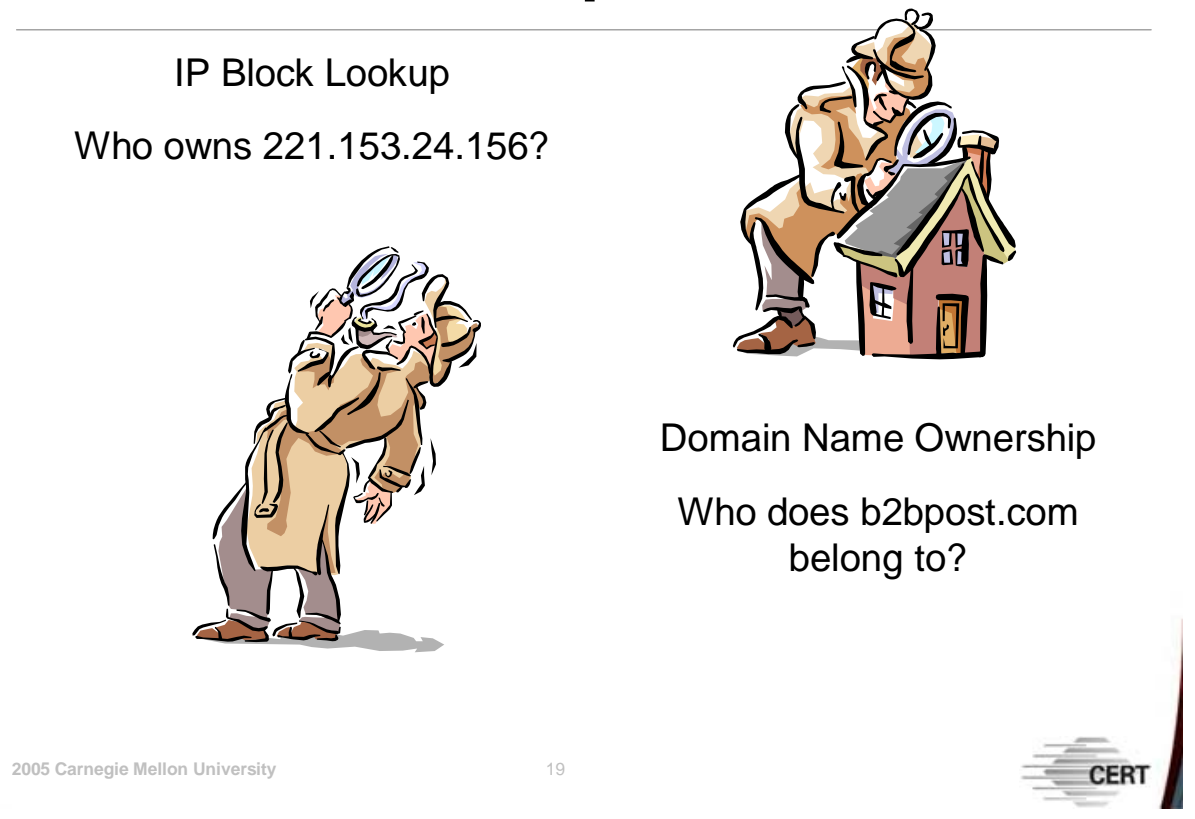

\subsection{2 whois}

The whois utility can be used to determine the owner of a particular IP block, as well as yield greater information on a domain such as location, contact information, and name servers. All of this information can be useful in tracking down the sender of a spoofed email. Whois is a command line utility that is available in the Linux environment but is not native to Windows. In order to use whois in a Windows environment, one will need to download a third-party utility. Section 5.3.4 briefly describes the Sam Spade tool for Windows, which incorporates whois. Whois queries can also be performed via websites such as samspade.org. However, it is important to consider that the information contained in the WHOIS databases may not be completely accurate; in fact, it is not uncommon for WHOIS contact information to be falsified.

\subsubsection{IP Block Identification}

In Section 5.3.1, nslookup failed to provide information regarding the IP address 221.153.24.156. Whois can be used to query the ARIN database (American Registry for Internet Numbers) to find out information about the IP address in question. ARIN is responsible for the registration and administration of IP addresses in Canada, the United States, and parts of the Caribbean [Wikipedia 05a]. They maintain a publicly accessible database that contains ownership information about IP blocks within their geographic domain. To query the ARIN database in a Linux environment, the following command is used: whois 221.153.24.156@whois.arin.net. 


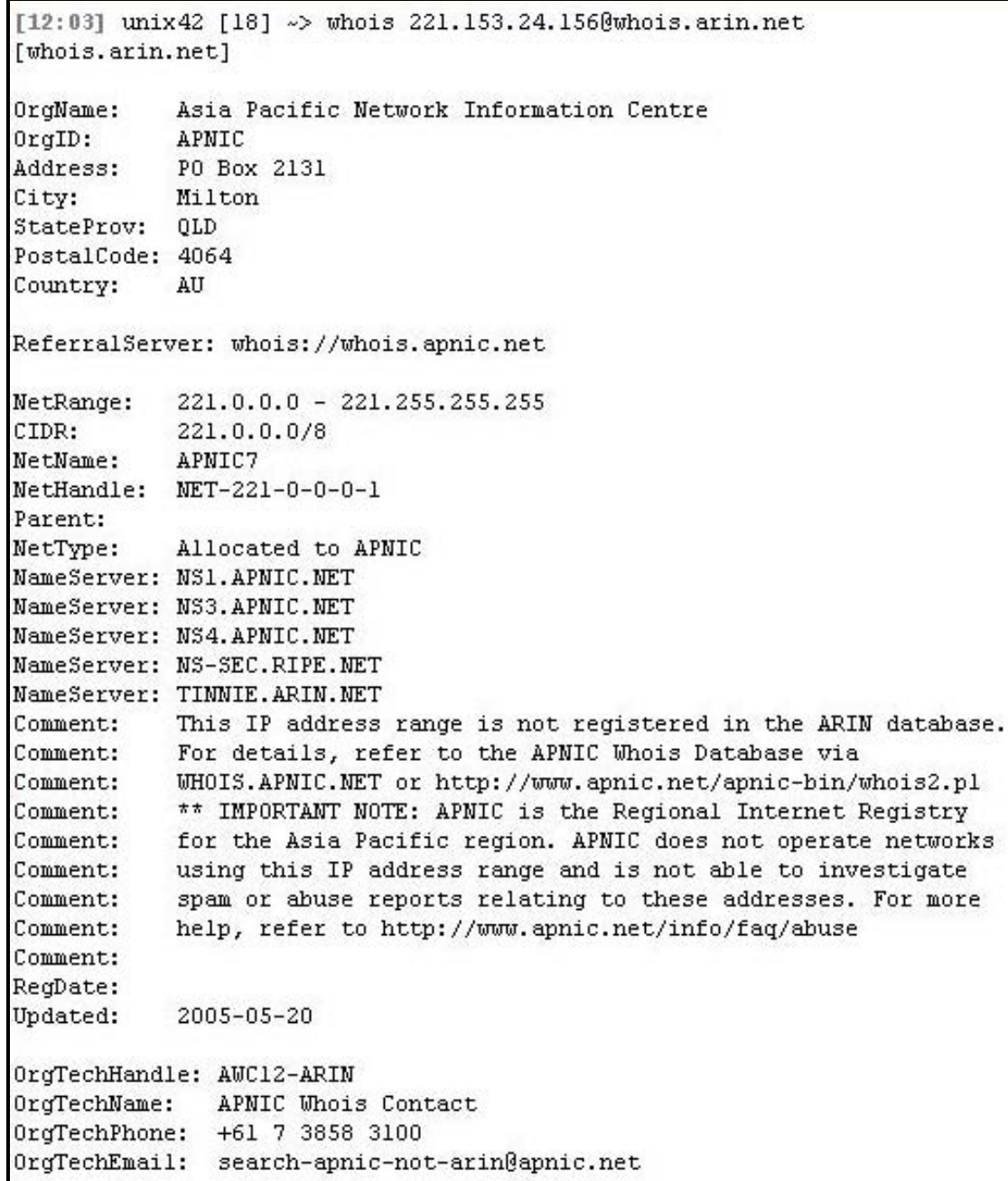

\section{Figure 78: WHOIS Query of ARIN}

Figure 78 shows that the results of the query found the IP address registered to a net block within the Asia Pacific Network Information Centre (APNIC), which is the Asian equivalent of ARIN. The results also show that APNIC has its own database (whois.apnic.net) that can be queried in order to find more specific information. As a result, the following command is used to query APNIC's database: whois 221.153.24.156@whois. apnic. net. This time the results of the query yield information about the net block owner: 


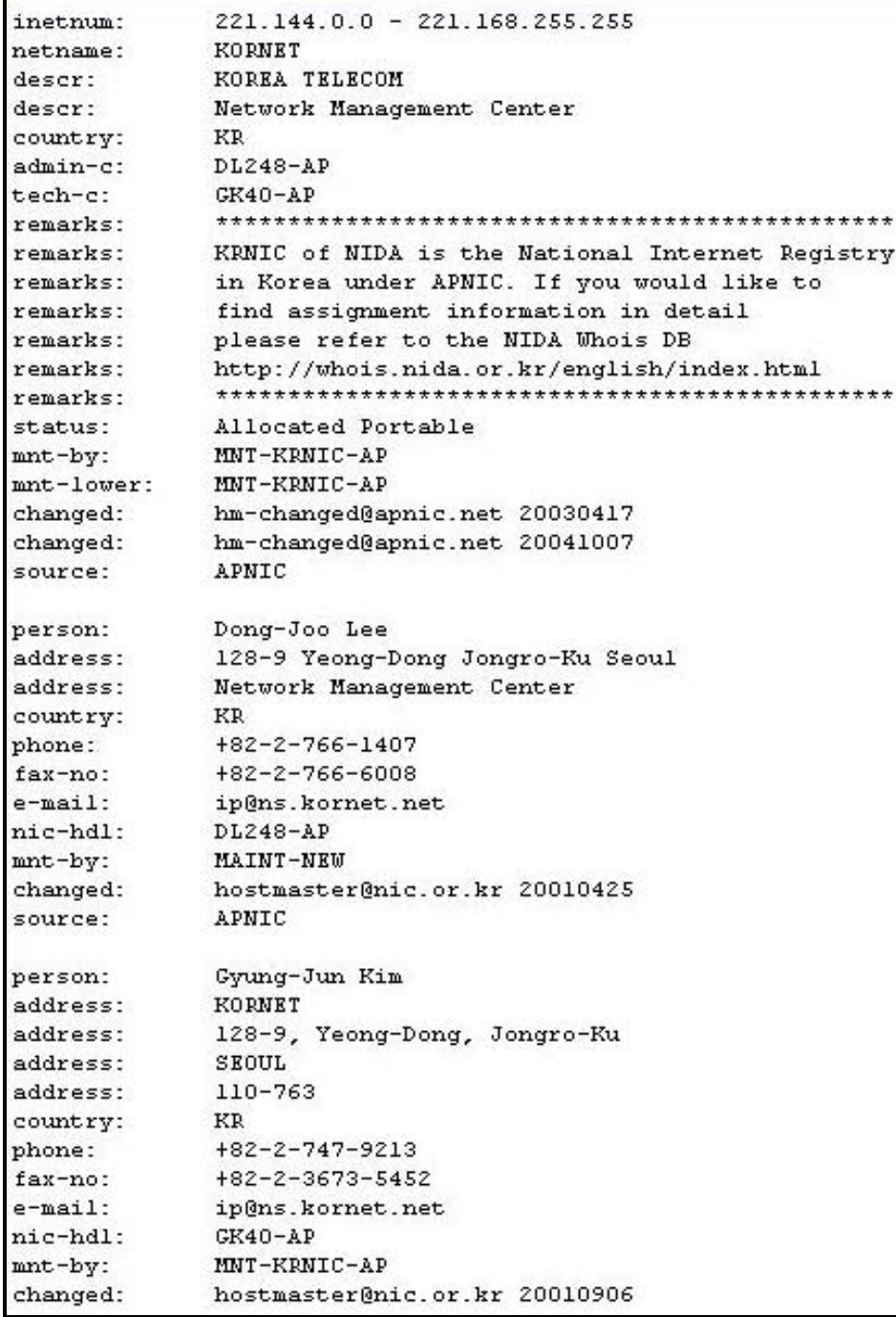

\section{Figure 79: WHOIS Query of APNIC}

Figure 79, the second whois query, shows that the IP address in question is from Korea Telecom, an ISP in Korea. From this information one can speculate that either the spoofed email came from a home user's computer that was compromised or the culprit set up a temporary account to send spoofed email; however, one cannot be certain without more information. The WHOIS information provided by APNIC also indicates a third WHOIS database, whois.nida.or.kr, which should be queried to see whether it provides even more information. At this point, if one wishes to seek out the identity of the sender, the ISP will need to be contacted. Since most home users do not have a static IP address, one address can shuffle through multiple users. Therefore, it is important to be able to determine the time the email was sent so that the IP address can be matched with the correct user account. Note that determining the user's identity may hinge on the ISP's policies, their willingness to cooperate, how long they maintain archived logs, the level of detail contained in their logs, and the accuracy of their logs. Depending on the situation and location, legal issues and implications may also need to be considered. 


\subsubsection{WHOIS Information for a Domain Name}

The most efficient method to find accurate WHOIS information for a domain name is to start from the top-level domain and work down. The following "Received" header will be used as an example:

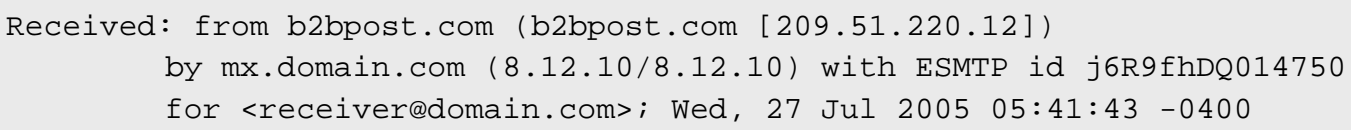

This header shows that the receiving mail server recorded the sender's IP address to be 209.51.220.12 and its domain name to be b2bpost.com, which matches the HELO information provided by the sender. A quick nslookup of the recorded IP address verifies that the email did, in fact, come from the b2bpost.com domain.

The first step to take is to find out the WHOIS server for the .com domain. This is done by querying the WHOIS database for the Internet Assigned Number Authority (IANA), which is whois.iana.org. The command for this query is whois com@whois. iana.org. Figure 80 displays the results.

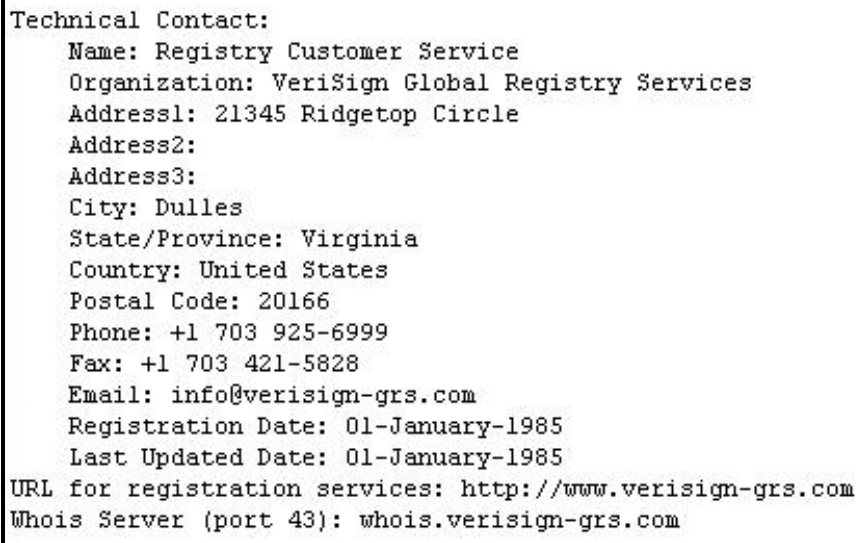

Figure 80: WHOIS Query of IANA

Among the information that is returned from the query is the WHOIS server, whois.verisigngrs.com, for the .com domain. One can be assured that querying this server will yield information regarding b2bpost.com. Sure enough, the following query yields the information shown in Figure 81: 


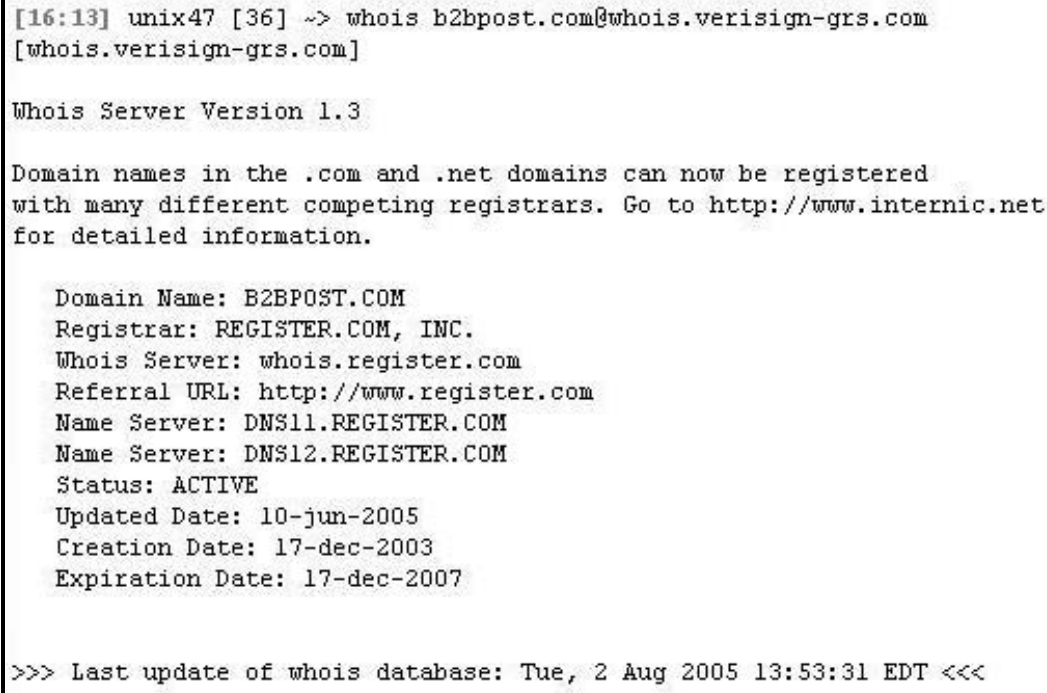

\section{Figure 81: Query of .com WHOIS Database}

This query returned some basic information regarding the domain b2bpost.com and also provided the WHOIS server, whois.register.com, of the company where b2bpost.com registered their domain. This WHOIS server should provide definitive information regarding the domain in question. The third query, whois b2bpost.com@whois.register.com, produced detailed information about the domain, as shown in Figure 82.

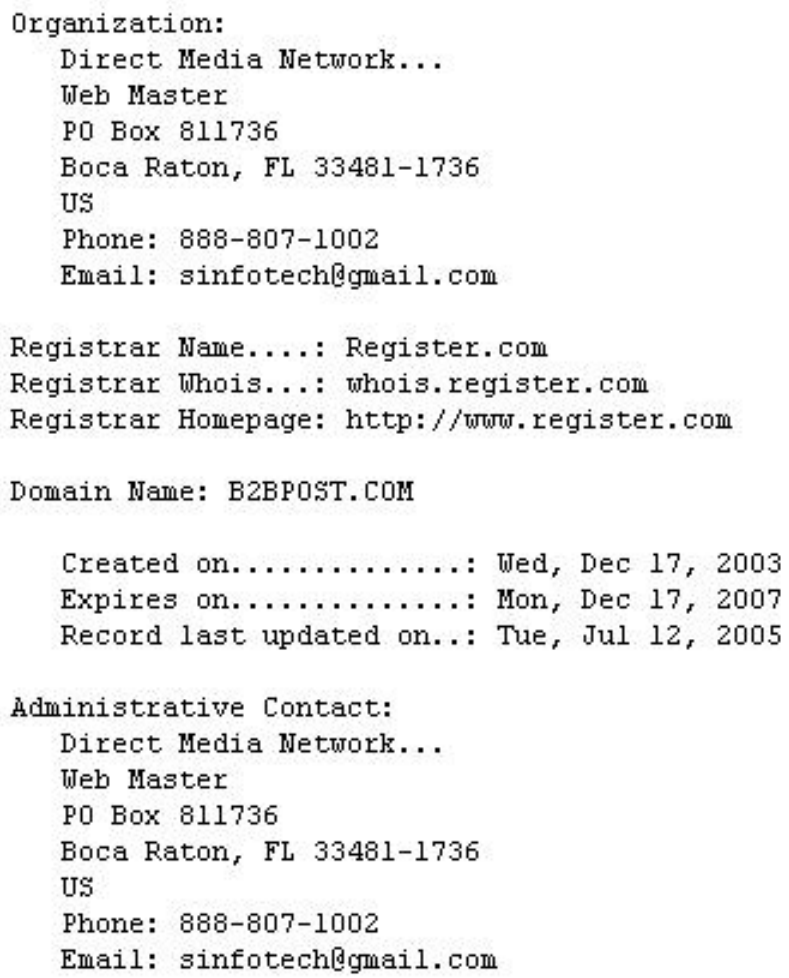

Figure 82: Query of the Registrar's WHOIS Database 
Further investigation will need to be done to determine the validity of the WHOIS information. The same issues mentioned at the end of Section 5.3.2.1 may apply.

\subsubsection{Traceroute}

While WHOIS information may yield contact information, it may not necessarily correspond to the location of the computer being investigated. It may be the case that the computer in the WHOIS database is registered to someone in a different geographical location than the actual machine. Traceroute determines the path a packet takes to a specific computer. This is done by sending Internet Control Message Protocol (ICMP) packets bound for the target computer and incrementing the time-to-live (TTL) for each packet. The first packet is sent with a TTL of 1 so that the packet dies after the first hop. The router at that hop responds and the traceroute utility now knows the first hop along the path. Traceroute continues to do this so that the second hop, third hop, etc. respond. By the time an ICMP packet reaches its destination, traceroute has mapped the entire path to it. Since the ICMP packets are sent directly from the computer that is running the traceroute, one may desire a different approach if a low-profile needs to be kept. There are many websites that allow users to perform traceroutes online. The benefit to performing a traceroute online is that the ICMP packets will originate from the web server instead of one's own machine. A good website for performing traceroutes is http://www.dnsstuff.com.

The traceroute utility can help one pinpoint the true geographic location of a system. In Windows the command to use is tracert. The following example illustrates how an organization can reside in one location and one of their servers can be in another. The SANS Institute is a computer security organization located in Bethesda, Maryland. Performing a reverse DNS lookup on their website, http://www.sans.org, reveals that the IP address of one of their web servers is 64.112.229.132. From a Windows command prompt, the following command is used to determine the location of this machine: tracert 64.112 .229.132 (Figure 83). 


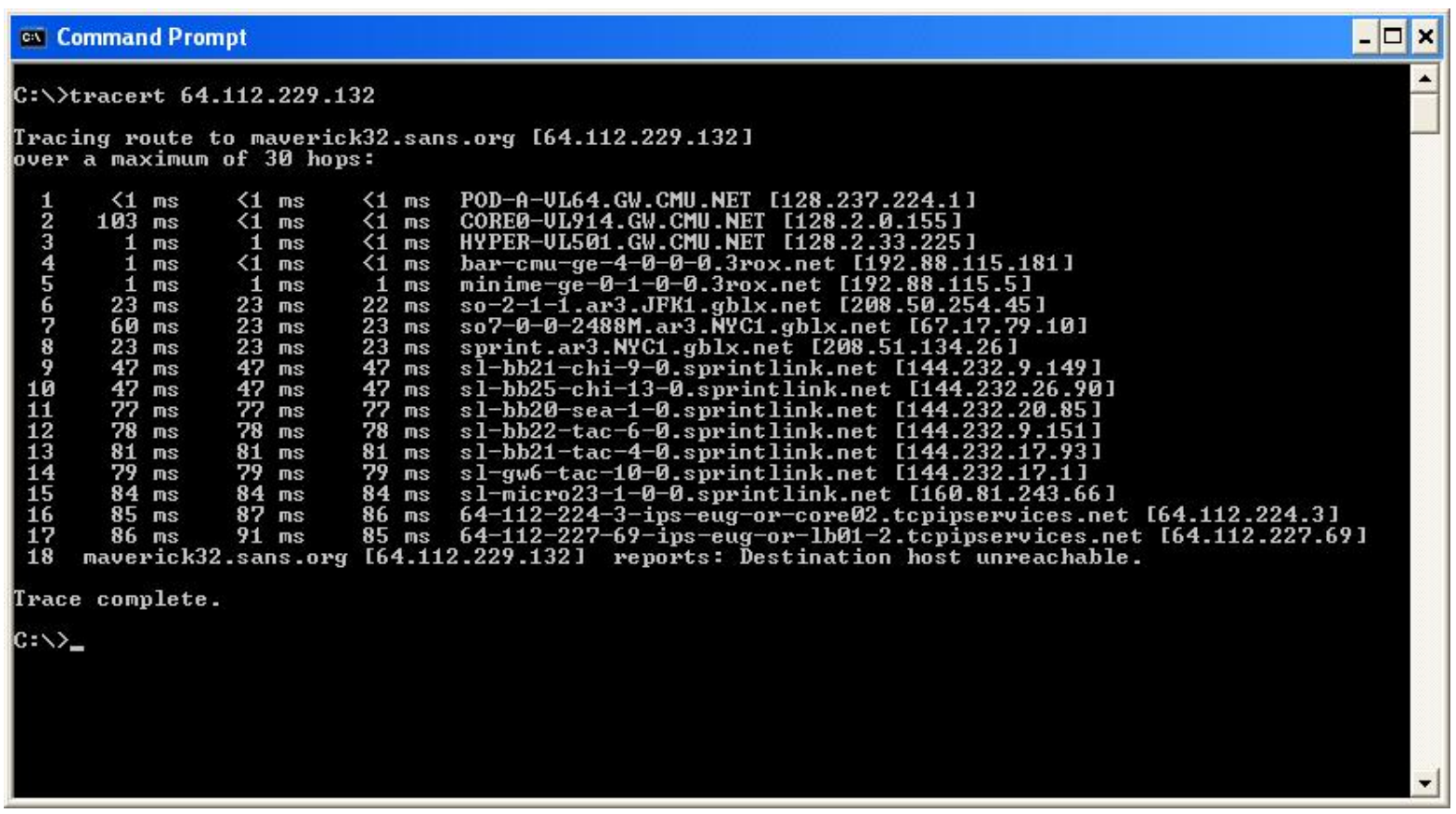

Figure 83: Traceroute Example

The final destination, 64.112.229.132, did not reply to the ICMP packet, which is typical of systems that have been hardened for security purposes. The point of interest is the hop before the final destination. Hop 17 is in the tcpipservices.net domain. A quick WHOIS query on this domain shows that the tcpipservices.net appears to be some type of service provider in Eugene, Oregon. Therefore, while the SANS Institute is located in Maryland, their web servers are in Oregon [Mandia 01].

\subsubsection{Sam Spade}

Much of the information gathered using nslookup and whois can also be collected using Sam Spade, a Windows tool that contains various network utilities such as whois, traceroute, IP block lookup, DNS check, and ping. Sam Spade is a freeware tool; it can be downloaded from the Sam Spade website at http://www.samspade.org/ssw/. Some of the utilities in Sam Spade are also available directly from the Sam Spade home page: http://www.samspade.org. Another website containing some very useful online tools is http://www.dnsstuff.com. All of the data collection techniques outlined in the previous sections can be performed using Sam Spade. 


\section{Summary}

No legitimate use for spoofed email

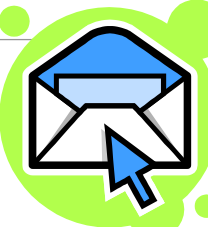

Lack of authentication in SMTP

TCP connection leaves traceable fingerprint

Spoofed email sent many different ways

Header interpretation is the key

Tracing email requires investigative work

2005 Carnegie Mellon University

CERT

\subsection{Summary}

There is no legitimate reason for spoofed email to be sent. At best, email spoofing is used by unethical advertisers who churn out billions of messages and hide their tracks to avoid the repercussions of their actions. At worst, email spoofing is used to propagate all types of malicious software and to aid in various criminal activities, the consequences of which extend beyond the digital realm. The lack of authentication in SMTP is the major contributor to the spoofed email problem currently facing society. It was designed at a time when the number of users was so few that everybody knew everyone else. However, the vast frontier of the Internet has changed the paradigm to one requiring security. While a change may not be on the horizon in the immediate future, dealing with spoofed email is not a lost cause. Because TCP is used for mail delivery, senders are not able to completely cover their tracks. The TCP handshake allows the receiving mail hosts to stamp the sender's true identity in the "Received” header. As a result, spammers have responded with a variety of techniques to muddle their tracks as much as they can. However, through email header interpretation and various tools and techniques, security professionals have the ability to trace the true origins of spoofed messages. 


\section{References}

URLs are valid as of the publication date of this document.

[Ash 95]

[Barnett 02]

[Bauer 05]

[Duda 87]

[Galleon 04]

[Haas 04]

[Lamp 78]

[LP 05]

[Lucke 04]

[Mandia 01]
Ashton, P. Algorithms for Off-Line Clock Synchronization (Technical Report TR COSC 12/952). Christchurch, New Zealand: Department of Computer Sciences, University of Canterbury, 1995.

Barnett, Ryan. Monitoring VMware Honeypots.

http://honeypots.sourceforge.net

/monitoring_vmware_honeypots.html (2002).

Bauer, Michael D. Linux Server Security, $2^{\text {nd }}$ Edition. Sebastopol, CA: O’Reilly, 2005.

Duda, A.; Harrus, G.; Haddad, Y.; Bernard, G. "Estimating Global Time in Distributed Systems.” Proceedings of the 7th International Conference on Distributed Computing Systems (ICDCS '87). Berlin, Germany, Sept. 1987. Los Alamitos, CA: IEEE Computer Society Press, 1987. http://www.informatik.uni-trier.de/ ley/db/conf/icdcs /icdcs87.html.

Galleon. GPS Time Server. http://www.ntp-time-server.com/gpstime-server/gps-time-server.htm (2004).

Haas, Juergen. Linux / Unix Command: checkconfig. http://linux.about.com/library/cmd/blcmdl8_chkconfig.htm (2004).

Lamport, L. "Time, Clocks, and the Ordering of Events in a Distributed System.” Communications of the ACM 21 (1978): 558-565.

Log Parser 2.2 Documentation. Distributed with Log Parser 2.2.

Lucke, Ken. Reading Email Headers.

http://www.stopspam.org/email/headers.html (2004).

Mandia, Kevin \& Prosise, Chris. Incident Response. Berkley, California: McGraw-Hill, 2001. 
[McWilliams 05]

[Mills 91]

[Nolan 05]

[NWG 01]

[Ristenpart 04]

[Sendmail 05]

[SourceForge 04]

[Tan 02]

[ULP 05]

[Wikipedia 05a]

[Wikipedia 05b]

[Wikipedia 05c]

[Wikipedia 05d]
McWilliams, Brian. "Hijacked by Spammers." O'Reilly Network. http://www.oreillynet.com/pub/a/network/2005/03/14 /spammerhijack.html (2005).

Mills, D. L. "Internet Time Synchronization: The Network Time Protocol." IEEE Trans. Communications 39, 10 (October 1991): 1482-1493.

Nolan, Richard; O’Sullivan, Colin; Branson, Jake; \& Waits, Cal. First Responders Guide to Computer Forensics (CMU/SEI-2005HB-001). Pittsburgh, PA: Carnegie Mellon University, Software Engineering Institute, 2005. http://www.sei.cmu.edu/publications /documents/05.reports/05hb001.html.

Network Working Group. RFC 2821. http://www.faqs.org/rfcs/rfc2821.html (2001).

Ristenpart, Thomas; Templeton, Steven; \& Bishop, Matt. "Time Synchronization of Aggregated Heterogeneous Logs." http://ultimate.cs.ucdavis.edu/SecSemApril04.ppt (2004).

Sendmail, Inc. Datasheet: Sendmail Outbound Mail Management Solution. http://www.sendmail.com/pdfs/datasheets/ds_hvms.pdf (2005).

SourceForge. Project Info - Swatch.

http://sourceforge.net/projects/swatch (2004).

Tanenbaum, Andrew S. \& van Steen, Maarten. Distributed Systems: Principles and Paradigms. Singapore: Pearson Education, 2002.

The Unofficial Log Parser Support Site. http://www.logparser.com/ (2005).

Wikipedia. American Registry for Internet Numbers. http://en.wikipedia.org/wiki/ARIN (2005).

Wikipedia. Coordinated Universal Time. http://en.wikipedia.org/wiki/UTC (2005).

Wikipedia. Global Positioning System.

http://en.wikipedia.org/wiki/Global_positioning_system (2005).

Wikipedia. Regular Expressions.

http://en.wikipedia.org/wiki/Regular_expression (2005). 
[Wikipedia 05e] Wikipedia. Simple Mail Transfer Protocol.

http://en.wikipedia.org/wiki/Simple_Mail_Transfer_Protocol (2005).

[Wikipedia 05f] Wikipedia. SQL. http://en.wikipedia.org/wiki/SQL (2005). 


\begin{tabular}{|c|c|c|c|c|}
\hline \multicolumn{3}{|c|}{ REPORT DOCUMENTATION PAGE } & \multicolumn{2}{|c|}{$\begin{array}{l}\text { Form Approved } \\
\text { OMB No. 0704-0188 }\end{array}$} \\
\hline \multicolumn{5}{|c|}{ 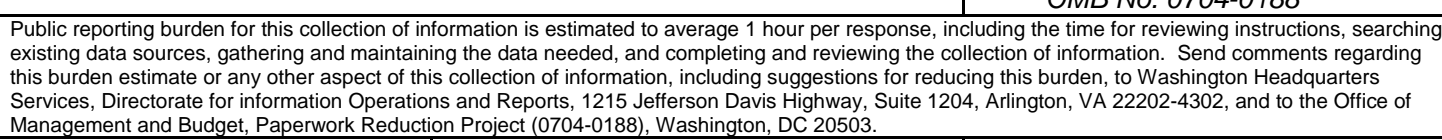 } \\
\hline AGENCY USE ONLY & \multicolumn{2}{|l|}{$\begin{array}{ll}\text { 2. REPORT DATE } \\
\text { September } 2005\end{array}$} & \multicolumn{2}{|c|}{$\begin{array}{ll}\text { 3. REPORT TYPE AND DATES COVERED } \\
\text { Final }\end{array}$} \\
\hline \multicolumn{3}{|l|}{ TITLE AND SUBTITLE } & \multicolumn{2}{|c|}{$\begin{array}{ll}\text { 5. FUNDING NUMBERS } \\
\text { FA8721-05-C-0003 }\end{array}$} \\
\hline \multicolumn{5}{|c|}{$\begin{array}{l}\text { Richard Nolan, Marie Baker, Jake Branson, Josh Hammerstein, Kris Rush, Cal Waits, Elizabeth Schweins- } \\
\text { berg }\end{array}$} \\
\hline \multicolumn{3}{|c|}{$\begin{array}{l}\text { PERFORMING ORGANIZATION NAME(S) AND ADDRESS(ES) } \\
\text { Software Engineering Institute } \\
\text { Carnegie Mellon University } \\
\text { Pittsburgh, PA } 15213\end{array}$} & \multicolumn{2}{|c|}{$\begin{array}{ll}\text { 8. } & \text { PERFORMING ORGANIZATION } \\
& \text { REPORT NUMBER } \\
& \text { CMU/SEI-2005-HB-003 }\end{array}$} \\
\hline \multicolumn{3}{|c|}{$\begin{array}{l}\text { SPONSORING/MONITORING AGENCY NAME(S) AND ADDRESS(ES) } \\
\text { HQ ESC/XPK } \\
5 \text { Eglin Street } \\
\text { Hanscom AFB, MA 01731-2116 }\end{array}$} & \multicolumn{2}{|c|}{$\begin{array}{l}\text { 10. SPONSORING/MONITORING AGENCY } \\
\text { REPORT NUMBER }\end{array}$} \\
\hline \multicolumn{5}{|l|}{ 11. SUPPLEMENTARY NOTES } \\
\hline \multicolumn{3}{|c|}{ 12A DISTRIBUTION/AVALLABILITY STATEMENT } & \multicolumn{2}{|c|}{ 12B DISTRIBUTION CODE } \\
\hline \multicolumn{5}{|c|}{$\begin{array}{l}\text { This handbook expands on the technical material presented in SEI handbook CMU/SEI-2005-HB-001, First } \\
\text { Responders Guide to Computer Forensics. While the latter presented techniques for forensically sound col- } \\
\text { lection of data and explained the fundamentals of admissibility pertaining to electronic files, this handbook } \\
\text { covers more advanced technical operations such as process characterization and spoofed email. It describes } \\
\text { advanced methodologies, tools, and procedures for applying computer forensics when performing routine log } \\
\text { file reviews, network alert verifications, and other routine interactions with systems and networks. The mate- } \\
\text { rial will help system and network professionals to safely preserve technical information related to network } \\
\text { alerts and other security issues. }\end{array}$} \\
\hline \multicolumn{3}{|c|}{$\begin{array}{l}\text { SUBJECT TERMS } \\
\text { computer forensics, information security, spoofed email, log file } \\
\text { analysis, data recovery, computer security incident }\end{array}$} & \multicolumn{2}{|c|}{$\begin{array}{l}\text { 15. NUMBER OF PAGES } \\
168\end{array}$} \\
\hline \multicolumn{5}{|l|}{ 16. PRICE CODE } \\
\hline $\begin{array}{l}\text { 17. SECURITY CLASSIFICATION } \\
\text { OF REPORT } \\
\text { Unclassified }\end{array}$ & $\begin{array}{l}\text { 18. SECURITY CLASSIFICATION OF } \\
\text { THIS PAGE } \\
\text { Unclassified }\end{array}$ & $\begin{array}{l}\text { 19. SECURITY CL/ } \\
\text { ABSTRACT } \\
\text { Unclassifie }\end{array}$ & SIFICATION OF & $\begin{array}{l}\text { 20. LIMITATION OF ABSTRACT } \\
\text { UL }\end{array}$ \\
\hline
\end{tabular}

